\title{
RESEARCH OF MODELING AND OPTIMIZATION APPROACHES FOR HYBRID EJECTOR-BASED AIR CONDITIONING CYCLE
}

WANG HAO

SCHOOL OF ELECTRICAL AND ELECTRONIC ENGINEERING 


\title{
RESEARCH OF MODELING AND OPTIMIZATION APPROACHES FOR HYBRID EJECTOR-BASED \\ AIR CONDITIONING CYCLE
}

WANG HAO

School of Electrical and Electronic Engineering

\author{
A thesis submitted to Nanyang Technological University \\ in partial fulfillment of the requirement for the degree of \\ Doctor of Philosophy
}




\section{Acknowledgments}

First and foremost, I would like to express my sincere gratitude to my supervisors, Professor Dr. Wang Youyi and Associate Professor Dr. Cai Wenjian, for their tremendous support, and invaluable guidance throughout the course of my research work.

I wish to thank my wife, Nan $\mathrm{Xi}$, for her love, understanding and encouragement. Thanks also to my friends for their presence and help to complete this significant research.

I particularly thank Dr. Wang Xinli and Dr. Yan Jia for their continuous guidance and help that I have received from them throughout the duration of my research

I also thank my colleagues and seniors in the Process Instrumentation Laboratory and the laboratory supervisor, Mr. Yock, for their help during the course of my study.

The work was funded by National Research Foundation of Singapore: NRF-CRP001-090. The other project partners are also acknowledged. 


\section{Table of Contents}

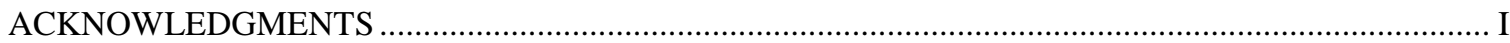

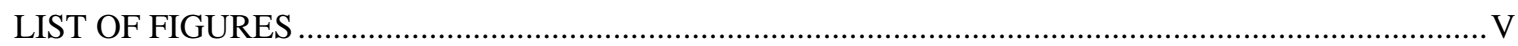

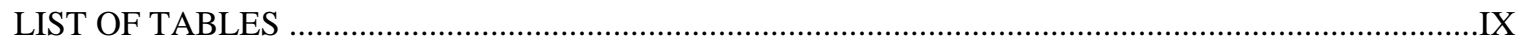

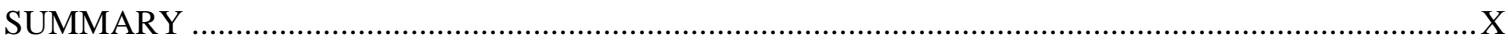

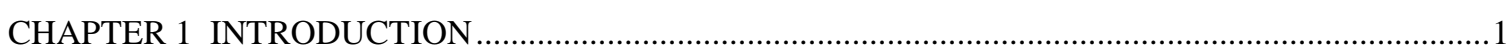

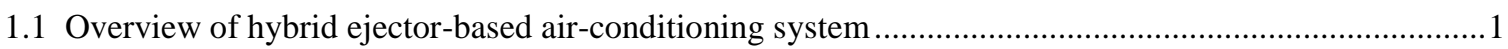

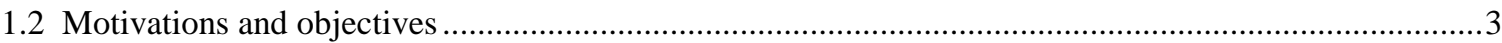

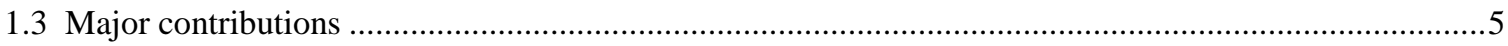

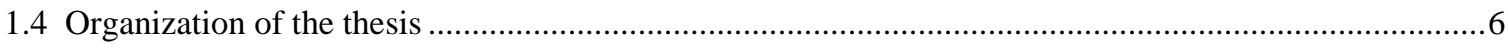

CHAPTER 2 ARTIFICIAL NEURAL NETWORK MODELING AND OPTIMIZATION METHODS......7

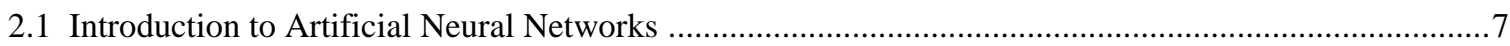

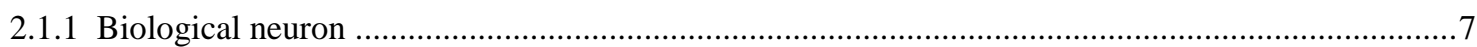

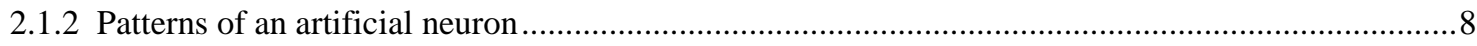

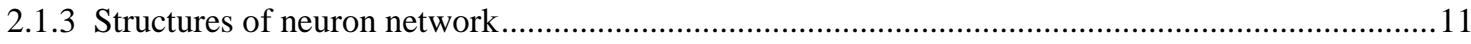

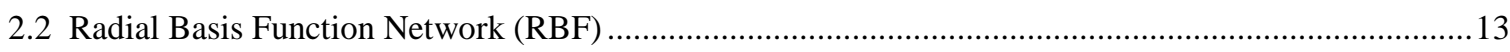

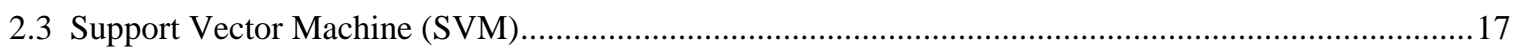

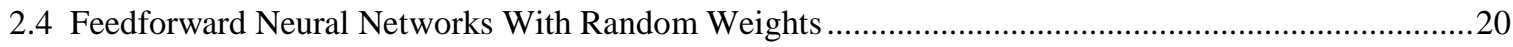

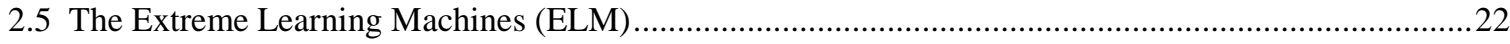

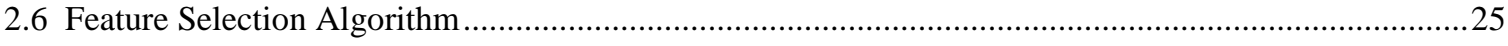

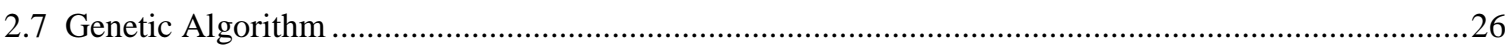

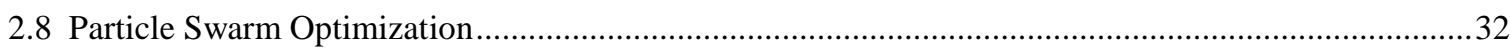

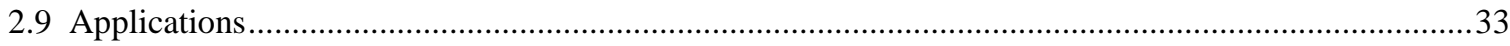

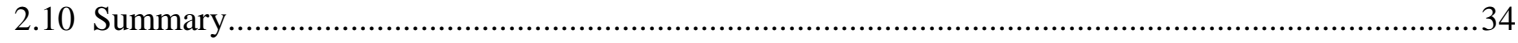

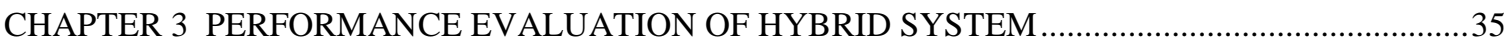

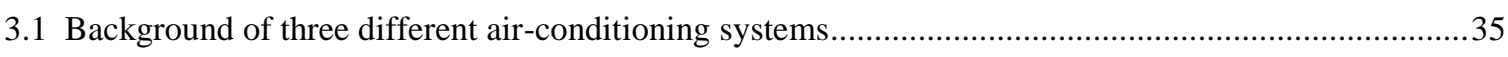

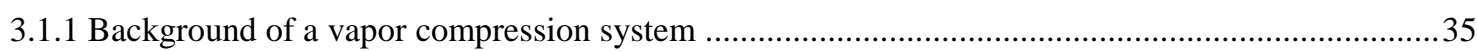

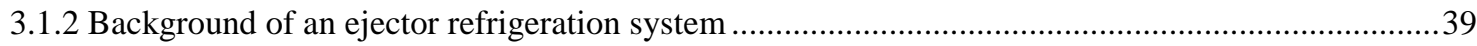

3.1.3 Background of a hybrid ejector-based refrigeration system ........................................................42

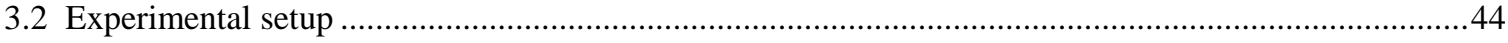

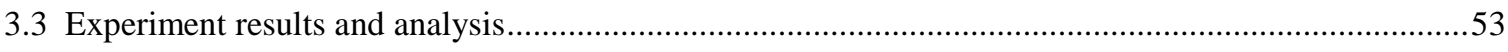

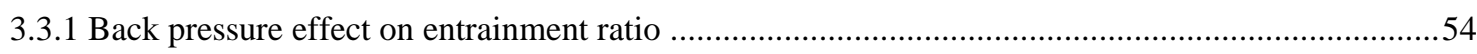

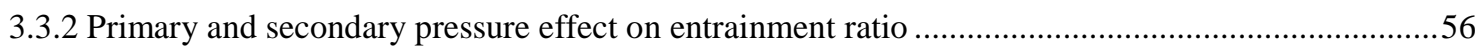

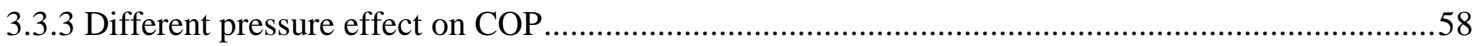




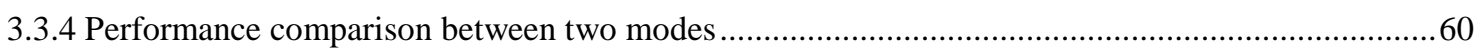

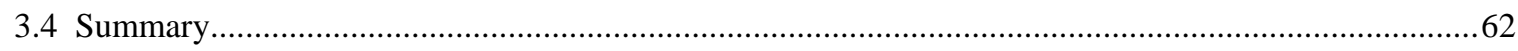

CHAPTER 4 HYBRID MODELING OF HYBRID SYSTEM..............................................................64

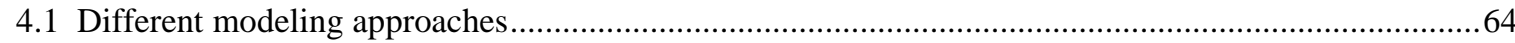

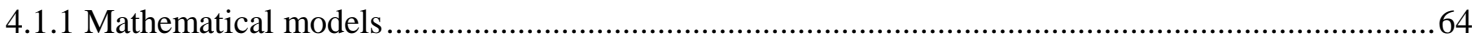

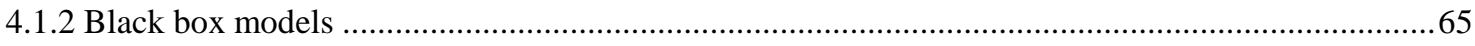

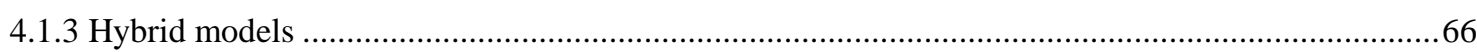

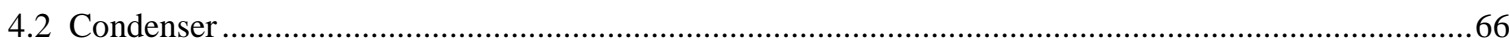

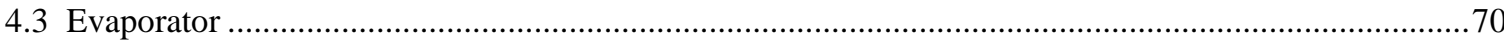

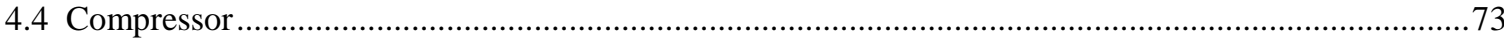

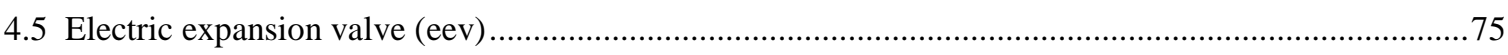

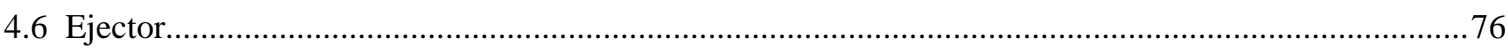

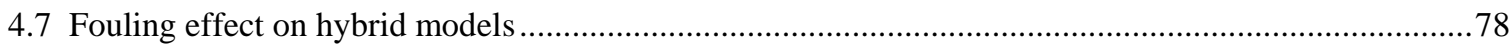

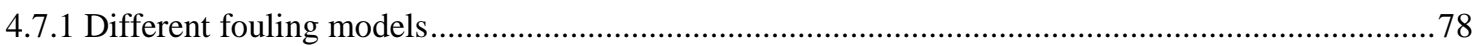

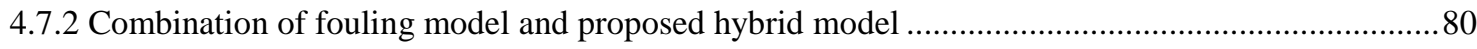

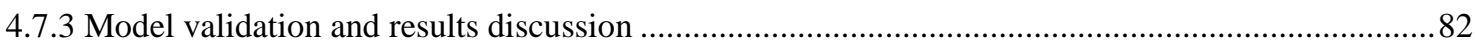

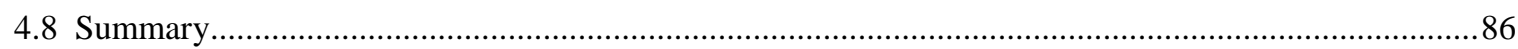

CHAPTER 5 ARTIFICIAL NEURAL NETWORK MODELING OF HYBRID SYSTEM........................87

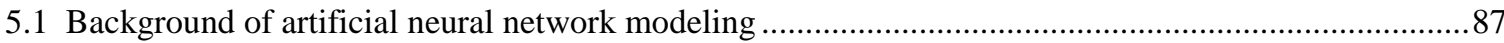

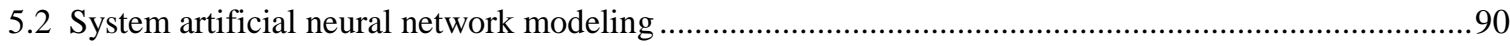

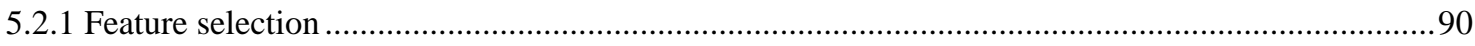

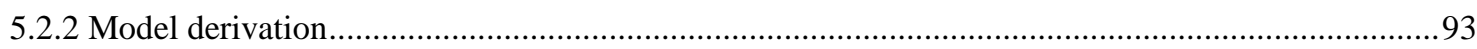

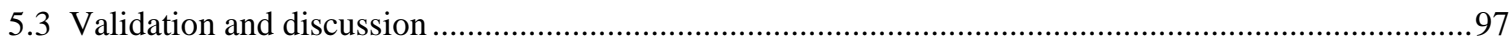

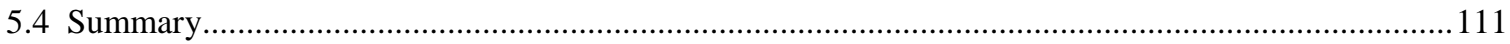

CHAPTER 6 OPTIMIZATION OF HYBRID EJECTOR-BASED AIR-CONDITIONING SYSTEM WITH ELM

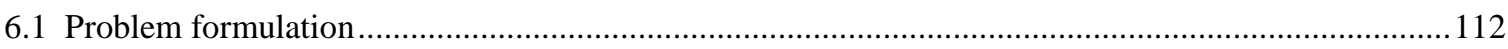

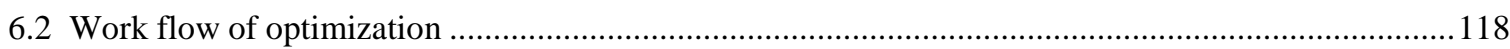

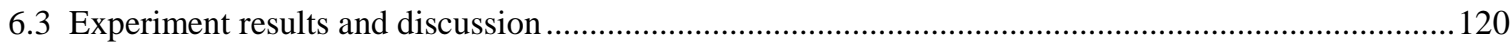

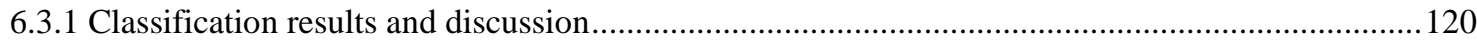

6.3.2 Total energy consumption model results and discussion ...............................................................122

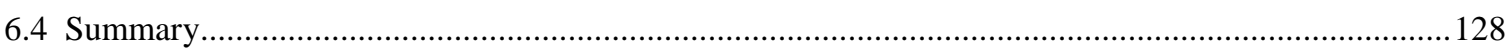

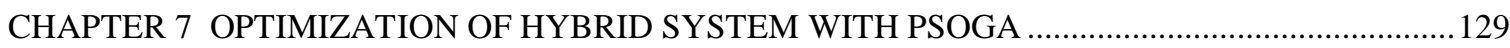

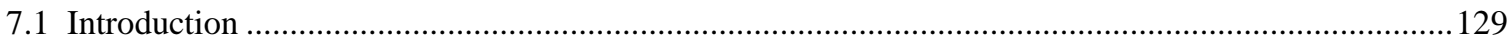

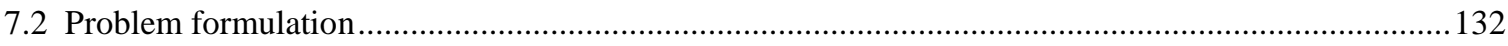

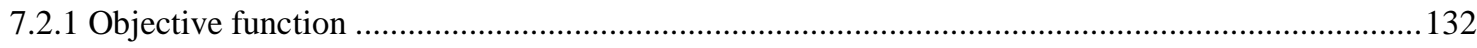

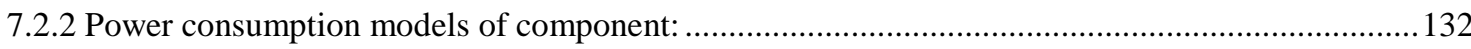




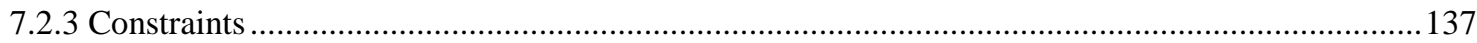

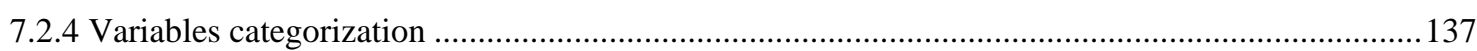

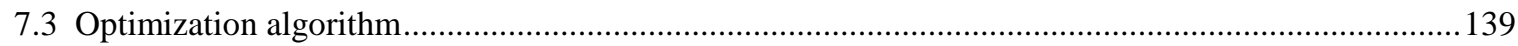

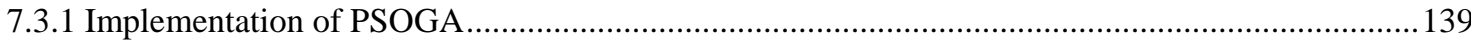

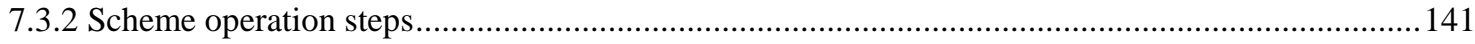

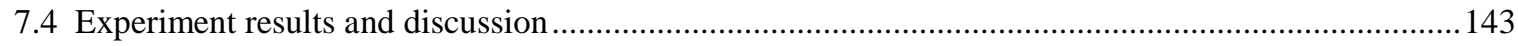

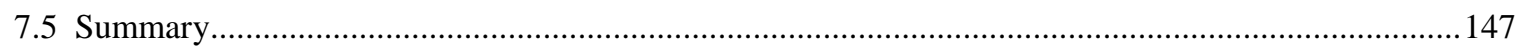

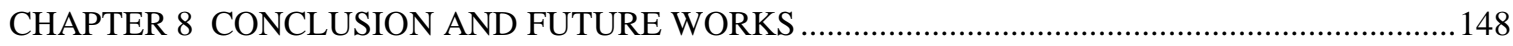

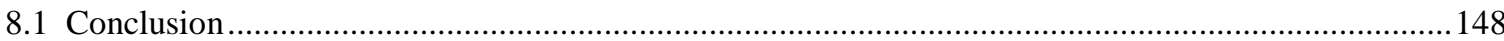

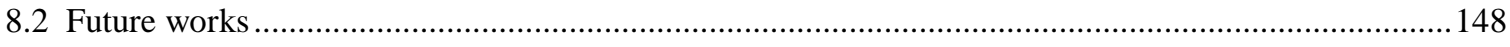

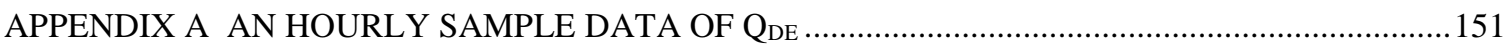

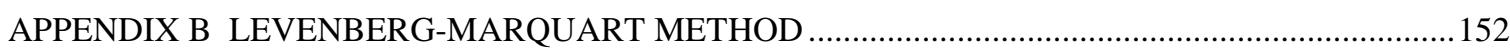

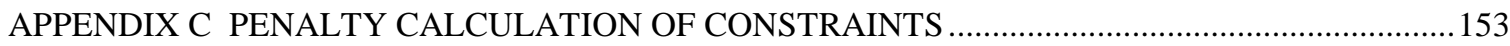

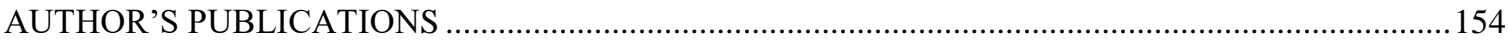

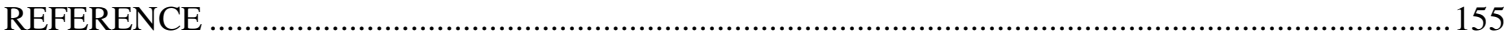




\section{List of Figures}

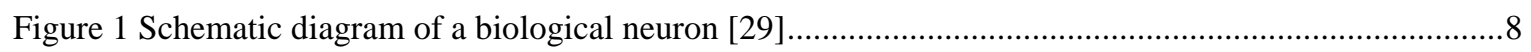

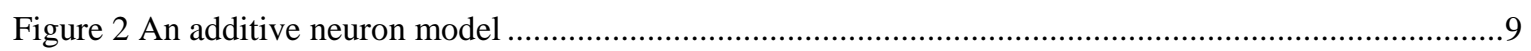

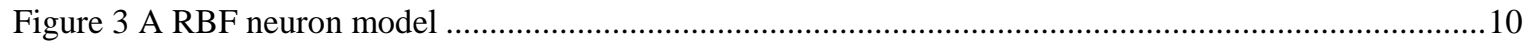

Figure 4 Structure of a Single Layer Feed-forward Neural Network ............................................................11

Figure 5 Structure of a Multi-layer Feed-forward Neural Network..............................................................12

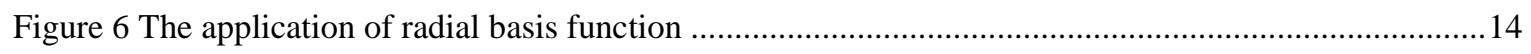

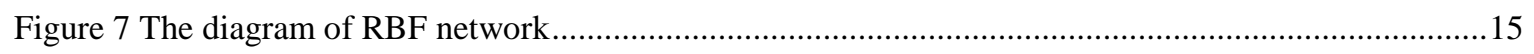

Figure 8 Structure of a feed-forward neural network with random weights .................................................21

Figure 9 Structure of a basic single hidden layer feed-forward neural network ...........................................23

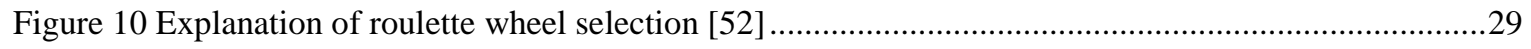

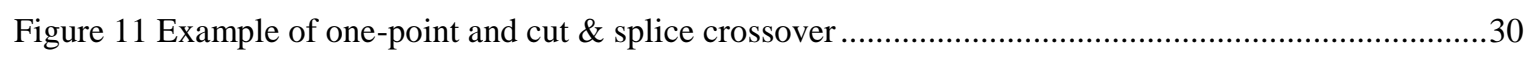

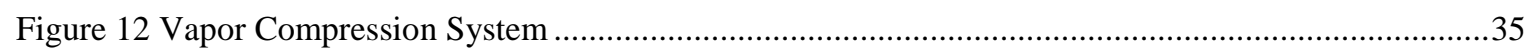

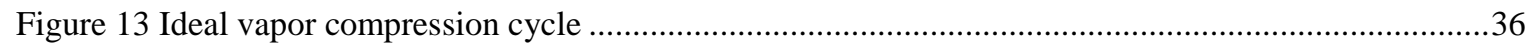

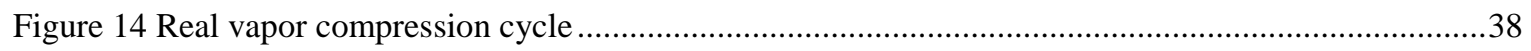

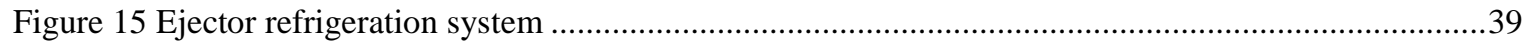

Figure 16 Schematic diagram of ejector and the relationship between pressure or velocity and location in an

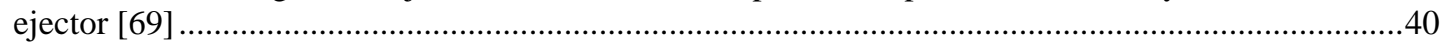

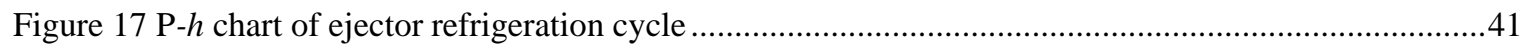

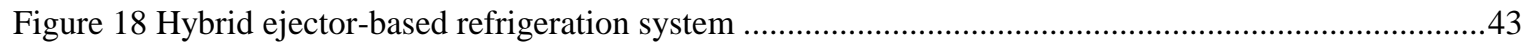

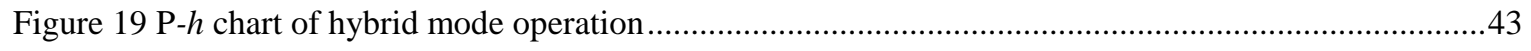

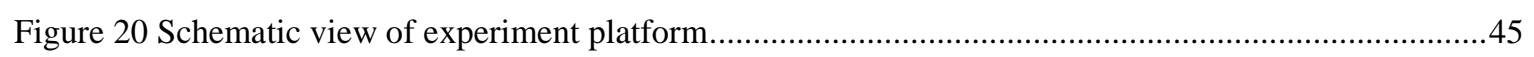

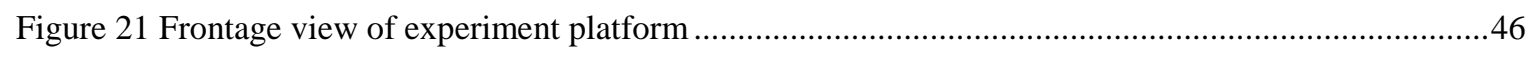

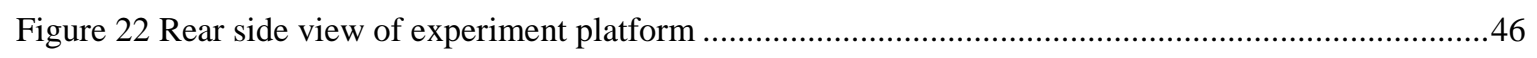

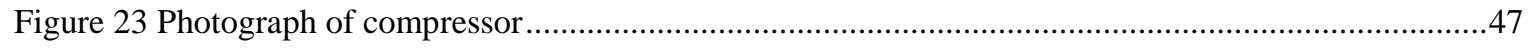

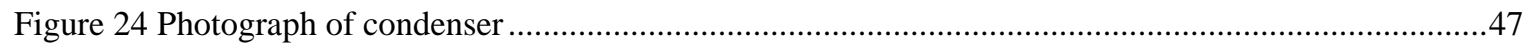

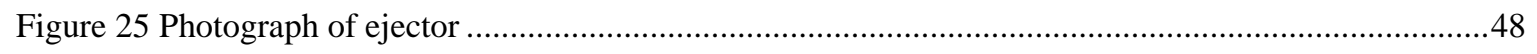

Figure 26 The detail information about ejector A ……............................................................................4

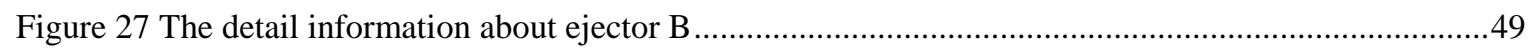

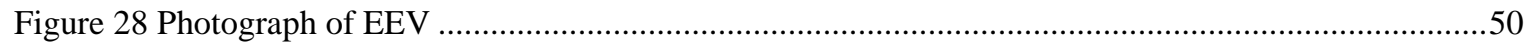

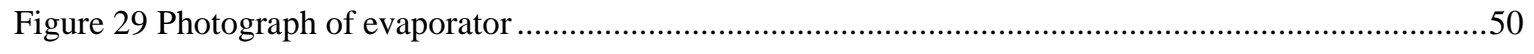

Figure 30 Photograph of generator ...................................................................................................51

Figure 31 Photograph of liquid pump .................................................................................................

Figure 32 Variation of entrainment ratio with condensing pressure under constant generating pressure........54

Figure 33 Variation of entrainment ratio with condensing pressure under constant evaporating pressure .....54

Figure 34 Variation of entrainment ratio with evaporating pressure under constant condensing pressure .....57 
Figure 35 Variation of entrainment ratio with generating pressure under constant condensing pressure.......58

Figure 36 Variation of COP with evaporating pressure under constant condensing pressure ........................59

Figure 37 Variation of COP with generating pressure under constant condensing pressure ..........................60

Figure 38 Power consumption between hybrid mode and general mode ...................................................61

Figure 39 Cooling capacity between hybrid mode and general mode ..........................................................62

Figure 40 COP between hybrid mode and general mode .........................................................................62

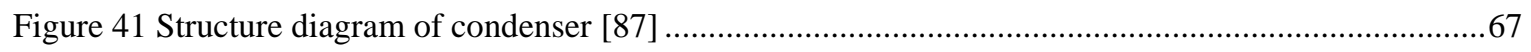

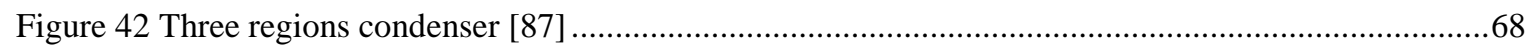

Figure 43 Experiment fitting result of condenser model ..........................................................................69

Figure 44 Relative error of condenser model ……...................................................................................69

Figure 45 Structure diagram of evaporator [86] .................................................................................... 70

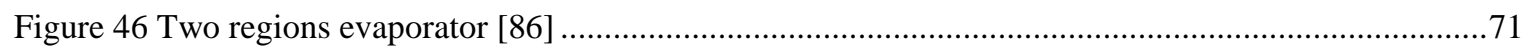

Figure 47 Experiment fitting result of evaporator model ......................................................................72

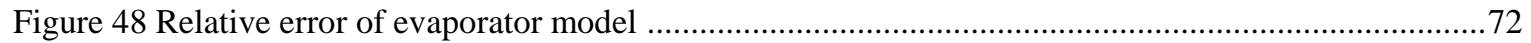

Figure 49 Experiment fitting result of compressor mass flow rate model .....................................................73

Figure 50 Relative error of compressor mass flow rate model ….............................................................74

Figure 51 Experiment fitting result of compressor power consumption model..............................................74

Figure 52 Relative error of compressor power consumption model ..............................................................75

Figure 53 Diagram of critical mode operation [89] ……........................................................................

Figure 54 Experiment fitting result of ejector model...................................................................................77

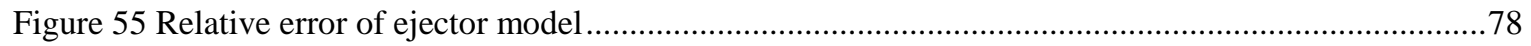

Figure 56 Fitting results of hybrid model without considering fouling condition .........................................83

Figure 57 The result of relative error of hybrid model without considering fouling condition .......................83

Figure 58 The result of measured and calculated heat transfer rate by hybrid model without considering

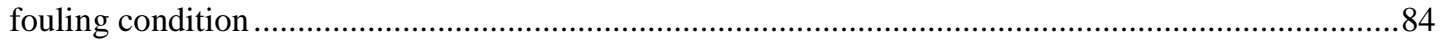

Figure 59 Fitting results of hybrid models with different orders considering fouling condition ....................84

Figure 60 Relative error of hybrid model considering fouling condition .....................................................85

Figure 61 The result of measured and calculated heat transfer rate by hybrid model considering fouling

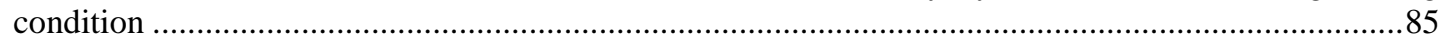

Figure 62 The SLFN of hybrid system model ………...................................................................... 93

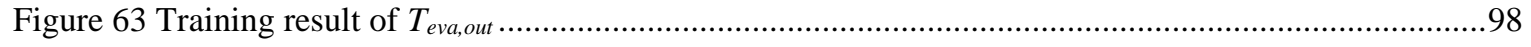

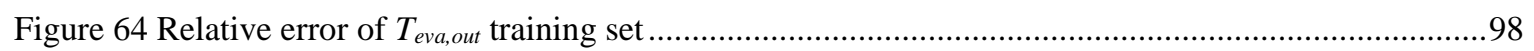

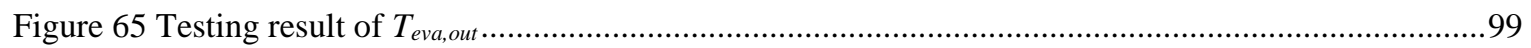

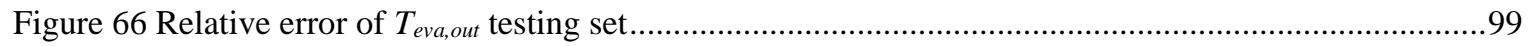

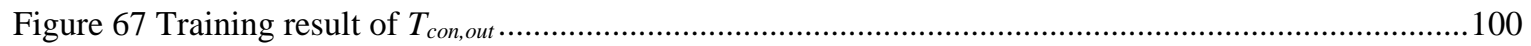

Figure 68 Relative error of $T_{\text {con,out }}$ training set ....................................................................................

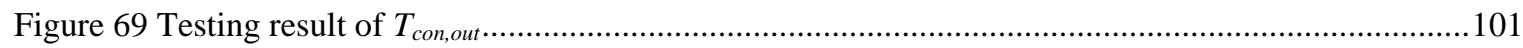

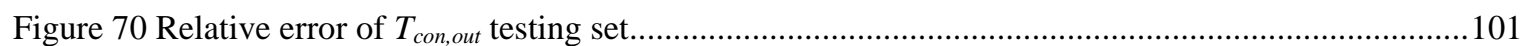




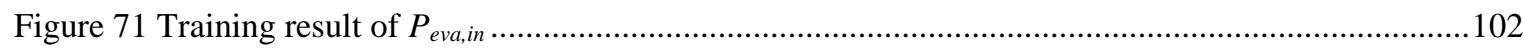

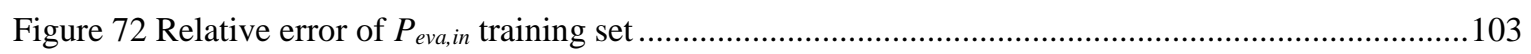

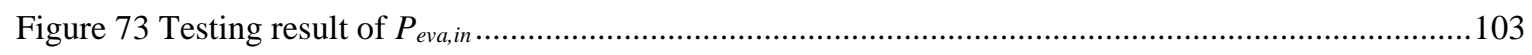

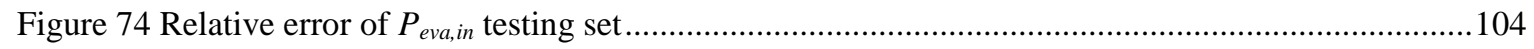

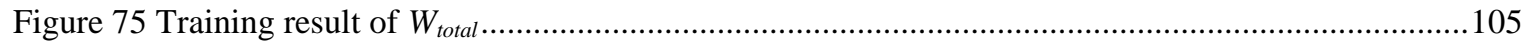

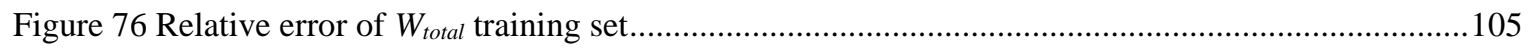

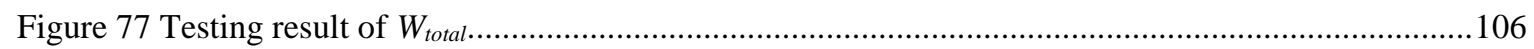

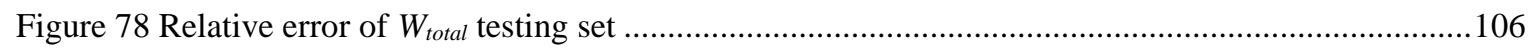

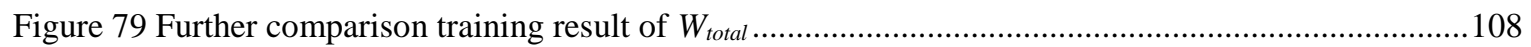

Figure 80 Further comparison relative error of $W_{\text {total }}$ training set..............................................................108

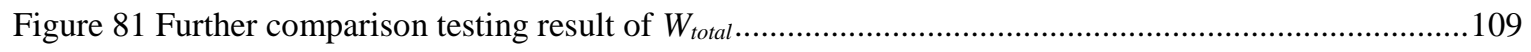

Figure 82 Further comparison relative error of $W_{\text {total }}$ testing set..............................................................109

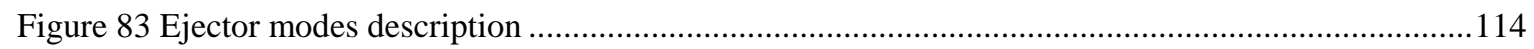

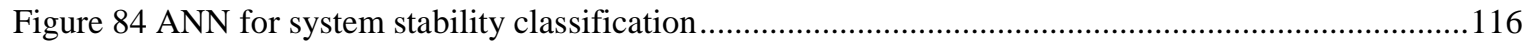

Figure 85 ANN structure of total energy consumption model.................................................................117

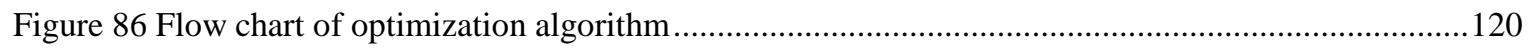

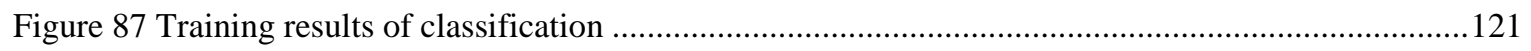

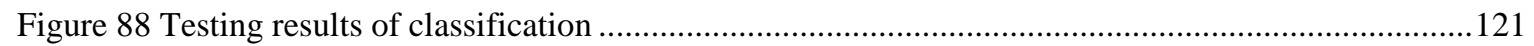

Figure 89 Fitting result of condenser fan energy consumption .............................................................123

Figure 90 Relative errors of condenser fan model .................................................................................123

Figure 91 Fitting result of evaporator fan energy consumption.............................................................124

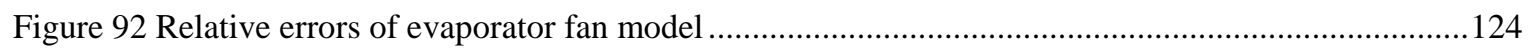

Figure 93 Fitting result of compressor energy consumption ....................................................................125

Figure 94 Relative errors of compressor model................................................................................. 125

Figure 95 Fitting result of liquid pump energy consumption ..................................................................126

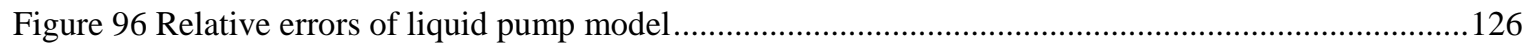

Figure 97 Comparison results of proposed optimization algorithm........................................................127

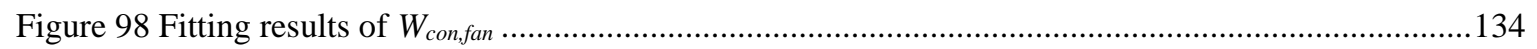

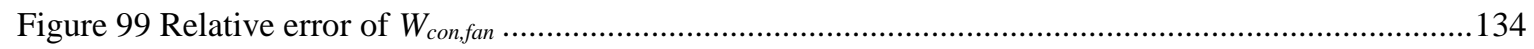

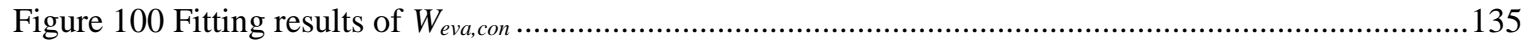

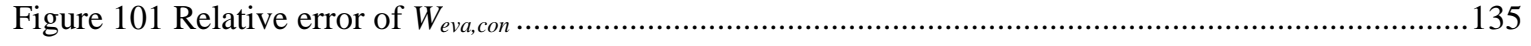

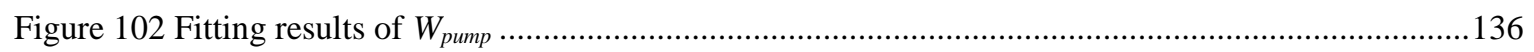

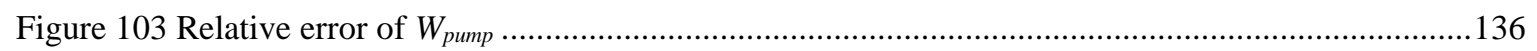

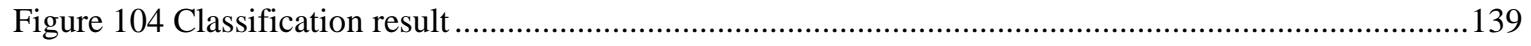

Figure 105 Flow chart of PSOGA optimization approach....................................................................142

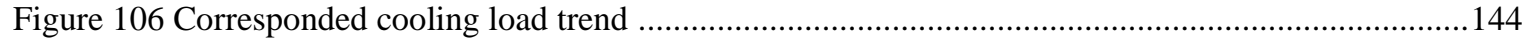

Figure 107 Energy consumptions under different optimization approach....................................................144 
Figure 108 Energy consumption and saving effect with variation of ambient temperature 146 


\section{List of Tables}

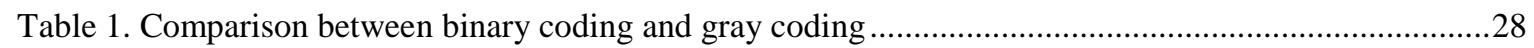

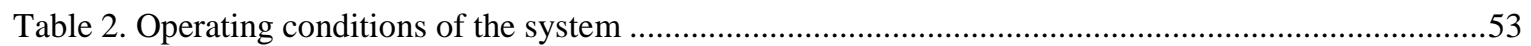

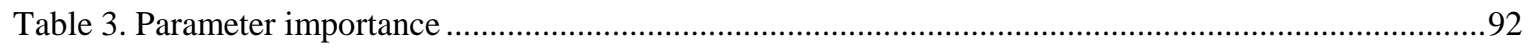

Table 4. The comparison of testing accuracies of different methods .........................................................110

Table 5. Computation of training times ...........................................................................................111

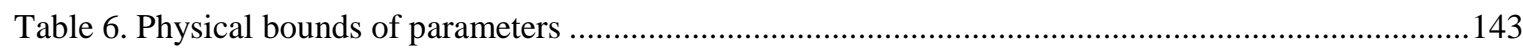

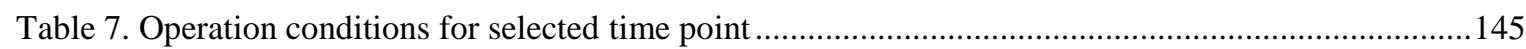

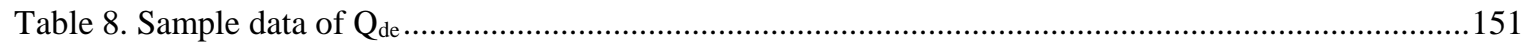




\section{Summary}

This thesis presents the research outcomes on the performance evaluation of a developed hybrid ejector-based air-conditioning system (hybrid system for short), an artificial neural network modeling for hybrid system with feature selection approaches for input parameters determination and trained with extreme learning machine (ELM), and the development and realization of a novel optimization approach based on single layer feedforward neural network (SLFN) model. Furthermore, the centralized optimization problems of hybrid system are formulated, the corresponding solution methodology is then developed. The details of these results are as follows:

1. Two ejectors with different mixing chamber diameters are applied separately for performance test and the system is operated under different working modes. The effect of generating, condensing and evaporating pressure on system performance are studied experimentally. The effect of ejector geometry parameters on ejector performance is also investigated. The performance comparison between two working modes is made and the results indicate that 1) the performance of proposed system is sensitive to the three pressures; 2) the coefficient of performance (COP) of hybrid ejector-based air-conditioning system is around 34\% higher than that of conventional compressor based system which implies a potential energy saving ability of proposed hybrid system.

2. An artificial neural network (ANN) with single hidden layer architecture is applied for modeling of hybrid system, further, an optimization issues considering the requirement of cooling capacity, ambient environment and system operating conditions, is developed. The ANN model is then combined with an exhaustive search algorithm to locate the system optimal set points under variant cooling capacities requirement. Both simulation and experiment results indicate that the ANN trained with ELM possesses lesser deviation between actual value and predicted value than the ones with other learning algorithms.

3. The model-based centralized optimization for hybrid system is developed based on evolutionary operators integrated particle swarm optimization method, which aims 
at minimizing the total energy consumption under demanded cooling capacities, with regard of components energy consumption models and corresponding physical constraints, achieving the precise estimation of the operating conditions as well as the global optimal set point.

The main contribution of this thesis is to propose two systematic approaches in optimizing the hybrid refrigeration cycle. In practice, these approaches can be adjusted accordingly to balance of performance and efficiency requirements. These approaches offer significant advantages versus traditional control and optimization methods. 


\section{Nomenclatures}

\begin{tabular}{|c|c|}
\hline $\boldsymbol{A}_{v}$ & EEV opening extent \\
\hline$c$ & coefficients \\
\hline$f$ & compressor operating frequency $(\mathrm{Hz})$ \\
\hline $\boldsymbol{K}$ & penalty functions \\
\hline $\boldsymbol{h}$ & refrigerant enthalpy $(\mathrm{kJ} / \mathrm{kg})$ \\
\hline $\boldsymbol{h}_{\text {con,vl }}$ & condenser refrigerant enthalpy difference between vapor and liquid state $(\mathrm{kJ} / \mathrm{kg})$ \\
\hline $\boldsymbol{h}_{\text {con,r,in }}$ & refrigerant enthalpy at condenser inlet $(\mathrm{kJ} / \mathrm{kg})$ \\
\hline $\boldsymbol{h}_{\text {con,r,out }}$ & refrigerant enthalpy at condenser outlet $(\mathrm{kJ} / \mathrm{kg})$ \\
\hline $\boldsymbol{h}_{\text {com,r,in }}$ & refrigerant enthalpy at compressor inlet $(\mathrm{kJ} / \mathrm{kg})$ \\
\hline $\boldsymbol{h}_{\text {com,r,out }}$ & refrigerant enthalpy at compressor outlet $(\mathrm{kJ} / \mathrm{kg})$ \\
\hline $\boldsymbol{h}_{\text {eva,sat }}$ & enthalpy of refrigerant of saturated state in evaporator $(\mathrm{kJ} / \mathrm{kg})$ \\
\hline $\boldsymbol{h}_{\text {eva,r,in }}$ & refrigerant enthalpy at evaporator inlet $(\mathrm{kJ} / \mathrm{kg})$ \\
\hline $\boldsymbol{h}_{\text {eva,r,out }}$ & refrigerant enthalpy at evaporator outlet $(\mathrm{kJ} / \mathrm{kg})$ \\
\hline $\boldsymbol{h}_{i s e n}$ & refrigerant enthalpy at compressor outlet under isentropic condition $(\mathrm{kJ} / \mathrm{kg})$ \\
\hline$\dot{\boldsymbol{m}}_{\text {con,air }}$ & air flow rate of condenser $(\mathrm{kg} / \mathrm{s})$ \\
\hline$\dot{\boldsymbol{m}}_{\text {con,air,max }}$ & maximum allowed condenser air flow rate $(\mathrm{kg} / \mathrm{s})$ \\
\hline$\dot{\boldsymbol{m}}_{\text {con,air,min }}$ & minimum allowed condenser air flow rate $(\mathrm{kg} / \mathrm{s})$ \\
\hline$\dot{\boldsymbol{m}}_{\text {eva,air }}$ & air flow rate of evaporator $(\mathrm{kg} / \mathrm{s})$ \\
\hline$\dot{\boldsymbol{m}}_{\text {eva,air,max }}$ & maximum allowed evaporator air flow rate $(\mathrm{kg} / \mathrm{s})$ \\
\hline$\dot{\boldsymbol{m}}_{\text {eva,air,min }}$ & minimum allowed evaporator air flow rate $(\mathrm{kg} / \mathrm{s})$ \\
\hline$\dot{\boldsymbol{m}}_{s}$ & ejector secondary flow rate $(\mathrm{kg} / \mathrm{s})$ \\
\hline$\dot{\boldsymbol{m}}_{p}$ & ejector primary flow rate (kg/s) \\
\hline$\dot{\boldsymbol{m}}_{r}$ & total refrigerant mass flow rate $(\mathrm{kg} / \mathrm{s})$ \\
\hline$\dot{\boldsymbol{m}}_{r, \max }$ & maximal refrigerant mass flow rate $(\mathrm{kg} / \mathrm{s})$ \\
\hline$\dot{\boldsymbol{m}}_{r, \min }$ & minimal refrigerant mass flow rate $(\mathrm{kg} / \mathrm{s})$ \\
\hline
\end{tabular}




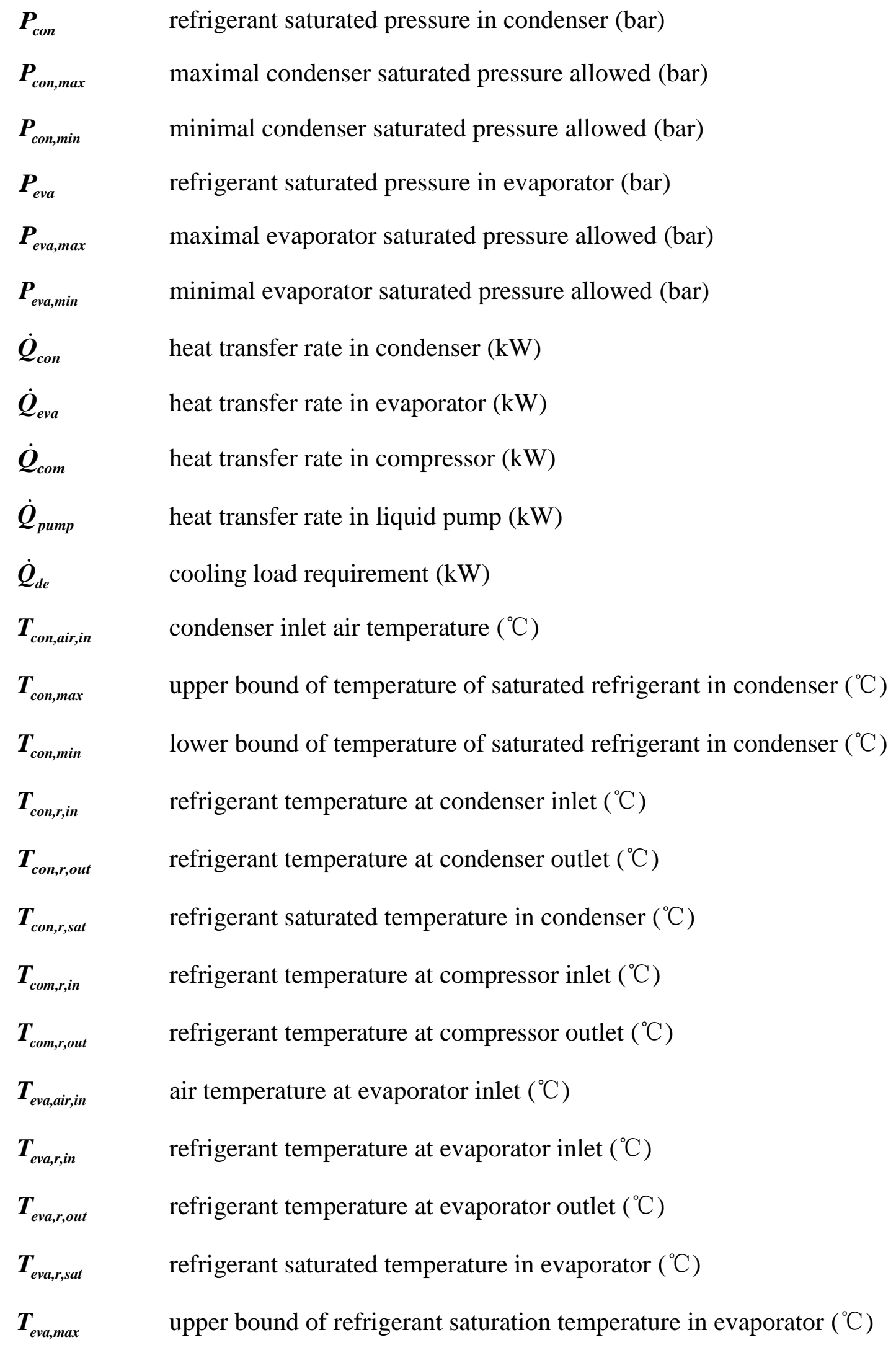


$\boldsymbol{T}_{\text {eva,min }} \quad$ lower bound of refrigerant saturation temperature in evaporator $\left({ }^{\circ} \mathrm{C}\right)$

$\dot{W}_{c o n f a n} \quad$ power consumption of condenser fan $(\mathrm{kW})$

$\dot{\boldsymbol{W}}_{\text {com }} \quad$ power consumption of compressor $(\mathrm{kW})$

$\dot{\boldsymbol{W}}_{\text {eva fan }} \quad$ power consumption of evaporator fan $(\mathrm{kW})$

$\dot{W}_{\text {pump }} \quad$ power consumption of liquid pump (kW)

$\dot{W}_{\text {total }} \quad$ total power $(\mathrm{kW})$

$\boldsymbol{x} \quad$ state vector of subsystem

$\boldsymbol{\eta}_{\text {com }} \quad$ enthalpy delivery efficiency of compressor

$\boldsymbol{\rho} \quad$ inlet refrigerant density $\left(\mathrm{kg} / \mathrm{m}^{3}\right)$

$\boldsymbol{\beta} \quad$ coefficients of energy consumption terms

$\gamma \quad$ updating coefficient of $\beta$

Subscripts

air air

con condenser

com compressor

eva evaporator

fan fan

in inlet

out outlet

$\boldsymbol{k} \quad$ number of current cycle

m mass flow rate

p primary

$s \quad$ secondary

$r \quad$ refrigerant

$\boldsymbol{\eta} \quad$ enthalpy delivery efficiency 


\section{Chapter 1 Introduction}

\subsection{Overview of hybrid ejector-based air-conditioning system}

Although ejector has been invented for decades which is earlier than compressor, the progress of ejector based refrigeration system is slow compared with vapor compression system. The reason for this is that the efficiency of vapor compression system is much higher than that of ejector refrigeration system [1]-[5]. However, due to the deteriorating environment caused by massive $\mathrm{CO}_{2}$ emission and declining global storage of fossil fuel resources, ejector refrigeration system have regained research interests. The distinctive characteristic of ejector refrigeration system is its ability to utilize low grade heat energy which can be easily acquired from automobile exhaust gas, solar radiation, industrial process and geothermal energy [6]-[9]. Moreover, because of the application of ejector, the system possesses many advantages, such as passive device (no moving components), durable life span, little maintenance cost and high reliability. The main restriction of prevalence of ejector refrigeration system is its relative low efficiency compared to vapor compression system.

To have insightful comprehension on the effect of ejector in the whole system and to improve the efficiency of ejector refrigeration system, many studies have been conducted including performance evaluation towards variety of systems. [10] investigated the effect of nozzle size, axial nozzle location, high-temperature evaporator temperature and refrigeration temperature by conducting experiment of an ejector refrigeration system. The author found that an optimum nozzle location exists and could produce a maximum coefficient of performance (COP). The results showed that ejector refrigeration system can achieve a COP of 1.7 which demonstrated a very promising future. [11] conducted experiments with an integrated ejector cooling system to analyze the system performance under design conditions. The results indicated that $9.1 \%$ COP improvement can be achieved using R22. The author then compared the system performance with the variations of 5 important parameters. The comparison illustrated that the refrigerant with high generator discharge temperature can improve the COP of the ejector cycle effectively. [12, 13] studied the effects of ejector area ratio on system performance. An ejector with replaceable nozzle was applied in order to obtain the optimum area ratios 
under operation conditions. The consequence showed that the optimum area ratios fall in the range from 3.69 to 4.76 which are smaller than other previous studies. Also the operating conditions had great impact on the influence of area ratio. [14] investigated experimentally the influence of different temperatures on ejector refrigeration system performance using R123 and R141b. The results indicated that the condenser temperature had more influence than the generator and evaporator temperature on the area ratio and the entrainment ratio in the ejector. Additionally, the author concluded that area ratios need to keep up the pace with the variation of entrainment ratio as operating conditions are changed. Thus, a variable-geometry ejector seemed to be a very promising alternative to guarantee the optimum conditions of ejector refrigeration system.

The refrigerant used in the system is also of concerned. [15] conducted a theoretical study on the evaluation of environment-friendly refrigerants with similar normal boiling points in ejector refrigeration cycle. Totally eight different kinds of refrigerants were studied comparatively. Entrainment ratios of ejector, system COP, pump power et al. of refrigerants were recorded and analyzed. The result shows that R134a and R152a have similar normal boiling points and give better performance and their COP were stable under different operation status. [16] investigated the performance of heat driven ejector refrigeration system using refrigerant R134a, R600 and R601. The effect of generator, evaporator and condenser temperature over the entrainment ratio and COP had been studied under typical operating conditions of low grade energy sources. From the result obtained, it can be observed that the working fluids had a great impact on the ejector performance and each refrigerant had its own range of operating conditions. The optimum generating temperature for R134a is lower than others and is suitable for generator temperature between 70 and $100^{\circ} \mathrm{C}$. Besides, R134a has several advantages over other refrigerants: 0 ozone depletion potential and relative lower global warming impact. Thus, $\mathrm{R} 134 \mathrm{a}$ is chosen as the working fluid in our system.

In other studies, [17] presented a novel ejector expansion refrigeration cycle which applied two evaporators using ejector as a throttling valve. The constant area mixing model was applied to perform thermodynamic analysis of the new system. Both R134a and its potential substitute R1234yf was applied for investigation. It was found that the novel system had a significant improvement in COP for both R134a and R1234yf, 
moreover, the COP improvement of the new system using R134a was higher than that of R1234yf and was able to achieve more than $17 \%$ increase at certain condensing temperature. [18] proposed an experimental investigation of the adjustable ejector in a multi-evaporator refrigeration system. Two parameters namely pressure recovery ratio (PRR) and relative pressure recovery ratio (RPRR) are measured to evaluate the system performance. The results show that the proposed system can be a promising approach to save energy significantly. Moreover, the decreasing of the primary cooling load will lead to the reduction of both PRR and RPRR. [19] explored the extent of COP improvement of a novel vapor-compression refrigeration cycle with mechanical sub-cooling using an ejector. The author developed a mathematical model to predict the performance of the cycle by using R404A and R290. The results suggest that the ejector sub-cooled cycle is better than that of the conventional cycle. The novel cycle would achieve volumetric refrigeration capacity improvement of $11.7 \%$ with $\mathrm{R} 404 \mathrm{~A}$ and $7.2 \%$ with R290. Furthermore, the COP improvement of novel cycle was $9.5 \%$ with R404A and $7.0 \%$ with R290. In addition, the improvement of COP and cooling capacity majorly depended on the operation conditions of the ejector. [20,21] which was capable of making use of waste energy from vapor refrigerant under superheated state. From the result they obtain, 15.4\% COP improvement can be realized averagely while the maximum can reach to $24.5 \%$. [22] applied an ejector for expansion purpose instead of an expansion valve for expansion work recovery in a vapor compression cycle. The author experimentally investigated the COP values for both conventional and ejector systems under same external conditions. The data obtained demonstrated that the system with ejector as the expander had better performance than the conventional one and the COP improvement was $6.2 \%-14.5 \%$ within an error of approximately 10\%. Currently, no literature has been found on attempting to connect ejector and evaporator with compressor directly.

To fill this blank, we have proposed a hybrid system in which the evaporator outlet is connected directly to ejector secondary inlet via a variable speed compressor.

\subsection{Motivations and objectives}

Singapore has a tropical rainforest climate with that is always hot and humid with lot of rains. The average annual ambient temperature during day time is around $30^{\circ} \mathrm{C}$ while night time temperature drops below $23^{\circ} \mathrm{C}$ and an average annual ambient relative humidity 
of $85 \%$ [23]. Therefore, the heat ventilation and air-conditioning system (HVAC) is essential to people living in Singapore. Although people enjoy the pleasant environment generated by HVAC, the electricity consumption in building HVAC systems is very high. According to BCA's Green Mark program, more than half of the total electric energy of the country is used for operating air conditioning systems. Additional, data obtained via survey indicates that the energy consumption of a middle size commercial building can even reach about $1,000,000 \mathrm{kWh}$ per month. Considering that the current electricity bill in Singapore is around 23 cents per $\mathrm{kWh}$, the energy cost solely for air conditioning is about 230,000 SGD. It is estimated that, in Singapore, there are more than 10,000 large commercial and industrial buildings that are equipped with central air conditioning systems [24]. It is beyond imagination of how much money will cost to run these systems. With the aim to utilize low-grade heat energy as well as reduce energy bills, the passive device, ejector, is integrated into a conventional vapor compression system. Although the hybrid system is different from vapor compression system, the research results of control and optimization methodologies towards vapor compression system are helpful for thorough investigation of hybrid system. Currently, the conventional vapor compression system is controlled in terms of superheat, which is to be maintained at its minimal tolerable degree in order to increase system performance. The shortcoming of this strategy is that the energy saving potential of all components is neglected and the determination of such minimal tolerable degree as well as the control scheme of compressor and expansion valve highly relies on user's experience. It would be more difficult to handle with hybrid system since more components are incorporated. Some classical methods for air conditioning system optimization based on gradient learning algorithm are proposed, however, since states of hybrid system can be severely interacted, these methods usually converge to a local minimum rather than the desired global optimal solution. System effective optimization techniques for HVAC based on intelligent algorithm have been proposed, but these researches are focused on the whole system, the vapor compression cycle within it is simply represented by a polynomial function, the interactions between major components inside the cycle are neglected. Therefore, the optimal designs of hybrid system is essential for reducing the energy consumption, and the optimization methodologies developed in this thesis for hybrid system will significantly advance the 
research and manufacturing in the area of refrigeration and air-conditioning systems in the near future.

\subsection{Major contributions}

The major contributions in this study include

- Performance evaluation of proposed hybrid system under different operation conditions: such as two ejectors with different geometric parameters, ejector entrainment ratio with variation of different pressures and COP comparison between different operation modes. This segment will be illustrated and discussed thoroughly in Chapter 3 and the related work is published in [77];

- Modification of component heat exchanger hybrid models considering fouling effect is presented, and then the comparisons of proposed fouling model with different orders of polynomial functions as well as model performance before and after modification are presented. This segment will be shown in Chapter 4;

- Development of the ANN model trained with the extreme learning machine, the model is also trained with $\mathrm{BP}, \mathrm{RBF}$, feedforward with random weights and SVM to help indicate the better performance of extreme learning machine. This segment will be presented in Chapter 5 for further investigation and the related work is published in [110];

- Formulation of the centralized optimization methodology for hybrid system with result comparison with traditional hysteresis control strategy to indicate the excellent performance of proposed optimization approach. This segment will be introduced in Chapter 6;

- Development of a PSOGA optimization method for hybrid system with experiment validation. And the comparison result between PSOGA and traditional hysteresis control strategy demonstrate the prominent implementation of PSOGA towards applied platform. This segment will be presented in Chapter 7 and the related work is under review. 


\subsection{Organization of the thesis}

The rest of the thesis is organized as follows: In Chapter 2, the fundamentals of neural networks, the basic training methods, the BP, the RBF, the SVM, the ELM, the particle swarm optimization, and the genetic algorithms, used for the modeling and optimization of hybrid system in this thesis, are briefly presented. The detailed introduction of principle of hybrid system as well as performance evaluation under different operating conditions is illustrated in Chapter 3. A series of experiment tests are conducted in order to investigate the effect of primary pressure, evaporating pressure and back pressure on COP and entrainment ratio; to investigate the ejector mixing chamber effect on COP by using ejectors with different geometry parameters and to compare the performance of both general mode and hybrid mode. Chapter 4 describes the hybrid model of the individual components in hybrid systems. Chapter 5 develops an ANN model for hybrid system, where the SLFN is trained with the extreme learning machine. The comparison between other training algorithms indicates the excellent performance of ELM. The energy consumption and system stability are modeled with SLFN trained by ELM for optimization in Chapter 6. In Chapter 7, a centralized optimization technique based on the PSOGA is proposed with the consideration of the system energy consumption and corresponding constraints. Chapter 8 briefly introduces the obtained conclusion and discuss about the future works. 


\section{Chapter 2 Artificial Neural Network Modeling and}

\section{Optimization Methods}

In this chapter, the method applied in the project is illustrated. Firstly, the artificial neural network is introduced in terms of its origin, patterns and structures. Then, different types of artificial neural network, such as radial basis function (RBF), support vector machine (SVM) and extreme learning machine (ELM) are explained in detail. After this, a feature selection algorithm is mentioned for data processing. At last, two optimization methods, namely genetic algorithm (GA) and particle swarm optimization (PSO) are presented.

\subsection{Introduction to Artificial Neural Networks}

\subsubsection{Biological neuron}

Since human brain is so omnipotent that it is able to apply what it learnt into practice, scientists try to make computer operating in the way that human brain behaves. The very first concept of artificial neural networks is inspired by the examinations of humans' central nervous systems. Like nerve cell is the fundamental unit of human brain, the neuron that is a simple artificial node in the artificial neural network is also the basic computational unit. According to statistical data, there are more than 9.5 billion neurons with interconnections within a human brain $[25,26]$.

As the design of artificial neural networks is inspired by biological neural networks, it is necessary to have a glimpse of biological neuron first. The structure of a typical biological neuron is shown in Figure 1, which consists of three parts: dendrites, soma and axon. Usually, dendrites represent the input stimulus, soma is the body part of nerve cell and axon is the connection path towards other nearby neurons. Normally, a neuron is surrounded by thousands of other neurons and is connected with them via its dendrite tree. When a stimulus is generated in a neighboring neuron, there will be a corresponding graded electro-genesis and with the help of dopamine, it will reach and be received by the neuron's dendrite tree. An electrical pulse will be generated if the charge level exceeds a critical value. And this pulse will be transmitted from soma to axon, further down to the next neuron. In this way, the biological neural network is working. Based on the operation principle of biological neural network, the artificial neural network is built up [27]-[29]. 


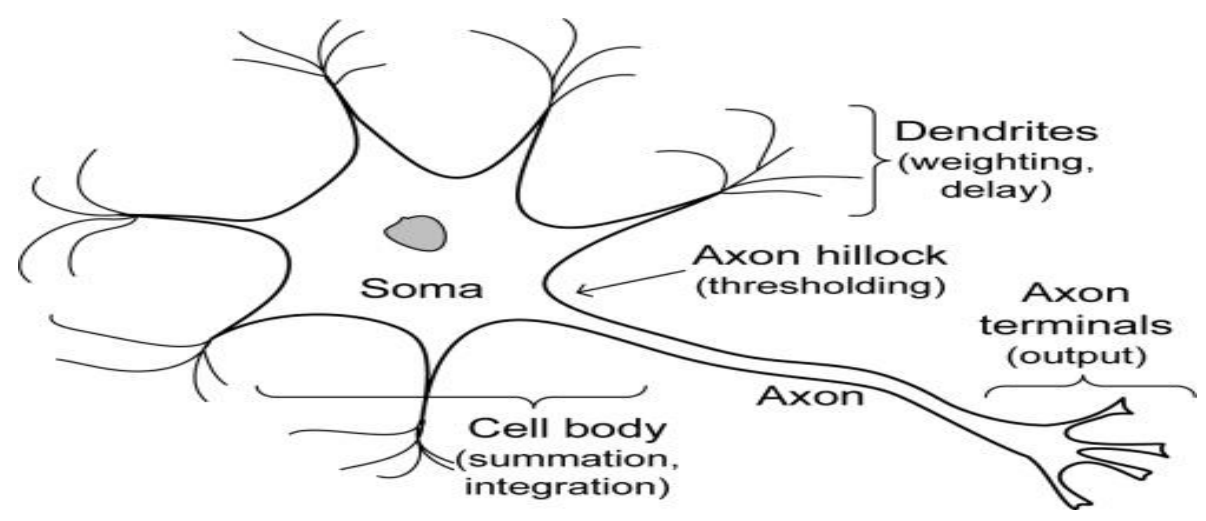

Figure 1 Schematic diagram of a biological neuron [29]

\subsubsection{Patterns of an artificial neuron}

Artificial neurons are the constitutive units in an artificial neural network and each neuron is a mathematical function conceived as a model to mimic biological neuron. Depending on the specific model used, there are many kinds of artificial neuron while the two most widely used neuron models are additive neuron and radial basis function (RBF) neuron.

Figure 2 shows the block diagram of an additive neuron model. And it can be seen that an additive neuron model consists of three basic elements [26]:

Synapses (the set of links in the diagram): Each synapse is accompanied with a certain value that is distinct from the synapse of biological neuron. This certain value is called the weight or strength of this synapse and may have positive or negative values depending on particular situation. The synaptic weight $w_{k i}$ means the weight between the input signal $i$ and the neuron $k$.

An adder: The purpose of the adder is to calculate the sum of weighted input signals.

An activation function: The aim of an activation function is to confine the amplitude of the signals produced by the adder and maintains the output value of the neuron within certain range. Typically, the output value of a neuron is normalized among the range of $[0$, $1]$ or $[-1,1]$ alternatively. 


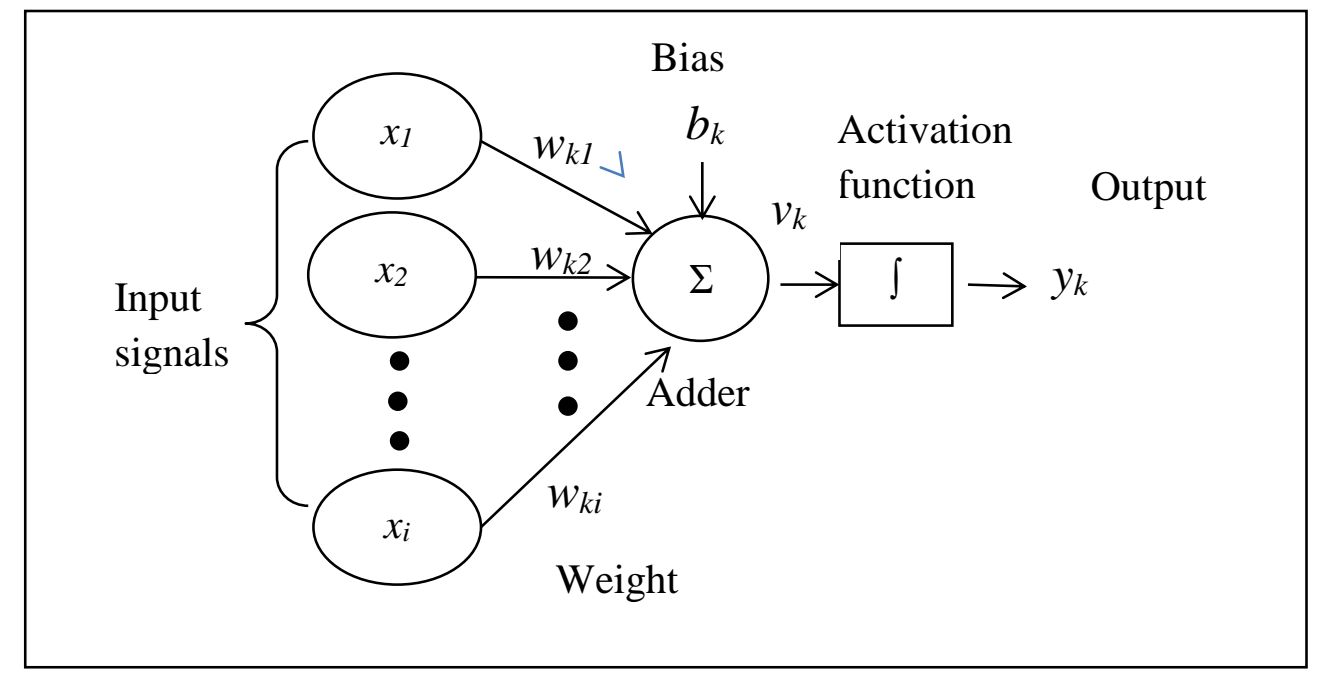

Figure 2 An additive neuron model

The detailed mathematical expression of an artificial neuron model is illustrated as follows [28]:

$$
y_{k}=f\left(\sum_{i=1}^{j} w_{k i} x_{i}+b_{k}\right)
$$

where $x_{i}$ is the input signals, $w_{k i}$ is the weight between input $i$ and neuron $k, b_{k}$ is the bias, $f$ represents the activation function and $y_{k}$ is the output value of the neuron $k$.

There are many kinds of activation functions and four typical ones are shown as follows:

Step function

$$
f(t)= \begin{cases}1 & t \geq 0 \\ 0 & t<0\end{cases}
$$

Piecewise linear function

$$
f(t)=\left\{\begin{array}{cc}
1 & t \geq 1 \\
\tau & 0<t<1 \\
0 & t \leq 0
\end{array}\right.
$$

Log sigmoid function

$$
f(t)=\frac{1}{1+\exp (-t)}
$$

Tan sigmoid function 


$$
f(t)=\frac{1-\exp (-2 t)}{1+\exp (-2 t)}
$$

Different from additive neurons, a typical RBF neuron is majorly made up of three segments: synapses that receive input signals, activation functions and output signals. The block diagram of an RBF neuron model is shown in Figure 3. Normally, the synaptic weights between input signals and activation functions are set to be 1 by default. Additional, a distance calculator is embedded into the activation function to evaluate the distance from the origin. And this origin, which is called the center of the neuron $\boldsymbol{c}_{i}$, is introduced in the RBF neuron model. In most cases, Euclidean distance is usually applied to calculate the distance between the input vector and center of the neuron, although other distance functions are also possible.

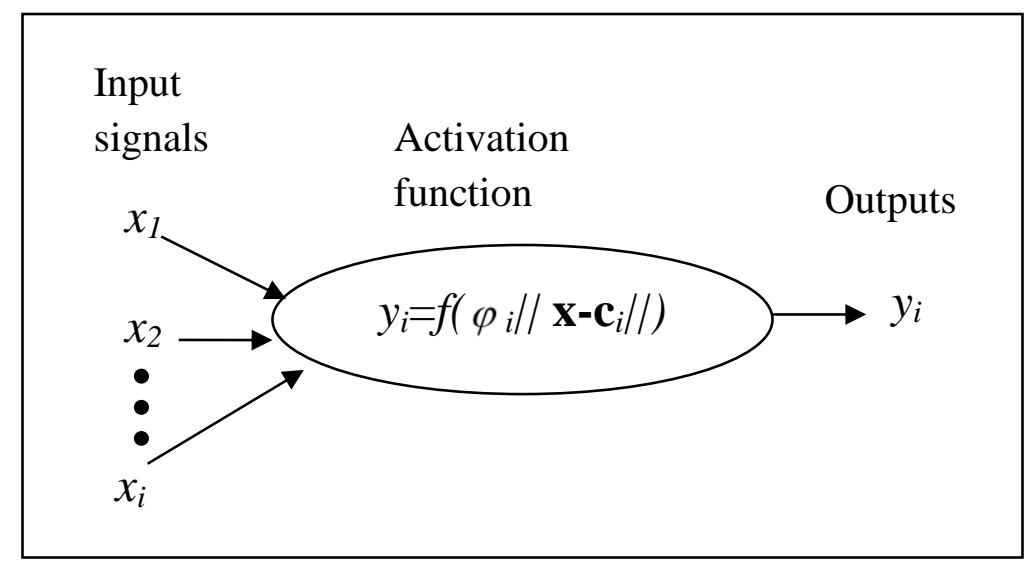

Figure 3 A RBF neuron model

For RBF neurons, the model can be expressed mathematically as follows:

$$
y_{i}=f\left(\varphi_{i}\left\|x-c_{i}\right\|\right)
$$

where $\mathbf{x}$ is the input signal in vector form, $\boldsymbol{c}_{i}$ indicates the center of neuron, $g($.$) is the$ activation function which Gaussian function is usually applied and $y_{i}$ is the output signal. Although Gaussian function is commonly used, other functions may also give good performance under particular conditions and they are all shown as follows:

$$
\begin{gathered}
f(\mathrm{x})=\exp \left(-\beta\|x-c\|^{2}\right) \\
f(\mathrm{x})= \pm \sqrt{\|x-c\|^{2}+\gamma}
\end{gathered}
$$




$$
f(\mathrm{x})=\|x-c\|^{2} \times \log \left(\|x-c\|^{2}\right)
$$

\subsubsection{Structures of neuron network}

There are many criterions for neuron network classification. For instance, according to learning algorithm, it can be classified as supervised learning network and unsupervised learning network while according to network connectionism, it can be divided into feedforward network, recurrent network and reinforcement network [30]. Since feedforward network is the most popular and widely used one, it is emphatically described in this section. For feedforward network, there are two kinds of structure, namely single layer feedforward network and multi-layer feedforward network. In general, the feedforward networks consist of three basic layers: input layer, hidden layers and output layer.

Single-Layer Feedforward Networks

From the literal meaning, it can be concluded that there is only one hidden layer in the feed-forward networks. The graphic description about the network is shown in Figure 4.

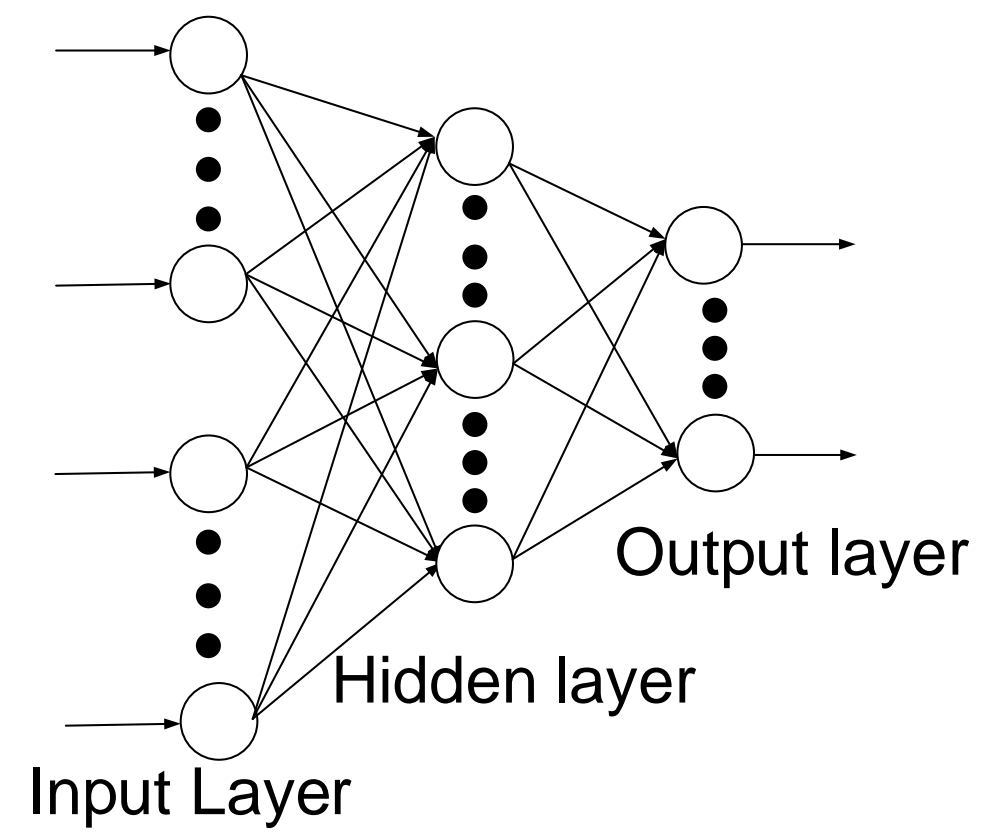

Figure 4 Structure of a Single Layer Feed-forward Neural Network

Multi-Layer Feedforward Networks 
From the literal meaning, there are more than one hidden layers in the feedforward networks. The benefit of introducing these extra hidden layers is that it can help improve the capacity of network, which in turn enable the network to handle the issue like higherdimensional nonlinear classification. Signals now need to travel more than one hidden layer until it reaches the output layer. The output signals of the former hidden layer are used as the input signals of the latter hidden layer, and so on. The final output that reflects the overall response of the network is produced by output layer. Figure 5 presents a multi -layer feedforward network.

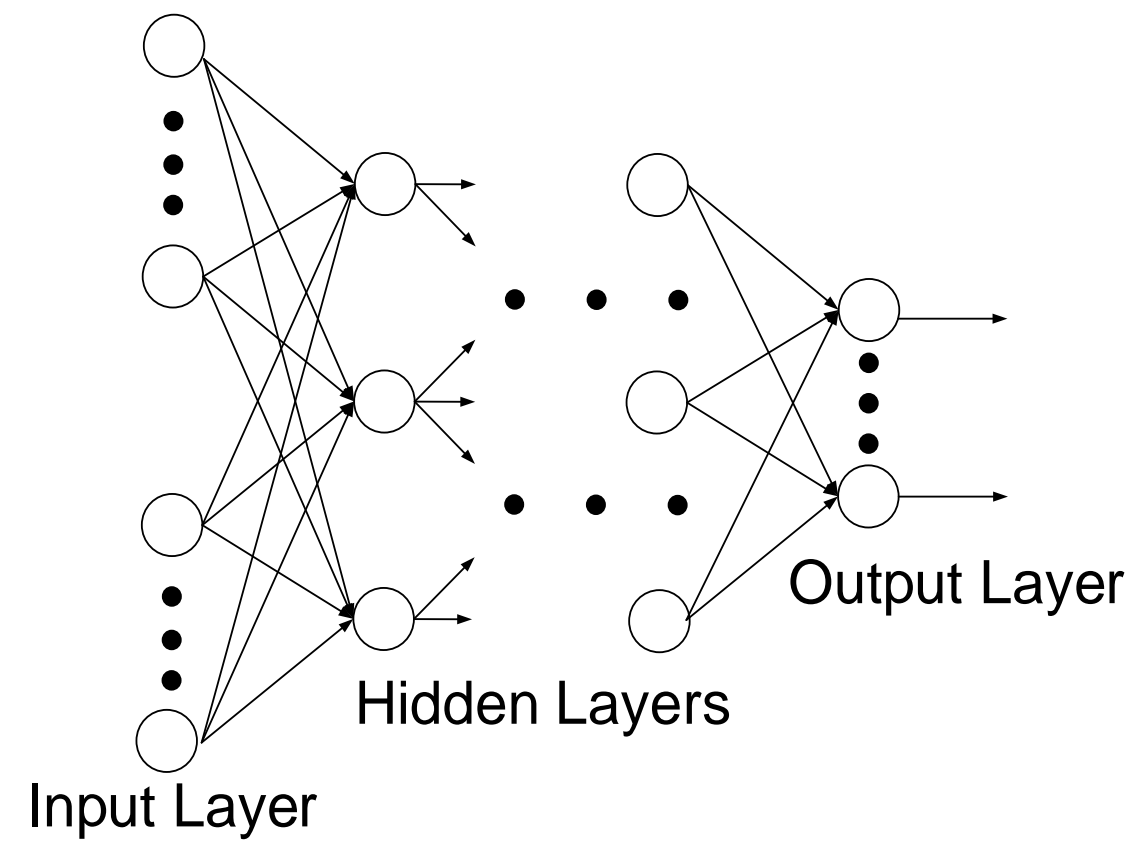

Figure 5 Structure of a Multi-layer Feed-forward Neural Network

Besides, learning algorithms are essential to a neural network. The objective of learning algorithms is to minimize the cost criterion so that the performance of network is optimized. There are numerous algorithms available for neural network training and gradient descent learning algorithms are the most commonly used among others for multilayer networks training. Gradient descent is also known as steepest descent and is a first order optimization algorithm. The idea is to take the steps proportional to the negative (or positive) of the gradient in order to find a local minimum (or a local maximum) of a function. Among all the gradient descent learning algorithms, back propagation algorithm is one of the most popular algorithms. Back propagation is an 
abbreviation for "backward propagation of errors" and it is a common method of training artificial neural networks. The method is used in supervised learning and calculated the derivative of difference between target and actual value with respect to all the weights in the network. Normally, the learning algorithm can be divided into two phases: propagation part and weight update part. During the propagation process, the input of training pattern is forward propagated through the neural network layer by layer so that to generate the corresponding output activations; the difference between the targeted value and actual output is backward propagated through the neural network to produce correction in hidden neurons. For the weight update process, the difference is multiplied by input signal to obtain the gradient of the weight and then the newly weight is a result of subtract a percentage of the gradient from the former weight. This percentage is called the learning rate and it influences the performance of the algorithm in terms of speed and quality. The greater the ratio, the faster the training speed; while the lower the ratio, the more accurate the training is. It is a trade-off between accuracy and efficiency. This process does not stop until the terminate criterion is satisfied.

The cost function of the network is obtained by summation of the squared errors, and the weights update process is based on the formula below [31].

$$
w(n+1)=w(n)-\eta \Delta E(n)=w(n)-\eta \frac{\partial E(n)}{\partial w}
$$

where $\eta$ is the learning rate, $w(n)$ is the weight at the $n$th iteration and $\Delta E(n)$ represents the gradient of cost function at the $n$th iteration.

Due to the fact that in BP algorithm, the weight needs to be updated iteratively, thus the time taken for convergence is usually quite long, especially under the condition that a large number of hidden neurons in the network. Additional, it is highly possible that the result provided by BP algorithm traps to local minima for nonlinear optimization problem or the efficiency could be low for flat regions or even worse it will never stop.

\subsection{Radial Basis Function Network (RBF)}

\section{Background illustration}

Before introducing the RBF network, it is necessary to talk about radial basis function first. This function was introduced by Powell in 1985. And in essence, it is a scalar function which is symmetric along the radial direction. Generally, it is a monotonous 
function defined by the Euclidean distance between a random point $\mathrm{x}$ and a certain center c within a space, and can be written as $k(\|x \quad c\|)$ where the impact is usually localized, which means that when $\mathrm{x}$ is far away from $\mathrm{c}$, the value of function is very small.

The introduction of radial basis function is mainly to solve multivariable interpolation problem. For better illustration, the process is shown in Figure 6.
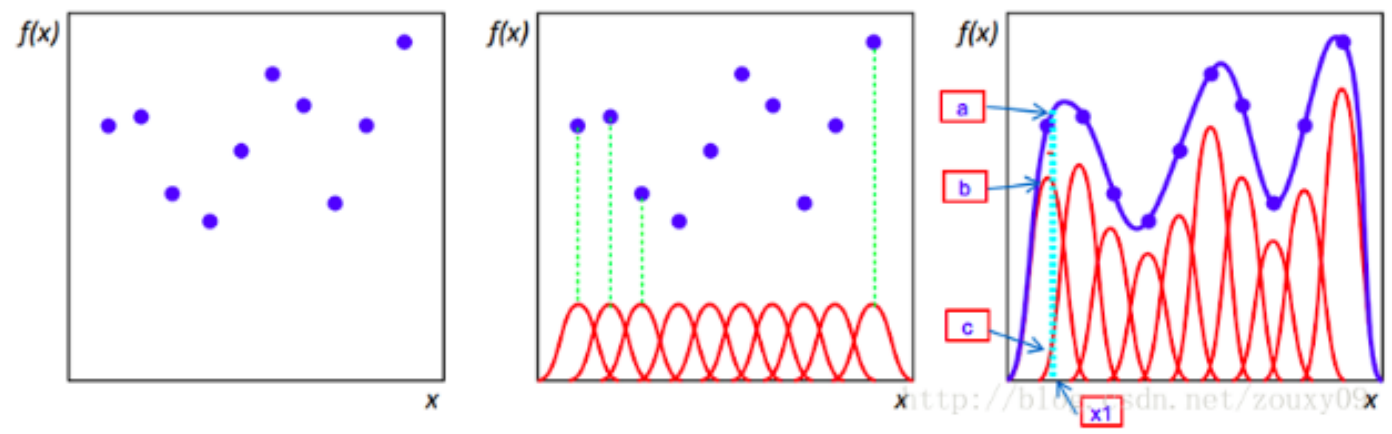

Figure 6 The application of radial basis function

In the left figure, each blue point represents a sample. And in the middle one, radial basis function is applied to every sample. In this case, the center of each Gaussian function is the sample itself. The green dot line indicates the value on radial basis function corresponding to particular sample. In the right one, the blue line is the final regression result. If we want to know the corresponding value of a new data point, namely $\mathrm{x} 1$, it can be obtained by adding the value of $b$ and $c$. Because the other sample point is too far from $\mathrm{x} 1$, and there impact are zeros.

In 1988, the Radial Basis Function networks were first formulated by [32, 33], the researcher at the Royal Signals and Radar Establishment. It is a feed forward network with radial basis functions as activation function and can approach any continuous function within any precision. Radial basis function networks have many applications, such as function approximation, classification, time series prediction and system control. Due to its outstanding performance in data mining issues, more and more researchers put their efforts in this field [34].

The structure of RBF network shares similar things with that of multi-layer feed forward network. It is a three layers feed forward network, namely, an input layer, a hidden layer where the number of hidden neuron is determined by particular problem and the activation function is radial basis function which is a non-negative non-linear function of radial symmetry and attenuation of center points, and a linear output layer. The structure diagram of RBF network is shown in Figure 7. 


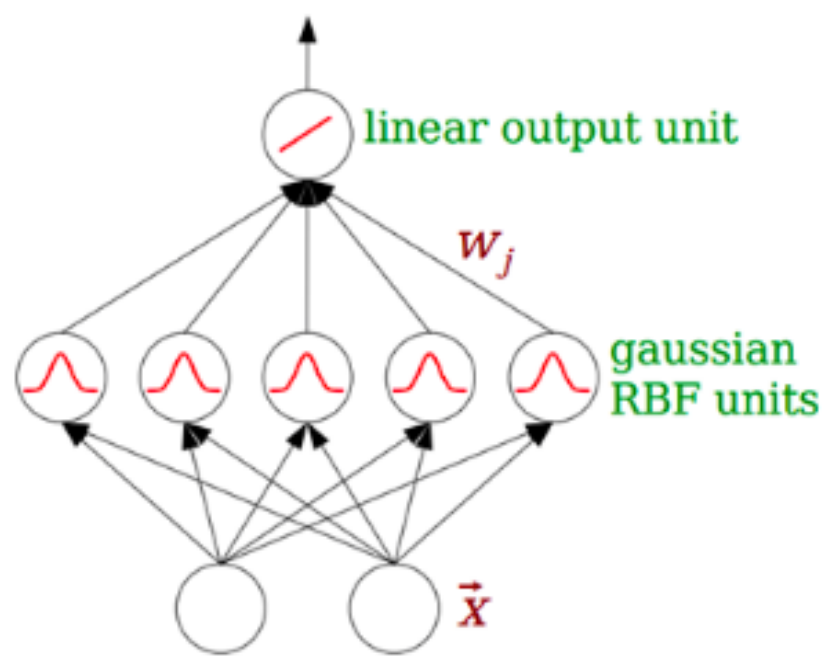

Figure 7 The diagram of RBF network

The mathematical expression of the network is given by

$$
\varphi(x)=\sum_{i=1}^{N} w_{i} \rho\left(\left\|x-c_{i}\right\|\right)
$$

where $N$ is the number of hidden neurons, $c_{\mathrm{i}}$ is the center vector for neuron $i$ and $w_{\mathrm{i}}$ is the weight of neuron $i$ in the linear output neuron. Euclidean distance is typically applied and the Gaussian function is often taken as radial basis function, thus the mathematical form is

$$
\rho\left(\left\|x-c_{i}\right\|\right)=\exp \left(-\beta\left\|x-c_{i}\right\|^{2}\right)
$$

RBF networks are universal approximators on a compact subset of $\mathrm{R}^{\mathrm{n}}$ with certain mild conditions on the shape of the activation function. This also means that if given enough hidden neurons, RBF network can approximate any continuous function with arbitrary precision.

\section{Training procedure}

For function fitting with ordinary complexity or classification purpose, the choice for centers is intricate beyond imagination since no clue exists apparently. The training is typically accomplished in two steps: width centers fixing and weights adjustment. This is due to the different inherited behavior of the non-linear characteristic of hidden neurons with regard to the linear behavior of output neuron.

The centers of radial basis function can be either randomly selected from input instances or acquired through using orthogonal least square algorithm or by clustering the samples and then taking the cluster means as the center $[35,36]$. 
The widths are usually all immobilized to identical value that is proportional to the maximum distance between the chosen centers.

For linear weights adjustment, there are many training algorithm available. Three different types of solutions are introduced in the following [37].

\section{Pseudo-inverse solution}

If the centers $c_{\mathrm{i}}$ have been fixed, to obtain the weights that minimize the output error, a linear pseudo-inverse solution is applied and computed as:

$$
=H^{+} d
$$

where the entries of $\mathrm{H}$ are the values of the radial basis functions evaluated at the points $x_{\mathrm{i}}$ that

$$
g_{j i}=\rho\left(\left\|x_{j}-c_{i}\right\|\right)
$$

Unlike multi-layer perceptron networks, RBF networks have a unique local minimum with the centers are fixed.

\section{Gradient descent solution}

As mentioned before, the weights are adjusted at each time step by guiding them in a direction opposite to the gradient of the objective function.

$$
\boldsymbol{w}(t+1)=\boldsymbol{w}(t)-\eta \frac{d}{d \boldsymbol{w}} H_{t}(\boldsymbol{w})
$$

For the case of training the linear weights, $a_{\mathrm{i}}$, the algorithm becomes

$$
a_{i}(t+1)=a_{i}(t)+\eta[y(t)-\varphi(\boldsymbol{x}(t), \boldsymbol{w})] \rho\left(\left\|x(t)-c_{i}\right\|\right)
$$

\section{Projection operator solution}

For the case of training the linear weights, $a_{\mathrm{i}}$, the algorithm is like:

$$
a_{i}(t+1)=a_{i}(t)+\eta[y(t)-\varphi(\boldsymbol{x}(t), \boldsymbol{w})] \frac{\rho\left(\left\|x(t)-c_{i}\right\|\right)}{\sum_{i=1}^{N} \rho^{2}\left(\left\|x(t)-c_{i}\right\|\right)}
$$

The projection operator training becomes Newton's method if there is one basis function. In this case, the centers are determined with both randomly generated and the K-means clustering method, while the gradient descent method is applied for weight training. 


\subsection{Support Vector Machine (SVM)}

\section{Background illustration}

The Support Vector Machine (SVM) was first proposed by [38, 39] in 1963 and then modified to create nonlinear classifiers by applying the kernel trick to maximum-margin hyper-planes. The current standard soft margin form was developed by [40] in 1993. SVMs are supervised learning models with associated algorithms that deal with classification and regression issues. Due to the excellent performance of the derived kernel methods, more and more attentions have been drawn onto the research about SVM.

\section{Training procedure}

Generally speaking, the most common use for SVM is to recognize differences among patterns. The crux element beneath SVM is that a hyper-plane or set of hyper-planes is constructed in a high or infinite dimensional space to maximize the margin of separation between different classes. The feature of an achieved good separation is that the hyperplane constructed has the largest distance to the nearest training data point of any class, in other words, the largest functional margin. The larger the margin is, the lower the classifier error is [41]. According to real situation, the expression of SVM can be divided into two manners which are as follows:

\section{Hard margin}

If given a training dataset that contains $n$ points $\left\{\left(\mathbf{x}_{i}, y_{i}\right)\right\}_{i=1}^{n}$, where the value of $y_{i}$ are either 1 or $-1, \mathbf{x}_{i} \in \mathbf{R}^{d}$ is an input vector related with each $y_{i}$ for $i=1, \ldots, n$. A decision hyperplane needs be able to divide the group of points $\mathbf{x}_{i}$ with $y_{i}=1$ from the group of points for which $y_{i}=-1$. Also the distance between the hyper-plane and the nearest point $\mathbf{x}_{i}$ from either group is maximized. The above mentioned hyper-plane can be expressed as follow

$$
\boldsymbol{w} \cdot \boldsymbol{x}-b=0
$$

where $\boldsymbol{w}$ is the normal weight vector to the hyper-plane and $b$ is the bias which helps determine the offset of the hyper-plane from the origin along the normal vector $\boldsymbol{w}$. Since it is a two class classification problem, the sign function can be applied to denote the input pattern and it is shown as follows:

$$
f(x)=\operatorname{sgn}(w \cdot x-b)
$$


where

$$
\operatorname{sgn}(t)=\left\{\begin{array}{cc}
1 & t \geq 0 \\
-1 & t<0
\end{array}\right.
$$

Geometrically speaking, the distance between the proposed hyper-planes is $\frac{2}{\|\omega\|}$, so the maximum distance can be achieved under the minimum value of $\|\omega\|$. The constraint can be described as follow.

$$
y_{i}\left(w \cdot x_{i}-b\right) \geq 1 \quad \text { for all } i=1, \ldots, n
$$

Combine with the demand stated before, the optimization problem becomes:

$$
\begin{aligned}
& \min \|\omega\| \\
& \text { s.t. } \quad y_{i}\left(w \cdot x_{i}-b\right) \geq 1 \quad \text { for } i=1, \ldots, n
\end{aligned}
$$

\section{Soft margin}

The above hard margin is under the assumption that there exists such hyper-plane that can successfully separate different pattern set without any error. Unfortunately, in real situation, this kind of hyper-plane merely exists. Thus, a soft margin which is able to allow a small amount of error in classification is desirable. Therefore, the hinge loss function is introduced as

$$
\max \left(0,1-y_{i}\left(\boldsymbol{w} \cdot \boldsymbol{x}_{i}-b\right)\right)
$$

If $\boldsymbol{x}_{i}$ is located on the correct side of the margin, the function will be zero. While it lies on the wrong side, the value of function is proportional to the distance away from the margin. So, the problem then becomes to minimize the expression

$$
\left[\frac{1}{n} \sum_{i=1}^{n} \max \left(0,1-y_{i}\left(\boldsymbol{w} \cdot \boldsymbol{x}_{i}-\mathrm{b}\right)\right)\right]+\lambda\|\omega\|^{2}
$$

There is a tradeoff between increasing the margin size and guaranteeing the correct classification probability through varying parameter $\lambda$. The smaller the $\lambda$ is, the closer behavior towards hard margin. Furthermore, to minimize Eq. (24), it can be rewritten as a constrained optimization problem. Before this, a non-negative slacking variable $\xi_{i}$ is introduced and it equals to $\xi_{i}=\max \left(0,1-y_{i}\left(\mathbf{w} \cdot \mathbf{x}_{i}-b\right)\right)$. Thus, the rewritten problem is shown as follows: 


$$
\begin{aligned}
& \min \lambda\|\omega\|^{2}+\frac{1}{n} \sum_{i=1}^{n} \xi_{i} \\
& \text { s.t. } \quad y_{i}\left(w \cdot x_{i}-b\right) \geq 1-\xi_{i} \quad \text { for } i=1, \ldots, n \\
& \xi_{i} \geq 0
\end{aligned}
$$

This is the primal problem which can be solved by transferring it into Lagrangian dual form. So the simplified problem can be obtained as

$$
\begin{aligned}
& \max f\left(c_{1}, \ldots c_{n}\right)=\sum_{i=1}^{n} c_{i}-\frac{1}{2} \sum_{i=1}^{n} \sum_{j=1}^{n} y_{i} c_{i}\left(x_{i} \cdot x_{j}\right) y_{j} c_{j} \\
& \text { s.t. } \sum_{i=1}^{n} c_{i} y_{i}=0 \quad \text { for } i=1, \ldots, n \\
& 0 \leq c_{i} \leq \frac{1}{2 n \lambda}
\end{aligned}
$$

Due to the fact that the dual minimization problem is a quadratic function, the quadratic programming algorithms can be applied to solve this problem. The variables $c_{i}$ satisfy such relationship as

$$
w=\sum_{i=1}^{n} c_{i} y_{i} x_{i}
$$

If $c_{i}=0$, that means the classification is correct for point $x_{i}$, while if $0<c_{i}<(2 n \lambda)^{-1}, x_{i}$ lies on the boundary of margin. The value of $b$ can be easily obtained by calculating any margin point $x_{\mathrm{i}}$

$$
b=y_{i}-w \cdot x_{i}
$$

Finally, the discriminative function is given by

$$
f(\mathrm{x})=\operatorname{sgn}(\boldsymbol{w} \cdot \boldsymbol{x}-\mathrm{b})=\operatorname{sgn}\left(\sum_{i=1}^{n} c_{i} y_{i} x_{i}^{T} x-b\right)
$$

Since in real classification assignment, the Lagrange multipliers $c_{i}$ merely tend to surpass zero. The function $f(\mathrm{x})$ depends solo on the respective training vectors which is regarded as support vectors.

The nonlinear SVMs have their merits on dealing with certain problems. Whereas the original problem is stated in a finite dimensional space, the proposed approach can achieve the effect that the original finite dimensional input vectors be mapped into a 
higher dimensional feature space, presumably simplifying the separation process [42]. The original problem can be transferred into a linear separable problem by applying the nonlinear mapping $\Phi$. Then it can be solved with linear SVMs method. The nonlinear mapping $\Phi$ is often appeared as kernel function $K$. Some commonly used kernel functions are shown as follow including the Gaussian kernel which is the most popular one.

$$
\begin{aligned}
& \text { Polynomial } K\left(x_{i}, x_{j}\right)=\left(x_{i}, x_{j}\right)^{d} \\
& \text { Gaussian } K\left(x_{i}, x_{j}\right)=\exp \left(-\gamma\left\|x_{i}-x_{j}\right\|^{2}\right) \\
& \text { Hyperbolic tangent } K\left(x_{i}, x_{j}\right)=\tanh \left(\kappa x_{i} \cdot x_{j}+c\right)
\end{aligned}
$$

Finally, the SVM function can be mathematically expressed as

$$
f(x)=\operatorname{sgn}\left(\sum_{i=1}^{n} c_{i} y_{i} K\left(\boldsymbol{x}_{i} \cdot \boldsymbol{x}\right)-b\right)
$$

\subsection{Feedforward Neural Networks With Random Weights}

\section{Background illustration}

The feed-forward neural networks with random weights are introduced by [43] that offer

some experimental understanding about reason behind the less importance of weights of neural network.

\section{Training procedure}

The structure of proposed feed-forward neural network is shown in Figure 8. 


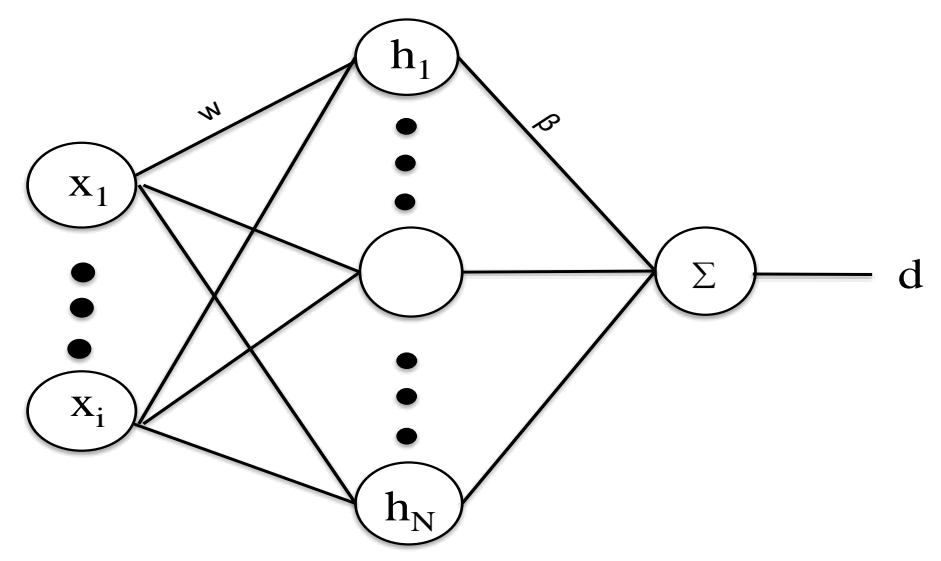

Figure 8 Structure of a feed-forward neural network with random weights

From Figure 8, it can be observed that the proposed network consists of a single hidden layer, and there is no feed-back in the system. The activation function applied in the hidden layer is sigmoid function which maps the input in the range between 0 and 1 . In mathematical formulation, the process can be described as:

$$
H=g\left(W^{T} x\right)
$$

Here $\mathrm{W}$ and $\mathrm{X}$ are the weight and input vectors, respectively.

Then, the output is calculated as:

$$
d=\sum \beta\left(W^{T} x\right)
$$

So, the weight are optimized by using the well-known L2 criterion:

$$
E^{2}=\sum\left(d-\sum \beta\left(W^{T} x\right)\right)^{2}=\sum\left(d-\beta^{T} H\right)^{2}
$$

The weight vector $\beta^{*}$ for which this equation is minimal is calculated as follows:

$$
\frac{\partial E^{2}}{\partial \beta}=2 R \beta^{*}-2 P=0
$$

therefore,

$$
\beta^{*}=R^{-1} P
$$


where

$$
\begin{aligned}
& R=\sum H H^{T} \\
& P=\sum d H^{T}
\end{aligned}
$$

$\mathrm{R}$ is the input correlation matrix and $\mathrm{P}$ is the input-target correlation vector, respectively.

The author conducted experiments which indicate that these weights can be taken randomly, for a number of non trivial problem.

\subsection{The Extreme Learning Machines (ELM)}

\section{Background illustration}

The extreme learning machines are kind of feedforward neural network with a single hidden layer. The most distinct feature of it is that the weights connecting inputs and hidden neurons are randomly selected and never updated [44, 45]. Thanks to this setting, the training efficiency is improved tremendously (usually thousands of times faster than BP training) while maintaining a good generalization performance. ELMs can be used for dealing with classification and regression problem.

\section{Training procedure}

Next, let's take a deep look at the basic single layer feedforward neural network. Given $N$ training data pairs $\left(X_{i}, d_{i}\right)$ that are measured and recorded from an experimental platform, where $X_{i}$ is the input pattern in vector form and $d_{i}$ indicates the $i$ th desired output vector. The two kinds of vectors are defined detailed as $X_{i}=\left[\begin{array}{llll}x_{i 1} & x_{i 2} & \ldots & x_{i n}\end{array}\right]^{T}$ and $d_{i}=\left[\begin{array}{llll}d_{i 1} & d_{i 2} & \ldots & d_{i m}\end{array}\right]^{T}$, for $i=1,2, \cdots N$. Substitute the given input and output into the basic single layer feedforward network for training, the structure of it can be displayed in Figure 9: 


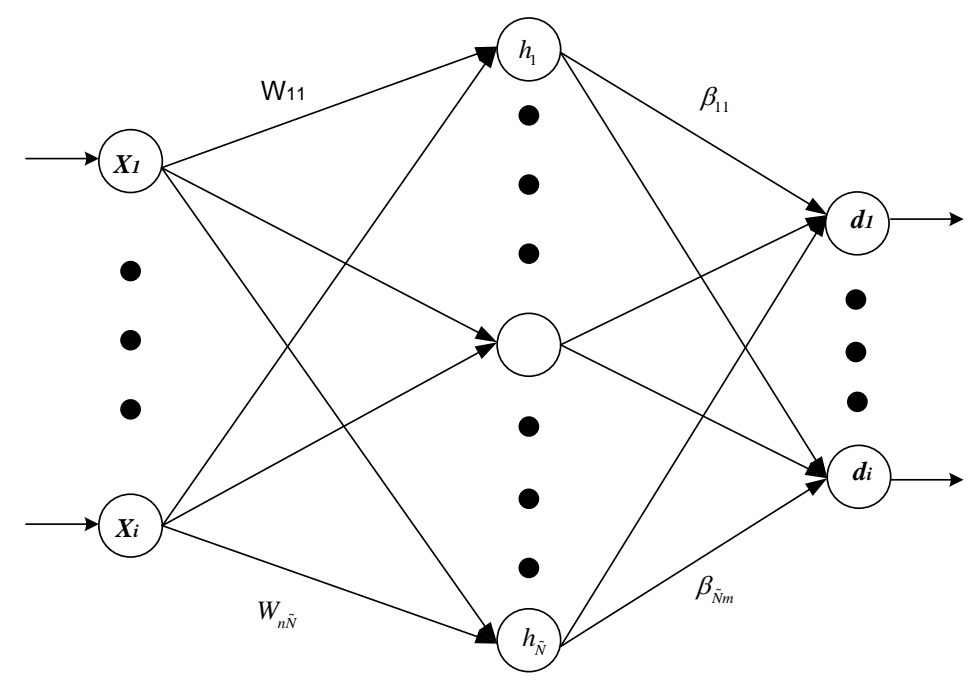

Figure 9 Structure of a basic single hidden layer feed-forward neural network

where the input and output nodes are linear, and the hidden nodes are of nonlinear activation functions, shown as

$$
h_{j i}=g\left(W_{j}^{T} x_{i}\right)
$$

where $W_{j}$ is the weight vector connected between input and the $j$ th hidden neuron, expressed as:

$$
W_{j}=\left[\begin{array}{llll}
W_{1 j} & W_{2 j} & \ldots & W_{n j}
\end{array}\right]^{T}
$$

for $j=1,2, \cdots \tilde{N}$, and according to ELM principle, the values of input weights in $W_{j}$ are all randomly assigned.

Then the vector form of hidden layer output $H_{i}$, which corresponding to $X_{i}$, can be expressed as:

$$
\begin{aligned}
H_{i} & =\left[\begin{array}{lll}
h_{1 i} h_{2 i} \ldots & h_{\tilde{N} i}
\end{array}\right]^{T} \\
& =\left[\begin{array}{llll}
g\left(W_{1}^{T} X_{i}\right) & g\left(W_{2}^{T} X_{i}\right) & \cdots & g\left(W_{\tilde{N}}^{T} X_{i}\right)
\end{array}\right]^{T}
\end{aligned}
$$

Based on formal equations, the $k$ th output of the network can be obtained as:

$$
Y_{k i}=\sum_{l=1}^{\tilde{N}} \beta_{l k} g\left(W_{l}^{T} X_{i}\right) \quad k=1,2, \cdots, m
$$


Furthermore, the $i$ th output of network in vector form is written as:

$$
\begin{aligned}
Y_{i} & =\left[\begin{array}{llll}
Y_{1 i} & Y_{2 i} & \cdots & Y_{m i}
\end{array}\right]^{T} \\
& =\left[\begin{array}{lllll}
\sum_{l=1}^{\tilde{N}} \beta_{l 1} g\left(W_{l}^{T} X_{i}\right) & \sum_{l=1}^{\tilde{N}} \beta_{l 2} g\left(W_{l}^{T} X_{i}\right) & \cdots & \sum_{l=1}^{\tilde{N}} \beta_{l m} g\left(W_{l}^{T} X_{i}\right)
\end{array}\right]^{T}
\end{aligned}
$$

Thus, the overall output vectors, which correspond to the training vectors, can be rewritten in the matrix form and is displayed as:

$$
\begin{aligned}
Y & =\left[\begin{array}{llll}
Y_{1} & Y_{2} \cdots Y_{N}
\end{array}\right] \\
& =\left[\begin{array}{cccc}
\sum_{l=1}^{\tilde{N}} \beta_{l 1} g\left(W_{l}^{T} X_{1}\right) & \sum_{l=1}^{\tilde{N}} \beta_{l 1} g\left(W_{l}^{T} X_{2}\right) & \cdots & \sum_{l=1}^{\tilde{N}} \beta_{l 1} g\left(W_{l}^{T} X_{N}\right) \\
\vdots & \vdots & \cdots & \vdots \\
\sum_{l=1}^{\tilde{N}} \beta_{l m} g\left(W_{l}^{T} X_{1}\right) & \sum_{l=1}^{\tilde{N}} \beta_{l m} g\left(W_{l}^{T} X_{2}\right) & \cdots & \sum_{l=1}^{\tilde{N}} \beta_{l m} g\left(W_{l}^{T} X_{N}\right)
\end{array}\right] \\
& =\beta^{T} H
\end{aligned}
$$

where

$$
\begin{gathered}
\beta_{i}=\left[\begin{array}{llll}
\beta_{1 i} & \beta_{2 i} & \cdots & \beta_{\tilde{N} i}
\end{array}\right]^{T} \quad i=1,2, \cdots m \\
H_{i}=\left[\begin{array}{llll}
g\left(W_{1}^{T} X_{i}\right) & g\left(W_{2}^{T} X_{i}\right) & \cdots & g\left(W_{\tilde{N}}^{T} X_{i}\right)
\end{array}\right]^{T} \quad i=1,2, \cdots N
\end{gathered}
$$

The matrix $H$ is called the hidden layer output matrix of the neural network. There are two different situations about $H$ that is whether it is square or non-square. If it is square matrix, it must satisfy the condition that the number of hidden nodes and the number of training samples are equal. Besides, all the training samples must be linearly independent with each other which can be derived by random selecting the weights of hidden layer. Under this ideal condition, ELM can perfectly approximate the training samples without any deviation [46]. But in real application, the number of hidden nodes is usually smaller compared with number of training sample. This leads to the fact that $H$ is non-square for majority of time. To find the least square solution with smallest norm, the Moore Penrose generalized inverse need to be introduced [47]. And the definition is as follow:

Given a general linear system $A x=y$, where $A$ may be singular or even non-square. A matrix $G$ of order $n \times m$ is the Moore-Penrose generalize inverse of matrix $A$ of order $m \times n$, if 


$$
A G A=A \quad G A G=G(A G)^{T}=A G(G A)^{T}=G A
$$

The Moore Penrose generalize inverse of matrix $A$ will be denoted as $A^{+}$for convention. So there exists a matrix $P$ such that $P y$ is the least square solution of the linear system $A x=y$ with minimum norm. Additional, it is necessary and sufficient that $P=A^{+}$, the Moore Penrose generalize inverse of matrix $A$.

Hence, the solution of the above system is obtained as:

$$
\hat{\beta}=H^{+} d^{T}
$$

where $\mathrm{H}^{+}$is the Moore Penrose generalized inverse.

The general process of ELM is stated as following:

Firstly, the network is initialized (such as number of hidden nodes) with given training samples; then the hidden layer output matrix is calculated based on the randomly generated weights and bias, and lastly, the output weight is obtained based on equation (47).

\subsection{Feature Selection Algorithm}

The above mentioned regression methods are belonged to machine learning. Generally, when dealing with real problem, there may be either redundant or irrelevant features contained in the raw data. In order to improve the efficiency and accuracy of modeling process, it is necessary to identify and remove such features [48]. Thus, feature selection, or known as variable selection, is the process aims at selecting the relevant features for modeling construction purpose. There are three merits which feature selection techniques bring: simplification of models, shorten training times and performance enhancement with over-fitting reduction.

There are several common approaches to feature selection, boosted decision trees is one of them. The boosted tree applied follows the fashion of gradient boosting and it is used for feature ranking $[49,50]$. Several advantages are possessed by boosted trees, for instance, there is no requirement on normalization when dealing with categorical or count data, also by adjusting the number of trees, the trade-off between operating time and accuracy can be simply implemented. 
A sorted list of features with their relative importance is generated when applying boosted tree model and the relative influence of feature $x_{i}$ for single decision tree is expressed as:

$$
\widehat{J}_{i}^{2}=\frac{1}{N} \sum_{n=1}^{N} \widehat{J}_{i}^{2}\left(T_{n}\right)
$$

where $N$ is the number of trees. Further, the relative importance for each tree is obtained as:

$$
\widehat{J}_{i}^{2}(T)=\sum_{t=1}^{L-1} \widehat{I}_{t}^{2} 1\left(v_{t}=i\right)
$$

where $L$ is the number of terminal node of tree $T, t$ is the number of internal nodes, $v_{t}$ is the splitting feature that associated with node $t$ and $\widehat{I}_{t}^{2}$ is the squared error that reflects the improvement on split.

\subsection{Genetic Algorithm}

Genetic algorithm is an artificial intelligent search heuristic that inspired from the process of natural selection or the Darwin's evolution theory-survival of the fittest. It is commonly used to optimize certain problem within a confined region. To trace back the history of genetic algorithm, [51] is the person cannot be neglected since it was through his book "Adaptation in Natural and Artificial Systems" that genetic algorithms became popular. After this, Goldberg had made a big contribution in developing the genetic algorithm. Now, genetic algorithm has been studied for several decades and is widely used in dealing with complex problems including timetabling and scheduling problems. Since genetic algorithm belongs to evolutionary algorithm, the terminology applied in genetic algorithm sounds like biology, such as mutation, selection and crossover [52, 53, 54].

The detailed introduction of these terminologies is illustrated as follow.

Chromosome: In biological realm, a chromosome is a packaged and organized structure that contains most information of the DNA of a living organism. While in genetic algorithm, a matrix which consists of useful variables waited to be conducted represents the chromosome. They are coded in certain ways within a specified length of bits. The different types of coding will be discussed later. A single row of the matrix contains all the variables and each row indicates a new individual of the sample population. 
Population: For a certain species, the population means the total number of organisms of that species. Hence, in genetic algorithm, population indicates the number of individual in the sample. Since each row represents a new individual, the number of rows of the chromosome matrix equals to the size of sample population.

Generation: Genetic algorithm is an iterative calculation process, the evolution begins with the population that randomly generated and called parent. The population generated in each iteration is called a generation. The later generation is called the offspring of the former one. Usually, there is a maximum generation number defined by the user to prevent the algorithm from infinite looping.

Fitness function: For an optimization problem, the objective function is the goal to be achieved. In the fields of genetic algorithm, the objective function is transferred into a particular pattern, called fitness function. Fitness function reflects the closeness or fitness of the individual in the population. It is very important since the practical optimization problem is connected to genetic algorithm via fitness function. For instance, given a minimization problem, the individual chromosome with the lowest value of objective function is more appreciable. The fitness function is often expressed mathematically as a linear array of individuals with corresponding criteria:

$$
F(x)=f(o(x))
$$

where $F(x)$ is the fitness function, $o(x)$ is the objective function and $f($.$) is the transformer$ that convert objective function into fitness function.

However, in many cases, it is not easy to straightforward define the fitness function and even in some cases, it is impossible to even guess what fitness function might be. Thus, the difficulty largely depends on the real situation.

With the necessary prerequisite, the evolutionary process is discussed as follow:

\section{Initialization}

The very beginning of the genetic algorithm is to randomly generate the initial population and use a set of variables or chromosomes to represent the potential solution of an optimization problem. The size of population depends on the character of the problem and the representing process is called encoding. Encoding is very important since inappropriate encoding may cause the loss or flaws of vital original information. When 
decoded to real values, the variables need to be located within their confinement, otherwise, it is meaningless.

There are many kinds of encoding method and the most popular two are binary code and gray code. It is not hard to guess that the idea of binary string encoding is to use 0 and 1 to code the solution following the binary rule. Obviously, it is very simple and efficient. However, gray coding is more preferred since it gets over the issue of hidden representative bias. The Hamming distance which is defined as the number of positions at which the corresponding symbols are different between two strings of equal length is always constant in adjacent unit under gray coding. The comparison between binary coding and gray coding are listed in Table 1 as:

Table 1. Comparison between binary coding and gray coding

\begin{tabular}{|l|c|c|c|c|c|c|c|c|}
\hline Actual value & 0 & 1 & 2 & 3 & 4 & 5 & 6 & 7 \\
\hline Binary code & 0000 & 0001 & 0010 & 0011 & 0100 & 0101 & 0110 & 0111 \\
\hline Gray code & 0000 & 0001 & 0011 & 0010 & 0110 & 0111 & 0101 & 0100 \\
\hline
\end{tabular}

As shown in Table 1, the binary coding of the actual numbers is the binary format sequence while the gray coding is under the pattern that only one bit difference occurs corresponding to adjacent real value.

\section{Selection}

After initialization step, selection follows. A proportion of the current population is selected to form the new generation. The number of times being selected are on the basis of their corresponding fitness value. The fitter solutions are to be selected with more possibility.

There are many kinds of selection pattern, such as roulette-wheel selection, truncation selection or elitist selection. Roulette wheel selection is applied in this thesis for its simplicity and efficiency. The principle of roulette wheel selection is introduced as follow. The core idea is that candidates with higher fitness value have less probability of getting eliminated than candidates with lower fitness value. The fitness value assigned to each possible solution can be expressed as: 


$$
p_{i}=\frac{f_{i}}{\sum_{j=1}^{N} f_{j}}
$$

where $N$ is the number of individuals in the population.

It is similar to a roulette wheel in the casino and that is how this selection name comes out. To have a better explanation, a figure is needed. As shown in Figure 10, a random number of blank sectors are generated lying on the outer circle. And the five individuals located on the inner circle. Their area ratios are based on their relative fitness value. The fittest individual occupies the largest part of the wheel, like No.3 individual. On the contrary, the weakest individual possesses the smallest area of wheel, like No.2 individual. So it is very likely that No.3 is selected than No.2 to breeding the next generation. Thus, the one with fitter solution is kept.

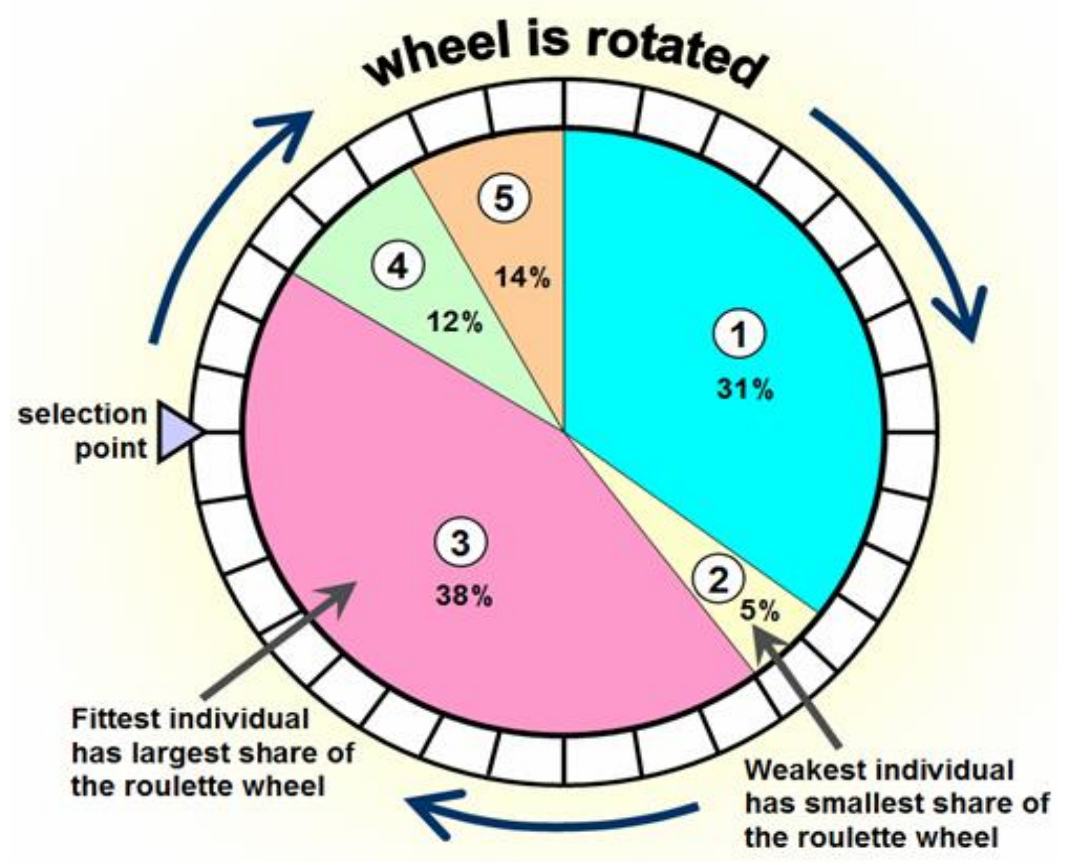

Figure 10 Explanation of roulette wheel selection [52]

\section{Reproduction}

The selected individuals need to act as parents and the offspring generated by the parent generation will absorb some characteristics from their parents. In this way, the premium aspect of potential solution is kept till the end. There are mainly two reproduction operators, namely crossover and mutation. 
Crossover: Crossover, also named recombination, is the process that aims at varying the programming of a chromosome from one generation to the next so that the fitness value of new population will be higher. There are many types of crossover techniques, such as one-point, two-point, cut \& splice and uniform crossover. A comparison example of onepoint and cut and splice is shown in Figure 11. For one-point crossover, a single point on same location of both parents' chromosome string is randomly selected. All data beyond that point is swapped between the two parents in order to generate their children. The length of chromosome is constant after crossover. For cut and splice crossover, a single point is also randomly selected but with different location on both parents. The data beyond that point is also swapped. However, the children's chromosomes have different length from their parents.

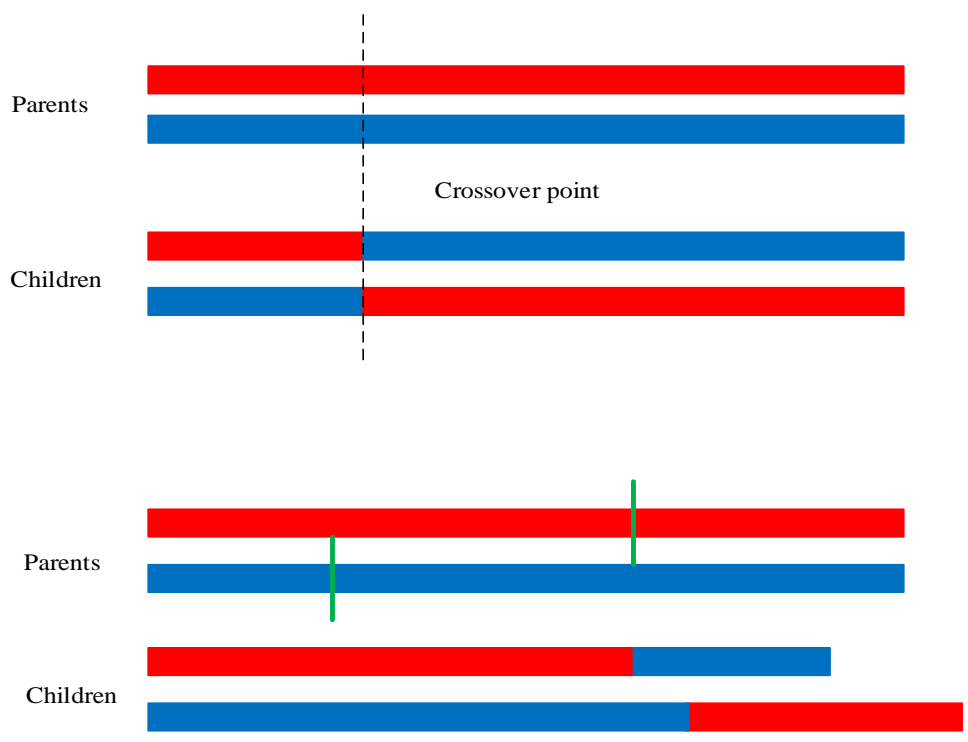

Figure 11 Example of one-point and cut \& splice crossover

Mutation: Although crossover can breed the new population, the algorithm might be trapped in a local optimum area since the genetic diversity cannot be guaranteed only by crossover operator and the chromosome might become too similar to each other. Mutation is applied to alter one or more bits in a chromosome from its initial state. Additional, only a very small percentage of the individuals are mutated, so the stability of population is ensured [55]. In mutation, the individual should be entirely different from the previous solution and it is preferable that the mutated individual locates out of the range where current population set is in. 
Many types of mutation techniques exist, for instance, bit string mutation, flip bit mutation, boundary mutation, uniform and Gaussian mutation. Among them, bit string mutation is the most classic and simple one. An example of this type of mutation is shown as:

Parents: 0000111001

Offspring: 001111001

It can be seen that the $7^{\text {th }}$ bit in parent chromosome is mutated. This mutation point is randomly selected. The probability of mutation is user defined and should be set low. If the amount of individuals mutated becomes larger, the possibility that new population converge to optimum of fitness value is mitigated. Since the higher mutation probability put the individual under the risk that the fit one might be ruined. Thus, an idea mutation probability should make the population diverse and safe at the same time.

\section{Termination}

The above selection and reproduction process are repeated until the termination condition is reached. If there is no termination step, the genetic algorithm will be trapped into an infinite loop. Commonly, these terminating conditions are used [52]:

a. A population converges to a value that satisfies minimum criteria and keeps nearby within a certain distance for a predefined iterations,

b. A fixed predefined generation number is reached,

c. Manual inspection,

d. Combinations of the above.

\section{Features of genetic algorithms}

a. Genetic algorithms are capable of dealing with not only the equation with high nonlinear properties, but also the intricate constraints. Thanks to the principle that GA tries to search the entire variable extent rather than to solve the equations proposed, it is able to calculate the fitness values, further evaluate the variables and finally obtained the optimum solution with maximum fitness value. Besides, these processes are completed without violating the constraints [56].

b. It is undoubtedly that GA is a simple approach to handle the high dimensional complex problems. It is also easily implemented and efficient. The solution provided by GA is robust over a broad search scope. 
c. Although GA may tend to locate a local optimum, it can be prevented by magnifying the mutation probability. The idea is that by doing so, the solution beyond the local optimum will also have opportunity to be taken into account.

Despite the above mentioned virtues of genetic algorithm, there still remain some limitations. It is listed as follow.

\section{Limitations of genetic algorithms}

a. When dealing with multimodal problems, the cost of evaluation of fitness function is very expensive. And it is often unaffordable to wait in real world problems such as structural optimization.

b. The termination criterion may not be very clear in every problem since GA tries to find the fitter solution. And this fitter solution is only in comparison with others.

c. GA might find its difficulty in handling the dynamic data. As the converging speed of algorithm may not be rapid enough to catch up the variation of data. Thus the optimal value obtained may not be the desirable one.

d. GA is unable to effectively solve the decision problems that there is a single right or wrong measure for fitness value. So there is no way to converge on the solution.

With the advantages and disadvantages listed, GA needs be carefully applied to fully reveal its preponderance.

\subsection{Particle Swarm Optimization}

Particle swarm optimization (PSO) is a computational method that obtains optimum solution by iteratively updating the state of candidate solution until certain criterion reached [57]. PSO is originally proposed by [58] and it is inspired by observation of the motion of the bird flocks or fish schooling. The particles which indicate the candidate solutions are moved around in the search space following a few simple rules. Their movements are guided by their own best known position as well as the global best known position. Whenever the positions with better performance are discovered, other particles will be guided by them. Unlike GAs, there is no evolution operators contained in PSO, such as crossover and mutation [57]. Since the potential candidate solution is guided by current optimum solutions, PSO is able to maintain a balance between exploration and 
exploitation. Therefore, PSO is computationally more efficient than GA in terms of speed and memory requirement. A pseudo-code of PSO is shown as:

Initialize $n$ particles $x_{i}$ and velocities $v_{i}$ within the constraint boundaries;

Compute the fitness function $f($.$) for each particles;$

Find the current best position $P_{i}$ for each particles and the global best position is denoted as $G$;

Update the particle velocities and positions using following equations:

$$
\begin{aligned}
& v_{i+1} \leftarrow \omega v_{i}+c_{1} \operatorname{rand}()\left(P_{i}-x_{i}\right)+c_{2} \operatorname{rand}()\left(G-x_{i}\right) \\
& x_{i+1} \leftarrow x_{i}+v_{i}
\end{aligned}
$$

where $\omega$ is the inertia factor and is used to balance the global and local search, $\operatorname{rand}()$ are two randomly generated coefficients followed uniform distribution $\mathrm{U}(0,1)$ and $\mathrm{c}_{1}$ and $\mathrm{c}_{2}$ are used as learning factors so that to control the impact of the social and cognitive components [59].

The termination criterion is reached, the final global best $G$ is the desired optimal solution; otherwise, return to the iteration process.

\subsection{Applications}

The methods introduced in this chapter have demonstrated good performance when applied to air conditioning systems. For instance, [60] applied multilayer perceptron and radial basis function neural network to model the automotive air conditioning system under steady state conditions and the result indicated that RBF over-perform with multilayer perceptron. [61] introduced a robust fault detection for air conditioning system based on combination of online SVM and ANN black box model. The experiment shown that the online SVM can detect accurately the air supply fan fault and damper fault with minimum usage data. [62] proposed a system identification approach for automotive air conditioning system based on genetic algorithm. The different parameters were estimated through experiment datasets and the obtained model was optimized with genetic algorithm. The performance was validated using statistical analysis based on mean squares of error which was 0.0015059 in this case. [63] presented another system identification method using particle swarm optimization. The performance of PSO was compared with that of 
recursive least squares, and the result demonstrated that the PSO offered the best model with lowest mean squares error.

\subsection{Summary}

In this chapter, the introduction of basis of artificial neural networks, the backgrounds of three different kinds of neural network, namely radial basis function, supporting vector machine and extreme learning machine, and the optimization methods including genetic algorithm and particle swarm optimization are presented in preparation for hybrid ejector air conditioning modeling and optimization. The detailed exploration of these novel neural model and optimization art in terms of air conditioning application will be discussed in the later chapters. The necessity of these intelligent techniques will be revealed later when developing the optimal model and optimization strategy for proposed hybrid systems. 


\section{Chapter 3 Performance Evaluation of Hybrid System}

\subsection{Background of three different air-conditioning systems}

\subsubsection{Background of a vapor compression system}

Vapor compression cycle is a kind of refrigeration cycle and it is the most widely used manner to generate cooling effect. It uses a circulating liquid refrigerant as the medium which absorbs and removes heat from the space to be cooled and subsequently rejects that heat elsewhere. Figure 12 depicts a typical, single-stage vapor compression system. The system is mainly consisted of four parts: a compressor, a condenser, an expansion valve and an evaporator.

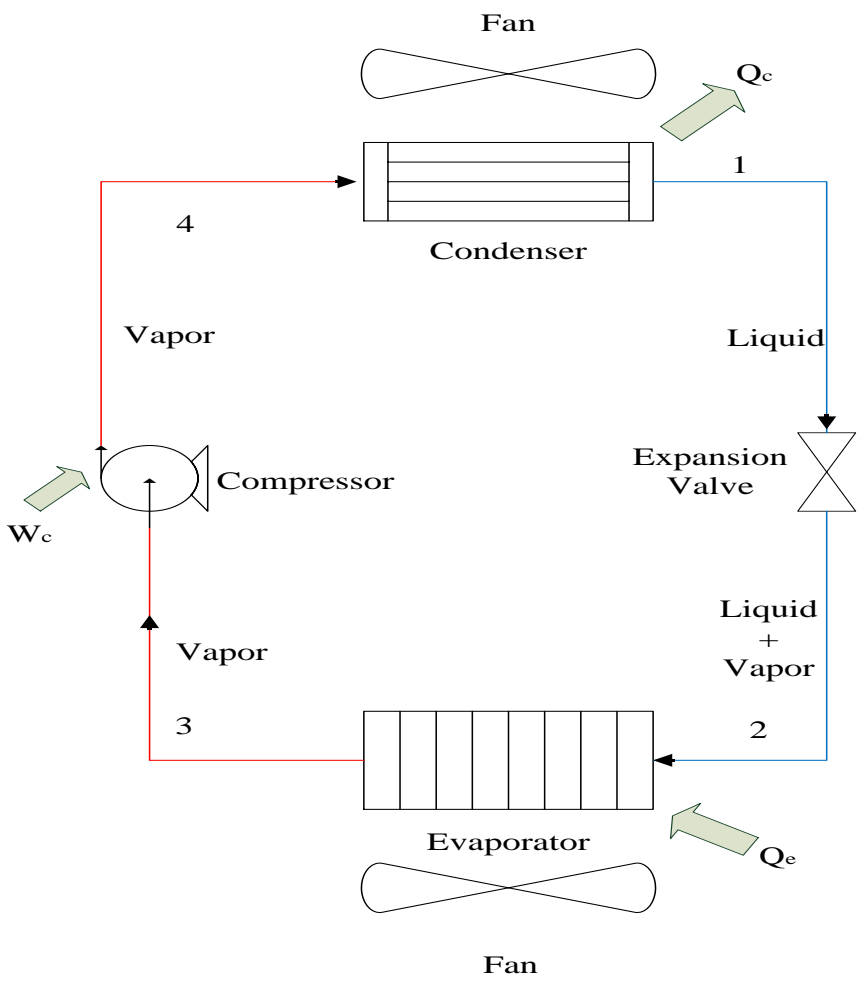

Figure 12 Vapor Compression System

The processes are elaborated as follows $[64,65]$ :

1-2: The condensed liquid refrigerant, often at a sub-cool condition, leaves the condenser and goes through the expansion valve where it undergoes an abrupt reduction in pressure. An adiabatic flash evaporation is caused by that sudden pressure reduction and part of the refrigerant becomes vapor. The auto-refrigeration effect of the adiabatic flash evaporation 
lowers the temperature of the liquid and vapor mixture refrigerant to where it is colder than the temperature of the enclosed space to be refrigerated.

2-3: The liquid and vapor mixture refrigerant now travels across the evaporator through coil or tubes. The warm air in the enclosed environment is circulated by a fan across the coil or tubes carrying the cold refrigerant. Due to the existence of temperature difference between refrigerant and warm air, heat transfer will happen, mainly convection. The liquid part of refrigerant will evaporate and the circulating air will be cooled at the same time. As a result, the temperature of the enclosed environment will be lowered to the desired level. Usually, the refrigerant at the exit of evaporator is at super-heat condition.

3-4: Then the super-heat refrigerant enters the compressor where it is compressed to a higher pressure, resulting in a higher temperature as well. Usually an adiabatic compression process is assumed because the process happens promptly.

4-1: The hot, compressed vapor goes into the condenser where it is cooled and condensed into a liquid form by flowing through a coil or tubes with cool air flowing across the coil or tubes. The working principle is similar to evaporator. The heat stored in the refrigerant is rejected to the ambient environment carrying away by the cold air. The refrigerant at the exit of condenser is often at sub-cool state.

To complete the refrigeration cycle, the liquid refrigerant from the condenser is routed back into the expansion valve again.

The thermodynamics character of the vapor compression cycle can be analyzed on a P- $h$ diagram and is shown in Figure 13. The detail explanation is following.

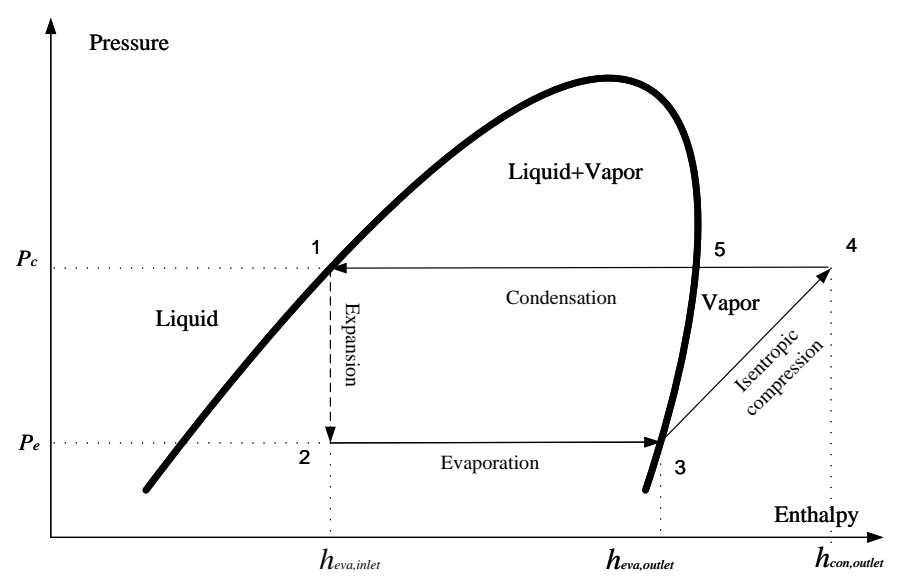

Figure 13 Ideal vapor compression cycle 
The bold curve on the diagram is called the saturation curve which separates liquid, vapor and liquid and vapor mixture regions.

In the ideal vapor compression cycle, point 1 is always at saturation liquid form. Thus it lies somewhere on the left side of saturation curve. Its location can be easily determined with given refrigerant property, either pressure or enthalpy.

Then the refrigerant goes through expansion valve where the condensing pressure is reduced to evaporating pressure. Because this process is isenthalpic, point 2 is determined by the intersection of a vertical line drawn through point 1 and a horizontal line drawn through corresponding evaporating pressure. Due to the partial vaporization of the refrigerant, point 2 is a liquid vapor mixture which lies in the mixture region.

Next, the liquid vapor mixture refrigerant is fully vaporized in the evaporator and become saturated vapor. Since vaporization process is at constant temperature and pressure, point 3 is located on the saturated vapor line where it intersects with the horizontal line drawn from point 2.

In the ideal cycle, the refrigerant properties remain constant when flowing through the suction line. Hence, process 3-4 happens within the compressor where the evaporating pressure is compressed to saturation pressure within the condenser. Since the compression process is often assumed to be isentropic, friction losses are ignored. Point 4 can be found at the intersection of constant entropy line emitted from point 3 and constant pressure line lead from point 1.

During the compression process, the vapor continually absorbs energy and the temperature of refrigerant at the outlet of compressor is higher than the saturation temperature corresponding to current pressure. This amount of super-heat must be rejected before the condensation of refrigerant can happen. Line 4-5 denotes this cooling process. Part of this process happens at the discharge line of compressor and the rest occurs in the upper portion of condenser. 4-5 is of constant pressure manner thus point 5 can be settled as the intersection of a horizontal line from point 4 and the saturated vapor curve. The enthalpy difference between points 4 and 5 represents the amount of sensible heat per unit mass of refrigerant be removed. 
Line 5-1 represents the condensation process which involves phase change of refrigerant from saturated vapor to saturated liquid within the condenser. As condensation is of constant temperature and pressure pattern, a horizontal line connects points 1 and 5 . The total amount of heat rejected from refrigerant to the ambient environment is the sum of heat quantities removed during 4-5-1.

There are many assumptions in an ideal vapor compression cycle. For instance, the refrigerant at the exit of evaporator is assumed as saturated vapor which rarely happens in the real situation. Since the cold, saturated vapor inside the evaporator will continue absorb heat from the enclosed environment. Also the vapor will absorb heat while traveling through the suction line. As a result, before it reaches the compressor, it is superheated. Another assumption is that the refrigerant at the exit of the condenser is at saturated liquid state whereas it actually is at sub-cool condition. All these variations lead to a consequence that the real refrigeration cycle is less efficient than the ideal one. Figure 14 shows the $P$ - $h$ chart of real vapor compression cycle taking into account of super-heat and sub-cool phenomenon.

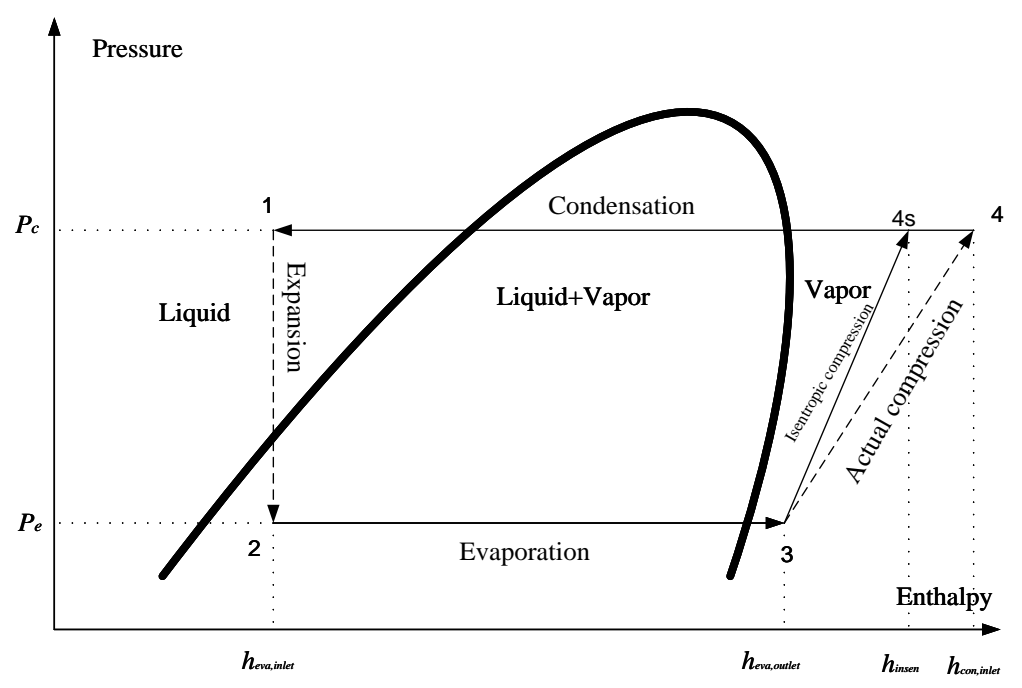

Figure 14 Real vapor compression cycle

Different from the vapor compression refrigeration system, ejector refrigeration system is shown in next section. The advantage of ejector refrigeration system over vapor compression refrigeration system will be explained. 


\subsubsection{Background of an ejector refrigeration system}

An ejector refrigeration system is shown in Figure 15 and it mainly consists of a generator, an ejector, a condenser, a pump, an expansion valve and an evaporator. The most significant difference between vapor compression system and ejector refrigeration system is that the compressor is replaced by an ejector. This substitution enables the system to utilized thermal energy. The ejector is the most crucial part of the system. The whole system performance largely depends on the behavior of ejector. Thus it is necessary to have a profound understanding about ejector.

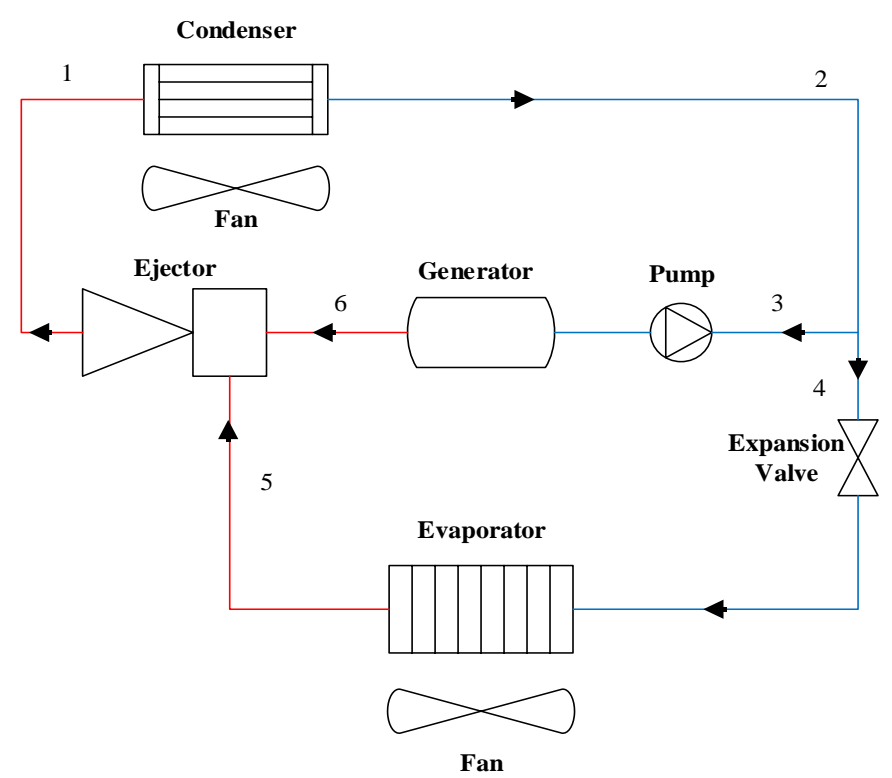

Figure 15 Ejector refrigeration system

Ejector is not a recent invented device; actually it has been invented for a long time. In 1901, [66] invented the ejector to remove air from a steam engine's condenser. Later in 1910, the first steam jet refrigeration using ejector was developed by [67] which was able to utilize low grade heat energy instead of electricity. The appearance of ejector refrigeration system was even earlier than that of the vapor compression system. Due to the low efficiency of ejector refrigeration system, it was gradually supplanted by vapor compression system. Since then, most efforts were concentrated on the improvement of vapor compression system. The development of ejector refrigeration system was almost at stagnation [68]. Nowadays, with global warming and increasing oil price, ejector refrigeration system regains people's attention. 
A schematic view of an ejector is shown in Figure 16. The corresponding process is also presented in Mollier's chart [69].

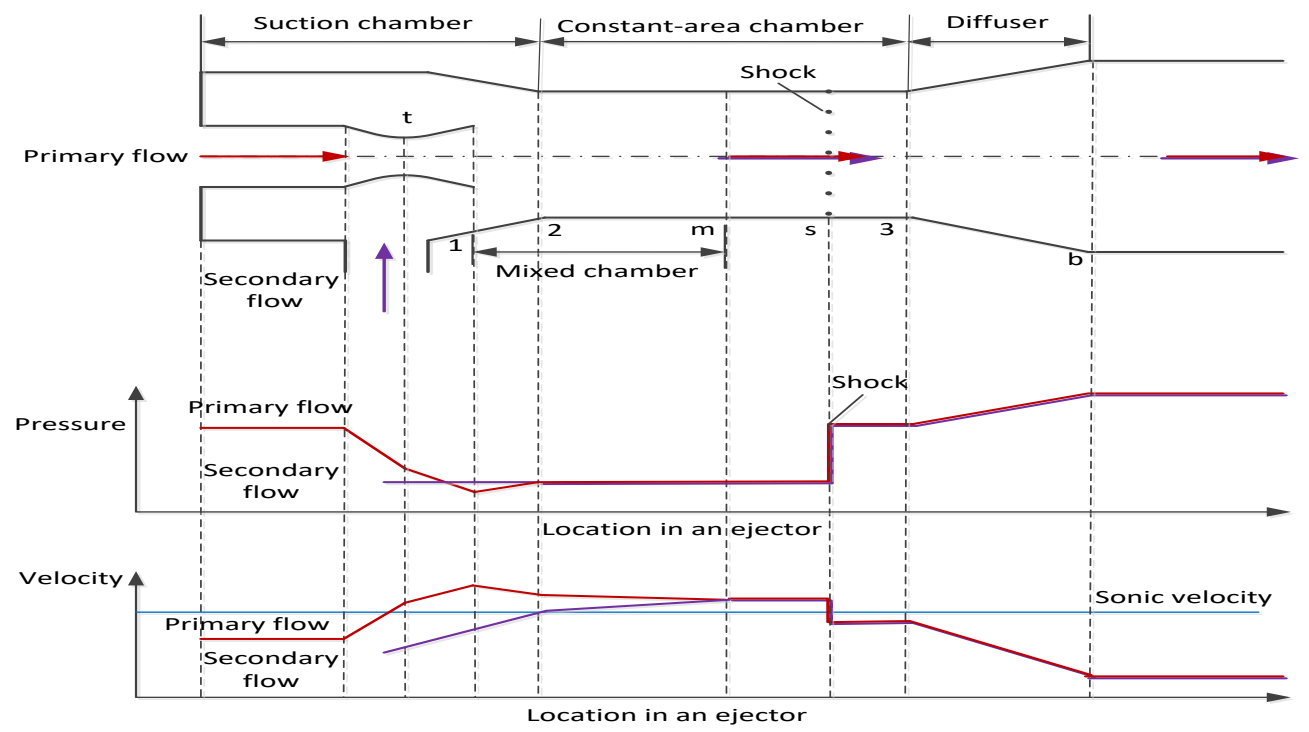

Figure 16 Schematic diagram of ejector and the relationship between pressure or velocity and location in an ejector [69]

The ejector consists of four parts: The nozzle, the suction chamber, the mixing chamber and the diffuser.

The working principle of the ejector is this [68]: The primary flow (sometimes referred as motive flow) enters the ejector with a subsonic velocity. It then goes through a converging-diverging section. During the converging part, it is accelerated to high velocity and the pressure is reduced. The velocity reaches sonic with Mach number equals to 1 at the nozzle throat and the flow chokes. When it continues passing through the diverging part, the velocity increases again and reaches supersonic. A very low pressure region (sometimes referred as vacuum region) is generated in the suction chamber due to this high velocity of the primary flow. Therefore, the secondary flow from the evaporator is entrained by this suction force. The two flows then mix, compress and will generate several shocks in the mixing chamber. Typically, there are two kinds of mixing chamber, a constant pressure type and a constant area type. Each one has its own character. The shock causes a sudden reduction in velocity and thereby an abrupt increase in pressure. As the mixture flow emerges from mixing chamber, it goes through another pressure recovery process in the diffuser. The kinetic energy is converted into potential energy. Finally, the pressure of mixture reaches the corresponding condensing pressure. 
The operation process of ejector refrigeration system can be described as follow:

1-2: The vapor refrigerant goes through the condenser with a phase change from vapor to liquid.

2-3,4: Part of the liquid refrigerant is pumped back to feed into the generator while the remaining part enters the expansion valve and undergoes a pressure decrease.

3-6: During the generator, the refrigerant is heated to saturated vapor state by the thermal energy obtained from certain source.

4-5: The expanded liquid vapor mixture then passes through the evaporator and becomes fully vapor.

6,5-1: The primary flow enters the ejector and generates a low pressure region which entrained the secondary flow. The two flows go through a series of complex process and finally leave the ejector with a corresponding condensing pressure.

Thus the cycle is complete.

The pressure-enthalpy chart of ejector refrigeration cycle is shown in Figure 17.

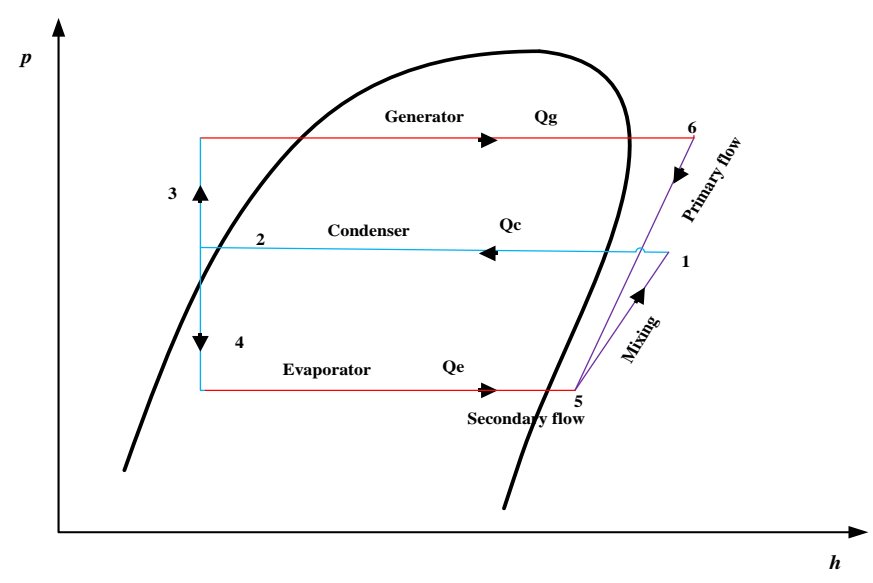

Figure $17 \mathrm{P}-h$ chart of ejector refrigeration cycle

Coefficient of performance (COP) is the most important and straight parameter to determine the performance of a system. The COP of the ejector system is the ratio of cooling capacity to power consumed by the system. Where cooling capacity means the quantity of heat been absorbed through evaporator and power consumption equals to the energy applied on generator, fan and pump. Since the power consumed by fan is little compared with that by generator and pump, it is negligible. So 


$$
C O P=\frac{Q_{e}}{W_{\text {pump }}+Q_{\text {generator }}}
$$

In order to improve the efficiency of ejector refrigeration system as well as to reduce energy consumption of vapor compression system, a hybrid ejector-based refrigeration system is introduced. The detail information will be elaborated in next section.

\subsubsection{Background of a hybrid ejector-based refrigeration system}

There are many criterions to classify researches related to the ejector-based refrigeration system. Based on the function performed by ejector, it can be divided into two types. The ejector is still used to increase the pressure of working fluid in the first type, while it acts as an expansion device in the second type. While according to the kind of energy source utilized by ejector, it can be categorized as solar-powered, gravitational, thermal, heat piped ejector refrigeration systems. Also on the basis of number of ejector applied, it can be split into single ejector, bi-ejector and multi-ejector refrigeration system [70]-[74].

In our project, a hybrid ejector-based refrigeration system is proposed when considering the advantage of both vapor compression system and ejector refrigeration system. The diagram of the hybrid ejector-based refrigeration system is shown in Figure 18. It is a combination of vapor compression system and the ejector refrigeration system. The difference between the hybrid ejector-based refrigeration system and the conventional ejector refrigeration system is the application of compressor. The objective of compressor is to compensate the low pressure value at the exit of evaporator so that the pressure difference between secondary flow and low pressure region inside the suction chamber is big enough to accomplish desired entrainment quantity. The ultimate goal is to achieve higher COP. The difference between the hybrid ejector-based refrigeration system and the vapor compression system is the application of ejector. The objective of ejector is to utilize the low grade heat energy in order to improve the performance of automobile. Since it is a combined cycle, the hybrid ejector-based refrigeration system inherits the virtue from both vapor compression system and the ejector refrigeration system. 


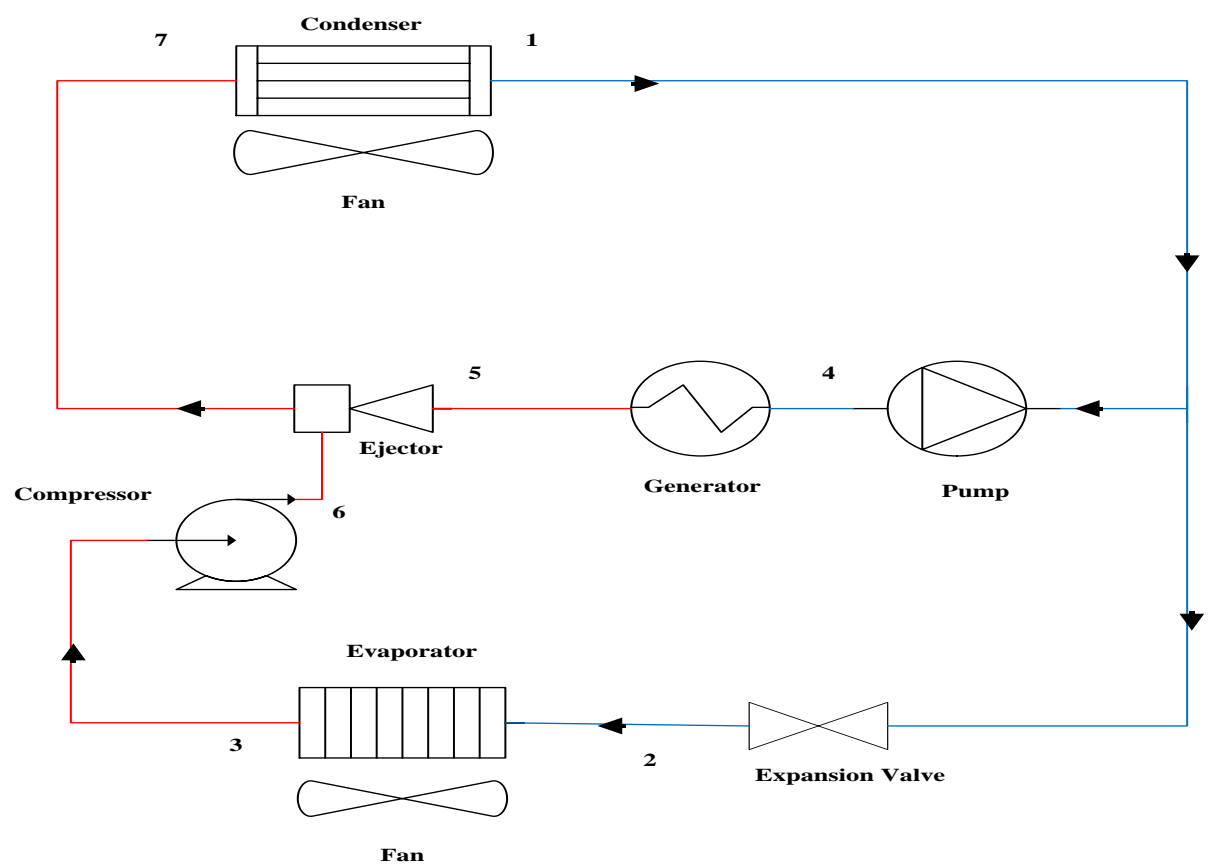

Figure 18 Hybrid ejector-based refrigeration system

The hybrid ejector-based refrigeration system is able to operate under three different modes, namely, general mode, ejector mode and hybrid mode. By switching on and off certain valve, the operation mode can be easily changed from one mode to another. Since the research about general mode and ejector mode has been conducted extensively, this project is focused on hybrid mode and the performance comparison between hybrid mode and general mode. The pressure-enthalpy chart using R-134a as refrigerant under hybrid mode is shown in Figure 19.

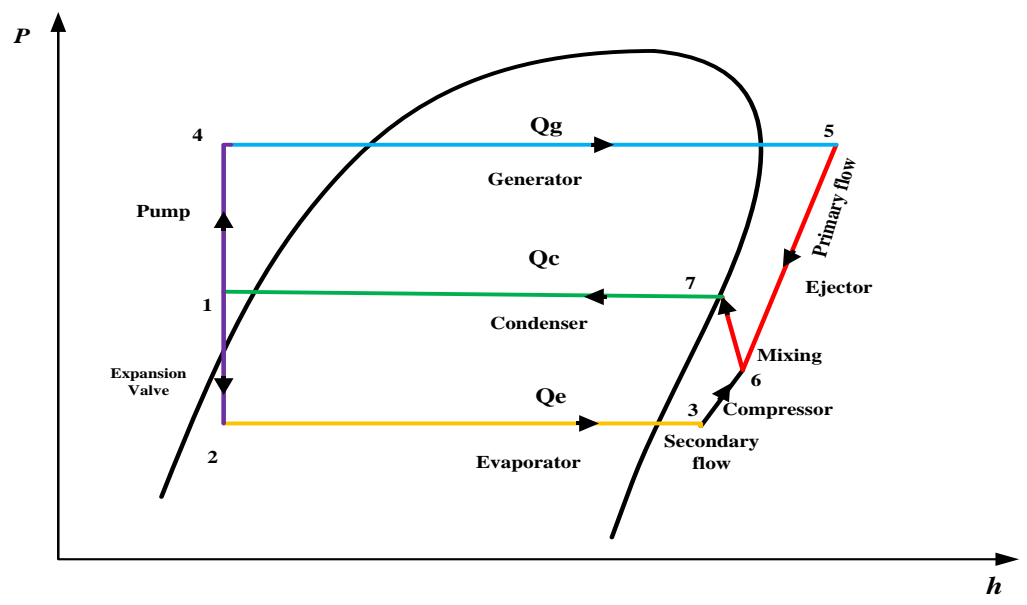

Figure 19 P- $h$ chart of hybrid mode operation 
In order to evaluate the performance of the system under hybrid mode, it is necessary to find the COP. Since COP requires the knowledge of power consumption quantity, it is inevitable to calculate the total amount of power input. There are four energy consumption components in this system, the compressor, the generator, the liquid pump and the fan of both condenser and evaporator. However, the energy being used in the generator is the waste heat, so it is not accounted for the energy consumption. Additional, the energy consumed by the fan is insignificant when compared with others', thus it is often ignored during calculation. Therefore, the COP of hybrid ejector-based refrigeration system under hybrid mode is expressed as follow:

$$
C O P=\frac{Q_{e}}{W_{\text {pump }}+W_{\text {compressor }}}
$$

Another important parameter in evaluating the performance of ejector is called the entrainment ratio which equals to the ratio of the mass flow rate of secondary flow and the mass flow rate of primary flow. It is a parameter which estimates the ability of ejector's suction effect. Usually entrainment ratio is labeled as $\omega$ and mass flow rate is marked as $m$. So the entrainment ratio can be expressed as follow:

$$
\omega=\frac{m_{s}}{m_{p}}
$$

\subsection{Experimental setup}

In order to investigate the system performance under different operation conditions, an experimental platform is set up based on previous research. A schematic diagram and photograph of experimental platform are shown in Figure 20, Figure 21 and Figure 22, respectively. The hybrid ejector-based refrigeration system has 21 major components: an inverter compressor, an evaporator, an air-cooled condenser, a receiver, an ejector, a liquid pump, an electric expansion valve, an oil tank with heater (generator), a drier, 2 liquid indicators, two flow meters and eight hand valves. 


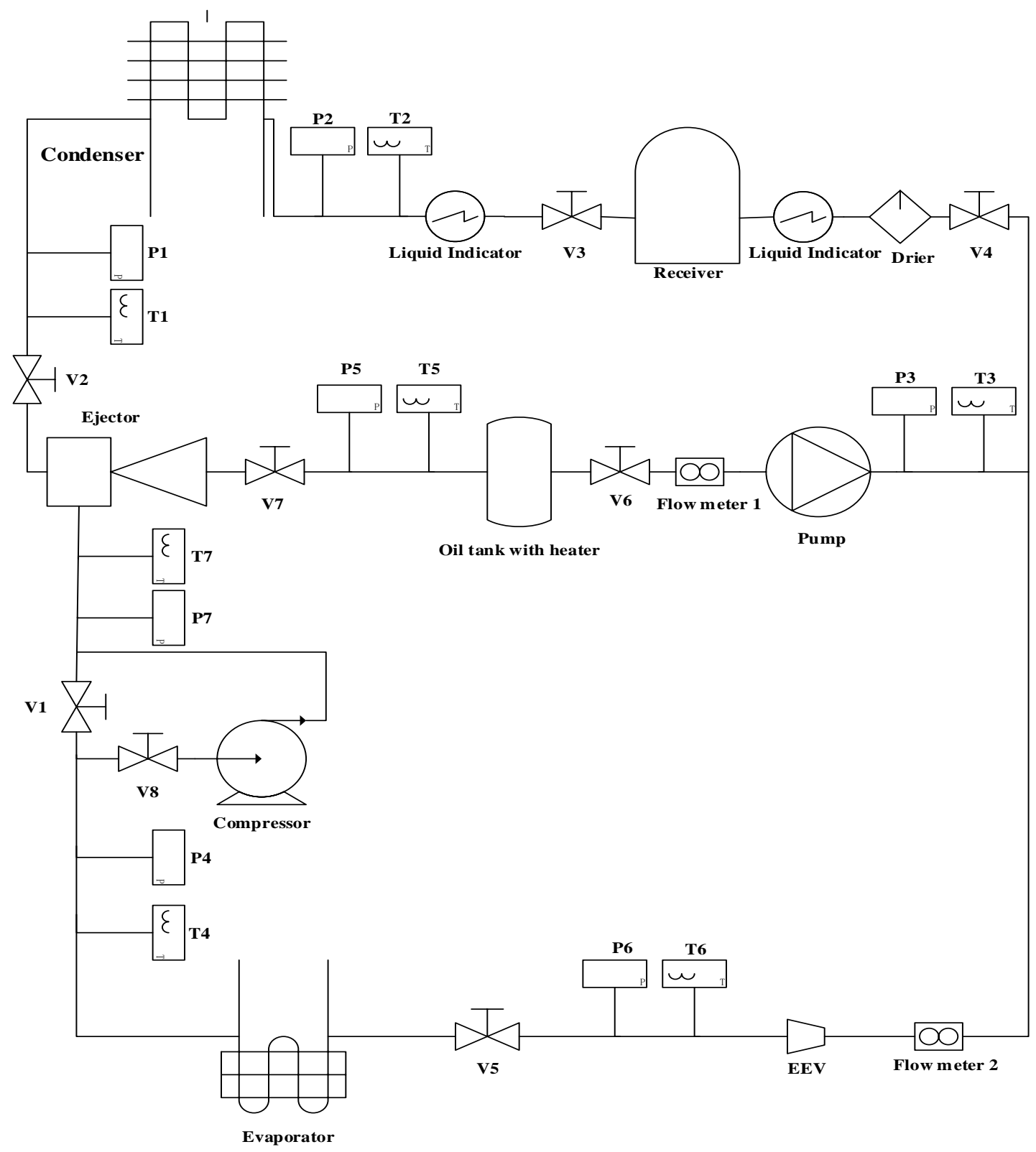

Figure 20 Schematic view of experiment platform 


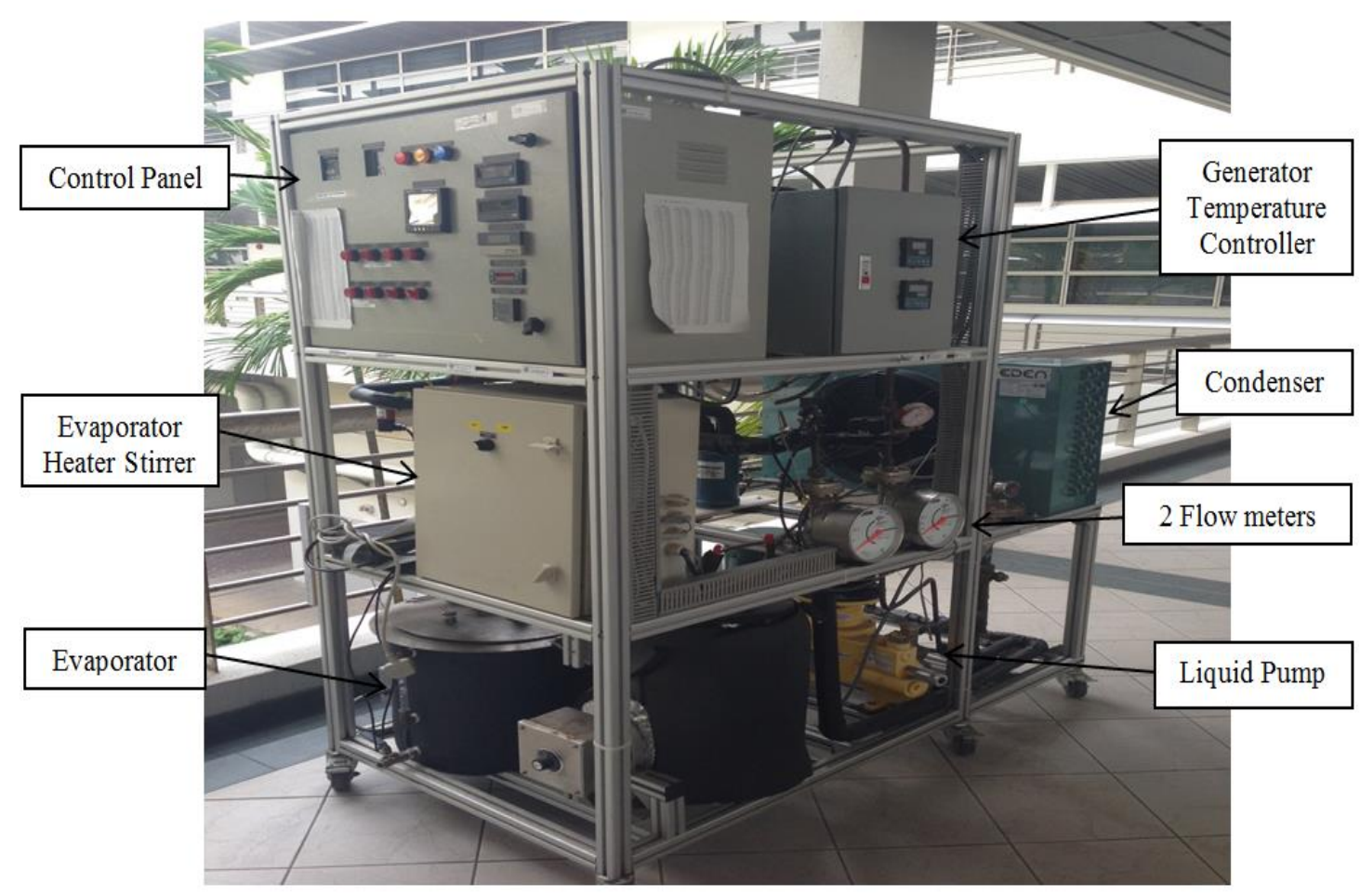

Figure 21 Frontage view of experiment platform

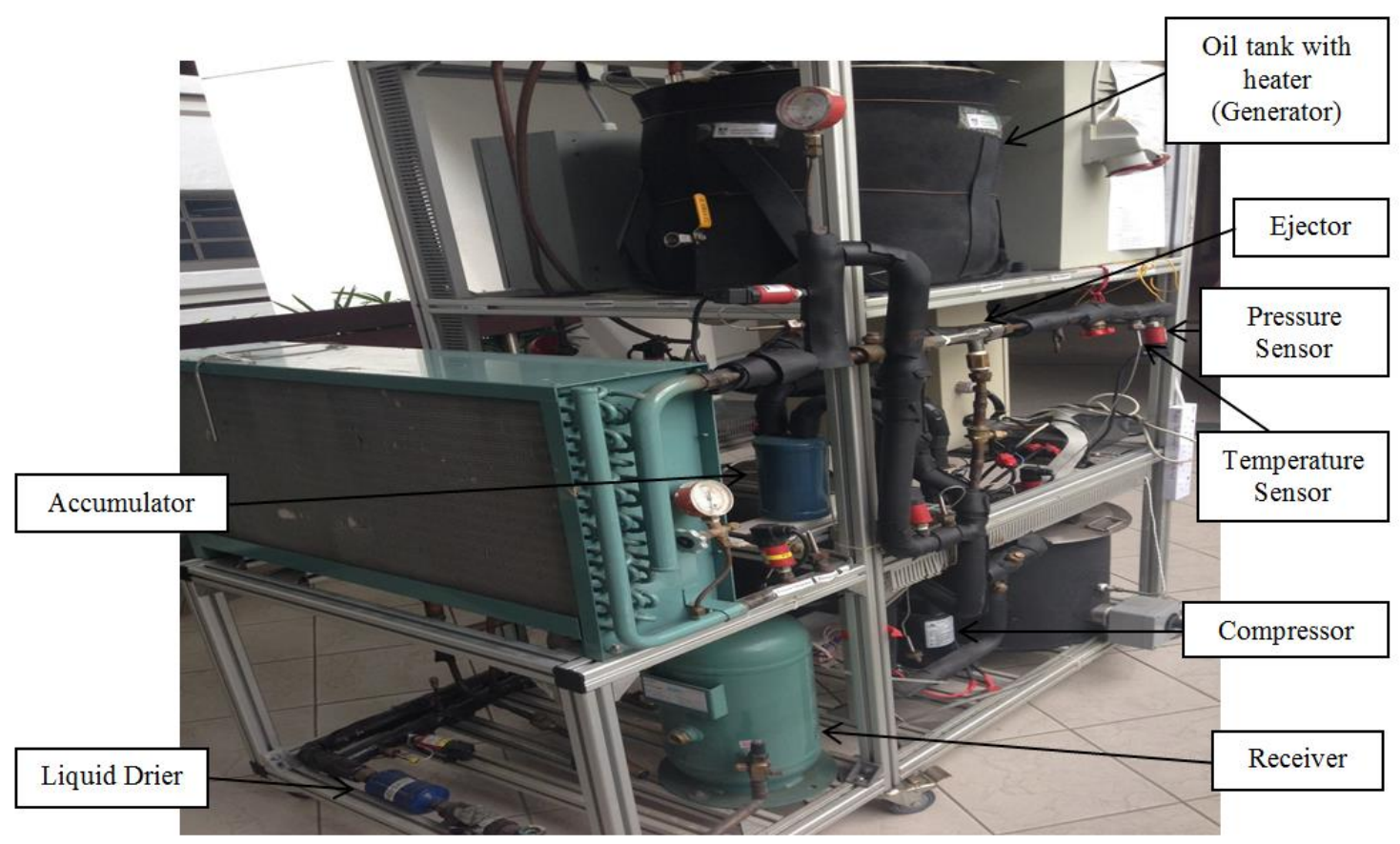

Figure 22 Rear side view of experiment platform

Except for the information of whole experiment platform, the detail operation parameters about several key components are listed as following: 
The inverter compressor is Boyard KVB135Z24, a vertical semi-hermetic compressor. It is designed to operate under DC $24 \mathrm{~V}$ and the volume of cylinder is $13.5 \mathrm{~cm}^{3}$. Its rated cooling capacity is $1850 \mathrm{~W}$ (or $6315 \mathrm{Btu} / \mathrm{H}$ ) and the permissible rpm range is from 2100 to 4500. The photograph is shown in Figure 23.

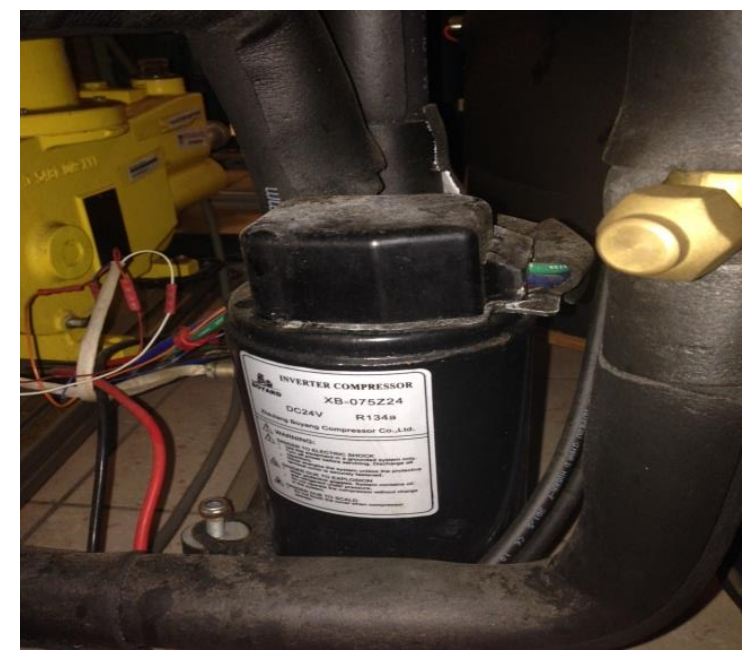

Figure 23 Photograph of compressor

The air-cooled condenser is Eden G3 matrix condenser. Its rated capacity is $15.6 \mathrm{~kW}$ and the total surface area is $28.50 \mathrm{~m}^{3} / \mathrm{h}$. The circulating fan has a capacity of $220 \mathrm{~W}$ and the volume flow rate of the air is $3600 \mathrm{~m}^{3} / \mathrm{h}$. The photograph is shown in Figure 24 .

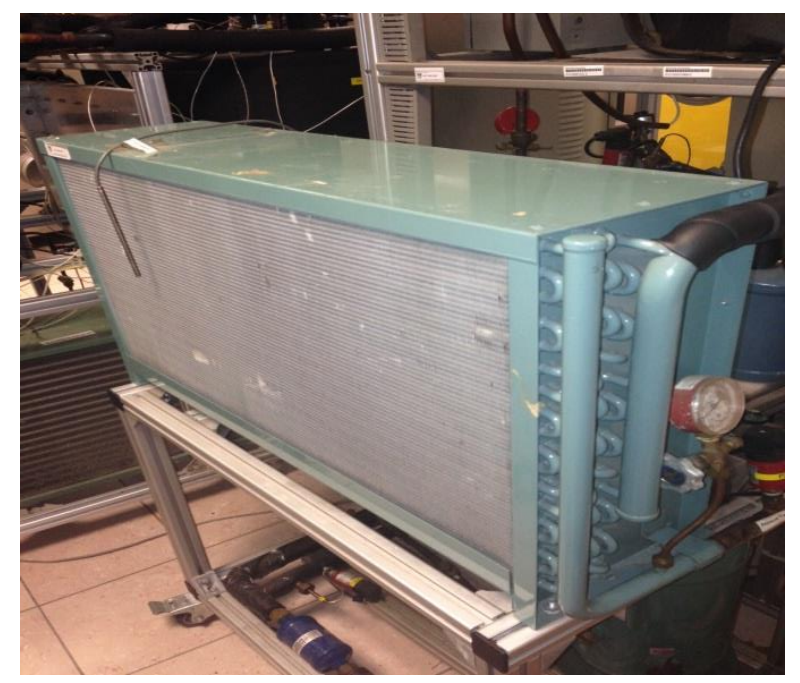

Figure 24 Photograph of condenser

The ejector is single phase type with constant mixing area. There are two ejectors with different diameter of primary nozzle exit which are used for comparison study. The designed operation conditions are: condensing temperature is between $33 \sim 35{ }^{\circ} \mathrm{C}$, 
evaporating temperature is between $7 \sim 10^{\circ} \mathrm{C}$, generating temperature is around $72^{\circ} \mathrm{C}$. The photograph is shown in Figure 25 and the AutoCAD pictures are shown in Figures 26 and 27.

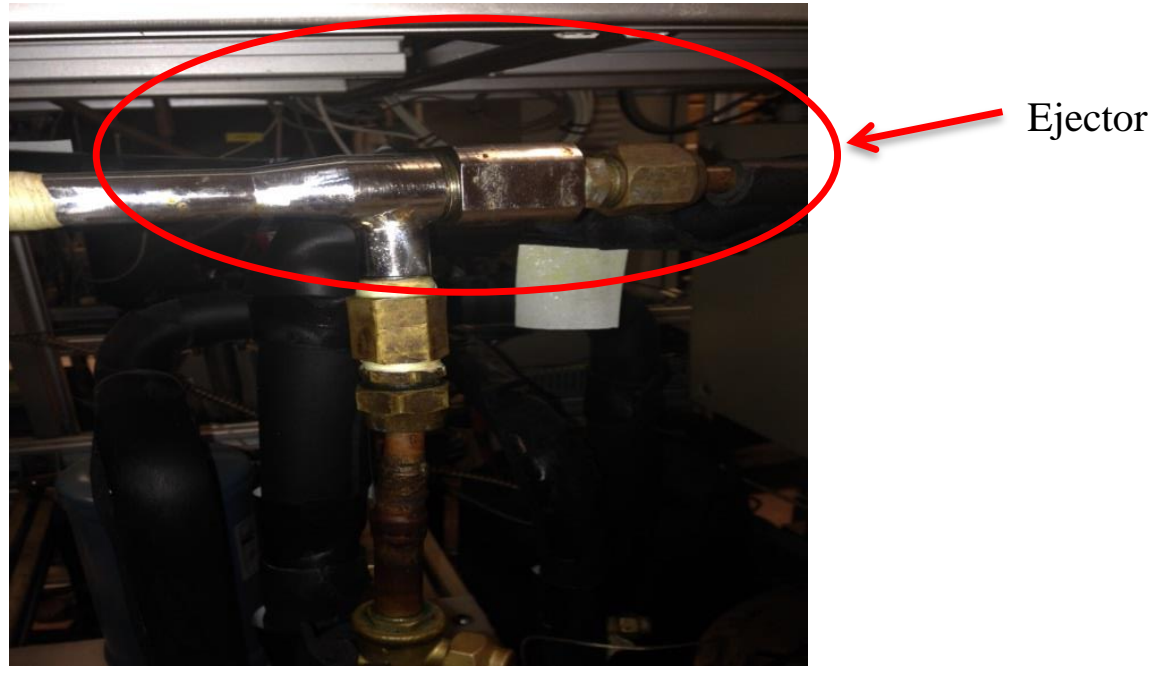

Figure 25 Photograph of ejector

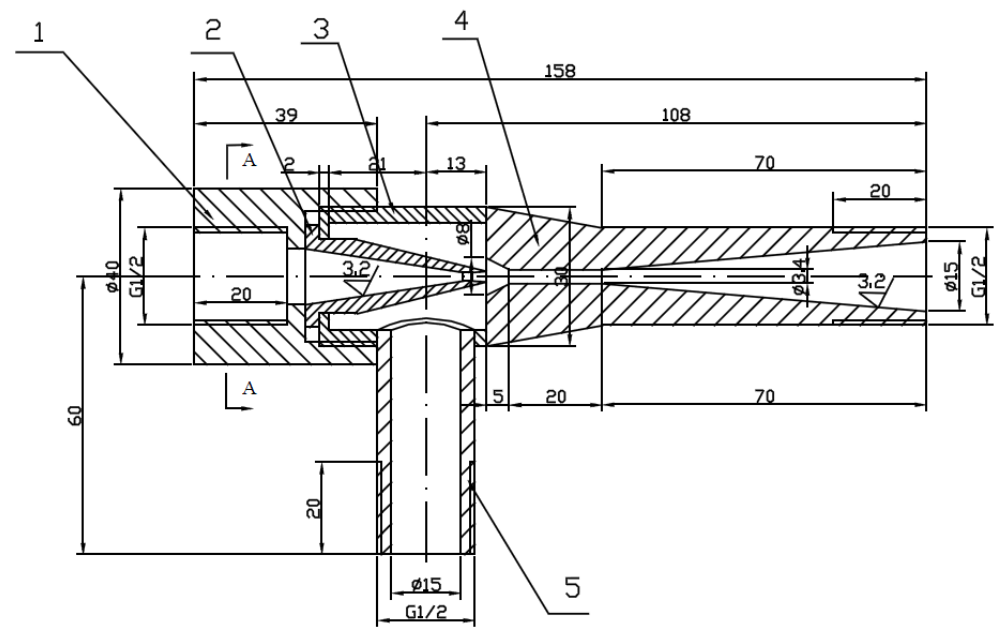

Figure 26 The detail information about ejector A 


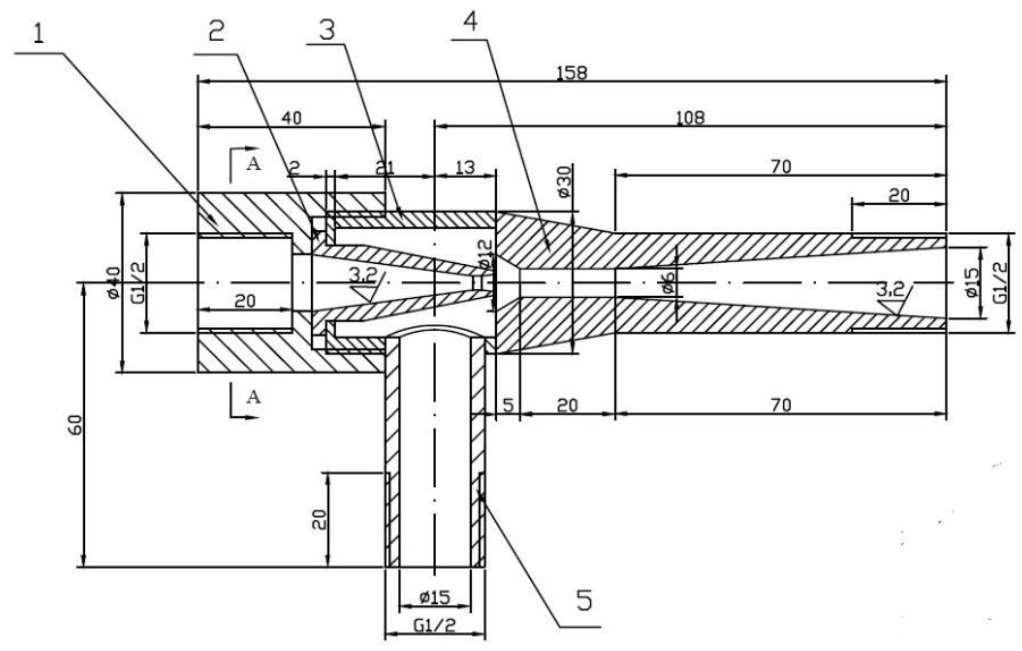

Figure 27 The detail information about ejector B

* Diameter at nozzle throat $\mathrm{D}_{\mathrm{tA}}=1.6 \mathrm{~mm}, \mathrm{D}_{\mathrm{tB}}=3 \mathrm{~mm}$.

* Diameter at nozzle exit $\mathrm{D}_{\mathrm{eA}}=2.3 \mathrm{~mm}, \mathrm{D}_{\mathrm{eB}}=3.8 \mathrm{~mm}$.

* Mixing chamber diameter $\mathrm{D}_{\mathrm{mA}}=3.4 \mathrm{~mm}, \mathrm{D}_{\mathrm{mB}}=6 \mathrm{~mm}$.

* Diffuser exit diameter $\mathrm{D}_{\mathrm{fA}}=\mathrm{D}_{\mathrm{fB}}=15 \mathrm{~mm}$.

* The distance between nozzle exit and the mixing chamber inlet $\mathrm{NXP}_{\mathrm{A}}=\mathrm{NXP}_{\mathrm{B}}=5 \mathrm{~mm}$.

Mixing chamber length $\mathrm{L}_{\mathrm{mA}}=\mathrm{L}_{\mathrm{mB}}=20 \mathrm{~mm}$.

* Diffuser length $\mathrm{L}_{\mathrm{fA}}=\mathrm{L}_{\mathrm{fB}}=70 \mathrm{~mm}$.

* Nozzle divergent section length $\mathrm{L}_{\mathrm{nA}}=4 \mathrm{~mm}, \mathrm{~L}_{\mathrm{nB}}=5 \mathrm{~mm}$.

All the parameters listed above are determined through computational fluid dynamics (CFD) simulation process (which will not be illustrated in this thesis) with the design system operation conditions. The area ratio of ejector equals to the area at mixing chamber over the area at nozzle throat. Therefore, in this case, the area ratio for ejector A is calculated by Area $_{\text {ratio }}=\frac{\pi D_{m A}^{2} / 4}{\pi D_{t A}^{2} / 4}=\frac{D_{m A}^{2}}{D_{t A}^{2}}=\frac{3.4^{2}}{1.6^{2}} \approx 4.52$, and the area ratio for ejector B is obtained via same way and equals 4 .

The EEV (electric expansion valve) is stimulated by a motor operating in step fashion which is further under the regulation of a proportion-integration-differentiation (PID) controller. It can change the opening percentage of the valve in order to adjust the mass 
flow rate of secondary flow. Every time before and after the experiment, the EEV needs to be reset. The photograph is shown in Figure 28.

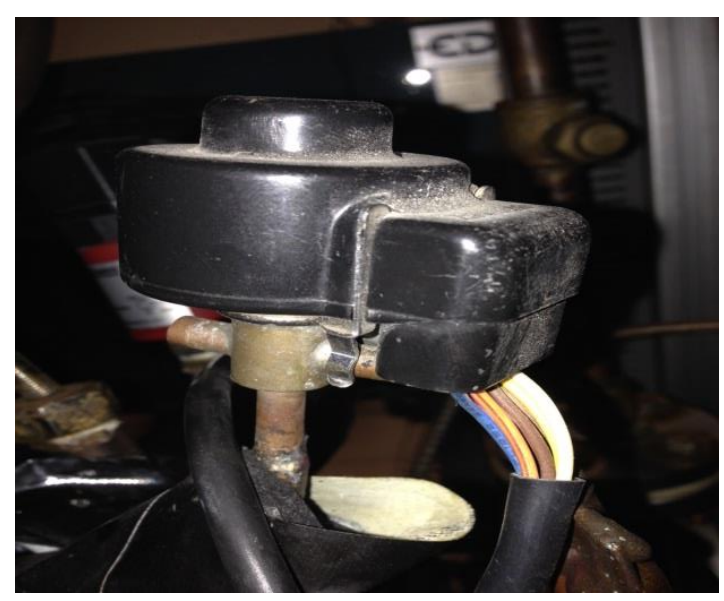

Figure 28 Photograph of EEV

The evaporator is specially designed which differs from conventional one. A heater powered by electricity is employed in the evaporator in order to maintain the operating condition constant at $32^{\circ} \mathrm{C}$. The cooling capacity means the amount of heat absorbed by refrigerant flowing through evaporator. Usually the cooling capacity equals to the enthalpy difference between evaporator inlet and outlet multiplied by the mass flow rate going through it. Since the evaporator temperature is constant, the amount of heat absorbed by the refrigerant equals to the amount of heat supplied by the electric heater. Thus cooling capacity equals to the energy consumption that generates such supplement. The photograph is shown in Figure 29.

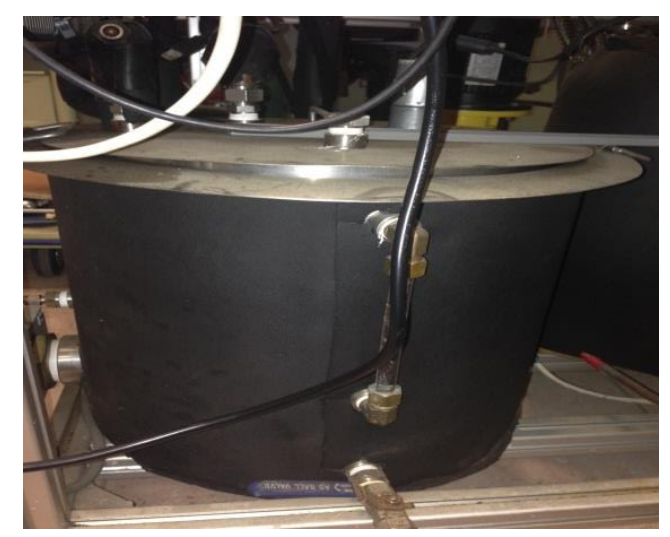

Figure 29 Photograph of evaporator

The applied generator is consisted of a vapor collection tank, a hot oil bath controlled via temperature variation and a tubular heat exchanger which is beneath the surface of the 
vapor collection tank. A variable electric heater with maximum power output reach to 8 $\mathrm{kW}$ is used to raise the temperature of oil bath. The deviation of hot oil temperature is controlled and constrained no more than $\pm 0.5^{\circ} \mathrm{C}$ through a PID type controller in the monitoring and control system. The function of generator is to simulate the waste heat energy expelled from automobile engine. The oil is heated up to $150^{\circ} \mathrm{C}$ which lies in the temperature range of low grade energy and the refrigerant leaves the generator with a temperature above $100^{\circ} \mathrm{C}$. The photograph is shown in Figure 30 .

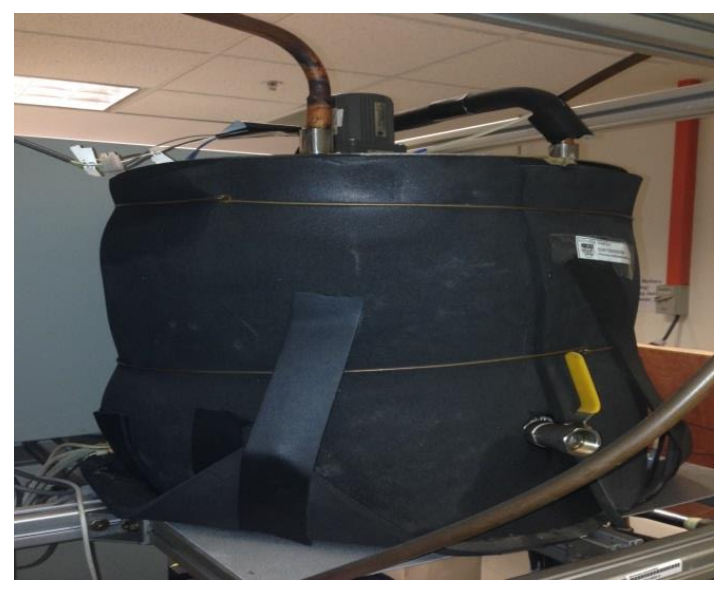

Figure 30 Photograph of generator

The liquid pump is Milton roy with a capacity of $248 \mathrm{l} / \mathrm{h}$. The rated operation pressure is 28 bar and the maximum temperature of pumped fluid is $90^{\circ} \mathrm{C}$. The power output can be adjusted by rotary knob on the side. In order to avoid the potential damage caused by cavitation, the liquid pump is placed and mounted at the bottom along with a liquid receiver tank of large size. The photograph is shown in Figure 31.

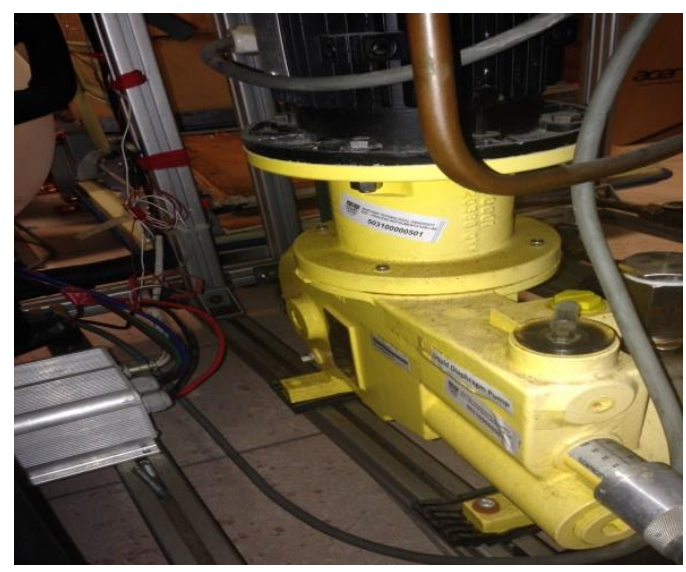

Figure 31 Photograph of liquid pump 
The hand valves in the experimental system are used for operation mode transition and system diagnosis such as leakage diagnosis. Switch on valve V2, V3, V4, V5, V6, V7 and V8 while switch off V1, the system will operate in hybrid mode. Switch on valve V2, V3, $\mathrm{V} 4, \mathrm{~V} 5, \mathrm{~V} 8$ and switch off valve V1, V6, V7, the system is under vapor compression mode (or general mode). Switch on valve V1, V2, V3, V4, V5, V6 and V7 while keep V8 off, the system will run in ejector mode which will not be discussed here. With this setup, we can easily switch between these modes and make a better performance comparison test. In the hybrid ejector-based refrigeration system, all the equipment, no matter high temperature or low temperature, is well insulated by foam rubber to prevent any heat losses.

All the data acquisitions and control algorithms are developed in National Instruments LabVIEW 2010 [75].

Several types of sensors are installed in the specific position of system to record the needed information:

* Pressure transmitters with error of $0.5 \%$ of full scales (0 60bar).

* Temperature sensors of PT1000 platinum resistance with error of $\pm 0.3^{\circ} \mathrm{C}$.

* Flow meters are calibrated with error of $\pm 1.6 \%$.

* Digital multi-meter to measure the current flowing through compressor

The refrigerant applied in the experiment is R134a as it is widely used and possesses satisfying properties. Also it has insignificant ozone depletion potential and a relative lower global warming potential feature [76].

The experimental plant was usually brought to steady state condition within 40mins after the inputs were changed. Data collection process was begun as soon as the system stabilized. The refrigerant enthalpies are determined from measured temperature and pressure values according to NIST database. The total expanded uncertainties for the airside heat transfer rate measurements are $\pm 1.5 \%$. The total expanded uncertainties for the refrigerant side heat transfer rate measurements are $\pm 3.5 \%$ for the condenser and $\pm 5 \%$ for the generator, with the biggest contributor being the uncertainties in the thermodynamic property estimation calculations. 
The design operating conditions of the system are: condensing pressure 9bar, evaporating pressure 3.5 bar and generating pressure 22 bar. The design cooling capacity is $1.5 \mathrm{~kW}$. The experiment conditions are listed in Table 2. Particularly, the tests were carried out from $9^{\text {th }}$ Aug 2013 to $2^{\text {nd }}$ May 2014 on every weekday 2pm in Singapore and the test duration was 3 hours per day.

Table 2. Operating conditions of the system

\begin{tabular}{|c|c|}
\hline Operating Condition & Value \\
\hline Ambient temperature $\left({ }^{\circ} \mathrm{C}\right)$ & $33 \sim 34$ \\
\hline Condensing pressure (bar) & $8 \sim 12$ \\
\hline Evaporating pressure (bar) & $2.5 \sim 4.5$ \\
\hline Generating pressure (bar) & $10 \sim 25$ \\
\hline Compressor frequency $(\mathrm{Hz})$ & $30 \sim 50$ \\
\hline EEV opening ratio $(\omega)$ & $30 \% \sim 60 \%$ \\
\hline
\end{tabular}

\subsection{Experiment results and analysis}

The major objective of this experiment is to investigate the effect of condenser, evaporator and generator pressure on the system performance with ejectors of different mixing chamber diameter. The controlling variables method is applied that means when investigating one particular parameter, the others remain constant. For instance, when investigating the back pressure effect on entrainment ratio, both generating pressure and evaporating pressure are kept constant. Also, the comparison between conventional vapor compression cycle and proposed hybrid cycle is of our interest. The operating conditions for two cycles are the same, only the structure difference is investigated. In order to mitigate the influence of random error, each experimental point is the average value of 50 experiment data. Moreover, based on accuracy of experimental apparatus and principle of error transfer, the relative error for entrainment ratio is $6.7 \%$, for cooling capacity is $10.1 \%$ and for COP is $13.3 \%$ [77]. 


\subsubsection{Back pressure effect on entrainment ratio}

The condensing pressure (back pressure) is closely related to entrainment ratio and is affected by ambient condition which is impossible to adjust compared with evaporating and generating pressures.

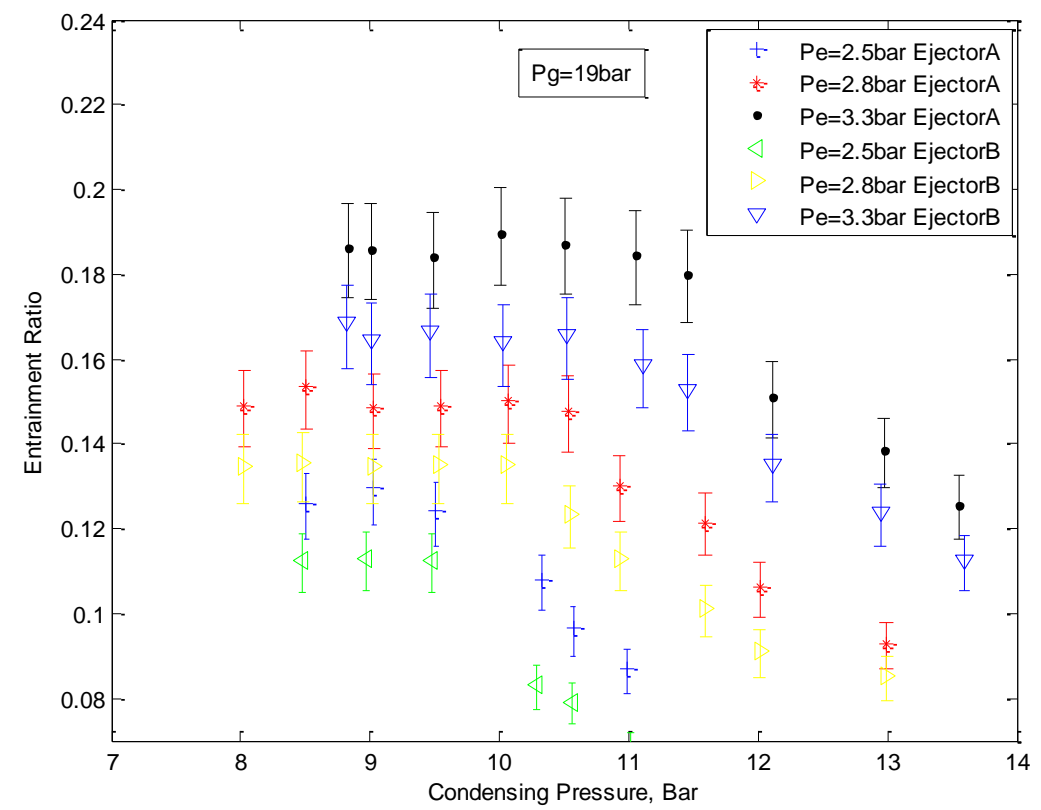

Figure 32 Variation of entrainment ratio with condensing pressure under constant generating pressure

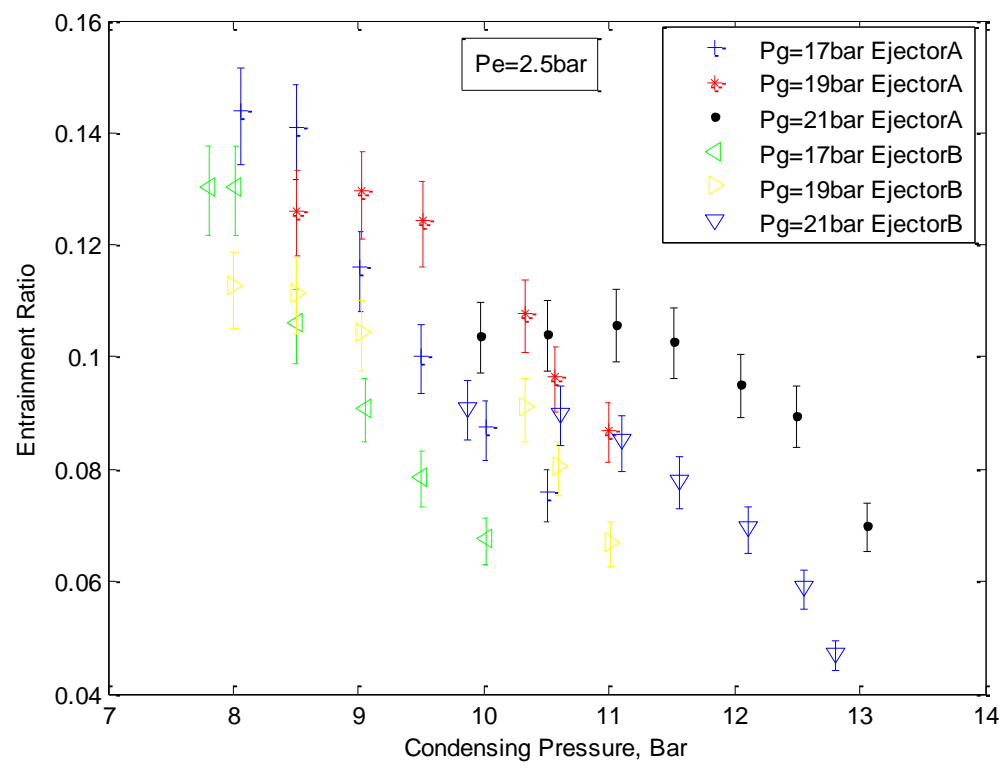

Figure 33 Variation of entrainment ratio with condensing pressure under constant evaporating pressure 
The experiments are conducted under different conditions so that the relationship between critical back pressure and evaporating (or generating) pressure can be obtained. In order to more intuitively reveal the variation trend of entrainment ratio under different operation conditions, experimental points are connected with lines. Figure 32 shows variation of entrainment ratio with condensing pressure under constant generating pressure, it can be seen that 1) under constant evaporating $\left(\mathrm{P}_{\mathrm{e}}\right)$ and generating pressure $\left(\mathrm{P}_{\mathrm{g}}\right)$, entrainment ratio remains constant before condensing pressure increases to a certain value, 2) as condensing pressure increases, the entrainment ratio drop sharply. The reason for this critical condensing pressure is that when condensing pressure $\left(\mathrm{P}_{c}\right)$ is lower than critical pressure, the mixed flow is choked within mixing chamber. Therefore, the mass flow rate of secondary flow is independent from condensing pressure. Only the increase of evaporating pressure can raise the secondary flow rate. That is why there is a horizontal part at left side of the line. When condensing pressure is higher than critical pressure, the mixed flow is no longer choked and the secondary mass flow varies with condensing pressure. The higher the condensing pressure is, the more resistance there will be at the outlet of ejector. The pressure difference between ejector inlet and outlet that generates motive force is reduced. So the entrainment ratio decreases sharply as a result. Moreover, continually increasing condensing pressure will cause reverse flow circumstance in ejector which should be highly prohibited. Also from Figure 32, it can be found that when evaporating pressure increases, both entrainment ratio and critical pressure value increase. Since the increment of evaporator pressure leads to the increase of pressure difference between secondary inlet and vacuum region, more secondary flow will be sucked into ejector due to this larger pressure difference. Thus the entrainment ratio increases with increase of evaporator pressure. Besides, the evaporator pressure augment results in larger momentum that is capable of dealing with higher condensing pressure. Hence, when evaporator pressure increases, the critical pressure value will increase that offers a wider and safer operation range for condenser. So the increases of evaporating pressure will result in a better performance of ejector with sacrifice of evaporator operation range.

The variation of entrainment ratio with the condensing pressure under constant evaporating pressure is illustrated in Figure 33. It is observed that 1) under constant evaporating pressure, increasing generating pressure causes entrainment ratio to decrease 
and 2) critical pressure value will increase with increment of generating pressure. The reason for this phenomenon is obvious that when generating pressure increases, the primary mass flow rate will increase. Since the diameter of mixing chamber is fixed and the mixed flow is choked within mixing chamber when condensing pressure lower than critical value, an increment of primary flow rate will cause secondary flow rate to decrease which leads to a reduction of entrainment ratio. The numerator which represents secondary flow rate decreases and the denominator which represents primary flow increases, together the entrainment ratio decreases. As secondary flow goes through evaporator and related to cooling capacity, this also leads to a decrease of cooling capacity and COP. However, a higher generating pressure will causes the momentum of the mixed flow to increase. Thus, the critical condenser pressure is increased. Higher generating pressure requires more power from liquid pump or higher temperature produced by generator heater. In terms of system efficiency, it is beneficial to have the system operated under lower generating pressure.

Both Figure 32 and Figure 33 indicate that the entrainment ratio of ejector $\mathrm{A}$ is higher than that of ejector B under same operating conditions. The reason for this performance is that the mixing chamber diameter of ejector $\mathrm{B}$ is $6 \mathrm{~mm}$ which is $76.4 \%$ larger than $3.4 \mathrm{~mm}$ of ejector $\mathrm{A}$ while the nozzle throat diameter of ejector $\mathrm{B}$ is $3 \mathrm{~mm}$ which is $87.5 \%$ bigger than $1.6 \mathrm{~mm}$ of ejector A. So under the same operating conditions, the ejector B ought to be able to entrain more secondary flow due to larger nozzle throat, however, since the mixing chamber diameter of ejector B is only $76.4 \%$ increase from $\mathrm{A}$, this will limit the mass flow rate of secondary flow. As a result, both primary flow and secondary flow will increase when applying ejector B, the increment amount of primary flow is higher than that of secondary flow which leads to decrease of entrainment ratio.

For better performance, the ejector with higher entrainment ratio is desired. Thus, ejector $\mathrm{A}$ is chosen to conduct the following experiments.

\subsubsection{Primary and secondary pressure effect on entrainment ratio}

The evaporating pressure on system performance is tested for both entrainment ratio and COP. Figure 34 depicts how the entrainment ratio varies with evaporating pressure. 
From the figure, it can be concluded that for the constant condensing and generating pressures, the entrainment ratio increases along with the evaporating pressure. With the increase of evaporating pressure, the pressure difference between ejector secondary inlet and vacuum region increases, thus a small amount of primary motive flow is sufficient to generate enough suction force which entrains the secondary flow into ejector. Therefore, at higher evaporating pressure value, the entrainment ratio is larger. On the other hand, an increase of generating pressure will cause the entrainment ratio to decrease. Similar trend can be observed from variation of COP with evaporating pressure which will be shown later.

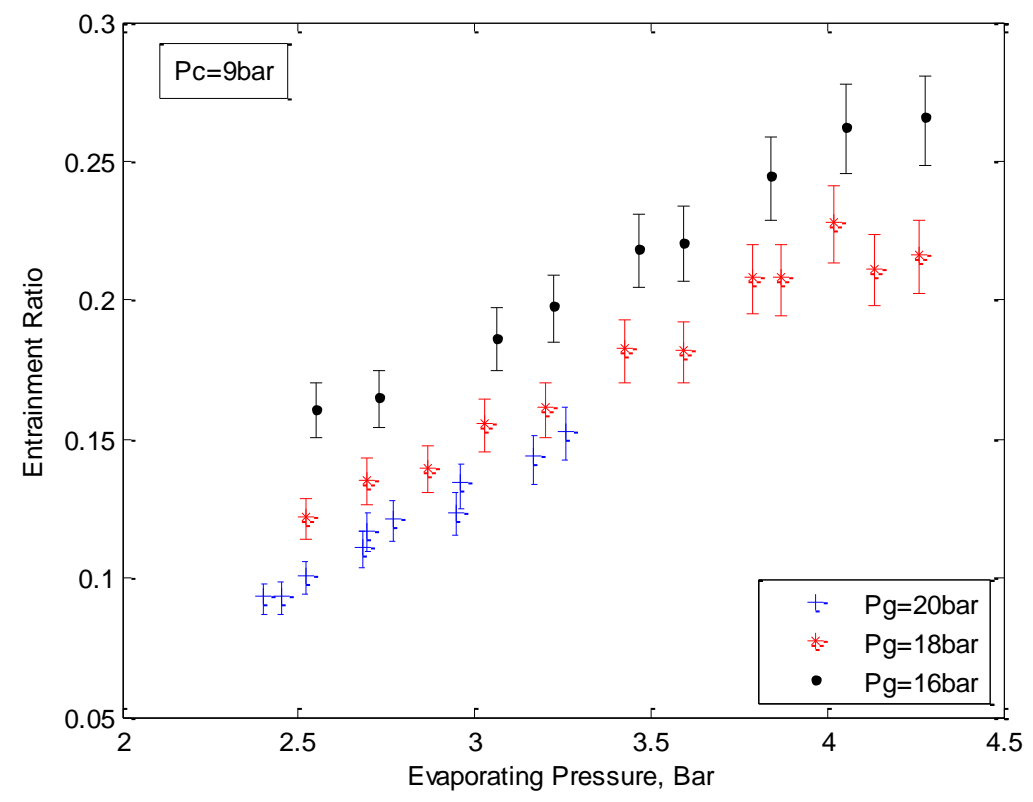

Figure 34 Variation of entrainment ratio with evaporating pressure under constant condensing pressure

In order to investigate the relationship between system performance and generating pressure, the experiment is conducted under following conditions: the $P_{c}$ is constant at 9 bar, the $\mathrm{P}_{\mathrm{e}}$ is constant at 3 and 4 bar while the $\mathrm{P}_{\mathrm{g}}$ is varied from 10 23 bar and the system is operating under hybrid mode. 


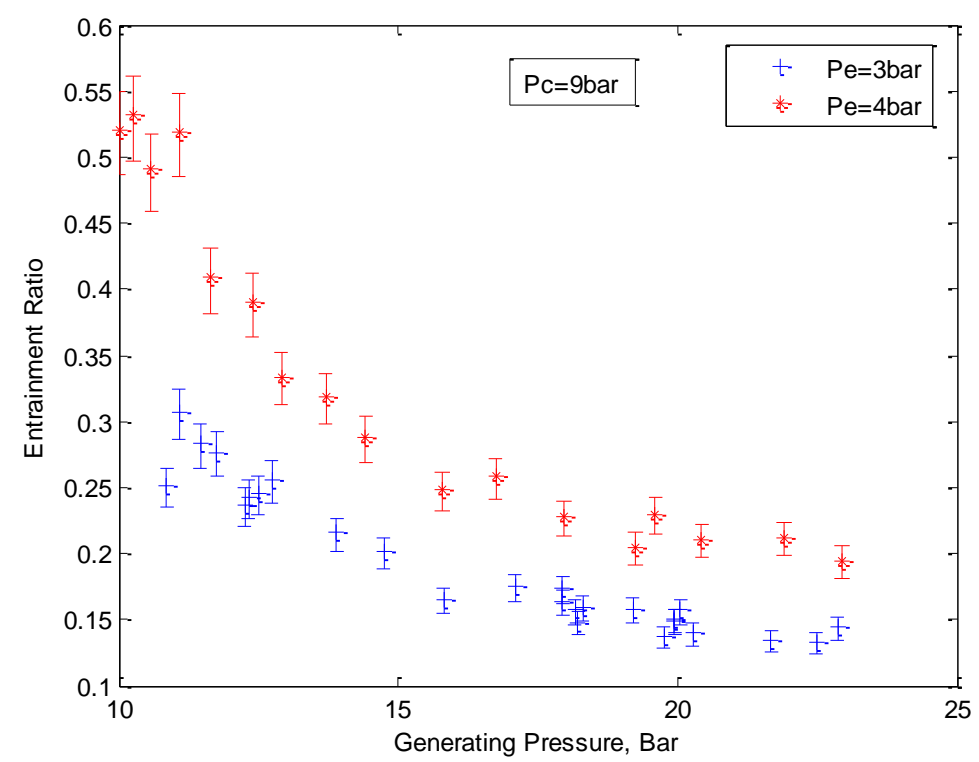

Figure 35 Variation of entrainment ratio with generating pressure under constant condensing pressure The variation of entrainment ratio with generating pressure under constant condensing pressure is illustrated in Figure 35. It can be seen that the entrainment ratio under hybrid mode is decreasing along with the increasing of generating pressure. Since under hybrid mode, compressor helps compensate suction effect even when generating pressure is not high enough. Although the entrainment ratio seems to be quite satisfying, it is due to the small primary mass flow rate, the actual cooling capacity is not at its optimum. As generating pressure keeps increasing, both the primary mass flow rate and the suction effect generated by ejector are increasing proportionally; this will help mitigate the compressor loads which in turn reduce the power consumption of compressor.

\subsubsection{Different pressure effect on COP}

Since COP is an important parameter to evaluate the system performance and also in order to improve the efficiency of the system, the influence of evaporating and generating pressure on COP is studied. The condensing pressure is not studied because its adjustment is not practical as previous two pressures in real application.

From the experiment data, it can be seen from Figure 36 that COP increases with the increment of evaporating pressure under all three generating pressures. From previous results, it is known that increase of evaporator pressure leads to increase of entrainment ratio and finally results in cooling capacity augment. Also, under constant generating and 
condensing pressure, a higher evaporating pressure is able to be entrained by a lower generating pressure which means higher evaporating pressure requires lesser power consumption of liquid pump or generator heater. This will together help improve the COP of the system. In order to achieve higher COP value, the system should be run at higher evaporating pressure condition. However, in real application, a lower evaporating temperature, which also means lower evaporating pressure, is desired. So it has to be a balance between achieving higher COP and desired evaporating temperature.

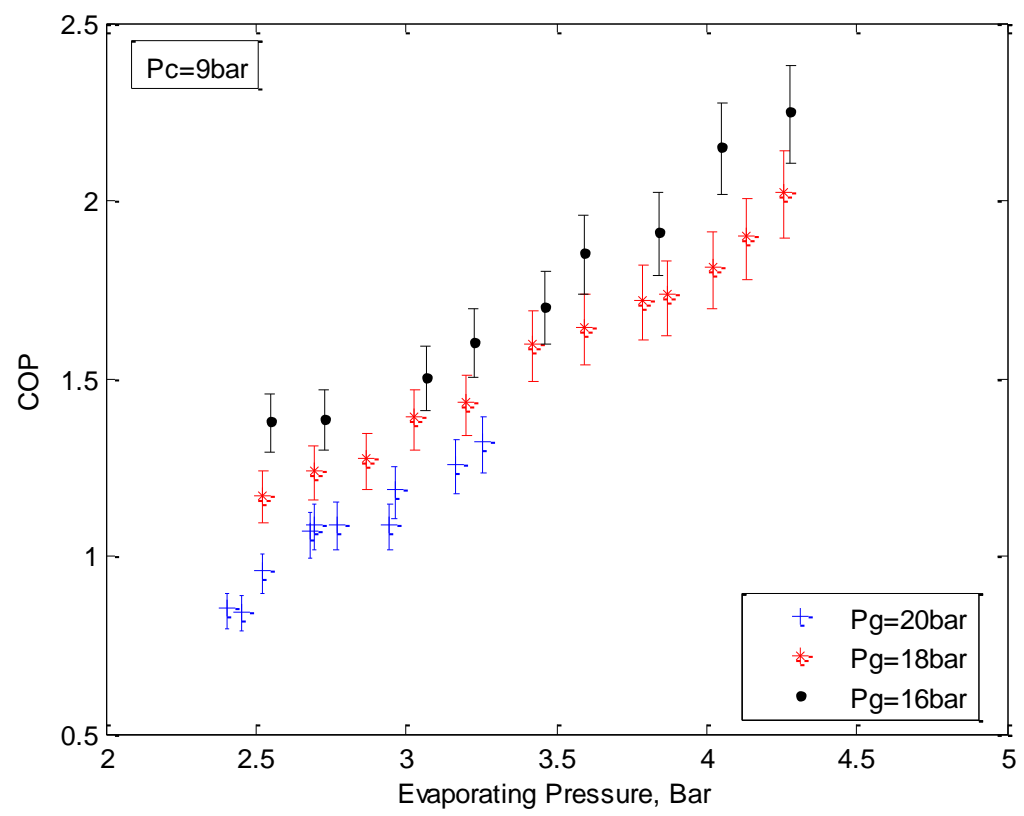

Figure 36 Variation of COP with evaporating pressure under constant condensing pressure

Additionally, the variation of COP with generating pressure is investigated in Figure 37. Under hybrid mode, for given evaporating and condensing pressure, COP is increasing with the increment of generating pressure. Although the entrainment ratio is decreasing with the increment of generating pressure, it does not mean that COP will follow the same pattern. At higher generating pressure, the mass flow rate of primary flow is higher; the suction force generated by primary flow is large enough to achieve sufficient entrain effect under relative lower secondary pressure condition. Thus the compress ratio decreases which leads to a reduction in compressor power consumption. If more exhaust heat is collected and recovered, the generating pressure is higher which results in a higher COP. 


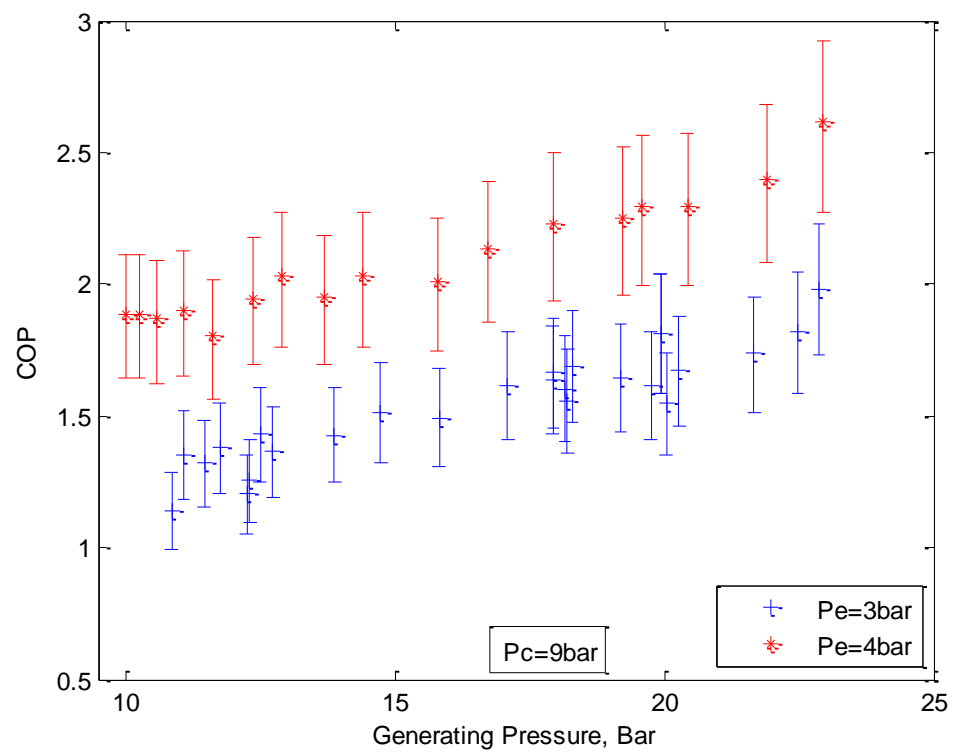

Figure 37 Variation of COP with generating pressure under constant condensing pressure

\subsubsection{Performance comparison between two modes}

The comparison of cooling capacity, power consumption and COP between general mode and hybrid mode are illustrated in Figures 38, 39 and 40, respectively. From the three figures, it can be seen that the application of ejector in hybrid refrigeration cycle (hybrid mode) can indeed improve the system performance over conventional vapor compression cycle (general mode). From COP equations of both general mode and hybrid mode, it can be found that there are two more power consumed components (liquid pump and generator heater) within hybrid mode. However, the power consumption of hybrid mode is lower than that of general mode. The reason is that the employment of passive device like ejector which utilizes the waste heat energy to generator suction force shares part of the responsibility of the compressor, particularly, the operation principle of mixing chamber in some extent are similar to a compressor which reduces the energy consumption of mechanical compressor in return. This reduced amount of energy is larger than the power consumption which liquid pump and heater bring along. Thus, the total power consumption of hybrid mode is lower than that of general mode. Additional, the use of ejector enhances the entrain effect of secondary flow that related to cooling capacity. Therefore, the cooling capacity of hybrid mode is higher than that of general mode. All these factors together contribute to the COP improvement of hybrid 
refrigeration system. Given a scrutiny towards the result, it can be found that the cooling capacity is averagely $27.3 \%$ higher under hybrid mode than that of general mode; the power consumption is averagely $20.3 \%$ lesser under hybrid mode. As a consequence, the COP improvement of hybrid mode is about $34 \%$ which meets the previous estimation; however, there still remains a distance from setting goal. Since ejector is capable of utilizing waste source energy and this kind of energy is free in a sense. If the generator heater of proposed system is powered by low grade heat energy, such as automobile exhaust, geothermal or solar energy, the power consumption of system will be further reduced. Thus $34 \%$ improvement is not the ultimate goal.

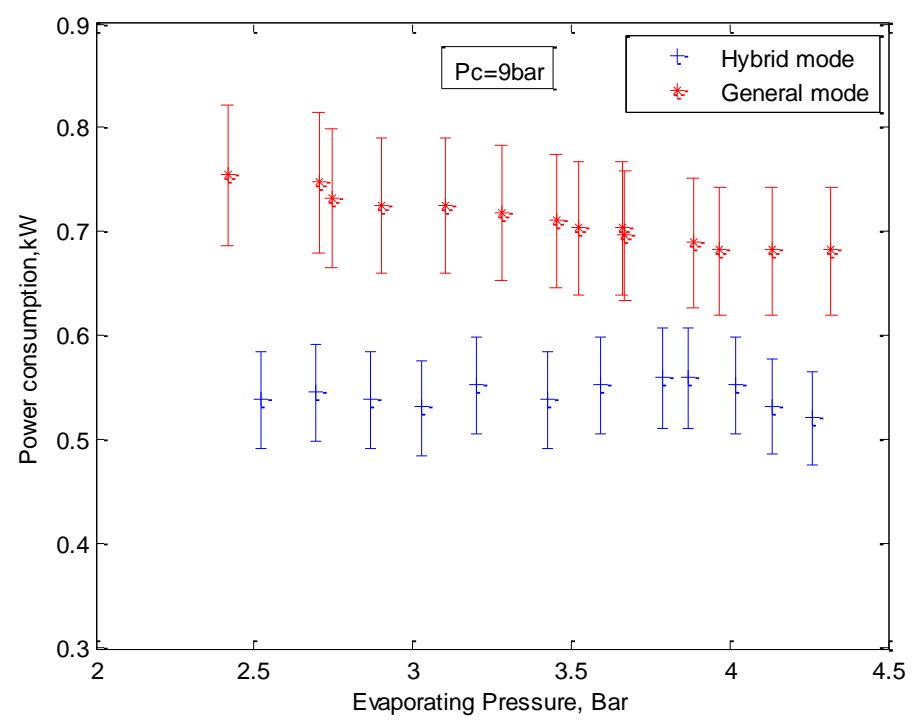

Figure 38 Power consumption between hybrid mode and general mode 


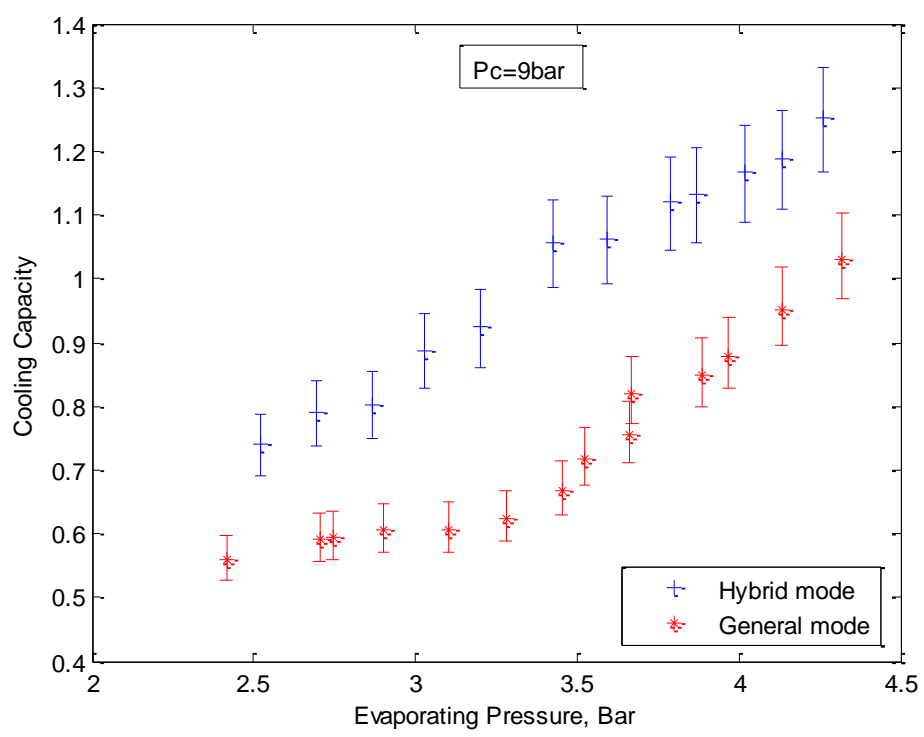

Figure 39 Cooling capacity between hybrid mode and general mode

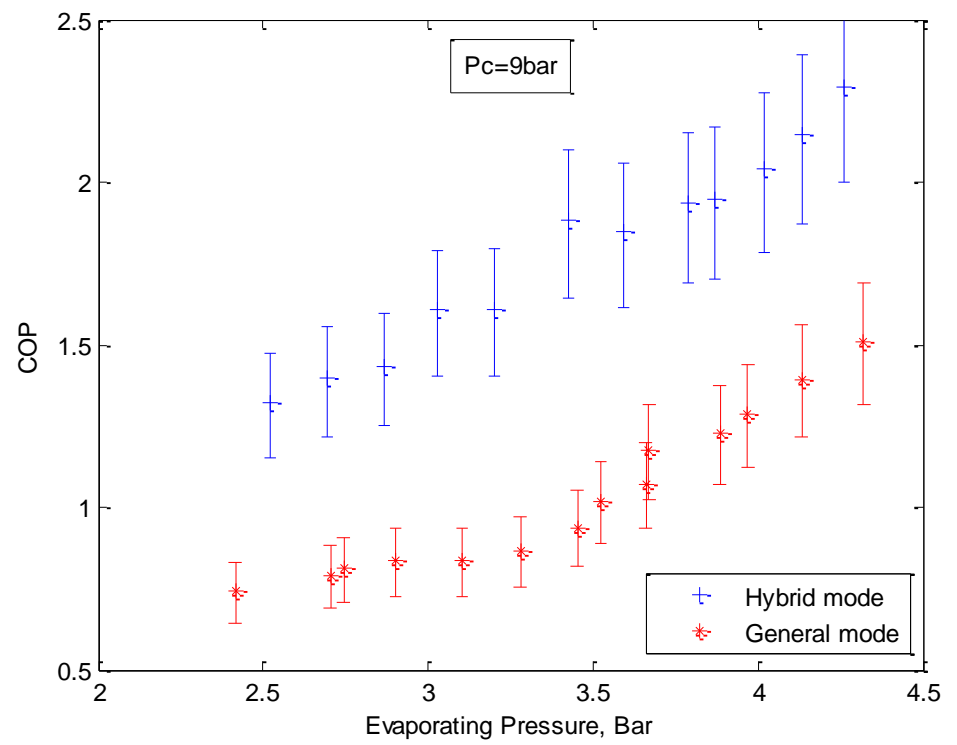

Figure 40 COP between hybrid mode and general mode

\subsection{Summary}

The different operation modes and detailed information of main components of proposed hybrid ejector-based air conditioning system are presented in this chapter. Also, in order to thoroughly investigate the character of proposed system, an experimental evaluation is conducted under different conditions. It has been seen that (i) the performance of ejector is sensitive to its area ratio and ejector A gives better performance than ejector B in terms 
of critical pressure and entrainment ratio; (ii) the performance of hybrid system is sensitive to evaporating and generating pressure. A higher evaporating pressure leads to larger entrainment ratio and COP while a higher generating pressure only results in higher COP; (iii) the compressor applied which enlarge the pressure difference between ejector secondary inlet and evaporator outlet indeed improve the overall performance and the COP improvement can reach up to $34 \%$ which indicated the potential of energy saving in hybrid ejector refrigeration system.

In order to further explore the feature of proposed system, modeling is an inevitable approach. It will be seen in the next chapter that a hybrid modeling method is applied on the system. And the discussion on the performance of this method is also presented. 


\section{Chapter 4 Hybrid Modeling of Hybrid System}

\subsection{Different modeling approaches}

Although the hybrid ejector-based air-conditioning system is consisted of many components, the major ones are condenser, evaporator, ejector, compressor and expansion valve, respectively. The performance such as coefficient of performance, energy consumption and cooling capacity etc. of hybrid system is significantly affected by the operation of each component. There are many studies on modeling of air conditioning system either at component level or system level. Notwithstanding the investigation of compressors and expansion valves has been conducted, the more attentions are paid on the heat exchanger and ejector models. Generally, there are three kinds of models, namely mathematical models, the black box models and the hybrid models, respectively.

\subsubsection{Mathematical models}

The heat transfer process is described by equations according to principle of fluid dynamics and thermodynamics. Since all the detailed process can be derived from mathematical expression, such as refrigerant pressure and temperature at certain locations, the accuracy of the model is guaranteed. And it is very helpful for thoroughly investigation based on mathematical models. [78] presented an advanced, nonlinear and moving boundary model of condenser based on the mass and energy conservation relationship. The model is applicable to both finned tube bundle and plate type condenser through including fins and non-circular flow passages. The universality of model can be achieved by simply varying the function applied. The performance shows that the model can accurately predict the steady state experiment results. Further, the model for evaporator will be developed. A dynamic one-dimensional mathematical model for desiccant coated heat exchanger system was proposed by [79] to predict the performance of system. The developed " $\mathrm{z}$ " water flow direction is applied in describing heat and mass transfer process. By introducing the initial, boundary and auxiliary conditions, the heat and mass transfer relations between water and desiccant layer are obtained. Then the governing equations are dispersed using a backward finite different method and solved by Newton-Gauss method. The results show that the simulated data agrees well with experimental results and the error is within $\pm 15 \%$. Furthermore, [80] presented a graph 
theory based steady state mathematic model for fin and tube heat exchanger design. With the help of the directed graph and graph-based traversal methods, the proposed model is able to cover the different arrangement of refrigerant circuit as well as quantify the refrigerant distribution states. To shorten the simulating cost, an alternative iteration method is adopted to solve the conservation equations. After verification, the model can achieve the goal of limiting the maximum error within $\pm 10 \%$. However, the drawbacks of these mathematical models are that the complexity of equations can be high, the iterative process of computing the unknown parameters and states variables is time consuming, and it is impractical to employ these models for online optimization issue.

\subsubsection{Black box models}

Black box models are constructed based on existing historic data. It is usually applied to predict the complex relationship between input and concerned output via polynomial functions or artificial neural networks. By doing so, the complicated operating principle can be neglected. Thus, when the detailed knowledge of heat exchanger is deficient or the experimental data is sufficient, the heat exchanger can be expressed by sets of polynomial functions or artificial neural network with certain structure whose parameters are obtained through data fitting. [81] applied artificial neural networks to predict the heat transfer rate of the wire-on-tube type heat exchanger. The most commonly used learning method -back propagation algorithm- was selected in the training and testing of the network. The results show that the mean absolute relative error was within 3\% between ANNs approach results and experimental data. [82] employed neural networks to simulate the thermal performance of a compact, fin and tube heat exchanger with air and water/ethylene glycol anti-freeze mixtures as the working fluids. The results indicated that the developed networks were able to predict the overall rate of heat transfer in the exchanger with a high degree of accuracy over a range of flow rates and inlet temperature and under various fluids concentrations. [83] chose artificial neural network technique to realize the simulation of the time dependent behavior of a heat exchanger. Additionally, it was also used to control the temperature of air passing over it. Although the network was trained off-line, the author are optimistic that on-line training will be incorporated in the future to enable continuous learning and adaptation. Although black box models can be accurate within training data range, the prediction accuracy is usually reduced once the target is 
beyond the training scope. Besides, there is lack of profound understanding about inner operating principle of modeled system via black box models.

\subsubsection{Hybrid models}

The mathematical models are accurate but complex; the black box models are simple but lack of generality. The hybrid models inherit the advantages of both mathematical and black box modeling methods since it is based on related physical theories while obtain certain coefficients which are impractical to measure via experimental data fitting. [84] developed a simplified hybrid model to simulate the single U-tube ground heat exchangers. The model is suitable for short time step simulation and the comparison result between model prediction and refined computational fluid dynamic reference model indicates the accuracy and robustness of proposed model. [85] proposed hybrid modeling approach for heat exchanger considering the fouling effect. The model was based on mass and energy conservation, thermodynamic theories and fouling principles. [86, 87] improved hybrid models for condensers and evaporators based on Newton law of cooling. The result implied that the proposed hybrid model satisfied the demand of on-line optimization problem. Since there are so many preponderance aspects of hybrid model, the general steps of constructing a hybrid model is illustrated as follows: 1) Construct the governing equations based on appropriate theoretical knowledge, such as mass and energy conservation theory, thermodynamic principle and so on; 2) Identify the most influential measureable and controllable parameters either via parameter selection algorithms or expertise; 3) Use the selected variables to represent the unmeasureable ones with the help constants of coefficients; 4) Rearrange and simplify the representation into a single equation; 5) Identify the unknown coefficients by relative methods, such as curve fitting, least square method and so on, based on experiment data. In this thesis, the system components models are developed through hybrid modeling and the fouling effect is also considered.

\subsection{Condenser}

The structure diagram of a condenser is shown in Figure 41. A condenser consists of two cycles: the working fluid cycle where refrigerant involves and the secondary fluid cycle where ambient air involves. 


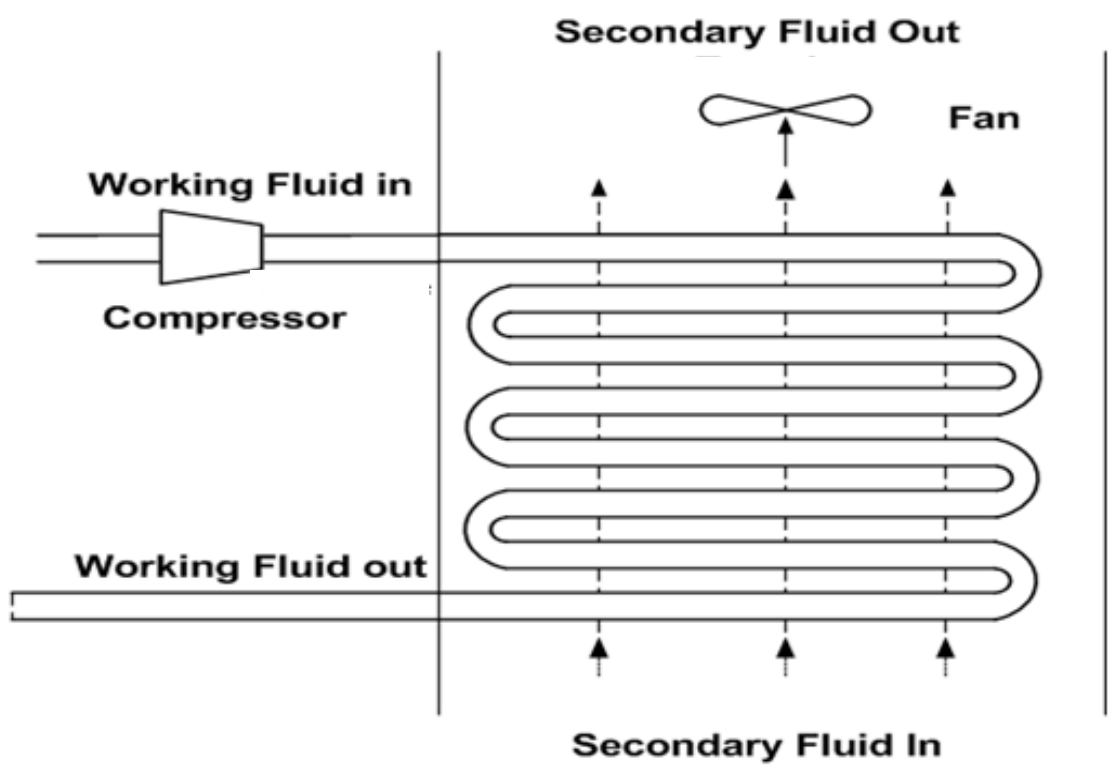

Figure 41 Structure diagram of condenser [87]

In the working fluid cycle, the refrigerant with high temperature and pressure enters the condenser with a certain mass flow rate. Since the temperature of refrigerant is higher than that of the secondary fluid, heat is transferred from the refrigerant to the secondary fluid through tube wall mostly in a manner of convection. With heat emitting, the refrigerant condenses at a high pressure. At the outlet of condenser, the refrigerant condenses to liquid phase even to sub-cooled liquid. In the secondary fluid cycle, with the use of fan, the secondary fluid is forced flowing through the condenser with a certain mass flow rate. At the outlet of condenser, the temperature of secondary fluid will increase to a certain value because of the heat exchange with working fluid.

In a refrigeration system, the condenser can operate in three different conditions, namely single region condition, two regions condition and three regions condition. Single region condition implies that the heat transfer during the condenser is very low so the refrigerant is all super-heated from the inlet to outlet of the condenser. Two regions condition indicates that the refrigerant enters the condenser in super-heated state and leaves it in liquid-vapor mixture state. For the three regions condition, the refrigerant at inlet of condenser is assumed to be super-heated vapor and at the outlet always condenses to subcooled liquid. Thus the condenser has three different fluid regions, super-heated, two phases and sub-cooled. As the three regions condition can satisfy normally most of 
experiment situations, it is the best candidate to be modeled. The detail structure of three regions is shown in Figure 42.

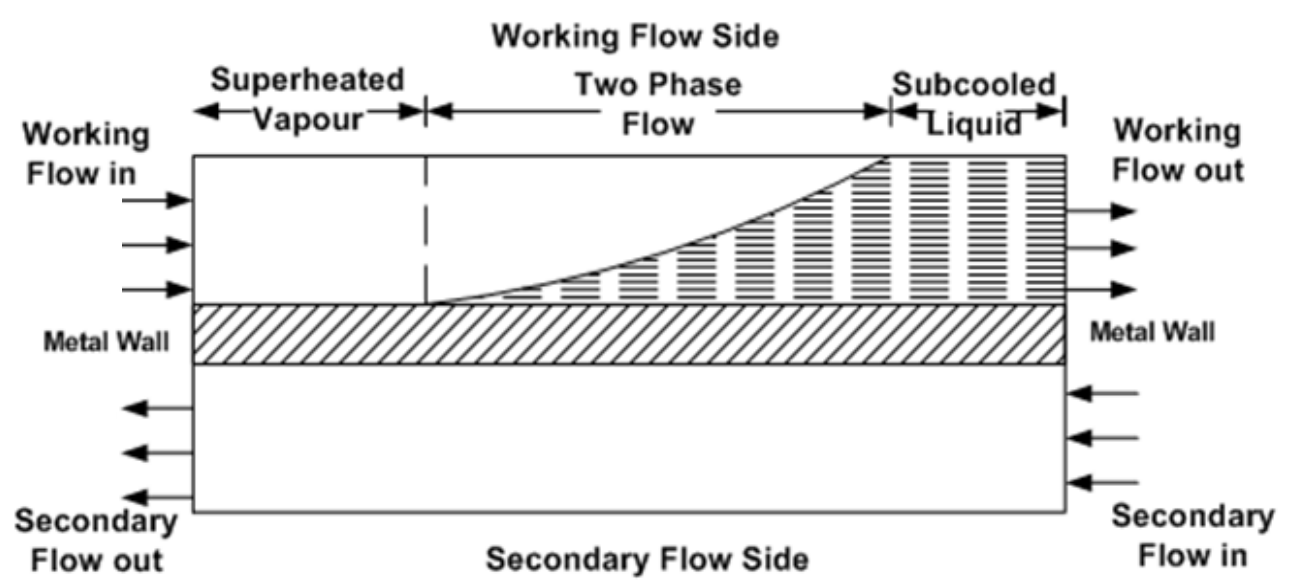

Figure 42 Three regions condenser [87]

Based on mass and energy conservation principle and Newton's cooling law, a simple yet accurate hybrid model can be derived for condenser heat transfer process as [87]

$$
Q_{c}=\frac{c_{1} \dot{m}_{r}\left(\mathrm{~T}_{s a t}-\mathrm{T}_{\text {SFin }}\right)+c_{2} \dot{m}_{r}\left(\mathrm{~T}_{r i n}-\mathrm{T}_{s a t}\right)+\dot{m}_{r} \Delta h_{v l}}{1+c_{3} \frac{\dot{m}_{r}}{\dot{m}_{S F}^{e}}}
$$

where $c_{1}, c_{2}$ and $c_{3}$ are unknown parameters which can be identified by experiment data, $T_{\text {sat }}, T_{S F i n}, T_{\text {rin }}, \dot{m}_{r}, \dot{m}_{S F}$ and $h_{v l}$ are the refrigerant saturated temperature, secondary flow inlet temperature, refrigerant inlet temperature, refrigerant mass flow rate, secondary flow rate and refrigerant enthalpy difference between vapor and saturated liquid phase, respectively, $Q_{c}$ is the heat transfer rate of the condenser, $e$ is the power of Reynold number in Dittus-Boelter equation.

Usually, $e$ is adopted as 0.8 in common practice. If it is regarded as unknown parameters, the nonlinear least square method, also known as Levenberg-Marquardt method, can be applied to solve the identification problem, and it is shown as:

$$
f(t)=\sum_{i=1}^{n} r_{i}^{2}(t)=\sum_{i=1}^{n}\left(\frac{c_{1} \dot{m}_{r}\left(\mathrm{~T}_{\text {sat }}-\mathrm{T}_{\text {SFin }}\right)+c_{2} \dot{m}_{r}\left(\mathrm{~T}_{r i n}-\mathrm{T}_{\text {sat }}\right)+\dot{m}_{r} \Delta h_{v l}}{1+c_{3} \frac{\dot{m}_{r}}{\dot{m}_{S F}^{e}}}-Q_{\text {measured }}\right)^{2}
$$


where $f(t)$ is the function that calculate the sum of squares of the residuals between predicted and measured data, $n$ is the sample number, $Q_{\text {measured }}$ is the measured heat transfer rate.

The fitting results and relative error for condenser hybrid model are shown in Figures 43 and 44:

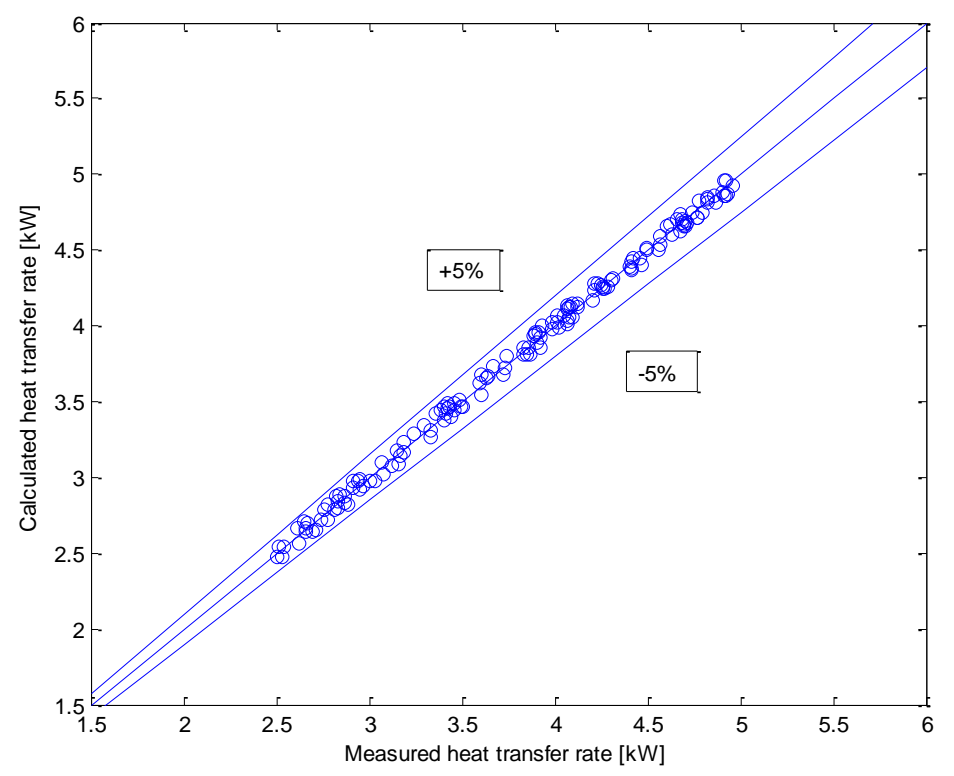

Figure 43 Experiment fitting result of condenser model

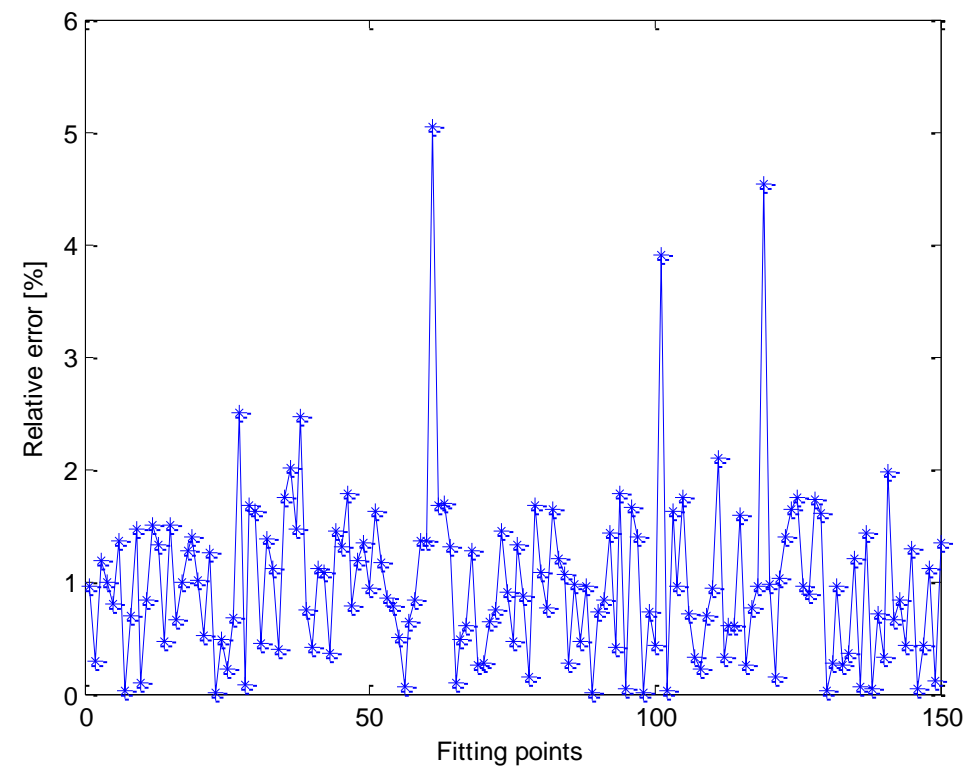

Figure 44 Relative error of condenser model 
From the results, it can be found that the heat transfer rate predicted by proposed model is within $\pm 5 \%$ relative error which indicates the promising accuracy of this model. The error is mainly attributed to the sensor errors during measurement and variations of fluid properties. Next, evaporator will be modeled. The working principle of evaporator is similar with condenser, so it is relative easier to model based on condenser model.

\subsection{Evaporator}

The working principle of evaporator is similar with condenser and the structure diagram of an evaporator is shown in Figure 45. It also consists of two cycles: the working fluid cycle and the secondary fluid cycle.

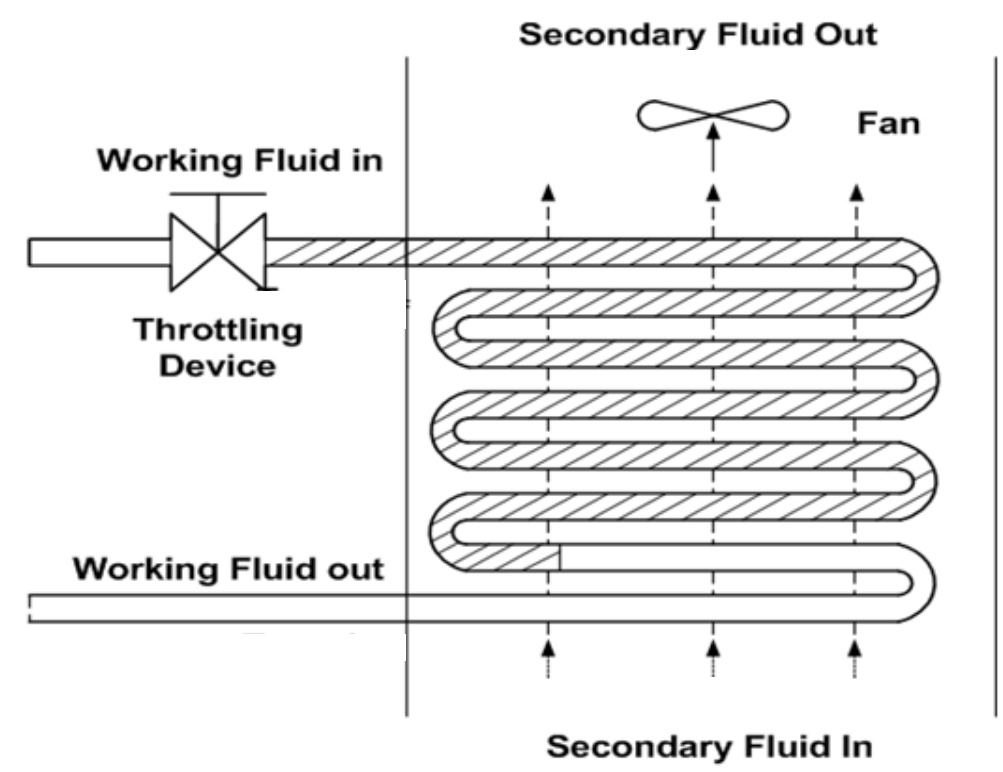

Figure 45 Structure diagram of evaporator [86]

In the working fluid cycle, the liquid refrigerant leaves the condenser and enters throttling device. Because of a sudden pressure drop within throttling device, the liquid fluid partially boils and flashes into vapor. Since the boiling temperature of refrigerant is lower than the temperature of secondary fluid, the heat is transferred from secondary fluid to the refrigerant in a manner of convection. With heat obtained from secondary fluid, the refrigerant at the outlet of evaporator is usually in super-heated condition. In the secondary fluid cycle, the evaporator fan forces the fluid to circulate and realizes the forced convection process. Usually, there is no phase change within secondary fluid cycle. 
In a refrigeration system, the evaporator can operate in three different conditions, namely single region, two regions and three regions. For single region situation, the refrigerant entering and leaving evaporator is liquid and vapor mixture. This condition indicates that the evaporator has low heat transfer efficiency and usually connected with a receiver at the outlet of evaporator. For two regions condition, the refrigerant at the inlet of evaporator is in two-phase and at the outlet of evaporator is in super-heated state. For three regions condition, the refrigerant at the inlet of evaporator is sub-cooled liquid. With heat transfer to refrigerant, the refrigerant gradually vaporizes and completes vaporization within evaporator. As refrigerant continues to absorb heat, it becomes super-heated at the outlet of evaporator. Since the two regions condition is the most common operation mode based on experiment data, it is the best candidate to be modeled. The detail structure of two regions condition is shown in Figure 46.

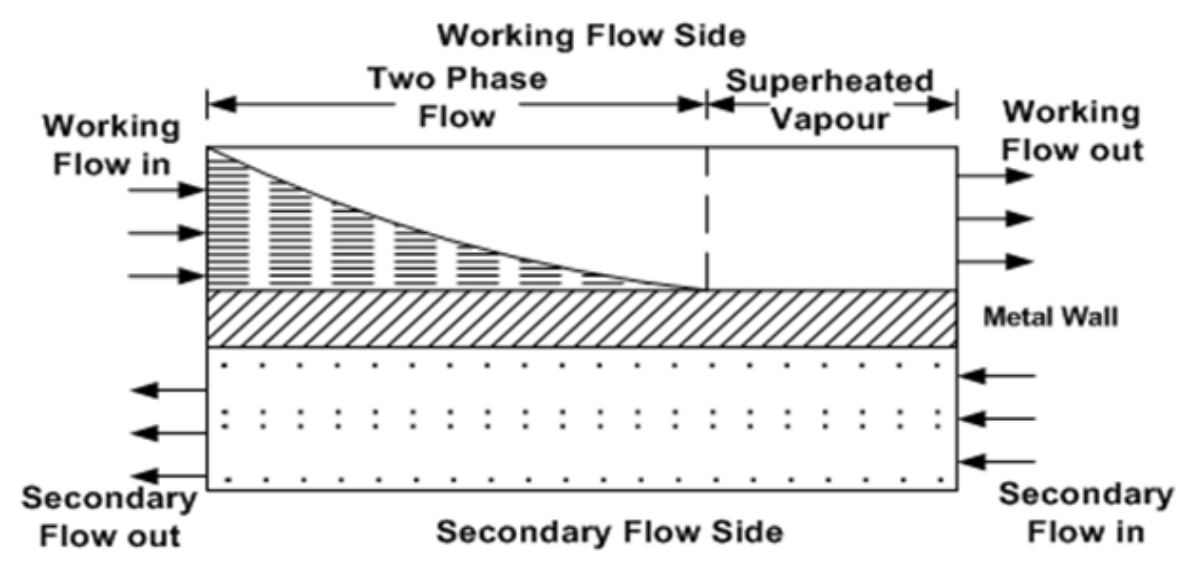

Figure 46 Two regions evaporator [86]

Since the working principle of evaporator is similar to that of condenser, the heat transfer rate of evaporator can be obtained as [86]

$$
Q_{e}=\frac{\dot{m}_{r}\left(h_{v}-h_{r i n}\right)+c_{1} \dot{m}_{r}\left(T_{S F i n}-T_{s a t}\right)}{1+c_{2} \frac{\dot{m}_{r}}{\dot{m}_{S F}^{e}}}
$$

where $c_{1}$ and $c_{2}$ are unknown parameters to be identified, $T_{S F i n}, T_{s a t}, h_{v}, h_{r i n}, \dot{m}_{r}, \dot{m}_{S F}$ are the temperature of inlet secondary flow, temperature of saturated refrigerant, enthalpy of vapor refrigerant, enthalpy of inlet refrigerant, refrigerant mass flow rate and secondary flow rate, respectively. $Q_{e}$ is the heat transfer rate of evaporator. The identification 
process is similar to that of condenser, Levenberg-Marquart method is applied and the fitting results and relative error are shown in Figures 47 and 48.

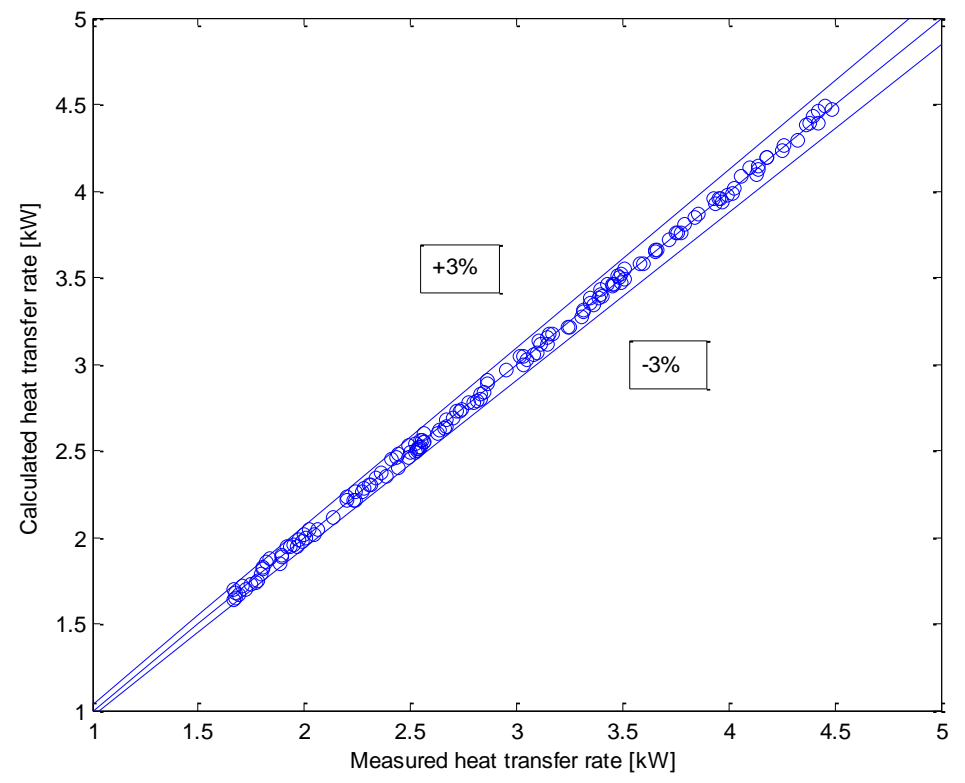

Figure 47 Experiment fitting result of evaporator model

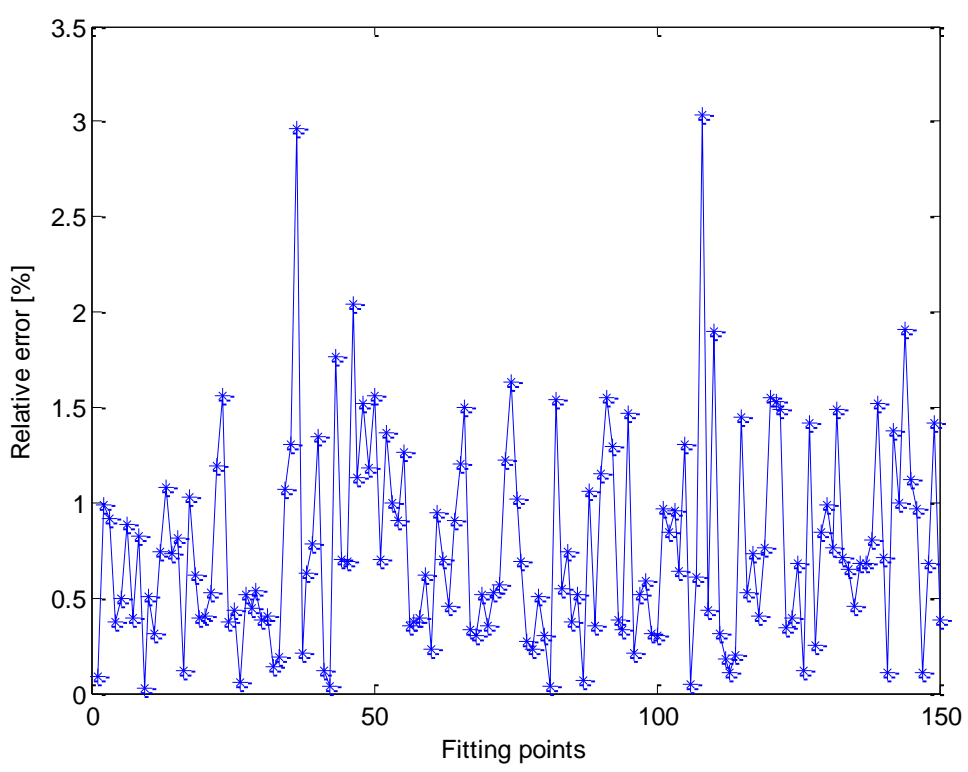

Figure 48 Relative error of evaporator model

From the result, it can be observed that the relative error of evaporator model is within $\pm 3 \%$, additional, most of the predicting errors are narrow down to $\pm 1.5 \%$ which indicating the good performance of proposed hybrid model. 


\subsection{Compressor}

For compressor, two aspects are of concerned that are mass flow rate of refrigerant passing through and the variation of refrigerant energy between inlet and outlet of compression process. A hybrid model for mass flow rate can be expressed as [88]

$$
\dot{m}=\left[c_{1}-c_{2}\left(\frac{P_{\text {outlet }}}{P_{\text {inlet }}}\right)^{c_{3}}\right] \times \omega
$$

where $P_{\text {outlet }}$ is the outlet pressure which equals to the ejector secondary flow inlet pressure, $P_{\text {inlet }}$ is the inlet pressure which equals to the evaporator outlet pressure and $\omega$ is the compressor motor speed. $c_{1}, c_{2}$ and $c_{3}$ are three unknown parameters to be determined. A hybrid model for refrigerant energy change is indicated as:

$$
\dot{Q}_{r}=c_{3} \omega \dot{m}_{r} P_{\text {inlet }}\left[P_{\text {com,ratio }} c^{c_{4}}-1\right]
$$

where $c_{3}=\frac{n}{n-1} v, c_{4}=\frac{n-1}{n}, P_{\text {com,ratio }}=\frac{P_{\text {outlet }}}{P_{\text {inlet }}}, n$ is a constant polytropic exponent and $v$ is the specific volume of compressor.

Again, the Levenberg-Marquardt method is applied to determine the unknown parameters in these two models. The fitting results of two models are shown in Figures 49 52, respectively.

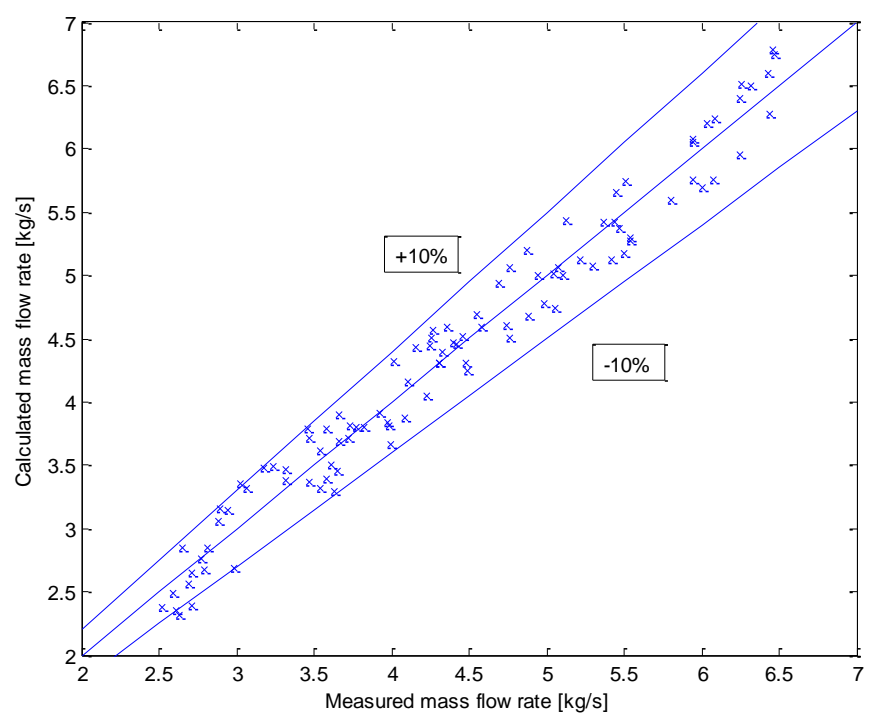

Figure 49 Experiment fitting result of compressor mass flow rate model 


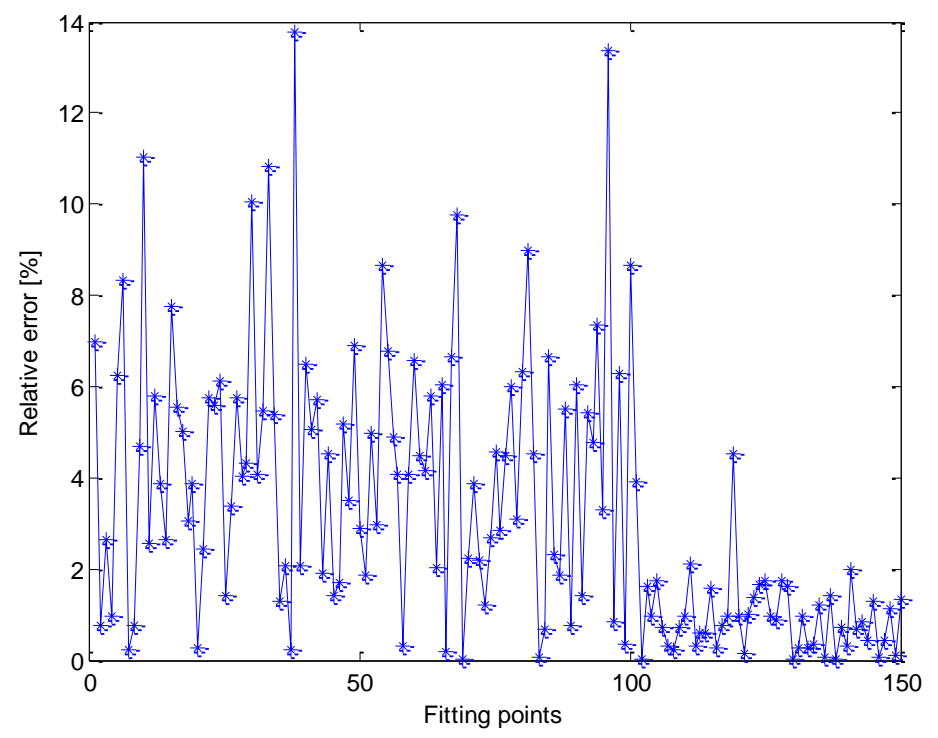

Figure 50 Relative error of compressor mass flow rate model

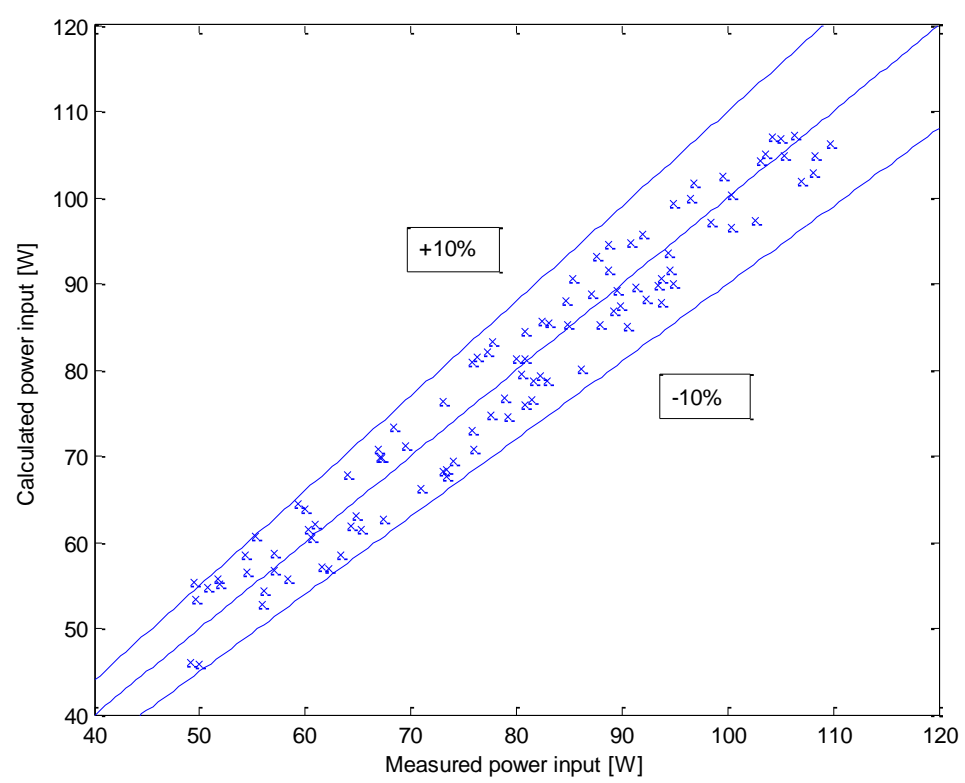

Figure 51 Experiment fitting result of compressor power consumption model 


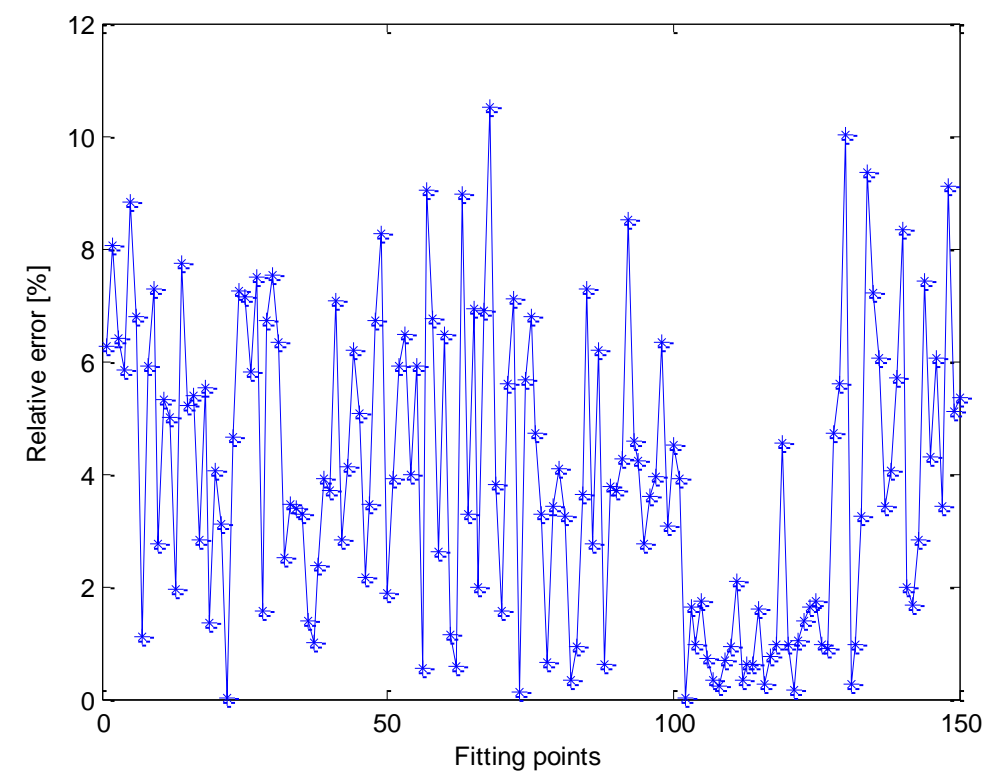

Figure 52 Relative error of compressor power consumption model

Due to the unstable operation condition of compressor and measuring error, the relative errors of two proposed models are around 10\%. Through applying more accurate measuring equipment and maintaining steady experimental conditions, the relative error can be reduced.

\subsection{Electric expansion valve (eev)}

The refrigerant mass flow rate is the only concern towards electric expansion valve and its value is depended on the allowance extent of aperture, the variation of refrigerant pressure between two sides of eev and the density of refrigerant at eev inlet point. Thus, the refrigerant mass flow rate through the eev is obtained from the model shown below:

$$
\dot{m}_{r}=\left(c_{1}+c_{2} \eta\right)\left(\rho_{r} \Delta P\right)^{1 / 2}
$$

where $c_{1}$ and $c_{2}$ are constant coefficients to be determined through experiment data, $\eta$ is the opening percentage of expansion valve, $\rho_{r}$ is the density of inlet refrigerant and $\Delta P$ is the pressure difference which equals to pressure at condenser outlet minus pressure at evaporator inlet. The expansion process is assumed to be isenthalpic, so the energy change $Q_{e e v}$ is zero. 


\subsection{Ejector}

There are various fashions of models for ejector modeling and can be normally classified into three different manners, namely, mathematical models which are solely based on theoretical knowledge, regression models which are solely relied on empirical experience and a combination of these two modeling manners called hybrid models. Based on each ones' features, hybrid model appears to be the most suitable candidate for our system. Since the process happening in ejector is rapid (the supersonic fluid contributes a lot), it is more practical to develop a static model for optimization purpose [89].

As the performance of ejector is highly affected by the back pressure, the critical operation mode is guaranteed during the experiment. The schematic diagram of a critical mode operation ejector is shown in Figure 53.

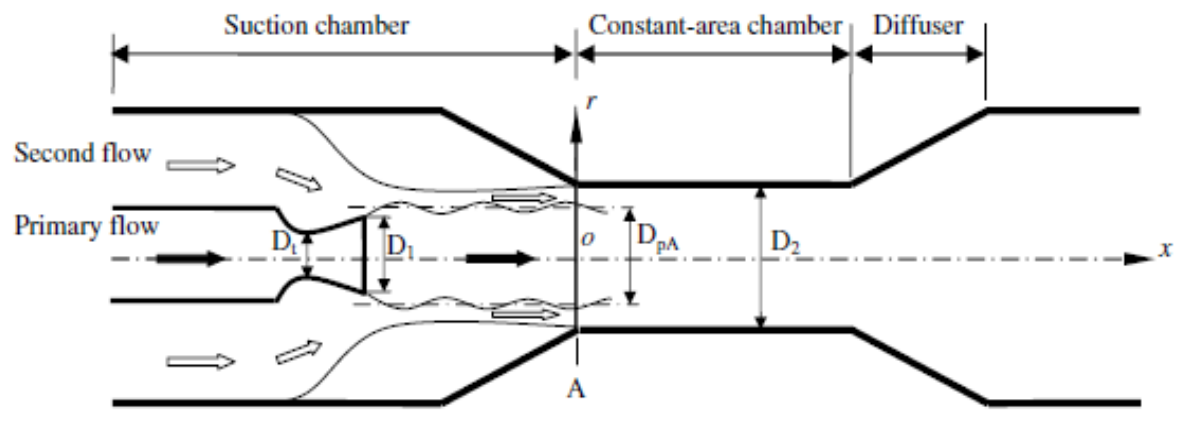

Figure 53 Diagram of critical mode operation [89]

From literature review, the most commonly governing equations for ejector modeling are listed below:

Under choking condition, the mass flow rate of primary flow can be given by [90]:

$$
\dot{m}_{p}=P_{p} A_{t}\left(\frac{\gamma \psi_{p}}{R_{g} T_{p}}\right)^{\frac{1}{2}}\left(\frac{1+\gamma}{2}\right)^{\frac{1+\gamma}{2(1-\gamma)}}
$$

where $P_{p}$ and $T_{p}$ are the stagnation pressure and temperature of the primary flow at the nozzle inlet, $A_{t}$ is the area of nozzle throat, $R_{g}$ is the universal gas constant, $\gamma$ is the specific heat ratio of vapor refrigerant and $\psi_{p}$ is the isentropic coefficient of compressible flow, respectively. 
According to shock circle model, the mass flow rate of secondary flow is derived as follow:

$$
\dot{m}_{s}=\frac{2 \pi P_{s} V_{p A}}{R_{g} T_{s A}}\left[\frac{n D_{2}^{2}}{4(n+1)}\left(1-\frac{D_{p A}}{D_{2}}\right)^{1+\frac{1}{n}}-\frac{n D_{2}^{2}}{4(2 n+1)}\left(1-\frac{D_{p A}}{D_{2}}\right)^{2+\frac{1}{n}}\right]
$$

Hence, the entrainment ratio can be obtained through dividing equation (45) by equation (36). After rearrangement and simplification, a practical expression is obtained as:

$$
\omega=\frac{\dot{m}_{s}}{\dot{m}_{p}}=c_{1}\left(\frac{P_{s}}{P_{p}}\right)^{c_{2}}\left\{1-c_{3}\left(\frac{P_{s}}{P_{p}}\right)^{\frac{-1-\gamma}{4 \gamma}}\left[\left(\frac{P_{s}}{P_{p}}\right)^{\frac{1-\gamma}{\gamma}}-1\right]^{\frac{-1}{4}}\right\}
$$

where $c_{1}, c_{2}$ and $c_{3}$ are three unknown parameters. If ejector geometric information and refrigerant properties are available, then parameter $c_{3}$ can be identified. Thus only two parameters need to be determined. The linear least squares method is applied. While if $c_{3}$ cannot be identified, the Levenberg-Marquardt method is applied. For our experiment, the refrigerant properties of R-134a can be found by software NIST and the ejector geometric detail is provided by its producer. So under this scenario, the former method is chosen. The fitting results are shown in Figures 54 and 55, respectively.

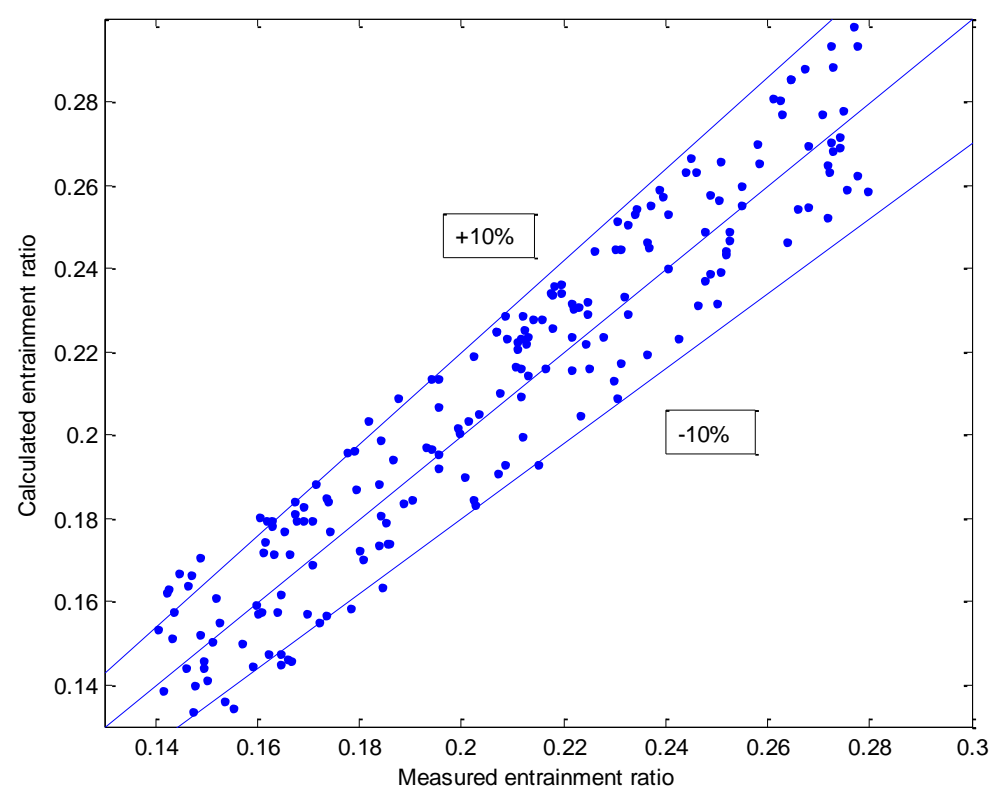

Figure 54 Experiment fitting result of ejector model 


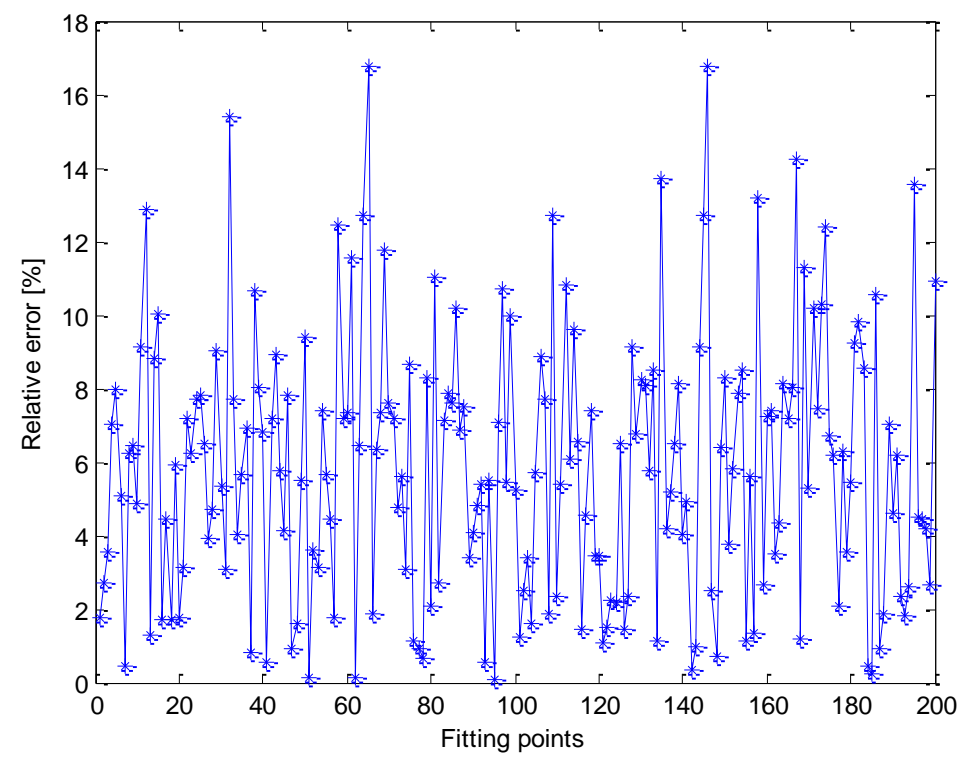

Figure 55 Relative error of ejector model

Due to the measuring error of mass flow rate of both primary and secondary flows and the assumptions made in order to simplify the model, the relative error reaches to around $10 \%$ within acceptable tolerance. The proposed ejector model is simple yet with satisfying accuracy when compared to others.

\subsection{Fouling effect on hybrid models}

\subsubsection{Different fouling models}

The proposed hybrid models of heat exchanger will only have a good performance under the assumption that the thermal conductance is always at its ideal conditions which is impossible in real applications [91]. Therefore, to extend the feasibility of hybrid model, the fouling effect on model performance must be considered. In order to modify the hybrid model, it is necessary to model the fouling process.

Due to the sedimentation, organic or biological growths, crystallization, corrosion or a combination of above mentioned effects, some deposits are generated and left on the heat transfer equipment, and these deposits are called fouling. Fouling will deteriorate the thermal performance of heat exchanger and may cause serious consequence if no action is implemented in time. For instance, in steam generators, fouling can change the thermal- 
hydraulic characteristics of component and lead to local spots with higher temperature, even cause the mechanical failure.

The most widely approved fouling model was proposed by [92] which was based on material balance:

$$
\frac{d R(\mathrm{t})}{d t}=\phi_{d}-\phi_{r}
$$

where $R$ is the fouling resistance, $t$ is time, $\phi_{d}$ is the fouling deposition rate which depends on the fouling mechanism and $\phi_{r}$ is the fouling removal rate which depends on features of deposit and shear stress. A number of fouling models have been proposed to describe the time-dependent fouling process, and will be listed as follow.

\section{Linear fouling model}

The described equation is focused on the variation of heat conductance abilities of heat exchanger due to crystalized process of pure salt contained sediment [93].

$$
R=R_{i}\left[1-\sqrt{\alpha} \Phi^{-1}(p)\right](t / M)
$$

where $R_{i}$ is the resistance of heat exchanger under ideal condition, $\alpha$ is the scatter parameter, $\Phi$ is the possible function describing the risk of fouling, $p$ is risk level and $M$ is medium value of time.

\section{Power-law fouling model}

This model is interested in the fouling growth caused by $\mathrm{CaCO}_{3}$ under high operating temperature. Researchers found that replicate corrosion can also be described by this model [94].

$$
R=R_{i}\left[1-\sqrt{\alpha} \Phi^{-1}(p)\right] t^{n} / M^{n}
$$

The power-law fouling model is treated as linear model in a transformed coordinate system.

\section{Falling-rate fouling model}

If the precipitation rate is larger than the removal rate, falling-rate fouling model is applied. It should be noticed that this falling-rate trend is normally observed from particular fouling. The expression is similar to power law model in a linearly transformed time scale [95]. 


$$
R=R_{i}\left[1-\sqrt{\alpha} \Phi^{-1}(p)\right] \operatorname{In}(t) / \operatorname{In}(M)
$$

\section{Asymptotic fouling model}

This model describes the crystallization of salts in cooling water heat exchangers. Such deposits will become balance due to scale removal mechanism [96].

$$
R=R^{*}\left[1-\exp \left\{-\ln \left[1 /\left(1-R_{i} / R^{*}\right)\right]\left[1-\sqrt{\alpha} \Phi^{-1}(p)\right] t / M\right\}\right]
$$

\subsubsection{Combination of fouling model and proposed hybrid model}

Generally speaking, the fundamental principle of heat exchanger is the energy and mass conservation from both working and secondary fluid side. According to Fourier's law, the heat transfer can be expressed as

$$
Q=h A \frac{d T}{d x}
$$

where $Q$ is the heat transfer amount, $h$ is the overall thermal coefficient, $A$ is the cross sectional area and $d T / d x$ is the temperature gradient in the flow direction.

The heat transferred between working fluid and secondary fluid is consisted of three parts: the convection from working fluid to tube internal surface, the conduction from tube internal surface to tube external surface and the convection from tube external surface to secondary fluid. Among these three parts, the thermal conductance of tube is much higher than that of working and secondary fluid. Thus, the performance of heat exchanger is largely depended on working and secondary fluid convection parts.

The convective heat transfer coefficient is often calculated from the Nusselt number which is related with fluid viscosity, character diameter, thermal conductivity, Reynolds number and Prandtl number etc. For the forced internal turbulent flow convection process, an empirical equation can be expressed explicitly as:

$$
N u_{D}=\frac{h d}{k}=C\left(\frac{\dot{m} d}{\mu}\right)^{e}\left(\frac{\mu c_{p}}{k}\right)^{n}
$$

where $d$ is the character diameter, $k$ is the fluid thermal conductivity, $\mu$ is the fluid viscosity, $\dot{m}$ is the mass flux and $c_{p}$ is the specific heat coefficient at constant pressure. $C$, 
$e$ and $n$ are constant coefficients that are often independent of the fluid properties. In (72) and (73), the Reynolds number $\operatorname{Re}_{D}$ and the Prandtl number $\operatorname{Pr}$ are calculated by:

$$
\operatorname{Re}_{D}=\frac{\dot{m} d}{\mu}
$$

And

$$
\operatorname{Pr}=\frac{\mu c_{p}}{k}
$$

After rearrangement of (71), the heat transfer coefficient can be simplified as:

$$
h=b_{w} \dot{m}^{e}
$$

where

$$
b_{w}=\frac{4^{e} C c_{p}^{n} k^{1-n} \mu^{n-e}}{\pi^{e} d^{1+e}}
$$

Since the secondary flow also follows the convective heat transfer process, the heat transfer is in similar form with working flow.

$$
h=b_{s} \dot{m}^{e}
$$

where

$$
b_{s}=\frac{C c_{p}^{n} k^{1-n} \mu^{n-e}}{S^{e} d^{1-e}}
$$

After combining equations (70), (74) and (76), it can obtained that

$$
\dot{Q}_{i}=\frac{1}{\frac{1}{h_{w} A_{w}}+\frac{1}{h_{s} A_{s}}} \frac{d T}{d x}
$$

Further, substitute (74) and (76), it can be rearranged that

$$
\dot{Q}_{i}=\frac{b_{s} \dot{m}_{s}^{e} A_{s}}{1+\frac{b_{s} \dot{m}_{s}^{e} A_{s}}{b_{w} \dot{m}_{w}^{e} A_{w}}} \frac{d T}{d x}
$$


If considering the effect of fouling and the generality of fouling model, polynomial functions are used to approximate the real fouling effect. The mathematical expression of proposed model is

$$
\dot{Q}_{r}=\frac{\dot{Q}_{i}}{c_{1}+c_{2} t+c_{3} t^{2}+\ldots+c_{n+1} t^{n}}
$$

where $\dot{Q}_{i}$ is the ideal heat transfer rate and $\dot{Q}_{r}$ is the actual heat transfer rate.

Although $n$ can be infinite, the polynomial functions with $n$ no bigger than 4 are considered to meet the precision requirement as well as training speed. The performance comparison between fouling models with different orders polynomial functions are help to determine the optimal choice.

\subsubsection{Model validation and results discussion}

Similar to the validation of original hybrid model, taking evaporator as an example, the hybrid ejector model integrated with fouling effect is applied and the LevenbergMarquadt method is applied to identify the unknown coefficients.

$$
f(c)=\sum_{i=1}^{n}\left(\dot{Q}_{r}-\dot{Q_{m}}\right)^{2}
$$

To obtain sufficient data for parameter identification, the experiment was conducted on proposed hybrid system for more than 200 hours. The heat transfer amount was controlled by varying the refrigerant mass flow rate and the secondary air flow rate. The clean refrigerant was applied to help identified the parameter in original hybrid model. Then the polluted refrigerant was adopted to investigate the effect of fouling on system model.

All the enthalpies of refrigerant and secondary fluid are calculated from curve fitting based on measured corresponding temperature, pressure and humidity. And the mass flow rate is obtained through flowmeters installed on system.

The comparison between hybrid model without considering fouling effect and fouling integrated hybrid model as well as variation of heat transfer rate with time are shown in Figures 56 61, respectively. 


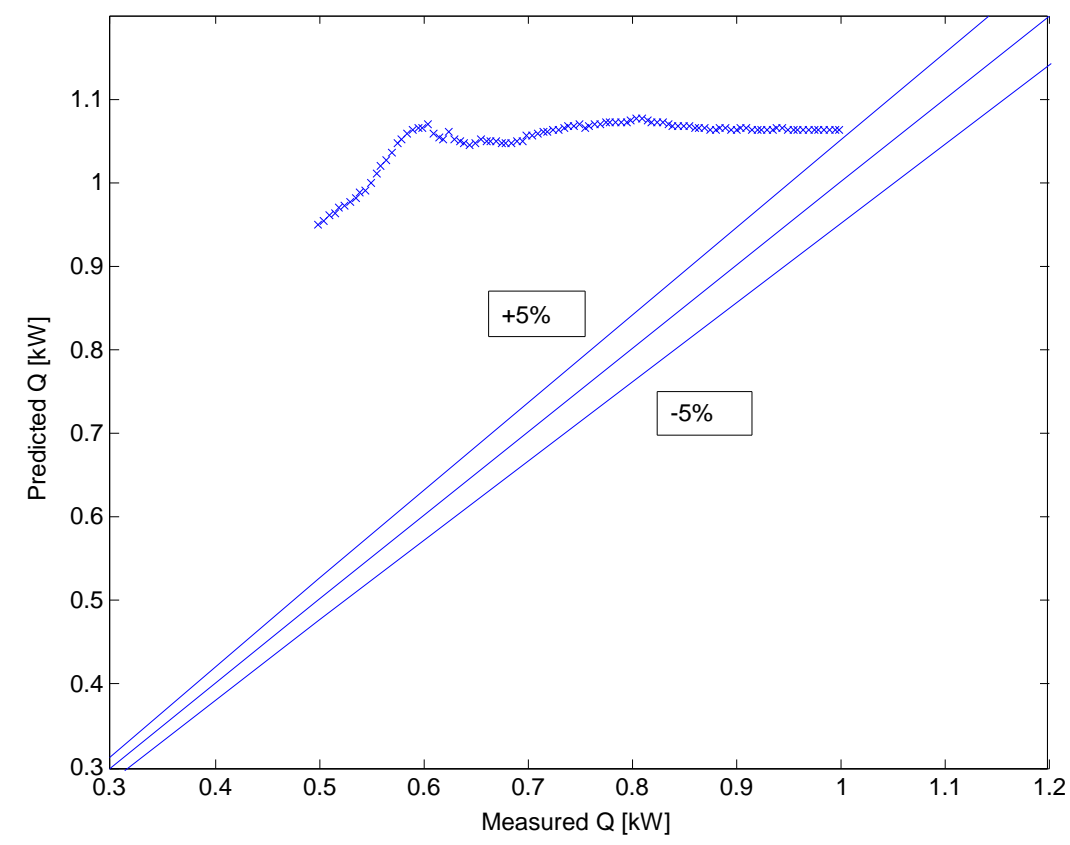

Figure 56 Fitting results of hybrid model without considering fouling condition

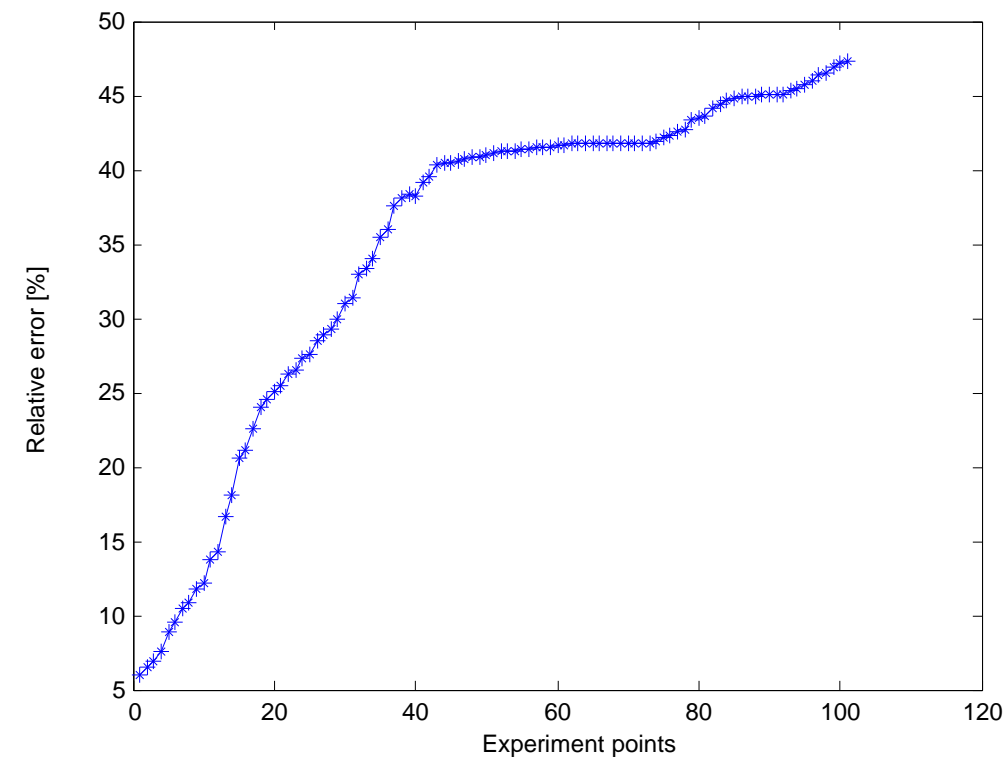

Figure 57 The result of relative error of hybrid model without considering fouling condition 


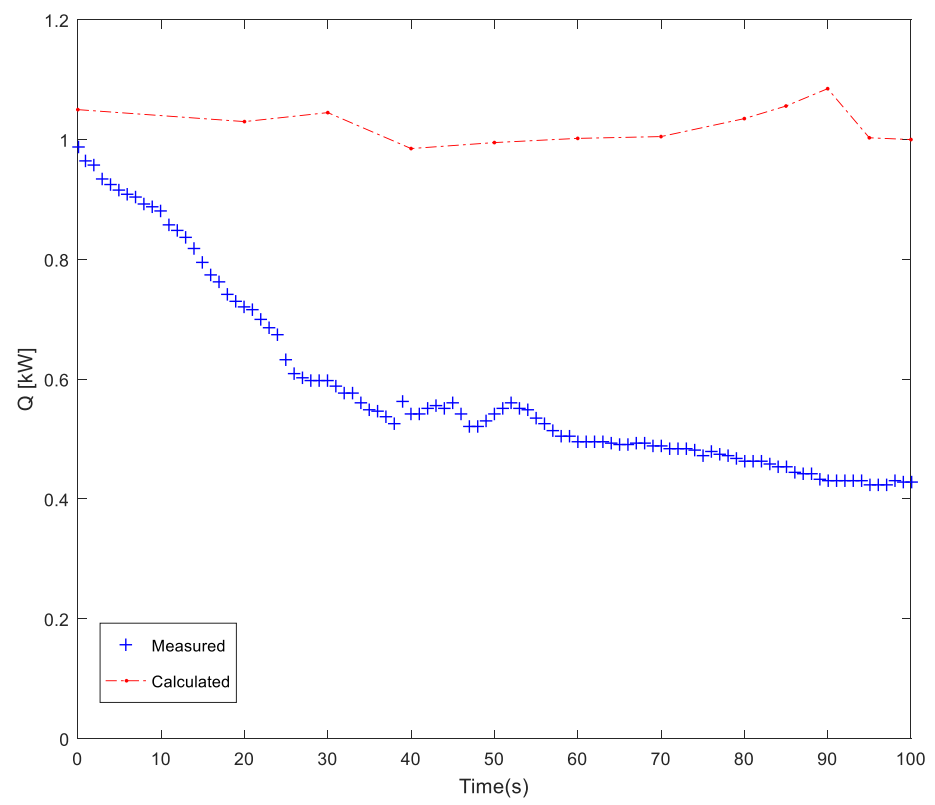

Figure 58 The result of measured and calculated heat transfer rate by hybrid model without considering fouling condition

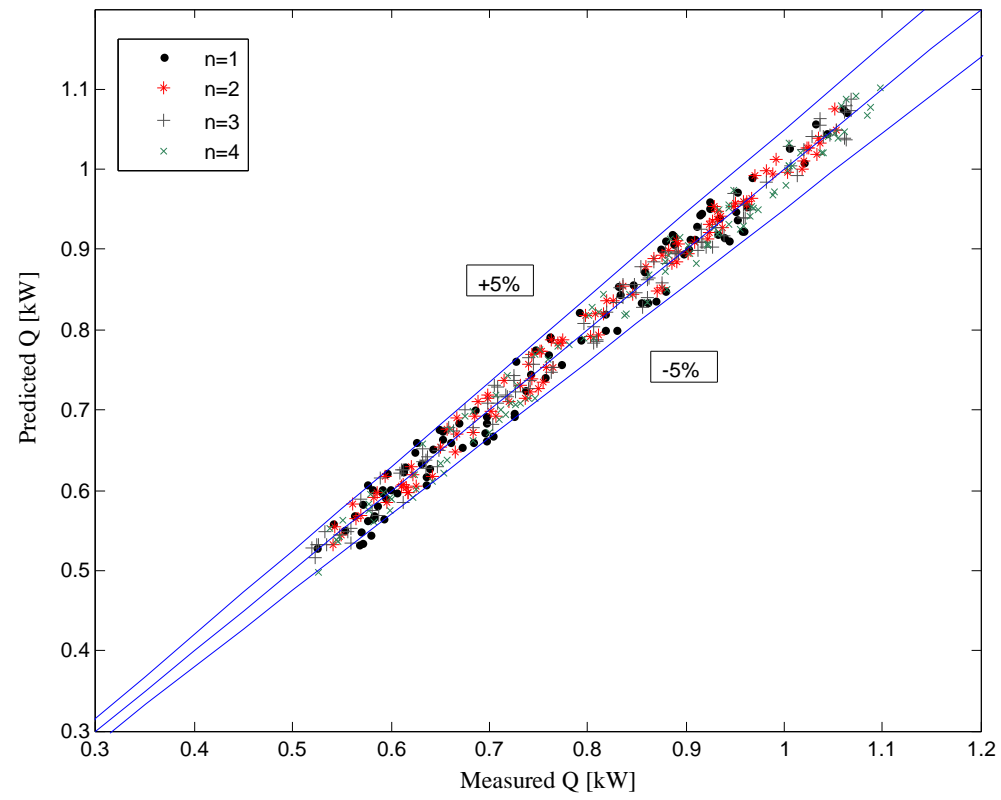

Figure 59 Fitting results of hybrid models with different orders considering fouling condition 


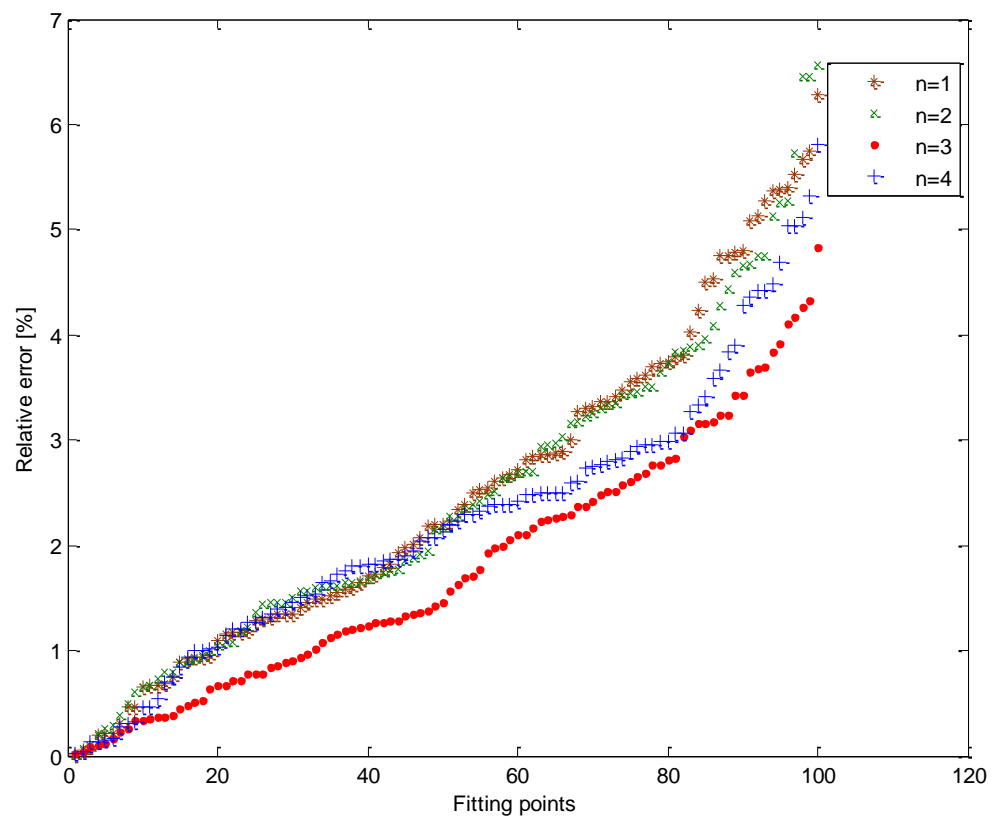

Figure 60 Relative error of hybrid model considering fouling condition

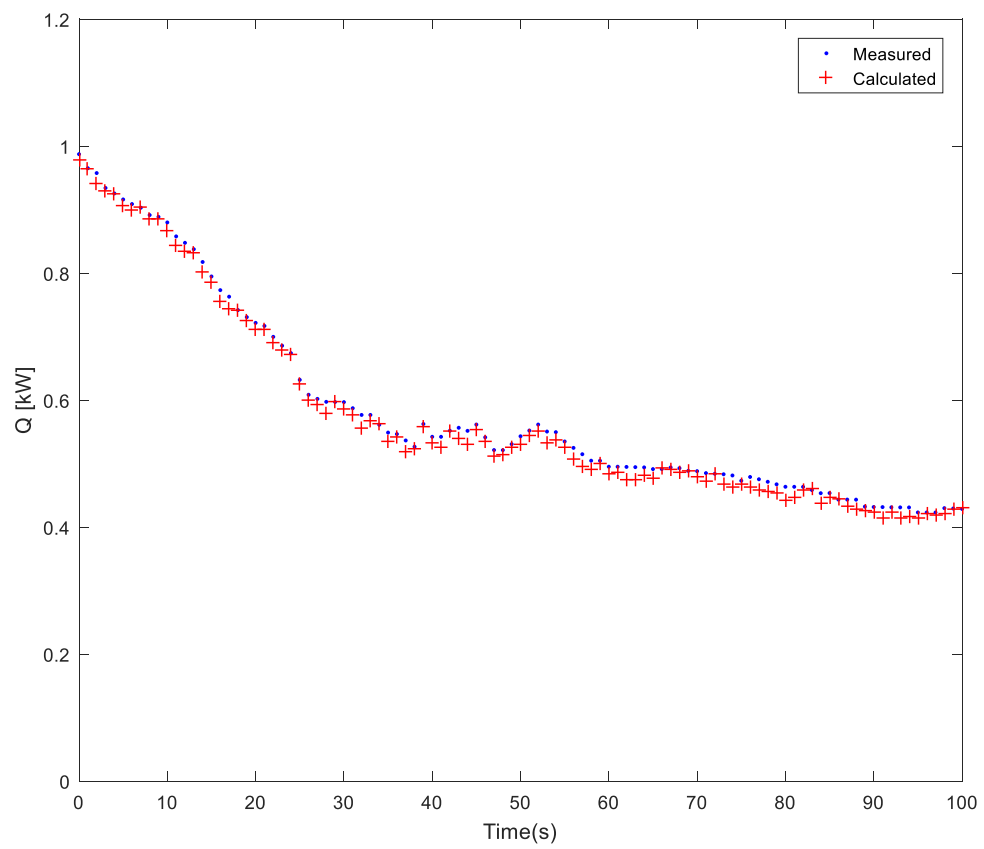

Figure 61 The result of measured and calculated heat transfer rate by hybrid model considering fouling condition

It can be seen that the fitting result of hybrid model without considering fouling effect tends to predict a higher heat transfer rate since it ignores the fact that the heat rate is 
reduced by the deposits. The deviation is larger when the heat transfer rate is small while gradually decreases with the increase of heat transfer rate. The relative error is ascend sorted first and then plotted. It can be found that the relative error of hybrid model without considering fouling effect can reach to nearly $50 \%$ compared with the $\pm 5 \%$ relative error of improved hybrid model. Moreover, the improved hybrid model with different orders of polynomial functions $(n=1,2,3,4)$ are applied to find out the most optimal one. And the result from Figure 60 indicates that the relative error of hybrid model with $n=3$ has the smallest relative error compared with others. Therefore, it is recommended to adopt $3^{\text {rd }}$ polynomial function to approximate the heat transfer rate under fouling effect.

\subsection{Summary}

The hybrid models of the main components of proposed hybrid ejector-based air conditioning systems are described in this chapter. From the result, it can be seen that 1) the complexity of hybrid models of evaporator and condenser can be largely reduced through depicting the complicated process with coefficients derived from curve fitting; 2) all the performance of proposed hybrid models indicate their abilities of accurately approximating the real value over a wide scope of operation; 3) the hybrid model integrated with fouling effect can predict the actual heat transfer rate with satisfying performance.

From the modeling process, it can be revealed that the accuracy and efficiency of hybrid models can satisfy the requirement of online optimization problem. This virtue is due to the fact that the unknown factors of hybrid models are obtained via curve regression according to acquired lab data or catalog data direct received from manufacturers rather than through rigid physical deduction. In the following chapters, the application of proposed hybrid models will be exhibited and possess an important position for optimization strategy development. 


\section{Chapter 5 Artificial Neural Network Modeling of Hybrid}

\section{System}

\subsection{Background of artificial neural network modeling}

In order to achieve controlling or optimizing purpose, the model of target system is indispensable. As mentioned before, more and more attentions have been drawn on modeling of air conditioning systems. There are many existing techniques available for modeling purpose, while the artificial neural network modeling approaches are popular over the others for their excellent performance in approximating nonlinear complex relations. Besides, the neural modeling methods are based on practice data obtained from either experimental platform or manufactures which avoiding the mathematical description of complicated process happening during operation of air conditioning systems. There have been many researches about neural modeling application for air conditioning systems. [97] developed an ANN model which was based on experimental data to predict the performances of electric vehicle air conditioning system. By varying the scroll compressor speed, EEV opening percentage and environment temperature, the performances such as refrigerant mass flow rate, compressor power consumption and refrigeration capacity were recorded. The developed model was tested with two transfer functions (logsig and tansig) and different hidden neurons. The optimized structure was determined with 13 hidden neurons and logsig transfer function which demonstrated the best prediction with $0.92-2.71 \%$ mean relative errors. A neural network with seven neurons in input layer, seven neurons in hidden layer and six outputs was developed by [98] to evaluate the performance of a desiccant air conditioning system. The input parameters are dry bulb temperature, humidity ratio, flow rate of the process, regeneration air streams of dehumidifier and regeneration temperature. Moisture removal rata and effectiveness of the dehumidifier are considered as the performance indicators. After comparison between results of ANN model and experimental test results, a close agreement is observed which indicates an excellent fitting ability of ANN. [99] managed to develop a novel ANN aided fuzzy logic controller for simultaneous control of indoor air temperature and humidity using a variable speed direct expansion air conditioning system. A novel control principle was proposed to decouple the temperature and humidity 
control loops by introducing two interim variables of sensible and latent output cooling capacity of the system. Both the command following tests and disturbance rejection tests were carried out and the results illustrated that the air dry-bulb and wet-bulb temperature were properly controlled with satisfying performance in terms of control accuracy and sensitivity. [100] applied neural network to model the HVAC systems as well as to control. They modeled a hot water system using ANN and selected outlet air temperature in air handling unit as control parameter. The results demonstrated that ANN is capable of capturing dynamic manner of the system. A feed forward neural network was introduced by [101] to predict the performance of a variable cooling capacity cooling system using water flow rate of condenser as the input. [102] proposed a neural network for predicting the performance of a chiller and an ice thermal storage vessel. Their work had implied that ANN was able to predict the vessel capacity under different working modes.

However, ANN has several limitations such as low speed of convergence and hard to simplify complex learning process. A novel yet efficient learning algorithm, named extreme learning machine (ELM), was proposed by [103] recently and shows excellent performance over the other algorithm under same constrained problem. The most distinctive characteristics of the ELM is the randomly assignment of both the inputweights and hidden biases which results in the extremely fast convergence speed of the algorithm. Besides, the Moore-Penrose (MP) generalized inverse is applied to determine the output weights. Last but not the least, there won't be a trouble of selecting termination criteria, determining learning rate or worrying over-tuned risk when using ELM instead of gradient learning algorithm.

Due to the extraordinary rapid speed and good accuracy of ELM, it has been widely employed in various fields. [104] applied kernel ELM as learning algorithm to predict the location of impact event on a clamped aluminum plate. The results were compared with other algorithms, such as SVM and BP. The comparison indicates the ELM has similar accuracy with SVM but is much faster than both SVM and BP. [105] employed ELM to design and construct stock price prediction model based on hidden information behind market news and stock price. The result shows that ELM achieves higher prediction accuracy and faster prediction speed than BP and RBF. [106] presented an ELM approach to multipath ultrasonic flowmeters measurement. The ELM is applied to 
determine the flow rate under mild and severe flow disturbances. The performance of ELM is compared with that of traditional integration methods on certain position as well as path orientation and the result shows that ELM outperforms the traditional methods in accuracy and efficiency. Also, ELM is insensitive to the installation effects of ultrasonic gas flowmeter. [107] adopted online sequential ELM to develop model predictive controller for air-ratio regulation based on different engine loads. The proposed method was conducted on a real engine for verification and the outcomes implied that it is a promising scheme for engine air-ratio control.

The single layer feedforward neural network (SLFN) is chosen for hybrid system modeling and the advantage of such network are 1) SLFN is an extensively used model which requires no prior knowledge; 2) its structure of single hidden layer is sufficient to achieve a balance between model accuracy and convergence speed; 3) it is flexible which benefits its further application towards control or optimization problem; 4) ELM can be directly adopted to train the SLFN network. The input weights are randomly given while the output weights are trained with batch learning in order to reduce computational time. Moreover, besides the normal constraint employed in the ELM, the requirement of cooling capacity and entrainment ratio is adopted as additional constraints in deriving the optimal output weights.

Since the method is settled down, another issue is raised, that is the selection of input parameters. Commonly, the input parameter selection is based on users' own expertise. However, inappropriate selection may cause lots of issues, such as the information loss of systems, the heavily computation cost or even the irrelative relationship with objectives. In order to systematically eliminate the unfeasible parameters of lesser importance index, the boosting tree algorithm is used to implement feature selection on raw input data set. The boosting tree shows its robust ability in the removal of irrelevant parameters. Minimization of overall regression error is set as criteria for each split at every node. The importance of each variable at each split is accumulated with the successive tree generating process. The variable with high importance means large contribution to the predicted output parameter.

To imply the preeminent performance of ELM, the modeling result of ELM will be used to compare with that trained with back propagation algorithm (BP), support vector 
machine (SVM) and radial basis function (RBF), respectively. It will be seen that the ELM model exhibits remarkable robustness against noises within experiment data and provides satisfying prediction of system states with lesser time consumed compared with other intelligent algorithm.

\subsection{System artificial neural network modeling}

\subsubsection{Feature selection}

As mentioned before, feature selection is necessary and helpful for neural modeling process since it can (1) simplify the model and makes them easier to interpret by users, (2) reduce the training times, (3) enhance the generalization of model by reducing over-fitting. Generally speaking, there are two kinds of selection pattern, namely filter method and wrapper method. Filter-based feature selection includes simple statistical test to determine if a feature is statistically significant. Feature may be assigned a scoring according to its importance and either be selected to be kept or removed from the dataset. The methods are often univariate and consider the feature independently, or with regard to the dependent variable [108, 109]. Example of some filter methods includes the Chi squared test, information gain and correlation coefficient scores. While, wrapper-based feature selection consider the selection of a set of features as a search problem, where different combinations are prepared, evaluated and compared to others. A predictive model is used to evaluate different combination of features and assign a score corresponding to their accuracy. This can achieve better performance but result in more computationally expensive. Some common wrapper methods are forward feature selection, backward feature selection and random forest.

In this research, in order to achieve a trade-off between accuracy and efficiency, boosting tree algorithm is employed to evaluate the importance of parameter waited for selection. But before this, principal component analysis (PCA) is conducted first to reduce the dimension of input feature. Based on multiplication between two covariance matrices, the ten input features are left for further selection. Boosting tree algorithm combines the multiple weak learners and builds a strong learner as a result. 
Given a distinct training pairs $\left\{\left(x_{1}, t_{1}\right),\left(x_{2}, t_{2}\right), \ldots\left(x_{\mathrm{n}}, t_{\mathrm{n}}\right)\right\}$ obtained from experiment, the goal is to find an approximation function $F^{*}(x)$ to the real function $F(x)$ that minimizes the value of loss function:

$$
F^{*}(x)=\underset{F(x)}{\arg \operatorname{minE}}{ }_{y, x}[L(y, F(x))]
$$

Boosting approaches the approximation through an additive expansion step:

$$
F(x)=\sum_{m=1}^{M} \beta_{m} h\left(x ; a_{m}\right)
$$

where $h(x ; a)$ are defined by parameters $a_{i}(i=1,2, \ldots, m)$ and $\beta$ are called expansion coefficients. Both $a_{i}$ and $\beta_{i}$ are determined through iteratively fit to the training data.

Firstly, set $F_{0}(x)$ as initial guess;

Then, for each $m=1,2, \ldots M$, we have

$$
\left(\beta_{m}, a_{m}\right)=\underset{\beta, a}{\arg \min } \sum_{i=1}^{N} L\left(y_{i}, F_{m-1}\left(x_{i}\right)+\beta h(x ; a)\right)
$$

and

$$
F_{m}(x)=F_{m-1}(x)+\beta_{m} h\left(x ; a_{m}\right)
$$

To solve equation (84), the least squares are used to fit the function $h(x ; a)$

$$
a_{m}=\underset{a}{\arg \min } \sum_{i=1}^{N}\left[\tilde{y}_{i m}-h(x ; a)\right]^{2}
$$

where $\tilde{y}_{i m}$ is the pseudo residuals expressed as

$$
\tilde{y}_{i m}=-\left[\frac{\partial L\left(y_{i}, F\left(x_{i}\right)\right)}{\partial F\left(x_{i}\right)}\right]_{F(x)=F_{m-1}(x)}
$$

Then the $\beta_{m}$ can be estimated as

$$
\beta_{m}=\arg \min \sum_{i=1}^{N} L\left(y_{i}, F_{m-1}\left(x_{i}\right)+\beta h\left(x_{i} ; a_{m}\right)\right)
$$

Therefore, the $\mathrm{K}$ terminal node regression trees can be represented as

$$
h\left(x ; R_{k m}\right)=\sum_{k=1}^{K} \bar{y}_{k m} h_{m}\left(x_{i}\right)
$$


With $\bar{y}_{k m}=$ mean $_{x_{i} \in R_{k m}}\left(\tilde{y}_{i m}\right)$, and equation (88) is turned into a prediction of a $\gamma_{k m}$ for each region $R_{k m}$

$$
\gamma_{k m}=\underset{\gamma}{\arg \min } \sum_{x_{i} \in R_{k m}} L\left(y_{i}, F_{m-1}\left(x_{i}\right)+\gamma h_{m}\left(x_{i}\right)\right)
$$

Thus, the approximation for real function $F$ in stage $m$ is

$$
F_{m}(x)=F_{m-1}(x)+\eta \gamma_{m} h(x)
$$

where $\eta$ is the learning rate of the procedure [110].

In this case, the learning rate is set to be 0.2 , and the total number of trees constructed is set as 150 .

Table 3 lists the result of parameter importance produced by boosting tree algorithm.

Table 3. Parameter importance

\begin{tabular}{|l|c|}
\hline Parameter & Importance \\
\hline Compressor frequency $f_{\text {com }}$ & 100 \\
\hline Condenser fan frequency $f_{\text {con }}$ & 79 \\
\hline Evaporator fan frequency $f_{\text {eva }}$ & 72 \\
\hline EEV opening percentage $A_{v}$ & 64 \\
\hline Condenser inlet pressure $P_{\text {con, in }}$ & 58 \\
\hline Ejector primary inlet pressure $P_{e, p \text { in }}$ & 51 \\
\hline Evaporator inlet temperature $T_{\text {eva, in }}$ & 44 \\
\hline Pump frequency $f_{\text {pump }}$ & 42 \\
\hline Ejector secondary inlet pressure $P_{e, \text { s in }}$ & 42 \\
\hline Pump inlet temperature $T_{\text {pump, in }}$ & 37 \\
\hline Generator inlet pressure $P_{\text {gen, in }}$ & 31 \\
\hline
\end{tabular}

Thus, six parameters with importance larger than 50 are selected to build the system model. 


\subsubsection{Model derivation}

Given $N$ distinct input data vectors $x_{i}$ and their corresponding target output $t_{i}$ that are obtained from experimental measurements of a hybrid ejector refrigeration cycle. The ith input pattern vector $X_{i}$ is defined as:

$$
\begin{aligned}
X_{i} & =\left[\begin{array}{lllll}
x_{i 1} & x_{i 2} & \cdots & x_{i 6}
\end{array}\right]^{T} \\
& =\left[\begin{array}{lllllll}
f_{c o m, i} & f_{\text {con }, i} & f_{\text {eva }, i} & A_{v, i} & P_{c o n, i n, i} & P_{e, p i n, i}
\end{array}\right]^{T}
\end{aligned}
$$

and the related $i$ th output vector $t_{i}$ is:

$$
t_{i}=\left[\begin{array}{llll}
t_{i 1} & t_{i 2} & t_{i 3} & t_{i 4}
\end{array}\right]^{T}=\left[\begin{array}{llll}
T_{\text {eva,out }, i} & T_{\text {con }, \text { out }, i} & P_{\text {eva }, \text { in }, i} & W_{\text {total }, i}
\end{array}\right]^{T}
$$

for $i=1,2, \cdots N$

To build a relationship between input vectors $x_{i}$ and output vector $t_{i}$, the structure of SLFN used is shown in Figure 62 as:

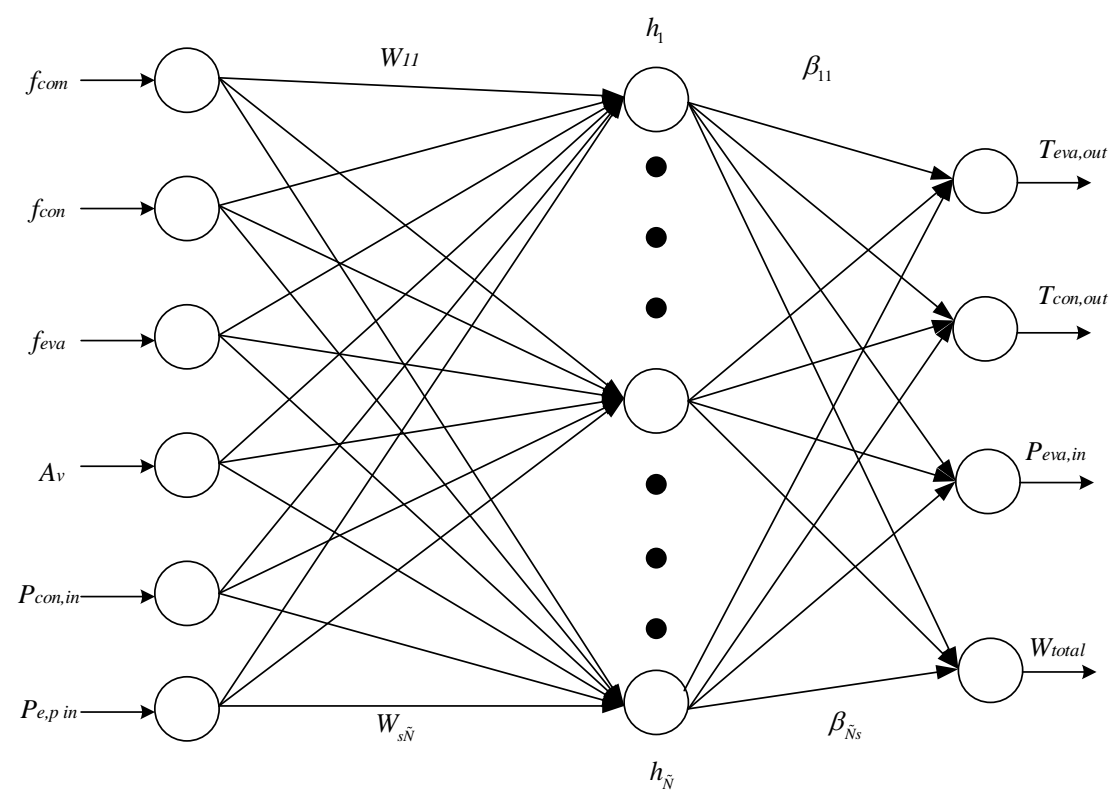

Figure 62 The SLFN of hybrid system model

where the nodes are linear in the input and output layer while are nonlinear in hidden layer with the nonlinear activation functions, described by

$$
h_{k i}=\varphi\left(W_{k}^{T} x_{i}\right)
$$


and $W_{i k}$ represents the input weight vector which connects the $i$ th input neuron to the $k$ th hidden neuron, defined as:

$$
W_{i k}=\left[\begin{array}{llll}
W_{1 k} & W_{2 k} & \cdots & W_{6 k}
\end{array}\right]^{T}
$$

for $k=1,2, \cdots \tilde{N}$, and according to idea of ELM, the value of input weights in $W_{k}$ are all randomly assigned.

The hidden layer vector $H_{i}$, corresponding to the input vector $X_{i}$, can then be shown as:

$$
\begin{aligned}
H_{i} & =\left[\begin{array}{llll}
h_{1 i} & h_{2 i} & \cdots & h_{\tilde{N} i}
\end{array}\right]^{T} \\
& =\left[\begin{array}{llll}
\varphi\left(W_{1}^{T} X_{i}\right) & \varphi\left(W_{2}^{T} X_{i}\right) & \cdots & \varphi\left(W_{\tilde{N}}^{T} X_{i}\right)
\end{array}\right]^{T}
\end{aligned}
$$

Therefore, the $j$ th output of the network can be obtained and expressed as:

$$
O_{j i}=\sum_{l=1}^{\tilde{N}} \beta_{l j} \varphi\left(W_{l}^{T} X_{i}\right) \quad j=1,2, \cdots 4
$$

Then the matrix form of all $N$ output vectors of the SLFN, which corresponding to the relative $N$ distinct input vectors $X_{1}, X_{2} \cdots X_{N}$, can be written as:

$$
\begin{aligned}
& O=\left[\begin{array}{ll}
O_{1} & O_{2} \cdots O_{N}
\end{array}\right] \\
& =\left[\begin{array}{cccc}
\sum_{l=1}^{\tilde{N}} \beta_{l 1} \varphi\left(W_{l}^{T} X_{1}\right) & \sum_{l=1}^{\tilde{N}} \beta_{l 1} \varphi\left(W_{l}^{T} X_{2}\right) & \ldots & \sum_{l=1}^{\tilde{N}} \beta_{l 1} \varphi\left(W_{l}^{T} X_{N}\right) \\
\vdots & \vdots & \ldots & \vdots \\
\sum_{l=1}^{\tilde{N}} \beta_{l 4} \varphi\left(W_{l}^{T} X_{1}\right) & \sum_{l=1}^{\tilde{N}} \beta_{l 4} \varphi\left(W_{l}^{T} X_{2}\right) & \ldots & \sum_{l=1}^{\tilde{N}} \beta_{l 4} \varphi\left(W_{l}^{T} X_{N}\right)
\end{array}\right] \\
& =\left[\begin{array}{cccc}
\beta_{1}^{T} \phi_{1} & \beta_{1}^{T} \phi_{2} & \cdots & \beta_{1}^{T} \phi_{N} \\
\beta_{2}^{T} \phi_{1} & \beta_{2}^{T} \phi_{2} & \cdots & \beta_{2}^{T} \phi_{N} \\
\vdots & \vdots & \vdots & \vdots \\
\beta_{4}^{T} \phi_{1} & \beta_{4}^{T} \phi_{2} & \cdots & \beta_{4}^{T} \phi_{N}
\end{array}\right]=\beta^{T} \phi
\end{aligned}
$$

with

$$
\begin{gathered}
\phi_{i}=\left[\begin{array}{cccc}
\varphi\left(W_{1}^{T} X_{i}\right) & \varphi\left(W_{2}^{T} X_{i}\right) & \cdots & \varphi\left(W_{\tilde{N}}^{T} X_{i}\right)
\end{array}\right]^{T} \\
\beta=\left[\begin{array}{llll}
\beta_{1} & \beta_{2} & \cdots & \beta_{4}
\end{array}\right]
\end{gathered}
$$




$$
\phi=\left[\begin{array}{llll}
\phi_{1} & \phi_{2} & \cdots & \phi_{N}
\end{array}\right]
$$

It can be seen that the performance of proposed SLFN network largely depends on the assignment of output weights since the input weights are randomly initialized. Thus, in order to obtain the optimal value of output weights, the following optimization problem is formulated to help determine the output weight matrix $\beta$ :

$$
\min \left\{\frac{1}{2}\|\varepsilon\|^{2}+\frac{d}{2}\|\beta\|^{2}\right\}
$$

where is the residual and expressed as

$$
=O \quad T={ }^{T} \quad T
$$

and subject to the equality constraint

$$
Q_{e i}=Q_{d e, i}=f\left(T_{o u t, i}, T_{i n, i}\right) \quad i=1,2, \cdots N
$$

where equation (104) indicates that the system cooling capacity must satisfy the demand quantity as well as obtain the optimization solution. The cooling load can be represented as a function of indoor and ambient temperature.

For the proposed system, the $Q_{e i}$ can be expressed as:

$$
\begin{aligned}
& \dot{Q_{e l}}=\dot{m}_{s} \times\left(H_{\text {eva,out }}-H_{\text {con,out }}\right) \\
& =\omega \times \dot{m}_{p}\left(a_{\text {eva }, 1}+a_{\text {eva }, 2} P_{\text {eva, in }}+a_{\text {eva }, 3} T_{\text {eva }, \text { out }}-\left(a_{c o n, 1}+a_{c o n, 2} P_{c o n, i n}+a_{c o n, 3} T_{c o n, o u t}\right)\right) \\
& =\omega \times f\left(T_{\text {con, out }}, W_{\text {pump }}\right) \times \\
& \left(a_{\text {eva }, 1}+a_{\text {eva }, 2} P_{\text {eva, in }}+a_{\text {eva }, 3} T_{\text {eva }, \text { out }}-\left(a_{c o n, 1}+a_{c o n, 2} P_{c o n, \text { in }}+a_{c o n, 3} T_{c o n, o u t}\right)\right)
\end{aligned}
$$

The above relation stands since the enthalpies at evaporator outlet and inlet can be calculated by linear fitting with related pressure and temperature.

For simplification purpose, let

$$
\gamma_{i}=\omega_{i} \times f\left(T_{\text {con }, \text { out }, i}, W_{\text {pump }, i}\right)
$$

After rearrangement of $Q_{e i}$, it can be expressed as follows: 


$$
\begin{aligned}
& \dot{Q}_{e i}=\gamma_{i}\left[\left(\left(a_{e v a, 1}-a_{c o n, 1}\right)+a_{\text {eva }, 2} T_{\text {eva,out }, i}-a_{c o n, 2} T_{c o n, o u t, i}+a_{e v a, 3} P_{e v a, i n, i}+a_{c o n, 3} P_{c o n, i n, i}\right)\right. \\
& =d_{0 i}+\left[\begin{array}{llll}
d_{1 i} & d_{2 i} & d_{3 i} & 0
\end{array}\right]\left[\begin{array}{c}
T_{\text {eva }, \text { out }, i} \\
T_{\text {con,out }, i} \\
P_{\text {eva }, i n, i} \\
W_{\text {total }, i}
\end{array}\right] \\
& =d_{0 i}+d_{i}^{T} O_{i}
\end{aligned}
$$

where

$$
\left\{\begin{array}{c}
d_{0 i}=\gamma_{i}\left(a_{e v a, 1}-a_{c o n, 1}+a_{c o n, 3} P_{c o n, i n, i}\right) \\
d_{1 i}=\gamma_{i} a_{e v a, 2} \\
d_{2 i}=-\gamma_{i} a_{c o n, 2} \\
d_{3 i}=\gamma a_{e v a, 3} \\
d_{4 i}=0
\end{array}\right.
$$

Then, the Lagrange multiplier is applied for minimization with equality constraint problem, and the following function is constructed:

$$
\begin{aligned}
L= & \frac{1}{2} \sum_{i=1}^{5} \sum_{j=1}^{N} \varepsilon_{i j}^{2}+\frac{d}{2} \sum_{i=1}^{5} \sum_{j=1}^{\tilde{N}} \beta_{i j}^{2}+ \\
& \sum_{i=1}^{5} \sum_{j=1}^{N} \lambda_{i j}\left(\beta_{i}^{T} \phi_{j}-t_{i j}-\varepsilon_{i j}\right)+\sum_{j=1}^{N} \bar{\lambda}_{j}\left(Q_{e j}-f\left(T_{o u t, j}, T_{i n, j}\right)\right)
\end{aligned}
$$

Then substituting equation (107) into the right most part of above function, after rearrangement, it can be obtained that

$$
\begin{aligned}
& \sum_{j=1}^{N} \bar{\lambda}_{j}\left(Q_{e j}-Q_{d e j}\right)=\sum_{j=1}^{N} \bar{\lambda}_{j}\left(d_{0 j}+d_{j}^{T} O_{j}-f\left(T_{\text {out }, \mathrm{j}}, T_{i n, j}\right)\right) \\
& =\bar{\lambda}^{T} d_{0}+\sum_{j=1}^{N} \bar{\lambda}_{j} d_{j}^{T} O_{j}-\bar{\lambda}^{T} f\left(T_{\text {out }}, T_{\text {in }}\right) \\
& =\left\{\begin{array}{l}
\bar{\lambda}^{T} d_{0}+\sum_{j=1}^{N} \bar{\lambda}_{j} \sum_{i=1}^{5} d_{i j} \beta_{i}^{T} \phi_{j}-\bar{\lambda}^{T} f\left(T_{\text {out }}, T_{\text {in }}\right) \\
\bar{\lambda}^{T} d_{0}+\sum_{j=1}^{N} \bar{\lambda}_{j} \sum_{i=1}^{5} d_{i j}\left(\varepsilon_{i j}+t_{i j}\right)-\bar{\lambda}^{T} f\left(T_{\text {out }}, T_{\text {in }}\right)
\end{array}\right.
\end{aligned}
$$

There are two kinds of expression since the output of the network can be expressed as either the result of hidden layer output multiplied by output weights or the result of desired output amended by error. 
Then substitute both expressions into Lagrange function and take the derivative with respect to $\beta_{i j}$ for the first expression and $\varepsilon_{i j}$ for the second one.

It can be obtained that

$$
\begin{aligned}
\frac{\partial L}{\partial \beta_{i j}} & =d \beta_{i j}+\sum_{j=1}^{N} \lambda_{i j} \phi_{i j}+\sum_{j=1}^{N} d_{i j} \phi_{i j} \bar{\lambda}_{j} \\
& =d \beta_{i j}+\sum_{j=1}^{N} \phi_{i j}\left(\lambda_{i j}+\bar{\lambda}_{j} d_{i j}\right)=0
\end{aligned}
$$

And

$$
\frac{\partial L}{\partial \varepsilon_{i j}}=\varepsilon_{i j}+\lambda_{i j}+\bar{\lambda}_{j} d_{i j}=0
$$

By comparing the result, it can be found that

$$
d \beta=-\phi \varepsilon^{T}
$$

Replace the $\varepsilon$ with $\beta^{T} \phi$-T, and after rearrangement, the $\beta$ can be expressed as

$$
\beta=\left(\phi \phi^{T}-d I\right)^{-1} \phi T^{T}
$$

Therefore, the output weight estimation can be determined based on (114) and then be applied in network for the prediction of mentioned output vector in (93).

\subsection{Validation and discussion}

A SLFN with 6 input nodes, 50 hidden nodes and 4 output nodes is constructed for modeling the hybrid system proposed in order to predict the system states. The training and testing input data vectors are collected under different operation conditions. While the desired output data vectors of the SLFN, corresponding to all input data, are obtained from measurement via the pressure sensors, temperature sensors and power meter, respectively.

The proposed SLFNs are trained with BP, SVM, RBF and ELM, respectively for comparison purpose with the training data pairs measured under stable operation conditions. To evaluate the generalization performance after training, the SLFN models are tested with 800 groups of measured data.

The results together with discussion are illustrated and are shown in Figures 63-70. 


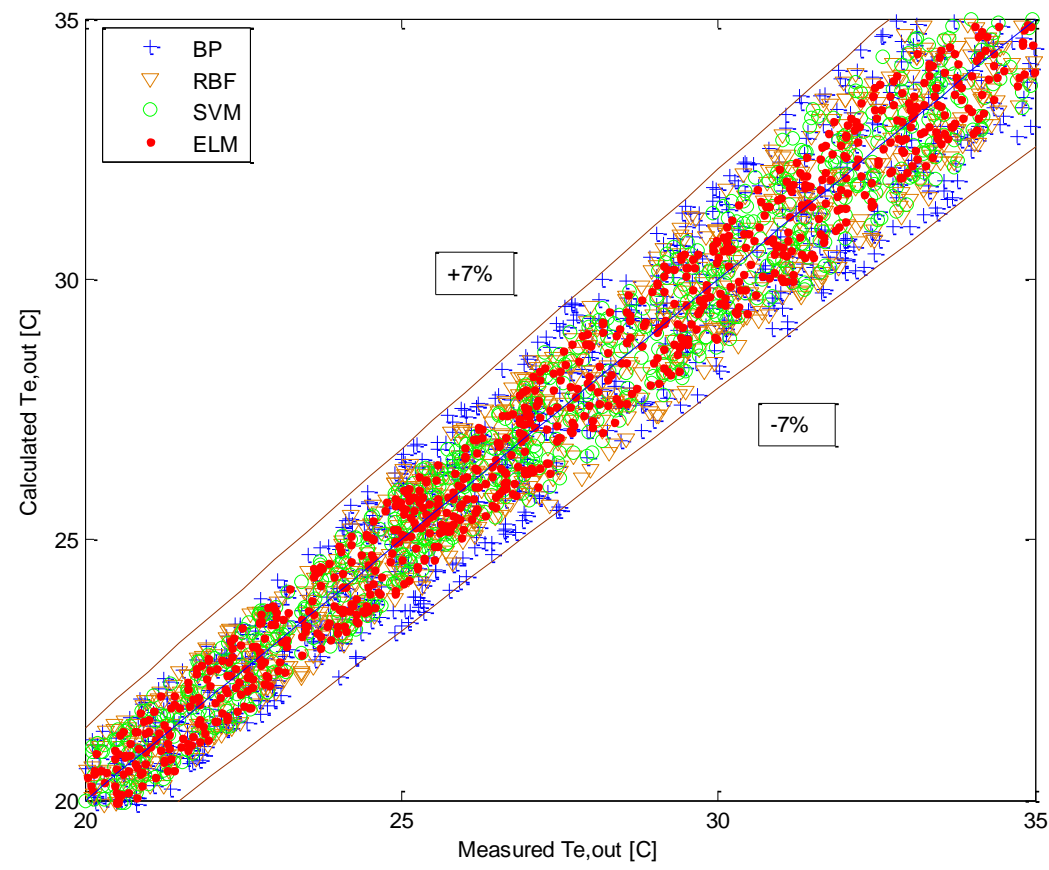

Figure 63 Training result of $T_{\text {eva,out }}$

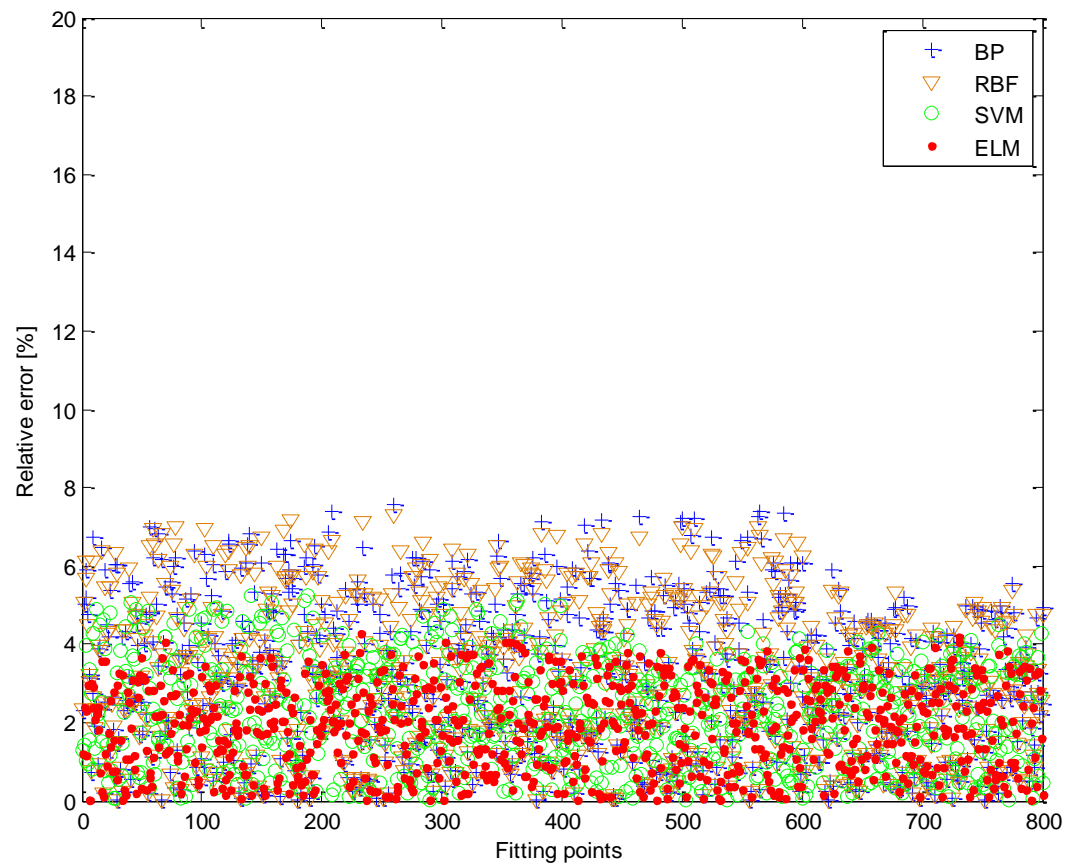

Figure 64 Relative error of $T_{\text {eva,out }}$ training set 


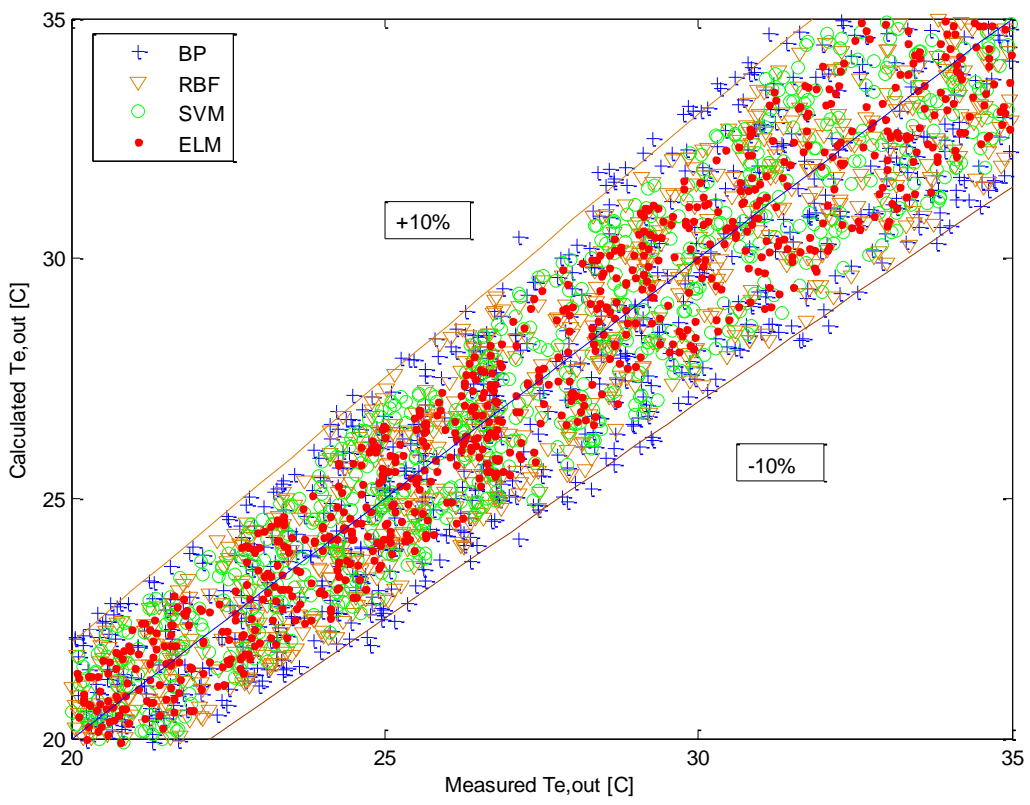

Figure 65 Testing result of $T_{\text {eva,out }}$

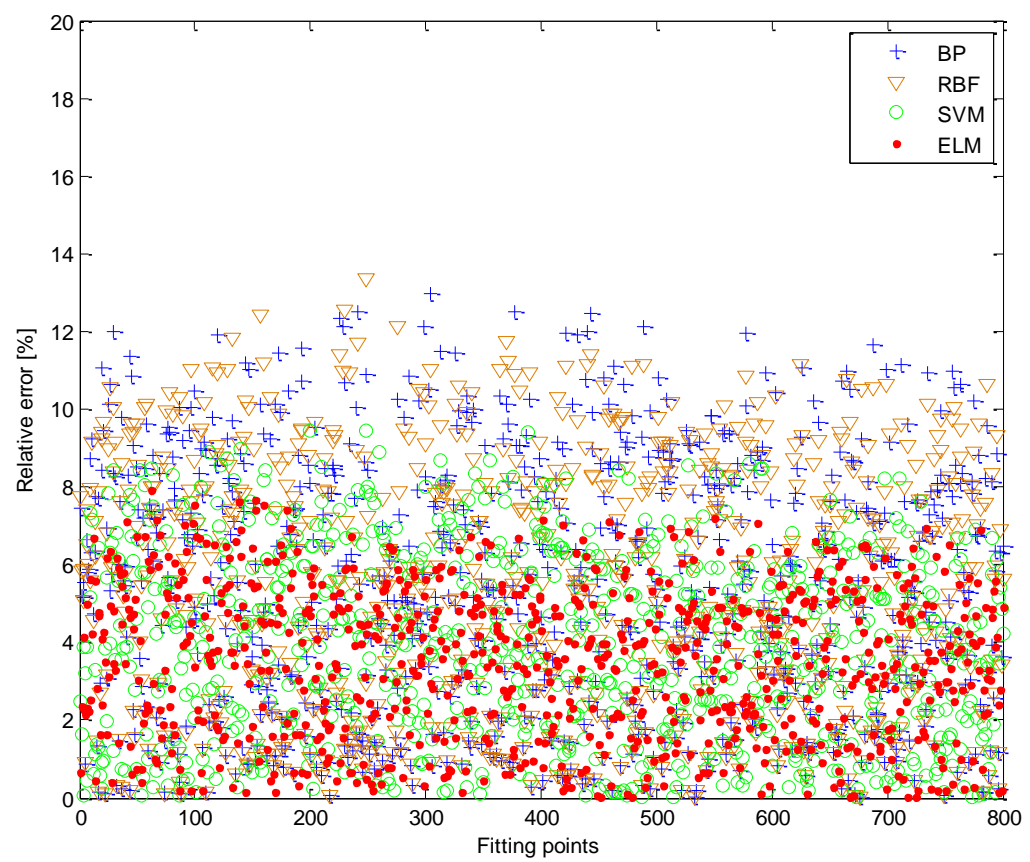

Figure 66 Relative error of $T_{\text {eva,out }}$ testing set 


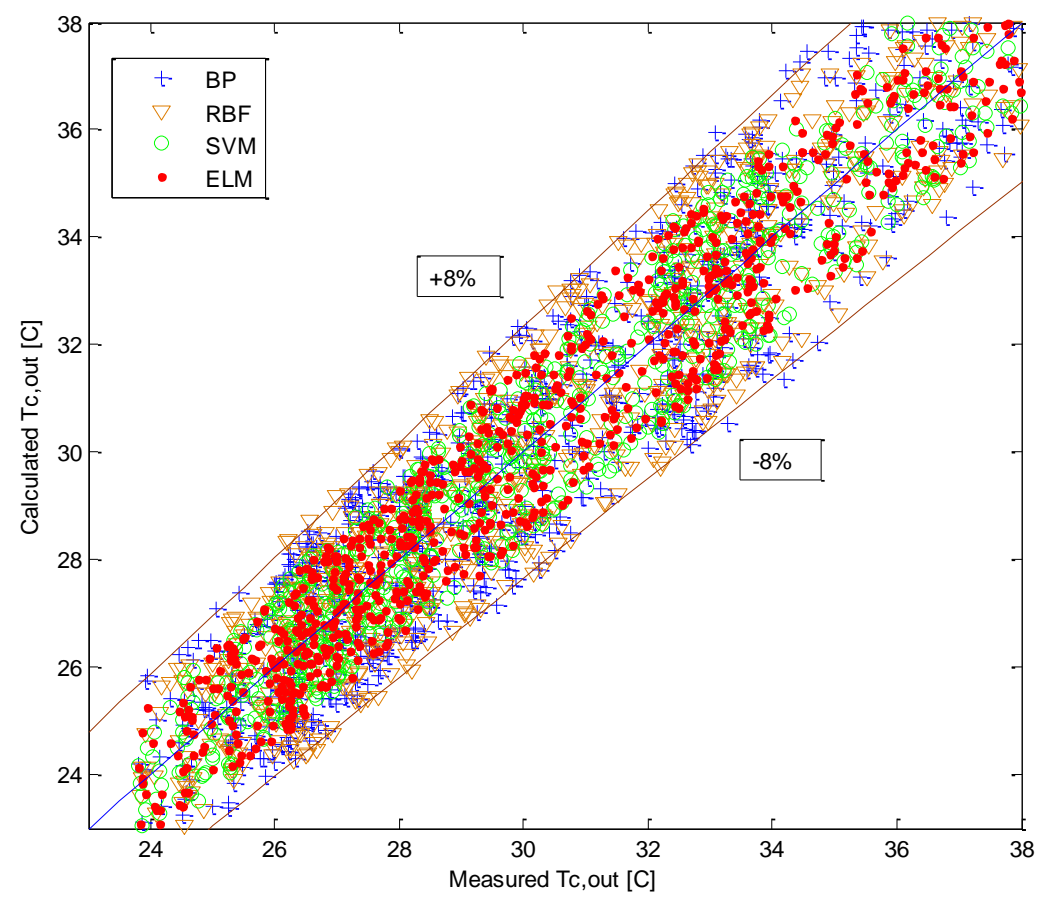

Figure 67 Training result of $T_{\text {con, out }}$

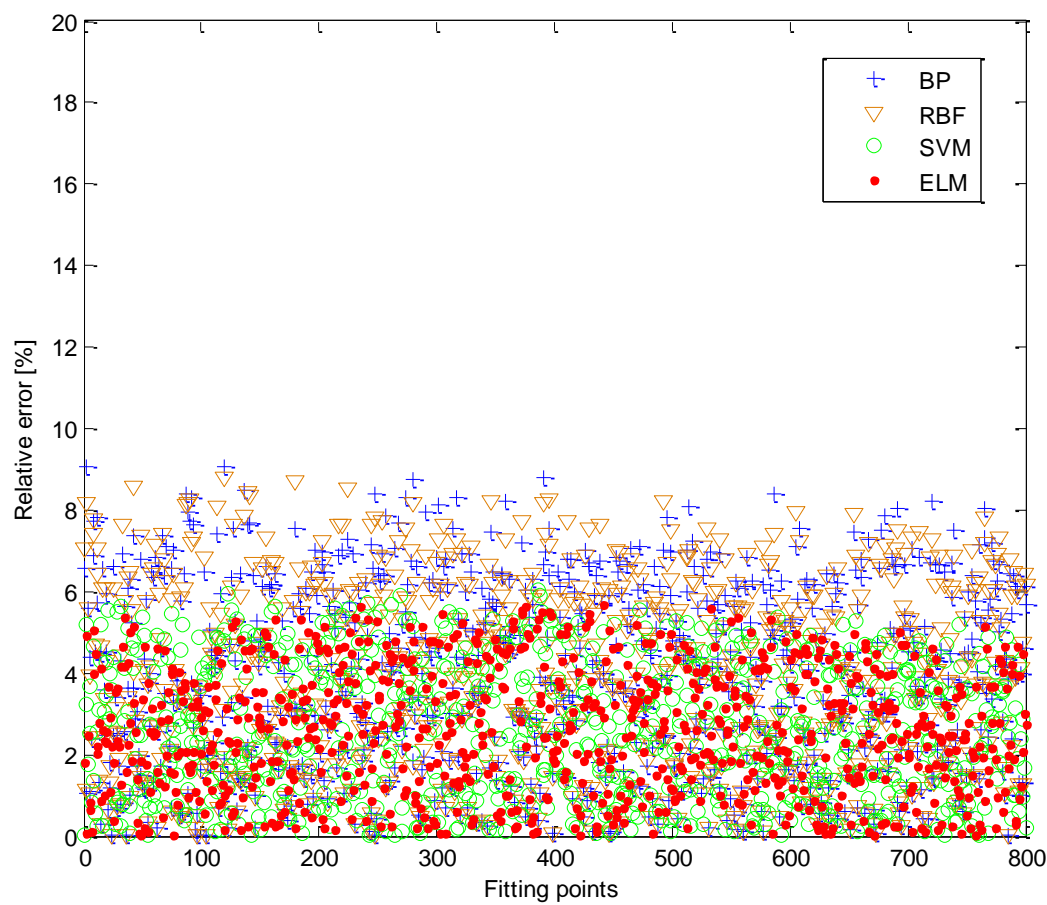

Figure 68 Relative error of $T_{\text {con,out }}$ training set 


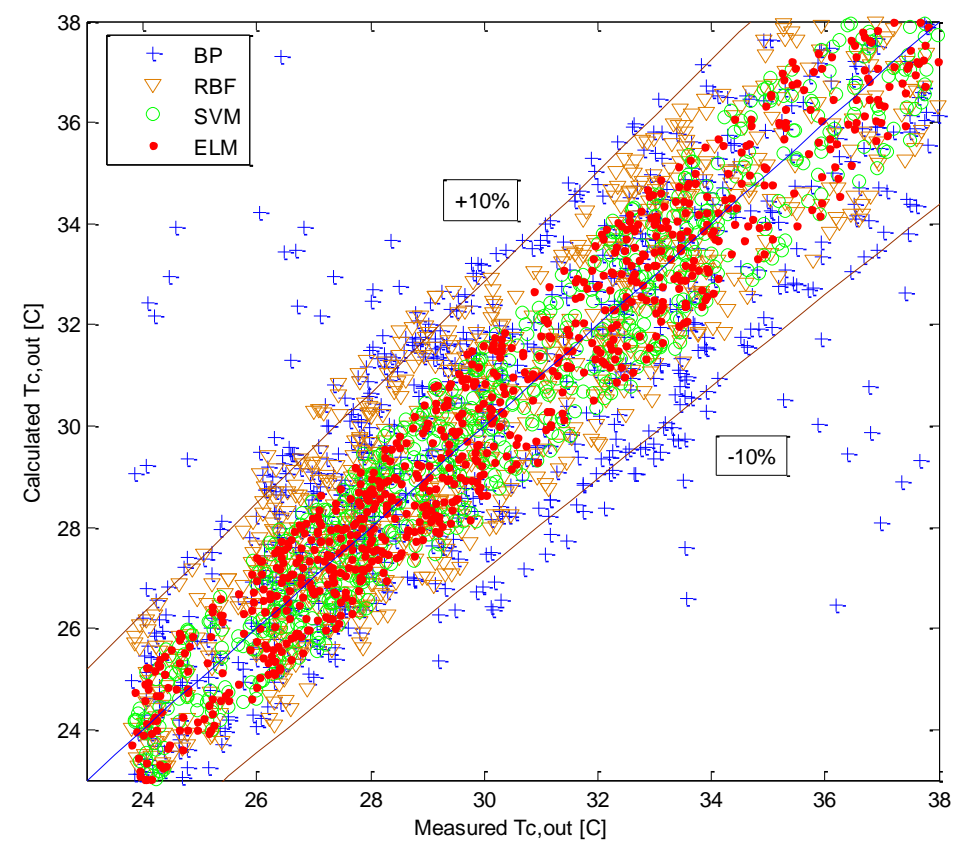

Figure 69 Testing result of $T_{\text {con,out }}$

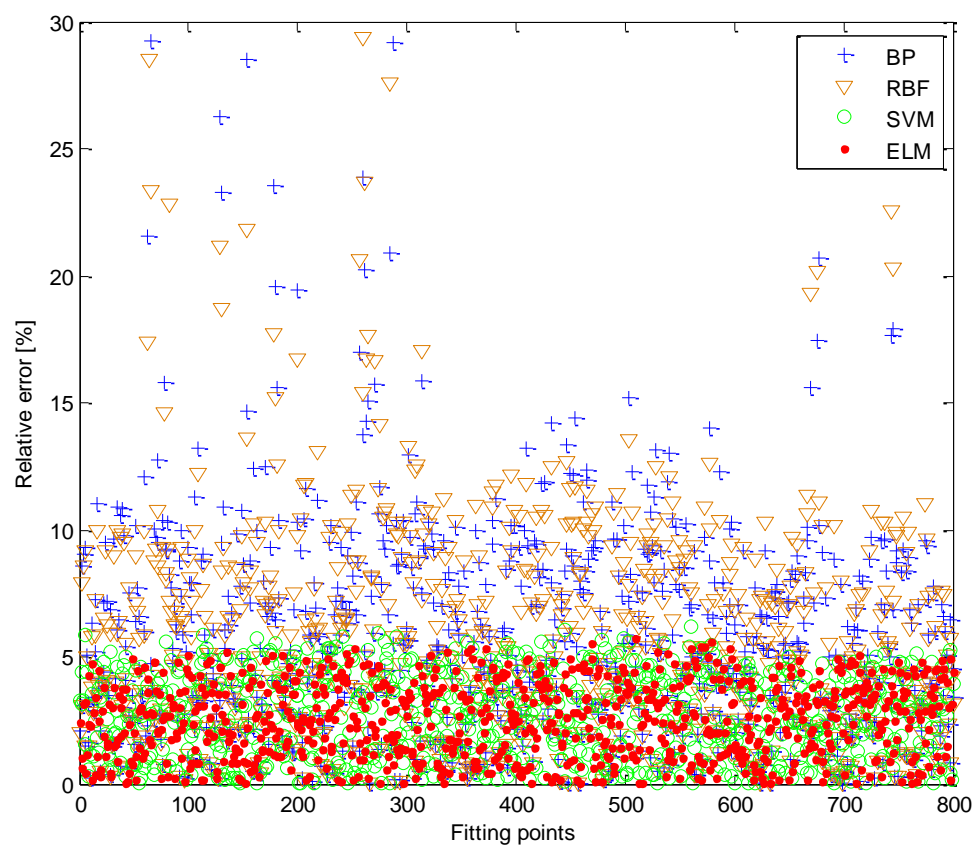

Figure 70 Relative error of $T_{\text {con,out }}$ testing set

Both the training and testing results for evaporating and condensing outlet temperature are depicted in Figure 63 to Figure 70, where the predicted values are provided by the SLFNs 
trained with the BP, the RBF, SVM and the ELM, respectively. It can be observed that the prediction deviations of these two temperatures obtained from the SLFN with the ELM are the smallest, the corresponding points are closer to the middle line. Its prediction errors of $T_{\text {eva,out }}$ and $T_{\text {con,out }}$ are almost within $\pm 6 \%$ and $\pm 5 \%$, respectively. The prediction errors of SVM, on the other hand, are within $\pm 8 \%$ and $\pm 5.5 \%$, respectively, which are slightly larger than that of ELM. It is also noticeable that the deviations of the predicted value obtained from the SLFN with the BP and RBF are much larger than the other two algorithms, the maximum deviation of evaporating and condensing temperature predictions can even reach $15 \%$ and $30 \%$, respectively.

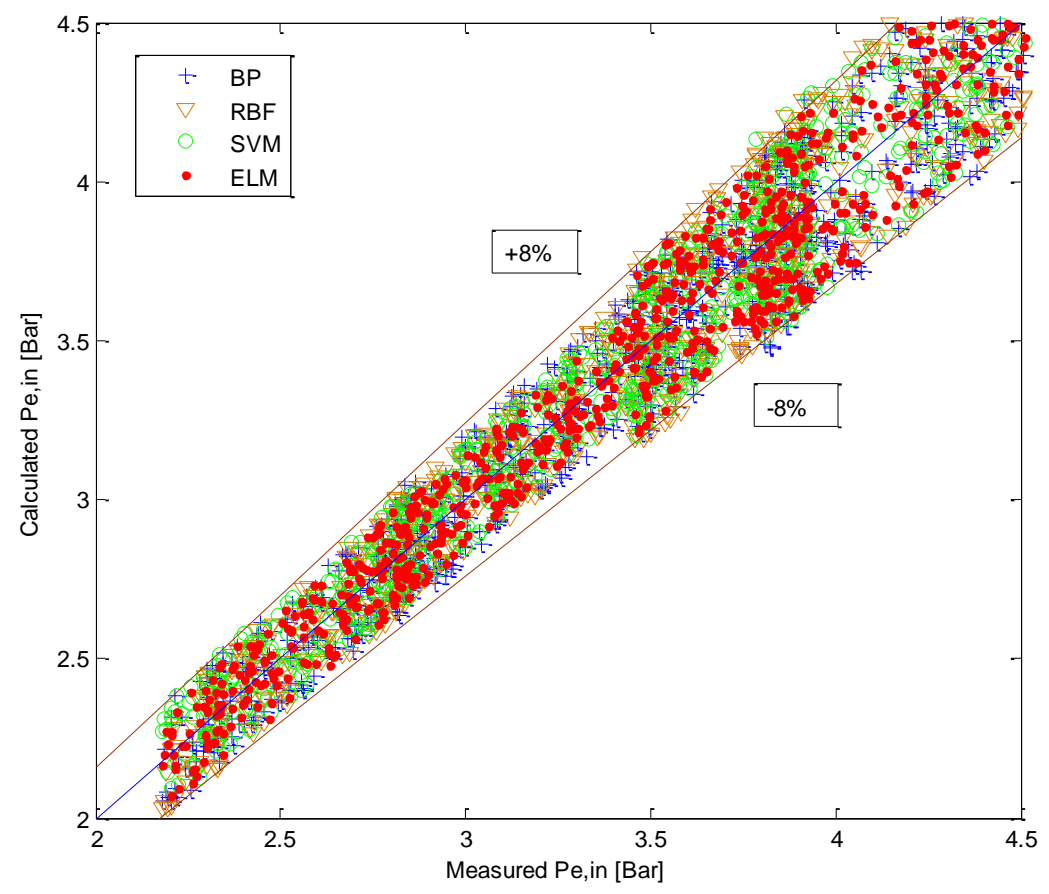

Figure 71 Training result of $P_{\text {eva,in }}$ 


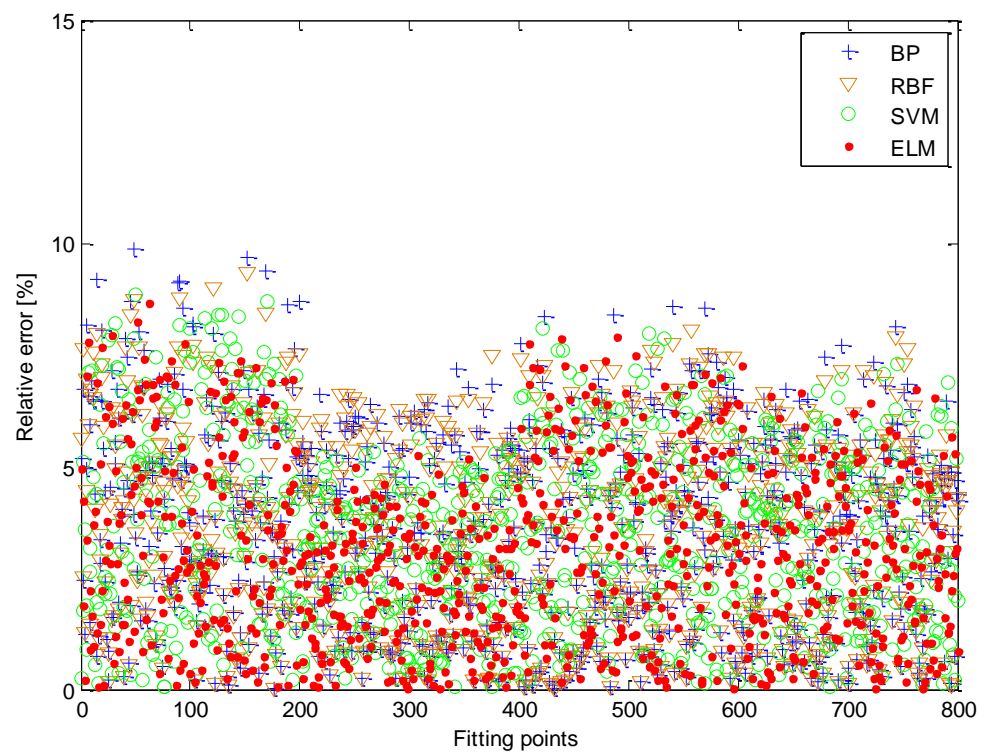

Figure 72 Relative error of $P_{\text {eva, in }}$ training set

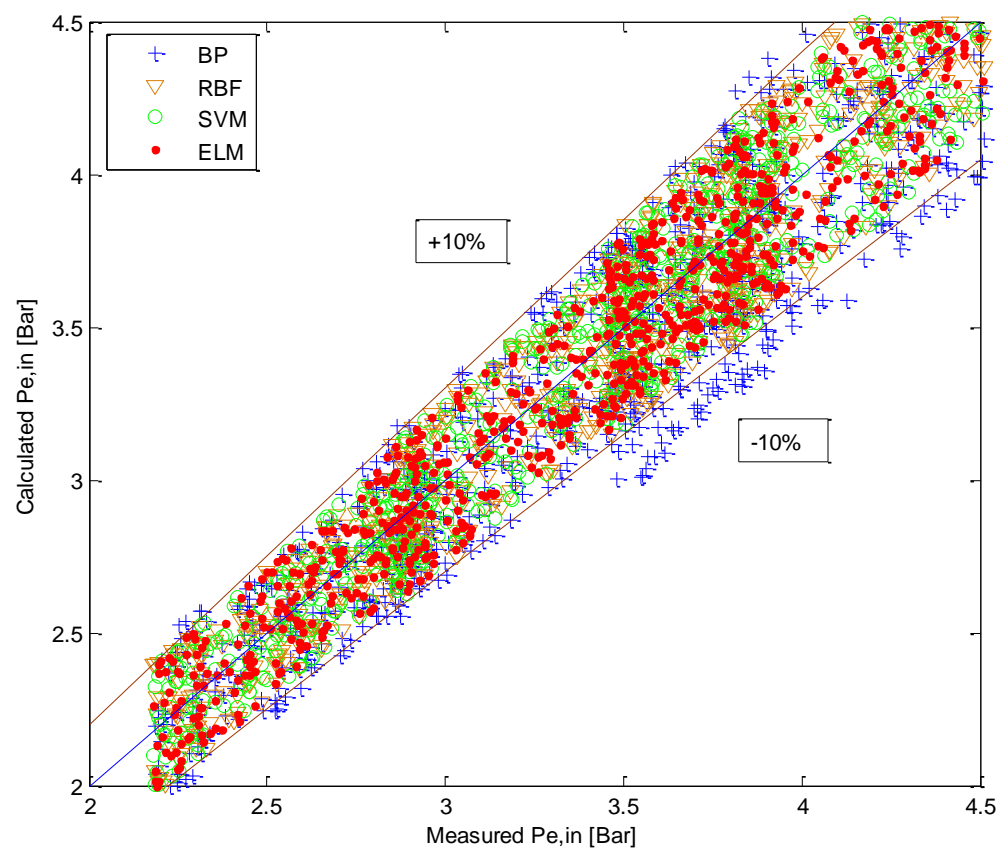

Figure 73 Testing result of $P_{\text {eva, in }}$ 


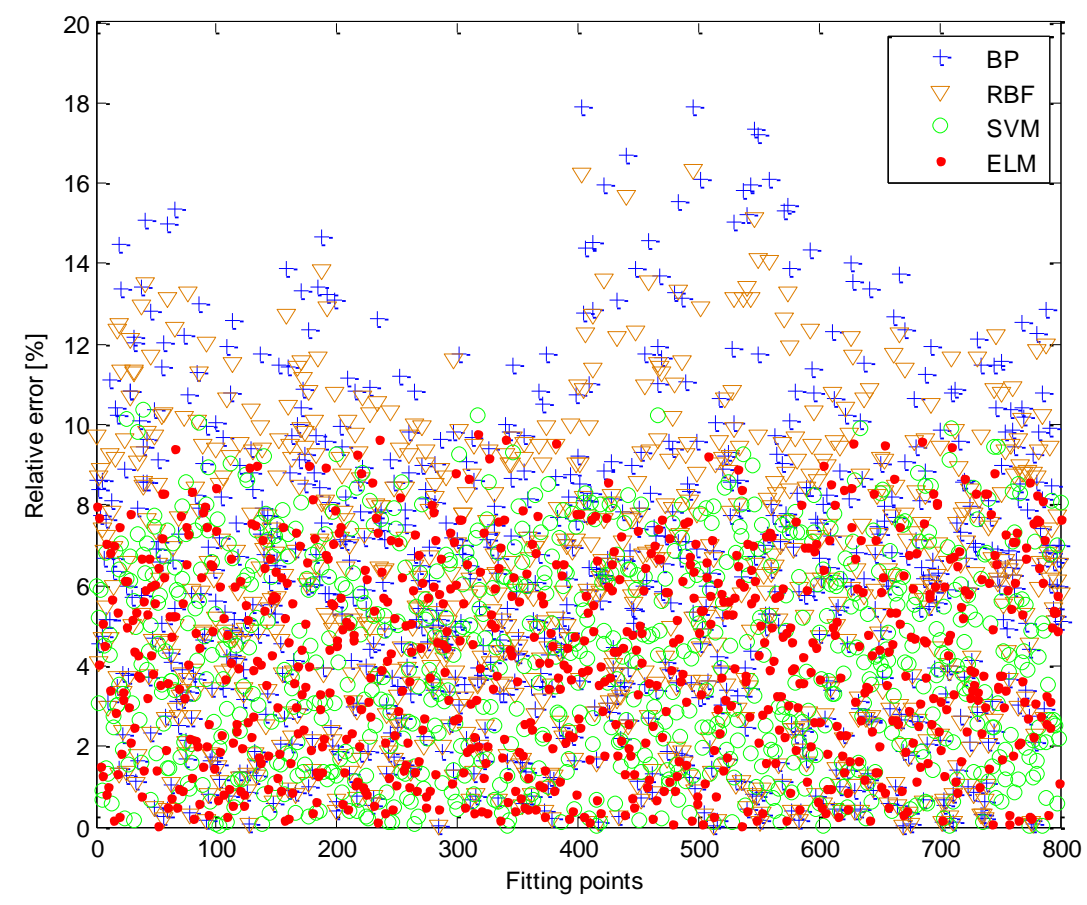

Figure 74 Relative error of $P_{\text {eva,in }}$ testing set

Figure 71 to Figure 74 show the training and testing performances of the evaporator inlet pressure with the SLFNs trained with the ELM, the RBF, the SVM and the BP, respectively. It has been seen that the prediction deviations of $P_{\text {eva,in }}$ obtained from the SLFN with the BP and RBF are larger than $12 \%$ and $10.5 \%$, respectively. Furthermore, these prediction errors tend to increase as evaporator pressure grows. However, the prediction errors of both the ELM and the SVM are within $\pm 8 \%$. Furthermore, the prediction error of SVM also shows the trend of growth for large evaporator pressure. In contrast, performances of ELM are stable in spite of a large testing range. It is also noticeable that the tested ELM consists of only 50 hidden nodes, considering that the advantage of ELM is its high speed for training large scale SLFN, if more hidden nodes are added, the prediction errors of ELM can be significantly reduced with a little more additional time. 


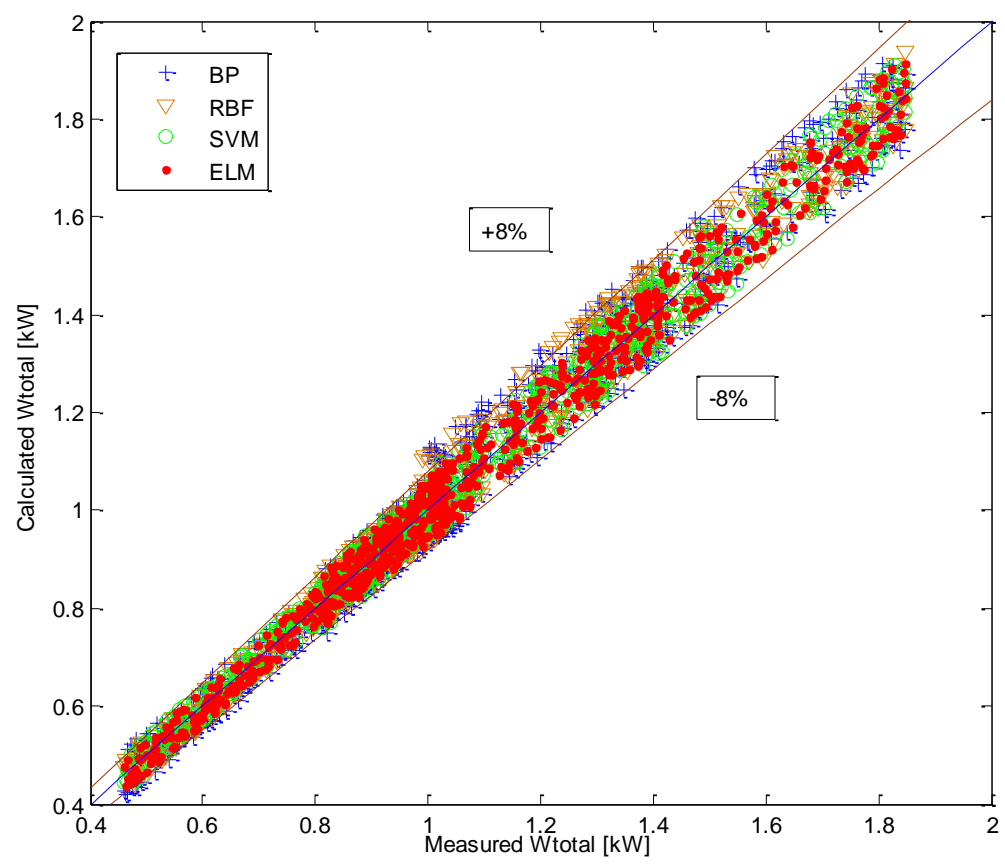

Figure 75 Training result of $W_{\text {total }}$

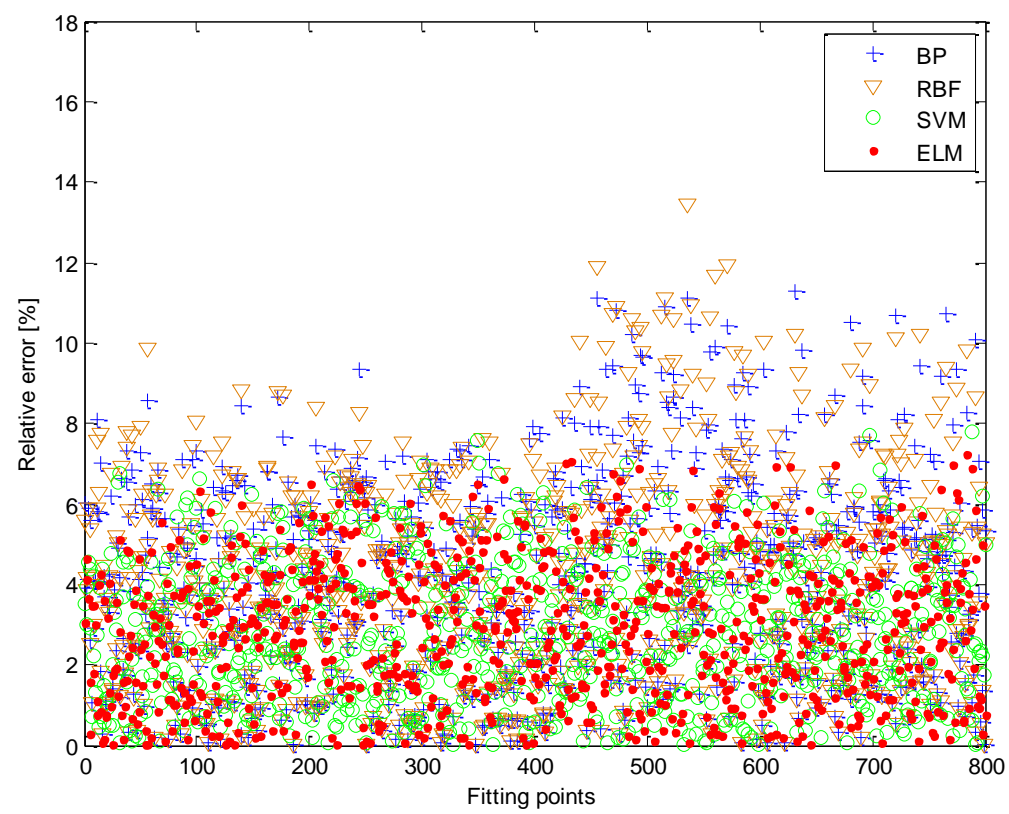

Figure 76 Relative error of $W_{\text {total }}$ training set 


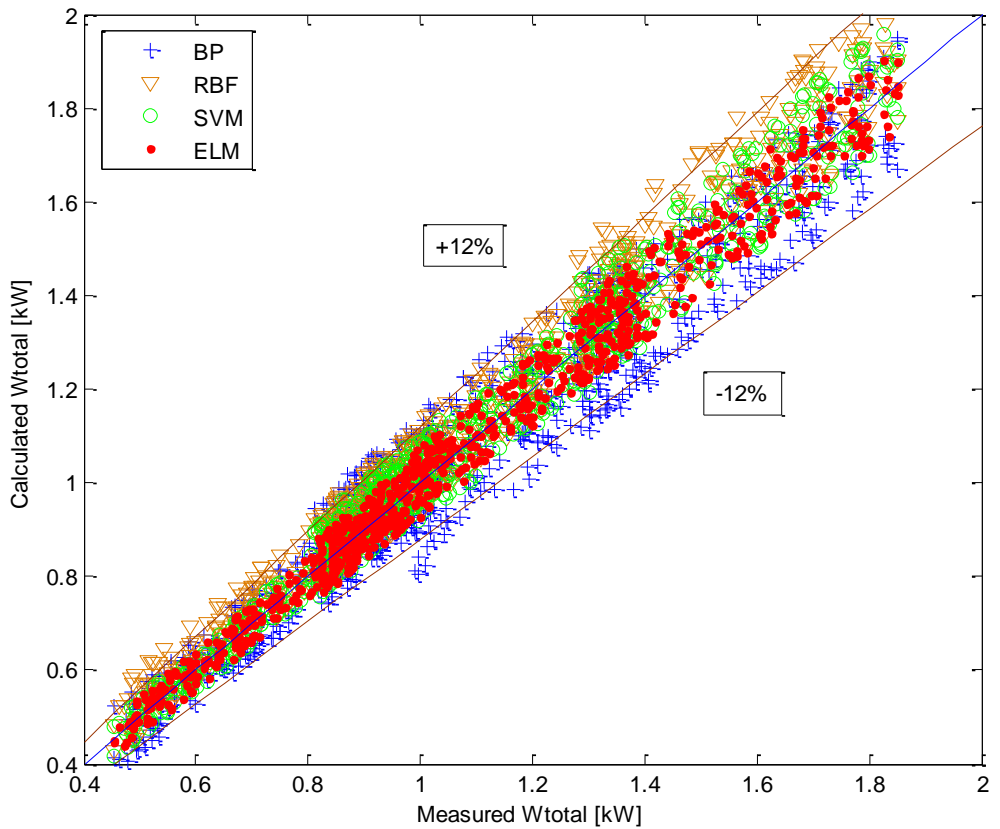

Figure 77 Testing result of $W_{\text {total }}$

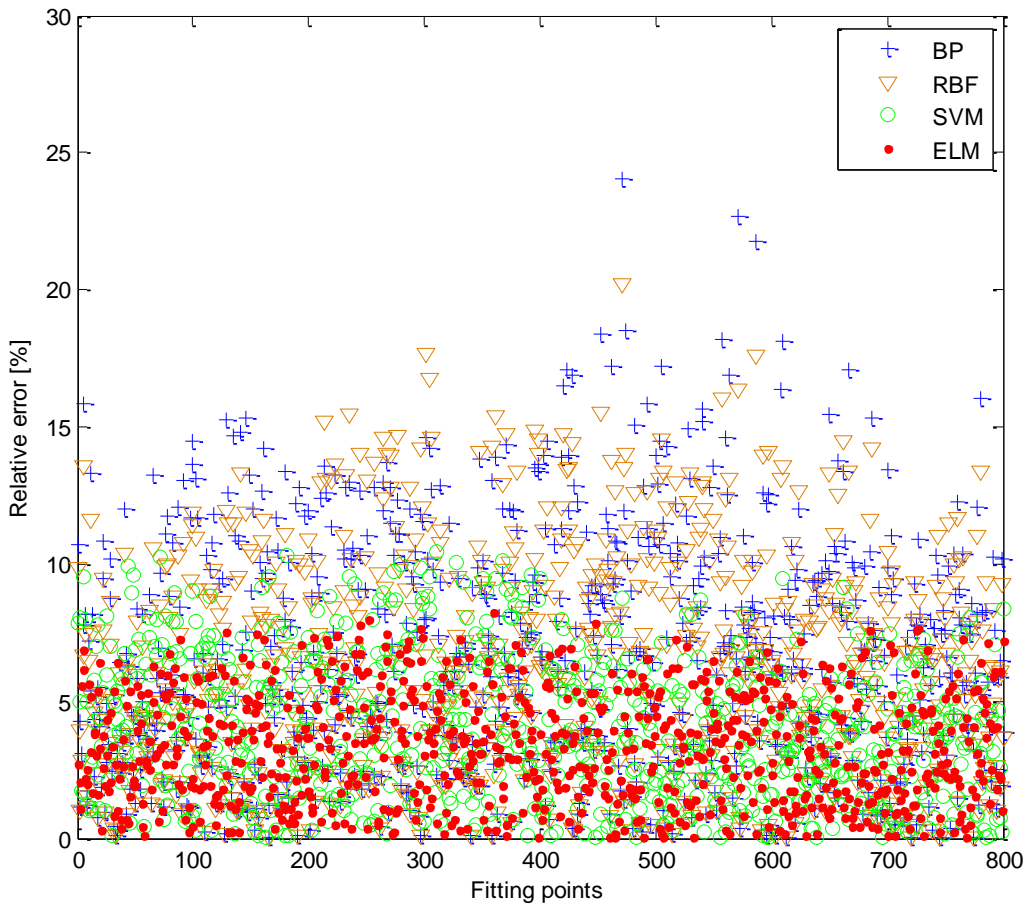

Figure 78 Relative error of $W_{\text {total }}$ testing set 
The total energy consumption, which is regarded as an output of the proposed model, aims at identifying the sum of the energy consumptions of liquid pump, compressor, condenser and evaporator fans. Due to the fact that the transient energy consumption cannot be measured by the power meter directly, and all the energy consumption devices have complex transient responses, the training and testing result of energy consumptions are all generated by using the hybrid model whose coefficients are determined with fitting the measured energy consumption data under the steady states.

It can be seen from Figure 75 to Figure 78 that the testing performance of the SLFN with $\mathrm{BP}$ is the least satisfying when compared with the ones trained with the RBF, the SVM and the ELM. Since gradient decent method is applied in BP to help determine the global minimum of weights, and the robustness improvement of trained weights is not considered, these all contribute to the poor performance of BP. Additionally, it can be easily trapped into some local minima during BP training process if there are a large number of hidden nodes in SLFN. It can be further revealed from the figures that, even though the performance of the SLFN with the RBF and the SVM are better than that of the BP, the SLFN trained with the ELM has the smallest average relative mean error due to the characteristic of global optimization with the consideration of improving the robustness with respect to the disturbances in the cost function as well as the proposed Lagrange function, compared the ones with the rest. 


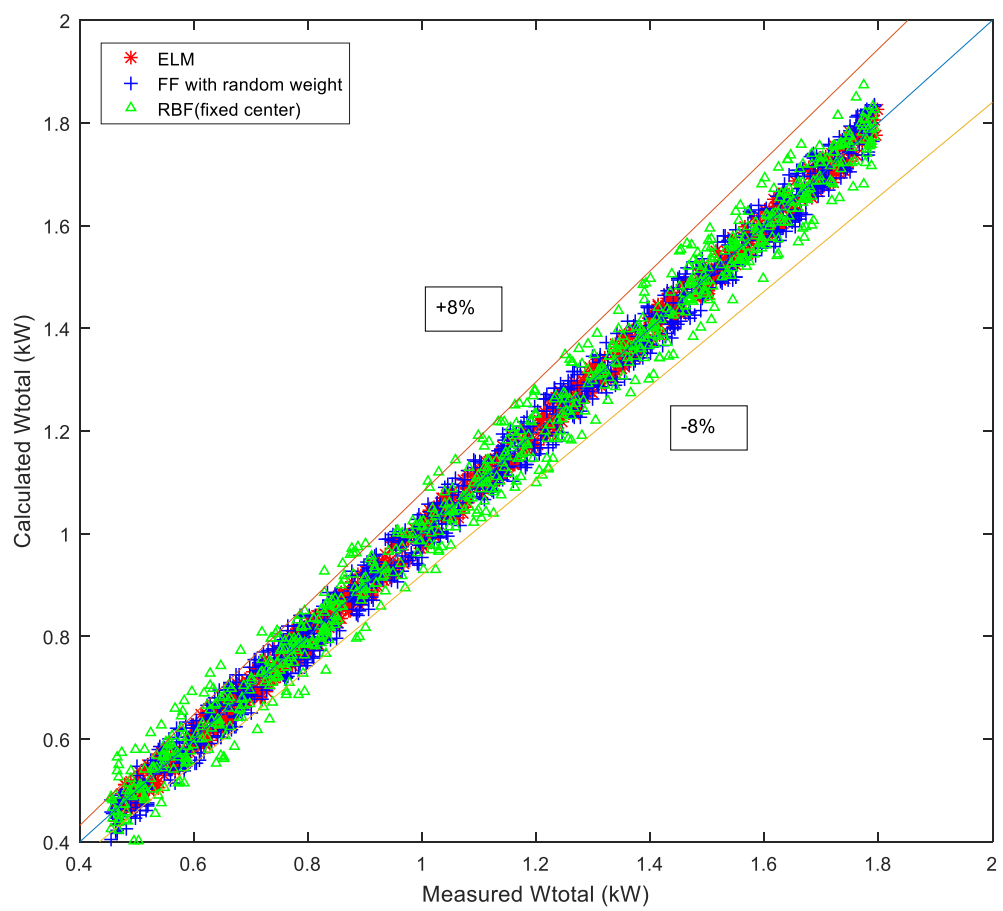

Figure 79 Further comparison training result of $W_{\text {total }}$

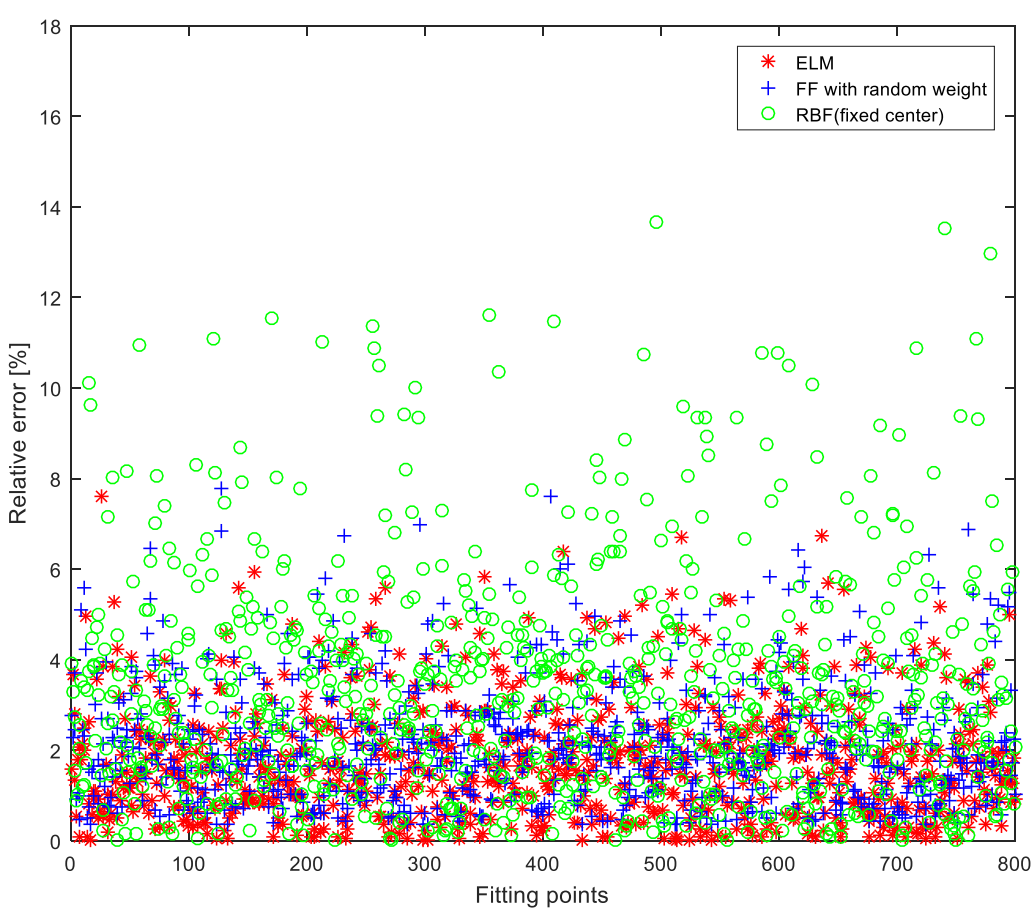

Figure 80 Further comparison relative error of $W_{\text {total }}$ training set 


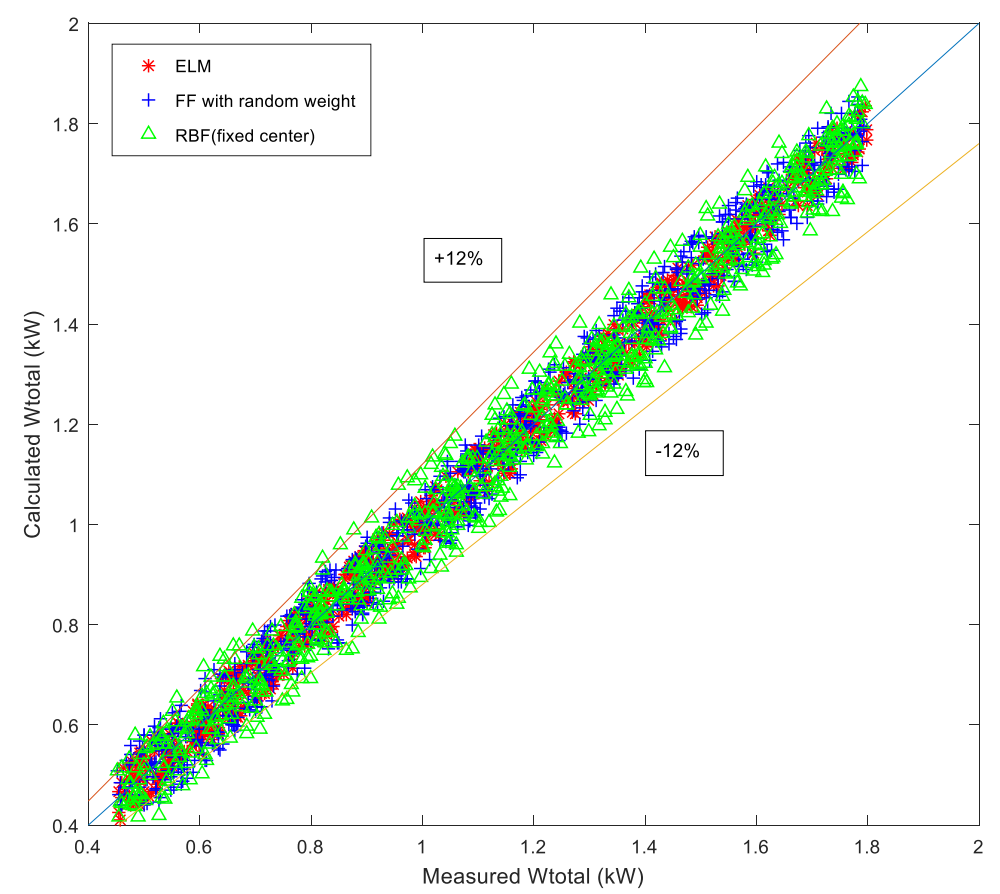

Figure 81 Further comparison testing result of $W_{\text {total }}$

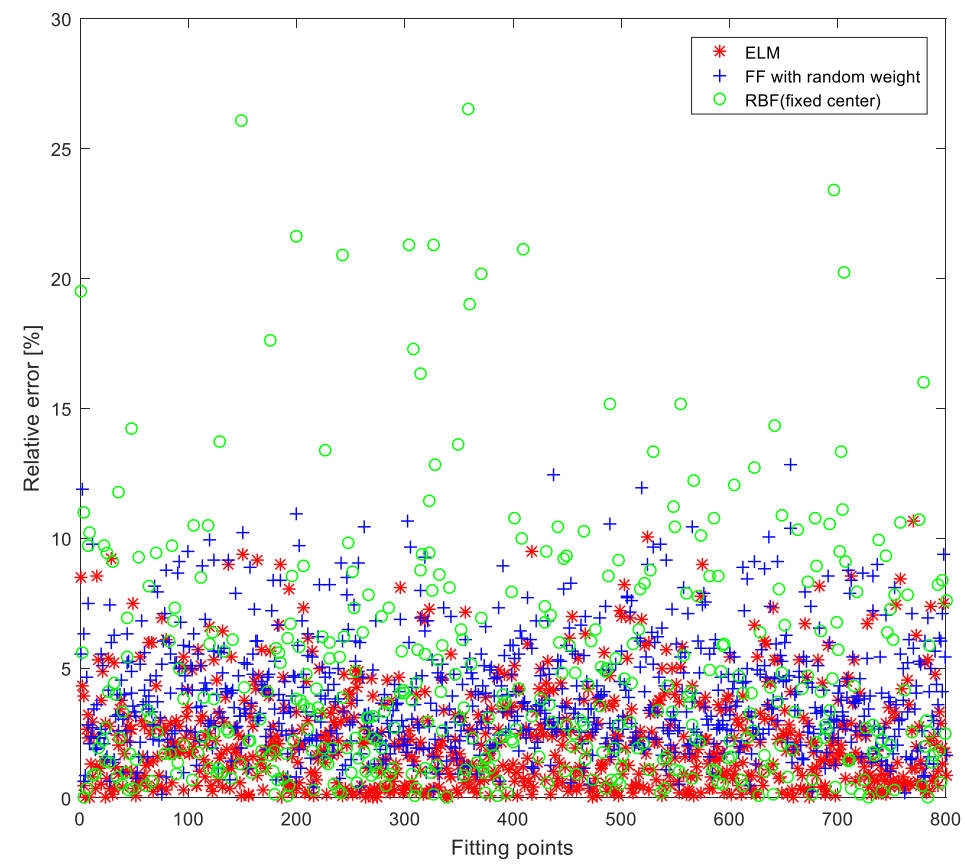

Figure 82 Further comparison relative error of $W_{\text {total }}$ testing set 
In order to better illustrate the out-performance of ELM, further comparison with another two networks, namely the RBF network with fixed center and the feedforward networks with random weights, is conducted. From the Figure 79 to Figure 82, it can be observed that the performance of fixed center RBF network has the least satisfying performance than the others. Additional, within the lower $W_{\text {total }}$ range, the ELM is more accurate than feedforward networks.

Moreover, the detailed comparison of testing accuracies of proposed SLFN trained with the BP, the RBF (both fixed and random centers), the Feed-forward with random weight, the SVM and ELM is shown specifically in Table 4. The mean squared error (MSE) and relative mean error (RME) are employed to evaluate the performance of the BP (with 50 hidden nodes), the RBF (with 50 hidden nodes and random Gaussian centers or fixed centers), the SVM (with $C=2^{5}$ and $\gamma=2^{-4}$ ), the Feed forward network with random weights and the ELM (with 300 hidden nodes), respectively. It can be seen that the SLFN with the $\mathrm{BP}$ consumes the longest training time due to its recursive updating of both the input and output weights during training process. While, on the contrast, the training time consumed by the SLFN with the ELM is the shortest. It can be easily interpreted that since there is no weights updating needed for the randomly selected input weights of the SLFN during the training, a significantly amount of time is saved.

Table 4. The comparison of testing accuracies of different methods

\begin{tabular}{|l|l|l|l|l|l|}
\hline \multicolumn{2}{|l|}{ Outputs } & $T_{\text {eva,out }}$ & $T_{\text {con,out }}$ & $P_{\text {eva, in }}$ & $W_{\text {total }}$ \\
\hline \multirow{2}{*}{ BP } & MSE & 0.8283 & 0.4352 & 0.3283 & 0.1294 \\
\cline { 2 - 6 } & RME & $4.93 \%$ & $3.64 \%$ & $6.17 \%$ & $9.68 \%$ \\
\hline \multirow{2}{*}{$\begin{array}{l}\text { RBF(random } \\
\text { center) }\end{array}$} & MSE & 0.6579 & 0.3779 & 0.2741 & 0.0760 \\
\cline { 2 - 6 } & RME & $4.27 \%$ & $3.16 \%$ & $5.46 \%$ & $6.35 \%$ \\
\hline \multirow{2}{*}{$\begin{array}{l}\text { RBF(fixed } \\
\text { center) }\end{array}$} & MSE & 0.6631 & 0.3815 & 0.2837 & 0.0844 \\
\cline { 2 - 6 } & RME & $4.33 \%$ & $3.27 \%$ & $5.61 \%$ & $6.57 \%$ \\
\hline \multirow{2}{*}{\begin{tabular}{l} 
Feed- $\begin{array}{l}\text { forward(random } \\
\text { weight) }\end{array}$ \\
\cline { 2 - 6 } SVM
\end{tabular}} & RSE & 0.534 & 0.3581 & 0.2589 & 0.0648 \\
\cline { 2 - 6 } & RME & $3.97 \%$ & $3.11 \%$ & $4.93 \%$ & $6.21 \%$ \\
\cline { 2 - 6 } & RME & 0.4302 & 0.3478 & 0.2374 & 0.0544 \\
\cline { 2 - 6 } & ELM & $3.86 \%$ & $2.92 \%$ & $4.58 \%$ & $5.88 \%$ \\
\hline
\end{tabular}


To further illustrate the rapid speed of ELM, Table 5 shows the comparisons of the training times as well as the testing times that are used for the SLFNs trained with the BP, the RBF, the SVM, the random weight feed-forward network and the ELM, respectively. Obviously, the ELM out-performs the others and the time is larger when more hidden nodes are applied.

Table 5. Computation of training times

\begin{tabular}{|c|c|c|}
\hline Algorithm & Training Time(s) & Testing Time(s) \\
\hline BP & 15.7341 & 0.0928 \\
\hline SVM & 18.8926 & 0.1753 \\
\hline RBF & 0.1096 & 0.0562 \\
\hline Feed-Forward(50 hidden nodes) & 0.0981 & 0.0541 \\
\hline ELM(50 hidden nodes) & 0.0977 & 0.0662 \\
\hline ELM(100 hidden nodes) & 0.1218 & 0.0830 \\
\hline ELM(300 hidden nodes) & 0.3237 & \\
\hline
\end{tabular}

\subsection{Summary}

In this chapter, the SLFN trained with the ELM has been used for modeling the thermodynamics behavior of proposed hybrid refrigeration cycle. It has been seen that the designed SLFN model trained with the ELM achieve the most satisfying performance with smallest error as well as highest robustness compared with other intelligent methods such as BP, RBF and SVM. The testing results have further revealed the excellent prediction accuracy, efficiency and robustness of the SLFNs trained with the ELM. In the next chapter, the model-based optimization with ELM training for proposed hybrid cycle will be presented. 


\section{Chapter 6 Optimization of Hybrid Ejector-based Air- conditioning System with ELM}

In the previous chapter, the hybrid system is modeled with different types of ANN, and the comparison result indicates that ELM out-perform the others. Therefore, in this chapter, the optimization of hybrid system with ELM is further investigated.

\subsection{Problem formulation}

The goal towards hybrid ejector refrigeration system optimization issue is to achieve minimum energy consumption while satisfying the requirement of cooling capacity at the same time. Thus, according to the result provided by boosting tree algorithm in Table 3 and the input selection in (92), these six inputs are capable of predicting the system total energy consumption. Then, to transfer the problem into mathematical expression, the optimization issue can be built up as

$$
\begin{aligned}
& \text { Min } \dot{W}_{\text {total }}=f\left(f_{\text {com }}, f_{\text {con }}, f_{\text {eva }}, A_{v}, P_{c o n, i n}, P_{e, p, i n}\right) \\
& \text { Subject to } \dot{Q}_{e}=\dot{Q}_{d e}
\end{aligned}
$$

where $W_{\text {total }}$ and $Q_{d e}$ are total electrical energy consumption of the system and cooling capacity in demand, respectively.

With problem formulated, the next step is to ascertain the feasible operating range of the system, so that the solution obtained later is practical guaranteed. And based on experimental results, the corresponding constraints are listed as following:

1. Operation frequency of condenser and evaporator fans

$$
\begin{aligned}
& f_{\text {con,min }} \leq f_{\text {con }} \leq f_{\text {con,max }} \\
& f_{\text {eva,min }} \leq f_{\text {eva }} \leq f_{\text {eva,max }}
\end{aligned}
$$

where $f_{c o n, \min }$ and $f_{\text {con, } \max }$ are minimum and maximum condenser fan frequency, while $f_{\text {eva,min }}$ and $f_{\text {eva,max }}$ are minimum and maximum evaporator fan frequency, respectively.

2. Inlet and outlet temperature of condenser and evaporator 


$$
\begin{gathered}
T_{\text {outdoor }} \leq T_{\text {con,out }} \leq T_{\text {con, } \text { min }} \leq T_{\text {con,sat }} \leq T_{\text {con, } \max } \leq T_{\text {con, in }} \\
T_{\text {eva,in }} \leq T_{\text {eva,min }} \leq T_{\text {eva,sat }} \leq T_{\text {eva,max }} \leq T_{\text {indoor }}
\end{gathered}
$$

where $T_{c o n, \min }, T_{c o n, \max }, T_{c o n, s a t}$ are minimum, maximum and saturation condensing temperatures, while $T_{\text {eva,min, }} T_{\text {eva,max }}, T_{\text {eva,sat }}$ are minimum, maximum and saturation evaporating temperatures, respectively.

3. Condensing pressure and evaporating pressure

$$
\begin{gathered}
P_{c o n, \min } \leq P_{c o n} \leq P_{c o n, \max } \leq P_{\text {critical }} \\
P_{\text {eva, } \min } \leq P_{\text {eva }} \leq P_{\text {eva, } \max }
\end{gathered}
$$

where $P_{c o n, \min }, P_{c o n, \max }, P_{\text {eva,min }}, P_{\text {eva,max }}$ and $P_{\text {critical }}$ are minimum and maximum condensing pressures, minimum and maximum evaporating pressures, ejector critical back pressure, respectively.

4. Ejector back pressure

$$
P_{e, \text { back }} \leq P_{\text {critical }}
$$

where $P_{e, b a c k}$ is the ejector back pressure, back pressure too high will cause the decrease of entrainment ratio, if back pressure surpass the back flow point, it will lead to even worse circumstance called back flow which can cause damage to not only ejector itself but the whole system instead. The entrainment ratio will decrease sharply within the subcritical region. The critical pressure value and back flow point depend on the ejector geometrical configurations and is shown in Figure 83. 


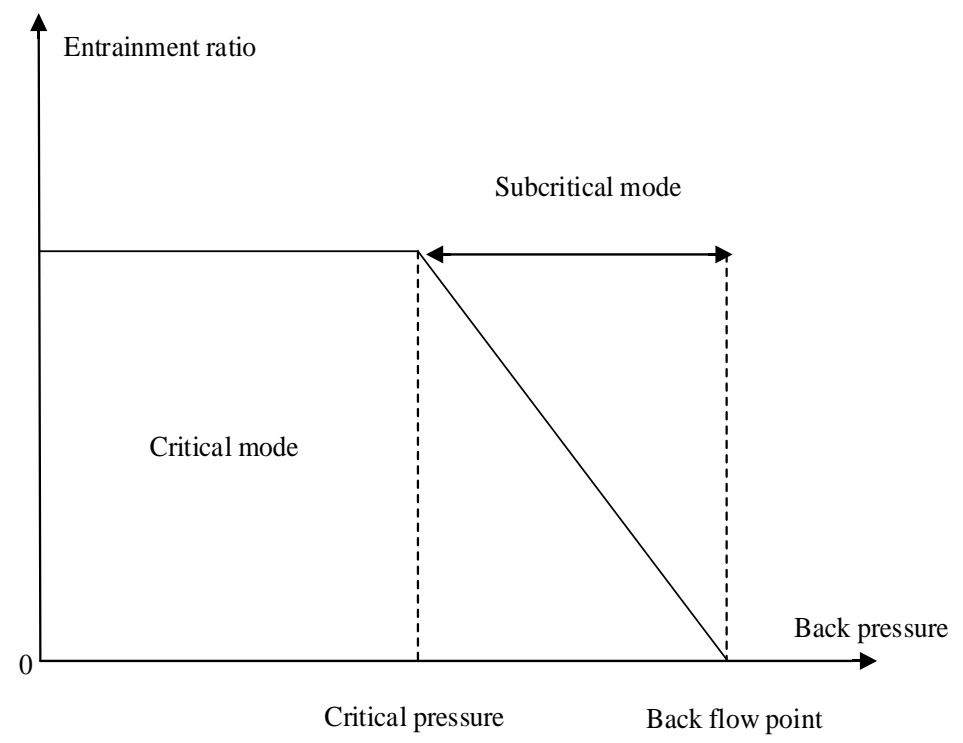

Figure 83 Ejector modes description

5. Liquid pump flow rates

$$
\dot{m}_{p u m p, \min } \leq \dot{m}_{\text {pump }} \leq \dot{m}_{p u m p, \max }
$$

where $\dot{m}_{\text {pump,min }}$ and $\dot{m}_{\text {pump,max }}$ are the minimum and maximum flow rates generated by liquid pump.

6. Compressor Frequency

$$
30 \mathrm{~Hz} \leq f_{\text {com }} \leq 50 \mathrm{~Hz}
$$

The upper and lower bounds of compressor frequency are in accordance with applied compressor working range provided by manufacture.

7. Refrigerant mass flow rate

$$
\dot{m}_{r, \min } \leq \dot{m}_{r}=\dot{m}_{s}+\dot{m}_{p} \leq \dot{m}_{r, \max }
$$

where $\dot{m}_{p}$ and $\dot{m}_{s}$ are ejector primary and secondary mass flow rate, and $\dot{m}_{r, \min }$ and $\dot{m}_{r, \max }$ are minimum as well as maximum refrigerant mass flow rate, respectively. The higher extreme value about total refrigerant mass flow rate is depended on opening condition of electric expansion valve, liquid pump operation conditions such as frequency, and compressor capacities, while the minimum value is determined by accuracy and operating range of installed flow meters.

8. EEV opening percentage 


$$
30 \%<A_{v}<60 \%
$$

After the determination of physical constraints of relative components of proposed system, the optimization issue can be preceded based on following parts:

\section{Pre-data processing:}

Before the implementation of optimization algorithm, some preliminary treatments towards raw data collected directly from experimental platform need to be done. Generally, when dealing with the raw experimental data, the first thing is to carry out normalization procedure. Since the measured parameters are usually located within different ranges, and the influence of parameters can be largely affected by the magnitude difference, it is necessary to implement normalization towards the raw data to let them falling in a specifically given interval so that the influence of magnitude is negligible. Besides, the normalized data is helpful for weight learning procedure as well as sufficient neurons training since its activation values are usually within $[0,1]$. Last but not the least, after the output obtained, un-normalization needs to be executed in order to convert calculated outputs back into practical magnitude. Otherwise, the output value may find meaningless in real situation.

The mathematical expression of normalization method can be presented as:

$$
\begin{aligned}
& x_{n}=\left(x-x_{\min }\right) /\left(x_{\max }-x_{\min }\right) \\
& y_{n}=\left(y-y_{\text {min }}\right) /\left(y_{\text {max }}-y_{\text {min }}\right)
\end{aligned}
$$

where $x_{\max }, x_{\min }, y_{\max }$ and $y_{\min }$ are the maximum and minimum values of the input and output data series, respectively.

Then, with normalized input and output data, it is essential to evaluate the system operation state before carrying out system energy consumption model. For evaluating the state, it is meant to ascertain the system stability under certain operating point in this case. And it can be considered as a normal classification problem with training sets labeled to indicate the system states. Usually, the positive label means that the system is stable while the negative label indicates the instability of system. In order to achieve satisfying performance of classification, the parameters selected to represent the system states are very important. The selected features should satisfy the demand that different patterns can be separated as distinct as possible. The principal component analysis (PCA) is applied 
here to concentrate much of the signal into a few principal components and thus realize dimensionality reduction. The selected features are shown as follows to represent the states of hybrid system:

$$
x=\left[\begin{array}{llllll}
f_{\text {com }} & f_{\text {con }} & f_{\text {eva }} & A_{v} & T_{\text {amb }} & T_{\text {in }}
\end{array}\right]
$$

The features selected together with cooling capacity constraint are sufficient for system stability distinguish. Thus the $Q_{e}$ is added to form the final input for classifier. Since each feature has its own physical constraint, and all these constrained operating ranges combine to form into a high dimensional permissible operating region. Within this region, the classification is implemented. Additional, the ANN built for classification can be illustrated in Figure 84

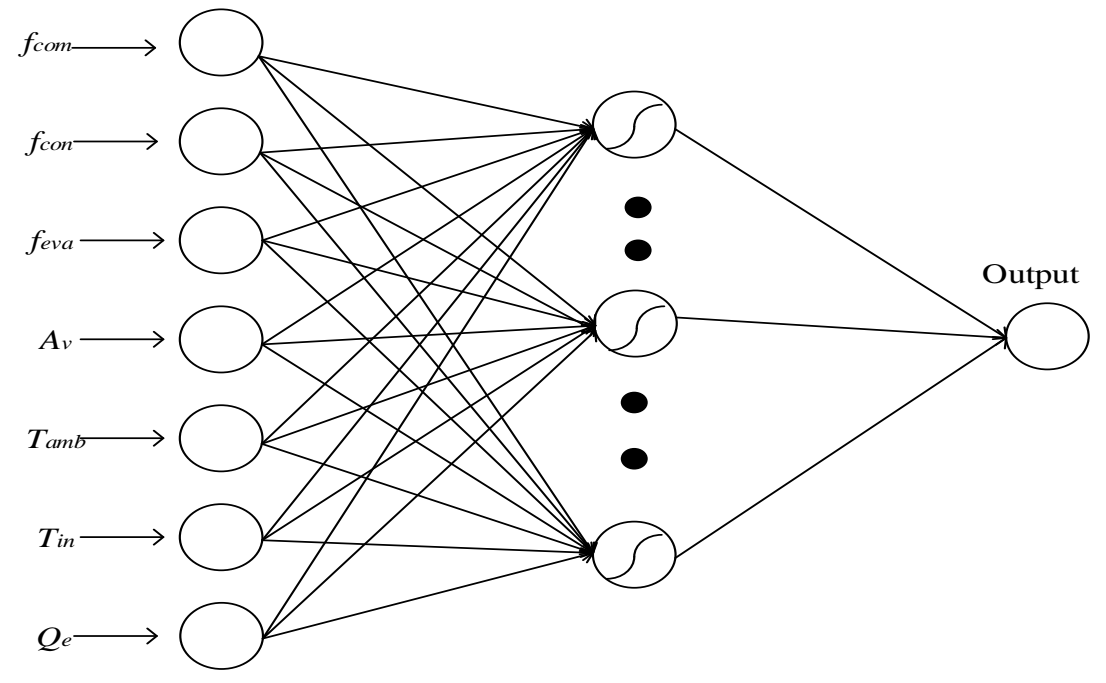

Figure 84 ANN for system stability classification

The most commonly used activation function, sigmoid function, is applied in the hidden layer. The mathematical expression is shown below. And the performance of training and training efficiency are considered to help determine the appropriate number of hidden nodes.

$$
f(t)=\frac{1}{1+e^{t}}
$$

\section{Model built up:}

The most crucial ingredient of a successful optimization strategy in hybrid system is to acquire an accurate energy consumption model. For the proposed system, the total energy 
consumption consists of four variables, energy consumed by compressor, liquid pump, condenser fan and evaporator fan. Generally speaking, the operating frequencies of compressor, liquid pump, and fans of evaporator and condenser are highly related to their energy consumption value. Thus, these four parameters are applied for energy performance as well as indoor and ambient temperature and equality constraint cooling capacity demand. Therefore, there are total seven input nodes in the network. ANN with single hidden layer is also used for energy consumption model. The sigmoid function is used as transfer function in hidden layer. In order to easily evaluate prediction result of each component, one summation layer is added at the end with each weight equals to 1 . The weight between input layer and hidden layer is randomly assigned and the weight between hidden layer and output layer are determined based on manner of ELM. The ANN structure of system total energy consumption model is shown in Figure 85.

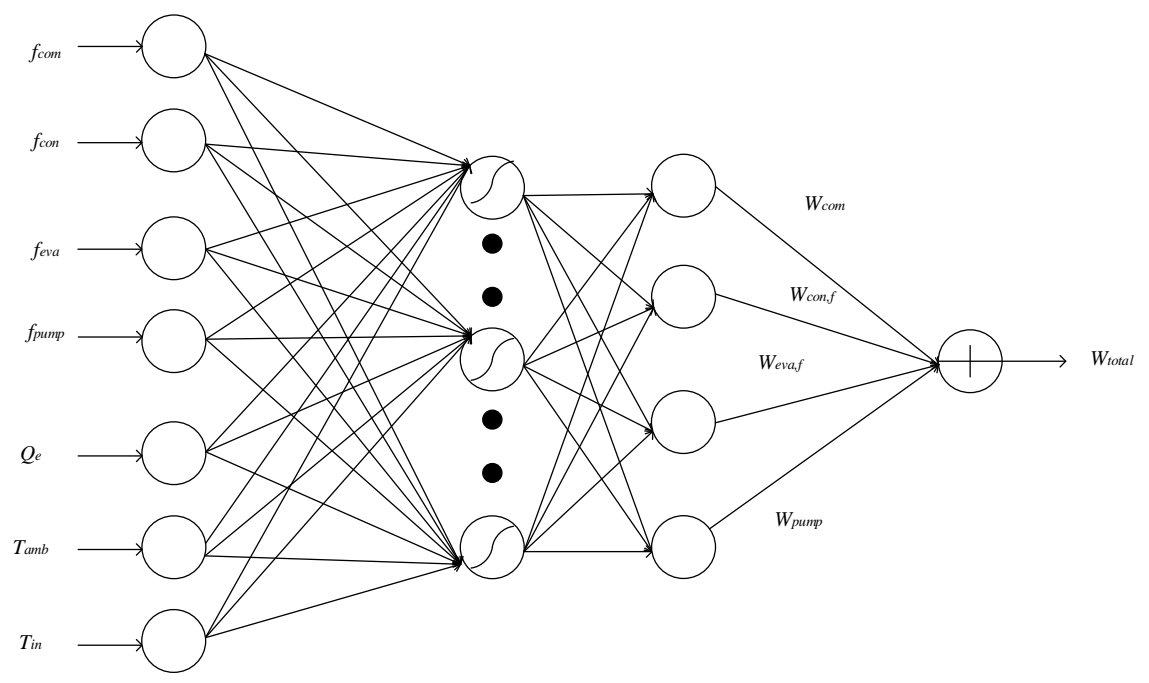

Figure 85 ANN structure of total energy consumption model

\section{Optimum solution search manner:}

Since the optimization of hybrid system possesses the character such as massive quantity of experiment data and nonlinearity, it is necessary to apply specific search method that can accurately locate the optimal solution without any missing information. The exhaustive grid search method is a suitable candidate. The idea of it is to search the whole assigned region exhaustively from lower bound to upper bound following the step size defined by user. Once the energy consumption is calculated, it will be stored and 
compared with new-coming result, the smaller one will be kept and waited to be compared with latter. This process continues until the whole region is searched. The pseudo code is illustrated as:

$$
\begin{aligned}
& \text { Initialization: Generate solution } Q_{e}, T_{a m b}, T_{i n} \text { and } W_{\text {total,min }} \\
& \text { for } f_{\text {com }}=f_{\text {com,min }}: f_{\text {com, step }}: f_{\text {com, } \max } \\
& \text { for } f_{\text {con }}=f_{\text {con,min }}: f_{\text {con,step }}: f_{\text {con, } \max } \\
& \text { for } f_{\text {eva }}=f_{\text {eva,min }}: f_{\text {eva,step }}: f_{\text {eva,max }} \\
& \text { for } f_{\text {pump }}=f_{\text {pump,min }}: f_{\text {pump,step }}: f_{\text {pump, } \max }
\end{aligned}
$$

Feasible check: $F=$ Function $\left(f_{\text {com }}, f_{\text {con, }}, f_{\text {eva }}, Q_{e}, T_{a m b}, T_{i n}\right)$

if $F$ is stable

Execute energy consumption model: $W_{\text {total }}=$ Function $\left(f_{\text {com }}, f_{\text {con }}, f_{\text {eva }}, f_{\text {pump }}, Q_{e}, T_{\text {amb }}, T_{\text {in }}\right)$

$$
\begin{gathered}
\text { if } W_{\text {total,min }}>W_{\text {total }} \\
W_{\text {total,min }}=W_{\text {total }}
\end{gathered}
$$

End

End

$$
\text { End }
$$

End

End

\section{End}

\subsection{Work flow of optimization}

\section{Step 1: Data acquisition}

Collect the data of selected parameter either through sensors or other equipment. Since the variables such as indoor and ambient temperature and demand cooling capacity are uncontrollable, it is practicable to consider their values as constants during the whole sampling period.

Step 2: Data normalization

Based on the equation mentioned before, all the data are normalized within $[0,1]$ for energy consumption model. 
Step 3: Input variables search

According to the exhaustive grid search method, the input variables are choice independently within each constrained range.

Step 4: Feasibility check

If the feasibility of current input is negative, then return to previous step, otherwise, continue.

Step 5: Execute energy consumption model

Assign the values obtained in step 3 to ANN input layer and obtain the total energy consumption.

Step 6: Comparison and storage

The first calculated total energy consumption is regarded as initialized $W_{\text {total,min }}$, and the latter obtained one is used to compare with the stored one, the smaller one will be stored and regarded as new optimal solution, then return to step 3 for next input variable.

Step 7: Termination

The process won't stop until all the selected variables reach their upper bound.

Step 8: Test and evaluation

The optimal solution can be tested and evaluated on the proposed experiment platform.

The detailed flowchart of optimization algorithm is described in Figure 86. 


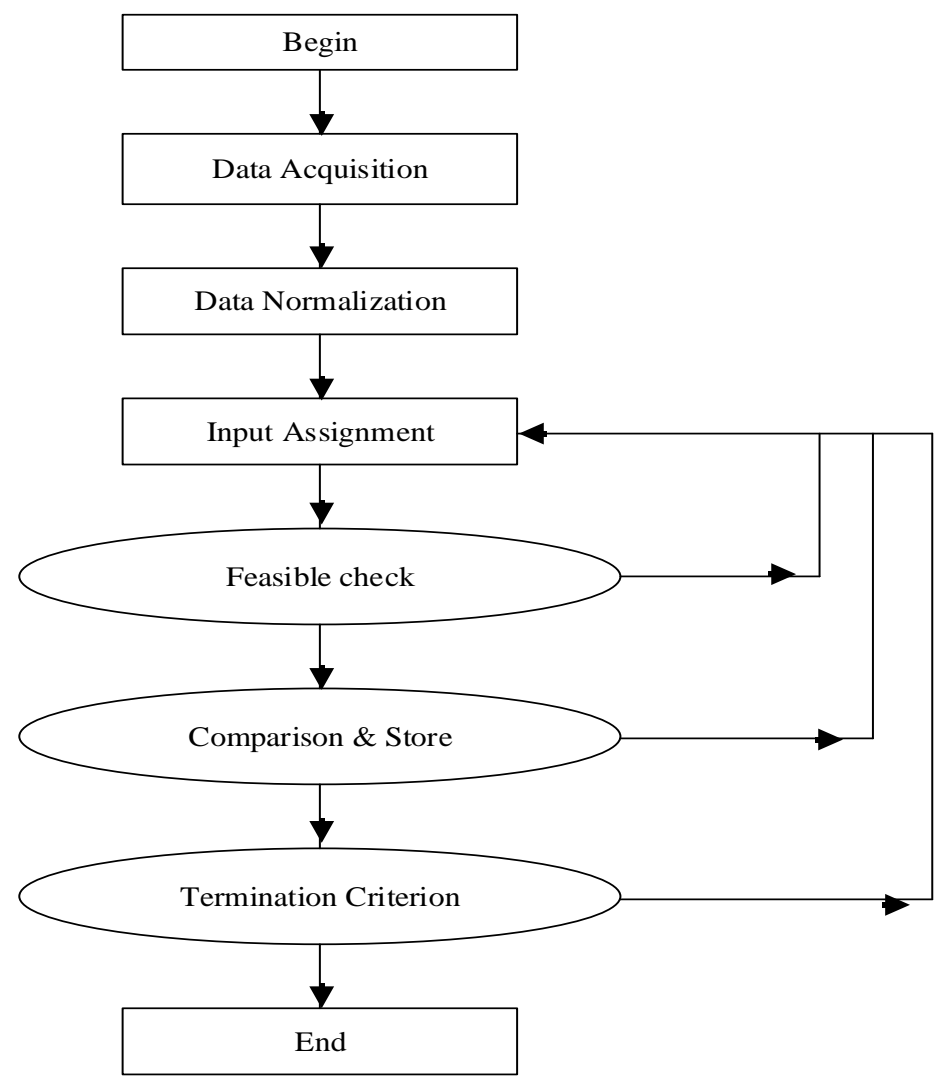

Figure 86 Flow chart of optimization algorithm

\subsection{Experiment results and discussion}

\subsubsection{Classification results and discussion}

The classifier is trained first by sample data obtained under different operating conditions within the permissible range. In order to select the method with best performance, the BP, SVM and ELM are implemented during the training. Then, 100 pairs of testing data are applied to evaluate the generalization performance of trained classifier.

The training and testing results of classifier are shown in Figures 87 and 88. 


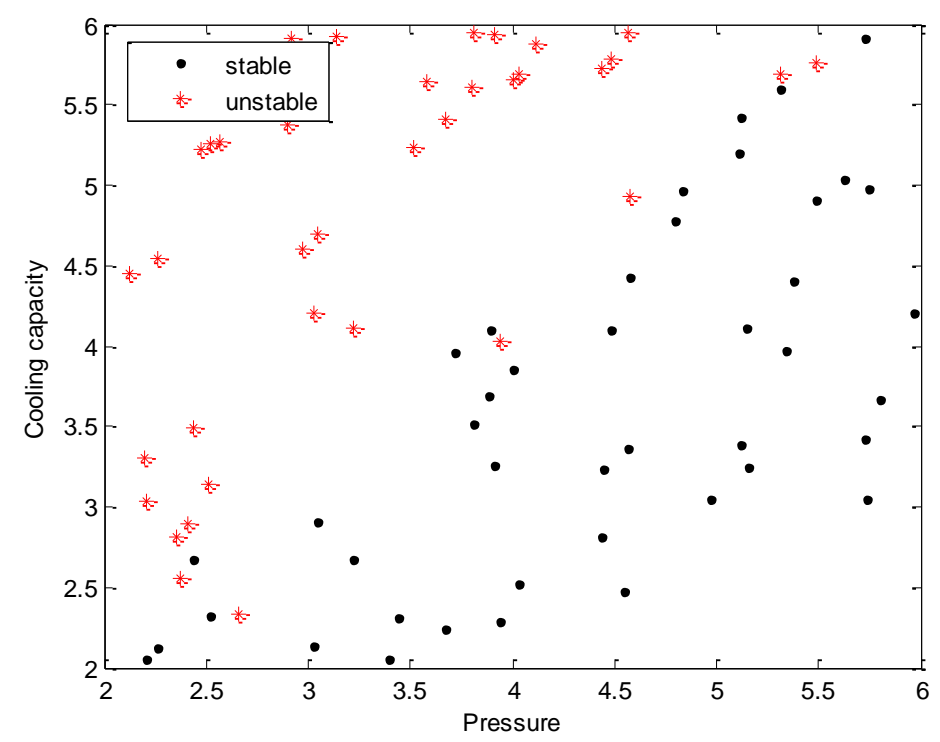

Figure 87 Training results of classification

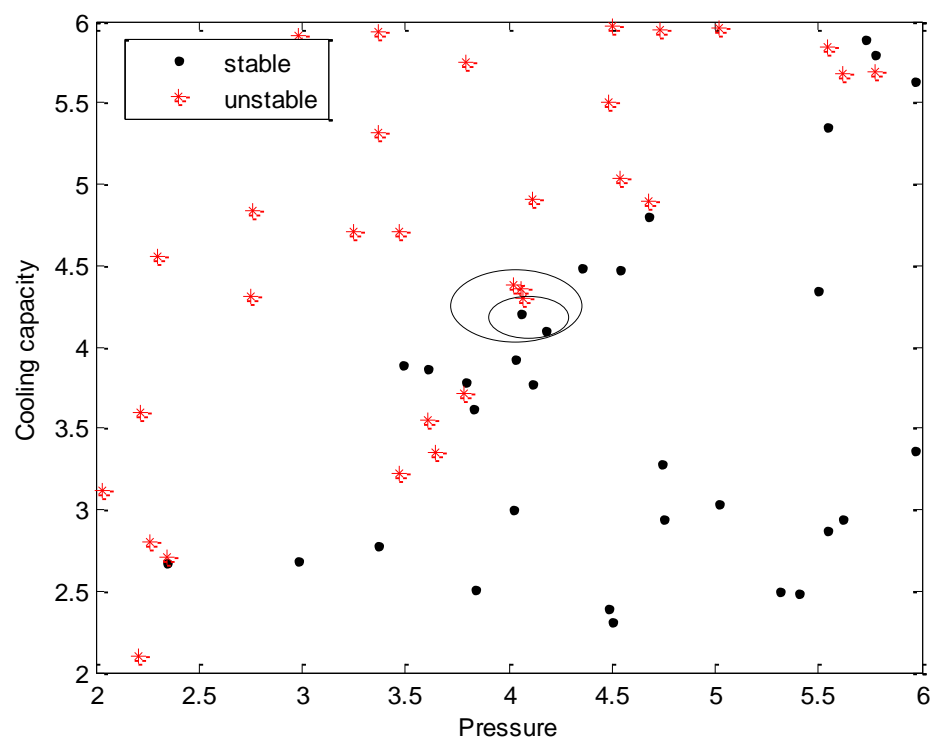

Figure 88 Testing results of classification

As evaporating pressure and cooling capacity are two important elements to help analyze whether system is stable or not, they will be applied and served as $\mathrm{x}$ and $\mathrm{y}$ axis of classification coordinate. Figures 87 and 88 show the training and testing results of classification problem with the BP, SVM and ELM, respectively. From the training result, it can be seen that no misclassification happens for all three employed algorithms. Nevertheless, there are several misclassifications occurring with the classifier trained with 
BP and SVM during testing part. To be specific, BP regards three unstable points as stable and two stable points as unstable, while SVM regards two stable points as unstable, respectively. The system may suffer from fluctuation caused by these misclassifications and may even cause severe breakdown. On the contrary, the performance of classifier trained with ELM is better than others for there is no misclassification towards all testing points. Besides, the high speed character of ELM makes it more competitive over the others. In this case, the time spent for training is $2.01 \mathrm{~s}$ for BP, 2.37s for SVM and $0.08 \mathrm{~s}$ for ELM, respectively. The efficiency advantage will be more noticeable with larger size of training sample. Therefore, ELM has shown its promising potential in on-line optimization application.

\subsubsection{Total energy consumption model results and discussion}

As mentioned before, ANN with single hidden layer trained by ELM is employed for total energy consumption model. In order to illustrate the performance of ELM, the network is also trained by BP and SVM for comparison purpose. The proposed network consists of seven input nodes, 30 hidden nodes and one output node. The training sample pairs are measured and collected under different cooling capacity. The corresponding output values are obtained via power meter. The fitting results of four energy consumed components are shown in Figures 89-96. 


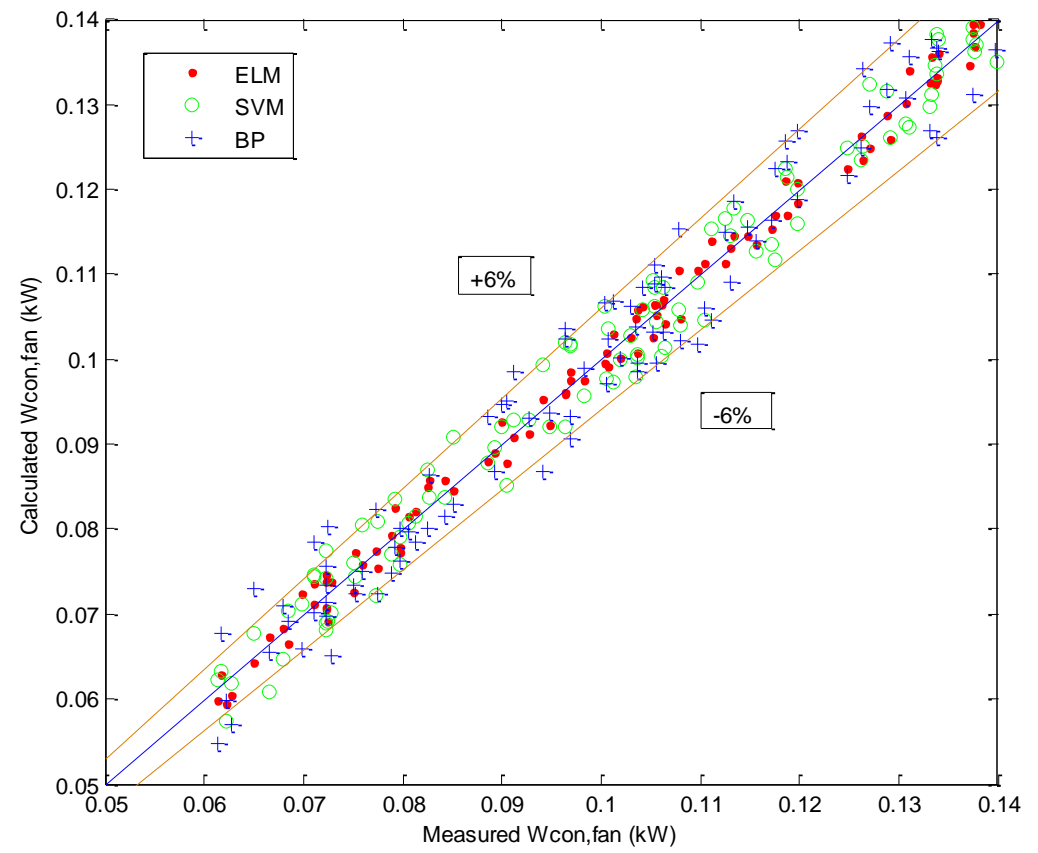

Figure 89 Fitting result of condenser fan energy consumption

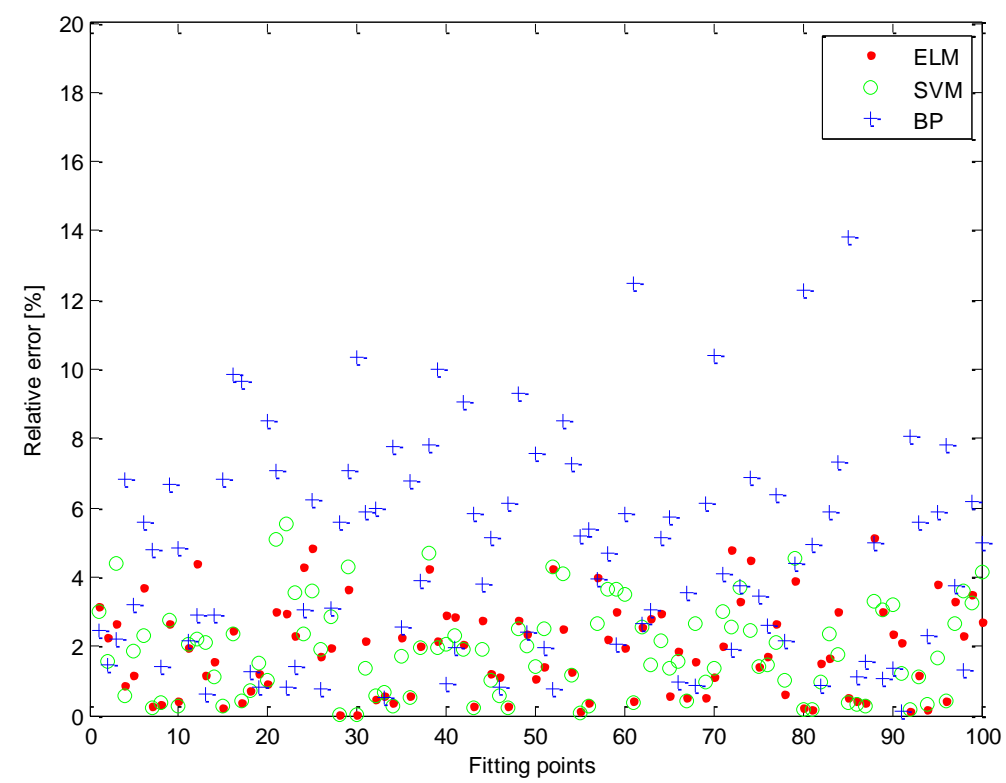

Figure 90 Relative errors of condenser fan model 


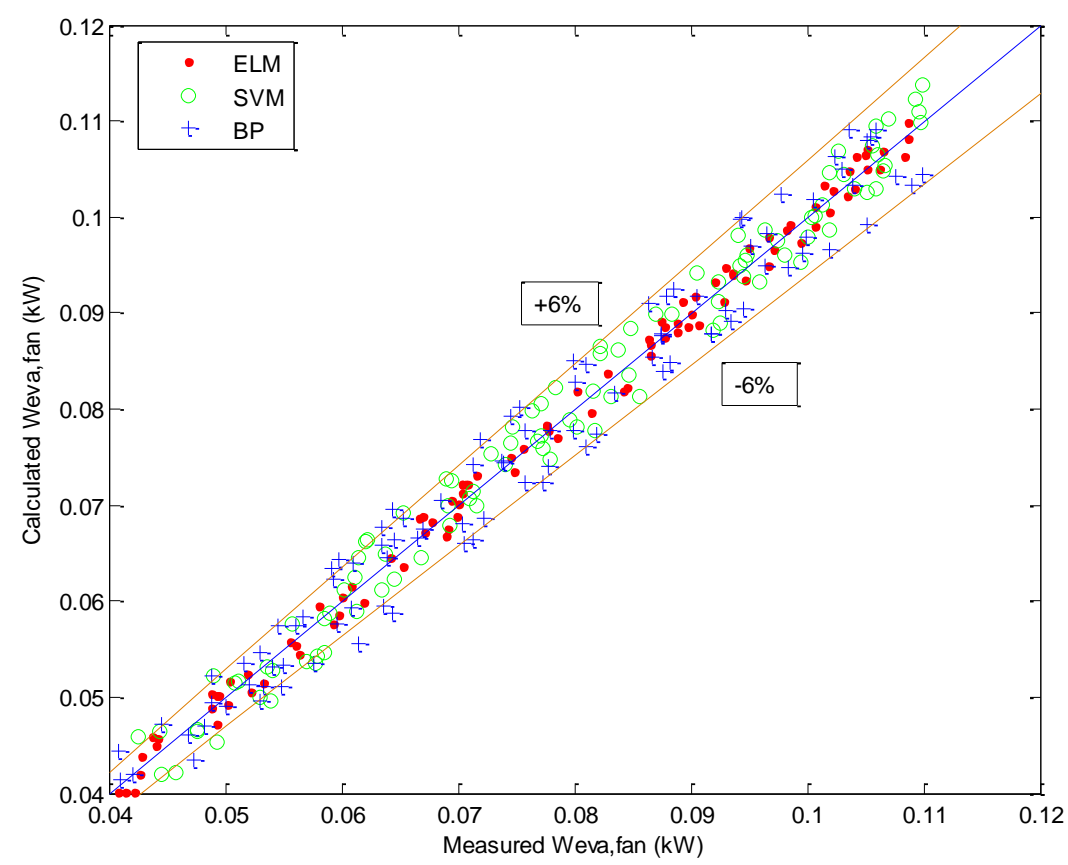

Figure 91 Fitting result of evaporator fan energy consumption

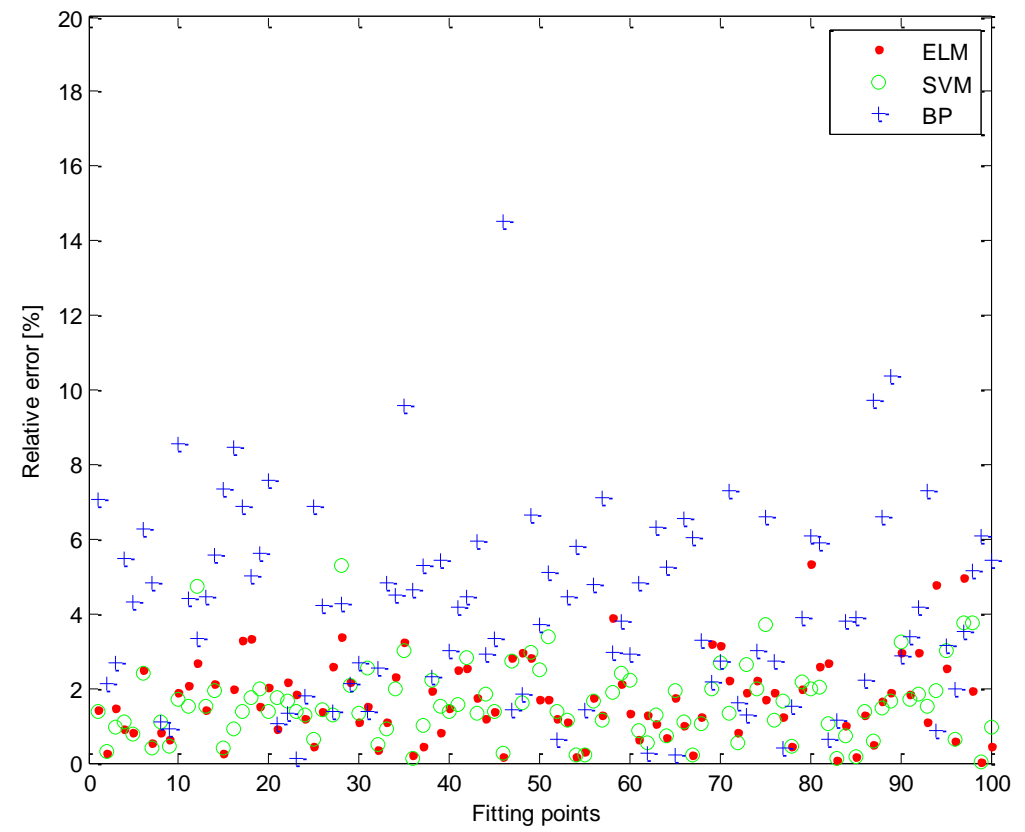

Figure 92 Relative errors of evaporator fan model 


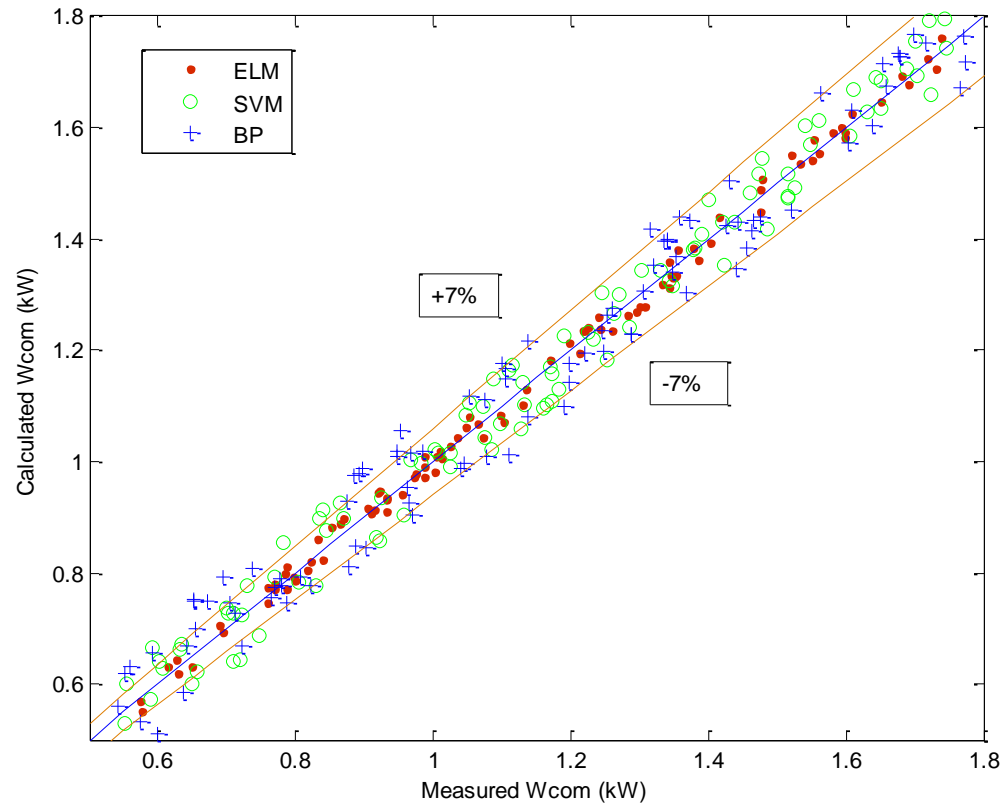

Figure 93 Fitting result of compressor energy consumption

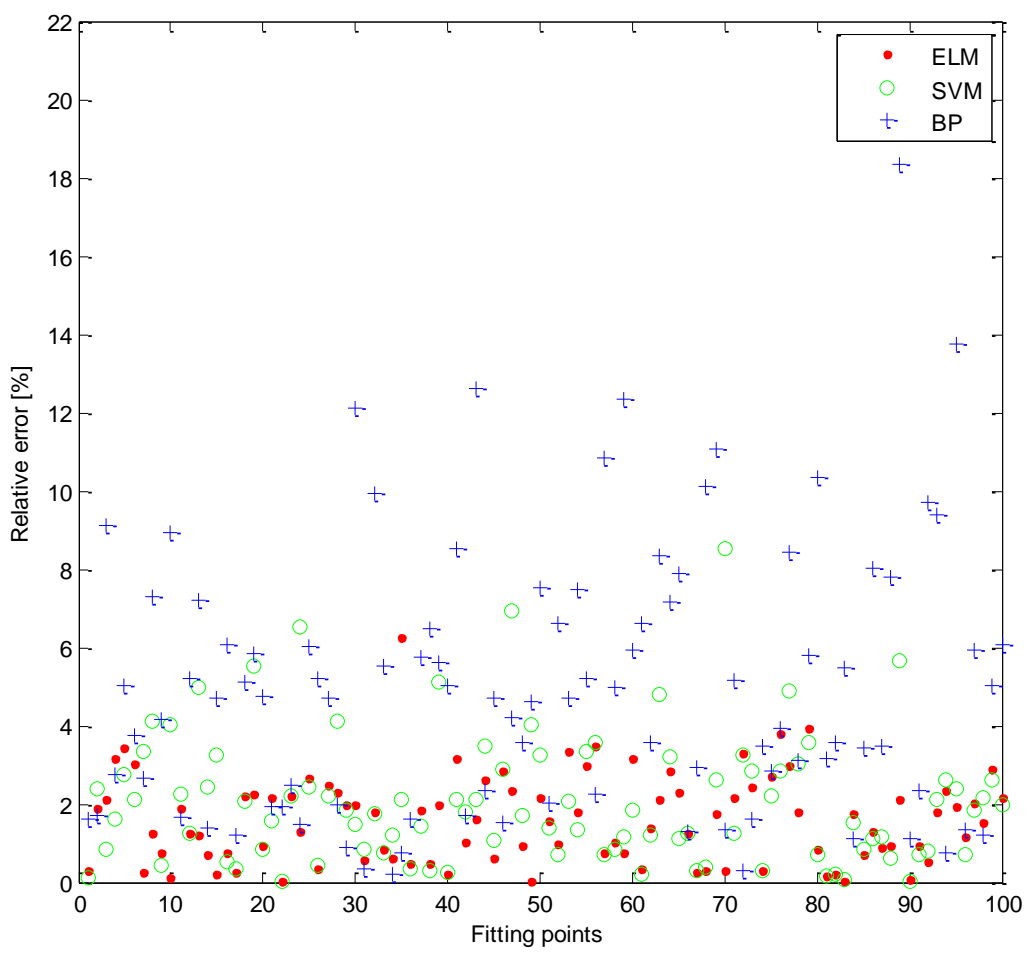

Figure 94 Relative errors of compressor model 


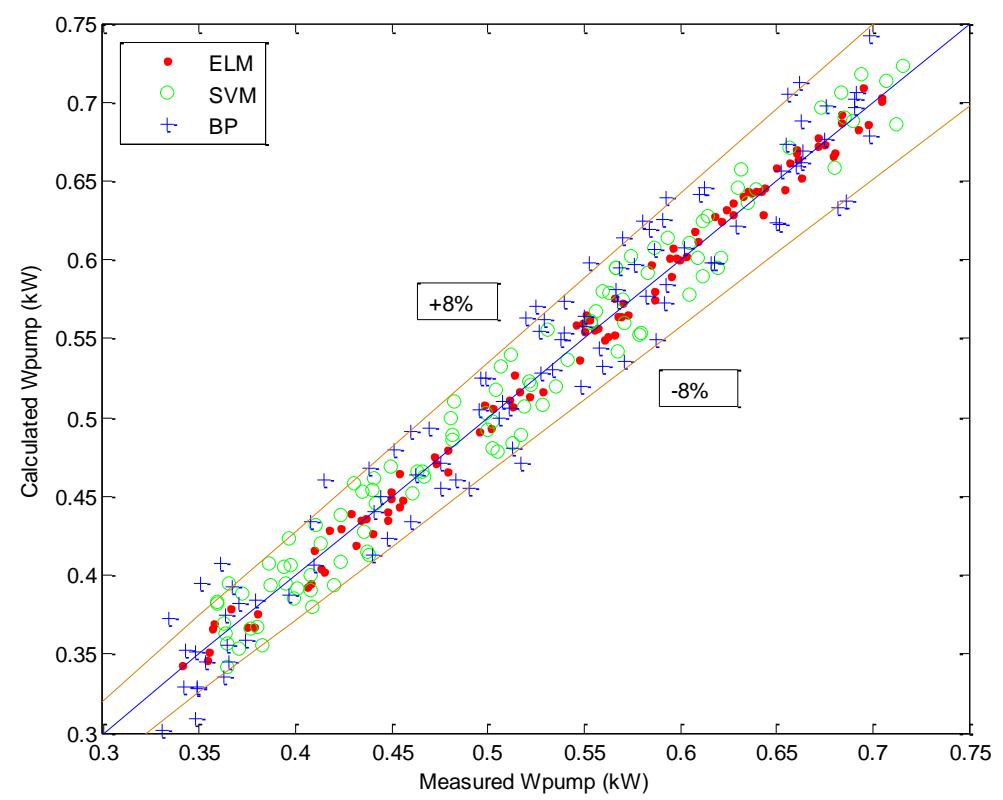

Figure 95 Fitting result of liquid pump energy consumption

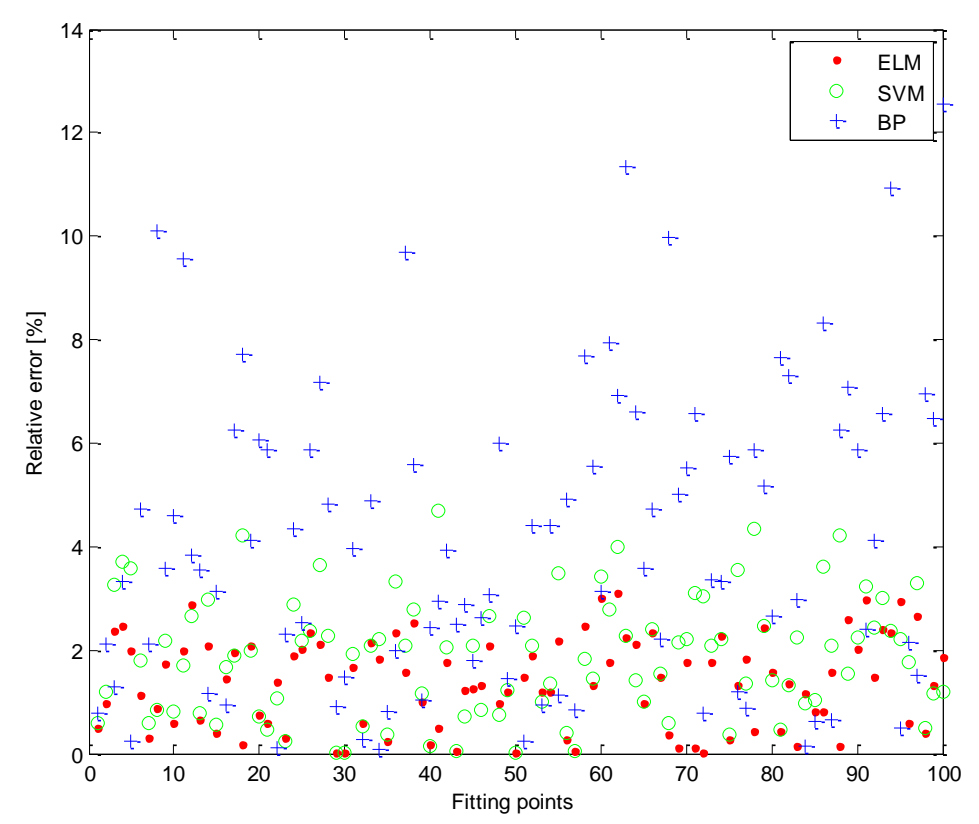

Figure 96 Relative errors of liquid pump model

From Figures 90, 92, 94 and 96, respectively, it can be found that the fitting results of ELM out-perform the others which affirm the previous speculation. To be more detailed, the majority of testing value of ELM has relative error within $\pm 5 \%, \pm 4 \%, \pm 4 \%$ and $\pm 3 \%$ for energy consumption of condenser fan, evaporator fan, compressor and liquid pump. 
While the deviation for BP and SVM are $\pm 10 \%, \pm 9 \%, \pm 12 \%, \pm 8 \%$ and $\pm 6 \%, \pm 5 \%, \pm 8 \%$, $\pm 5 \%$, respectively.

With classifier and energy consumption model validated, the proposed optimization algorithm was carried out. The system was operated for 24 hours and the data was collected per hour in order to calculate the total energy consumption variation. The most commonly used on-off control is applied for comparison and the result is shown in Figure 97.

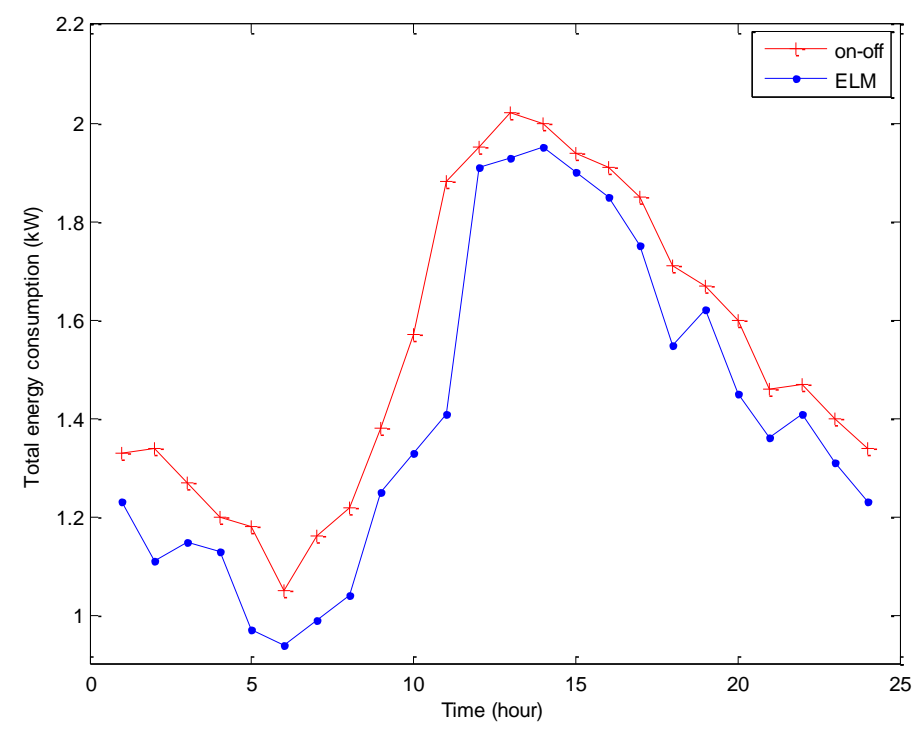

Figure 97 Comparison results of proposed optimization algorithm

Taken a simple point for example, the environment data was collected first; then followed by data pre-processing, such as normalization and input determination; after this, the feasible check was conducted, only the feasible variables were passed on to obtain the related energy consumption; later, the comparison between obtained energy consumption value was made and the smaller one was stored; this process was iterated until terminate criterion was reached. Therefore, the final obtained values were regarded as the optimal value at that point. From the comparison result, it can be implied that the ELM based optimization algorithm is more energy efficiency than traditional on-off control. Additional, the energy saving benefit is lower when the cooling capacity is around its peak value. The optimal saving effect happens with the cooling capacity is about $65-85 \%$ of the full load. The average energy conservation value using proposed algorithm is $5.83 \%$ 
lesser than that of on-off control which is calculated by $\frac{1}{n} \sum_{1}^{n} \frac{\left|W_{E L M, n}-W_{\text {on-off }, n}\right|}{W_{\text {on-off }, n}} \times 100 \% \quad n=1,2, \ldots 24$.

\subsection{Summary}

In this chapter, an optimization approach towards proposed hybrid system based on ANN modeling is presented. The stability of system is ascertained through the proposed classifier and then the system total energy consumption is obtained via ANN trained with ELM. From the training and testing results, it can be found that classifier trained with ELM has $100 \%$ accuracy during training and testing and exhibits better generalization performance than the one with BP and SVM. Besides, the rapid speed of ELM makes it more appealing and competitive. For the result of energy consumption models, ELM also out-performs the other methods with smallest relative error during both training and testing. The proposed ELM based optimization algorithm is applied on the hybrid system that is operated for a whole day. The comparison result between ELM and traditional onoff control indicates that proposed algorithm is more energy saving. Additional, the energy saving effect will become more notable when the cooling capacity is around 65-85\% of its peak value. Moreover, the overall energy consumption with proposed algorithm employed is $5.83 \%$ less than that with on-off for one typical day. The simulation and experiment results show that the optimization algorithm based on ELM can effectively optimize system operation. 


\section{Chapter 7 Optimization of Hybrid System with PSOGA}

Although the performance of ELM optimization approach was satisfying, the exhaustive grid search method may not seem to be efficient or intelligent. It is of interests to see whether there exist some aspects to be improved. Therefore, the bionics optimization algorithms which are inspired by behavior of living things came into our sight. In this chapter, the performance of a newly developed PSOGA optimization algorithm was tested with discussion.

\subsection{Introduction}

In order to achieve more energy saving effect on hybrid ejector-based refrigeration cycles, it is necessary and helpful to review the research works about how to improve the system's coefficient of performance (COP). [111] presented a new method of thermal and economical optimum design of vapor compression refrigeration system. The objective function for optimization was the total cost per unit cooling load of the system. In order to obtain the optimal solution, the Lagrange multiplier method was applied on objective function. The simulation and experiment was done under different operation conditions to evaluate the performance and sensitivity of proposed method, and the results indicate a satisfying improvement. [112] introduced the finite time thermodynamic models to vapor compression system and other three modified vapor compression systems with respect to the heat exchanger parameters. The models were used for investigating the optimum performance with respect to inter-stage temperature and sub-cooler saturation temperature. Since the finite time thermodynamic models did not take the irreversible losses due to non-isentropic compression or expansion processes into account, the actual calculated COP value might be less than the one presented. [113] also applied finite time thermodynamic approach to obtain the optimum performance of an irreversible heat engine-driven combined vapor compression and absorption refrigerator system. The effect of thermal resistances and internal irreversibility on the performance of combined cycle was considered in order to be more practical. [114] proposed an optimal control strategy to help determine the maximum COP of a multi-evaporator vapor compression system with different cold storage rooms. The optimal control system was a MIMO and affine nonlinear model. The compressor speed and EEV opening percentage were varied to 
accommodate different cooling demands. The performance of proposed strategy was evaluated by simulation tests and the results implied that system energy consumption can be saved associated with this optimal method compared with fixed set-point control strategies. [115] investigated the effect of compressor discharge temperature set-points on optimization of energy efficiency of a variable speed vapor compression system. A model-free extreme seeking algorithm was applied due to its dramatically faster speed and more reliable convergence properties. The author showed that the relationship between compressor discharge temperature and power consumption is convex and time-varying extreme seeking was used to minimize power. The results were compared with traditional perturbation-based seeking algorithm and demonstrated that the COP of system using proposed algorithm achieve an increase of 14\%. [116] applied a lumped parameter moving boundary modeling to heat exchanger in vapor compression systems and then designed a model predictive controller to determine the optimal control actions based on dynamic exergy-based objective function. The simulation results revealed that proposed controller can achieve over $40 \%$ greater exergetic efficiency during operation. Moreover, the distribution of exergy destruction across individual components provided new insight into the effect of variable-speed actuators on vapor compression system efficiency.

Due to the fact that the components of hybrid ejector-based air conditioning system are highly coupled and mutual effected, the solution provided by conventional approaches is more likely to miss the global minimum but rather be trapped in local minimum. While, with the development of intelligent algorithm, more and more research have been done on application of this new techniques, which includes the optimization of HVAC system. [117] described the use of genetic algorithm (GA) for operating standard HVAC systems in order to achieve optimal performance, primarily with regard to power saving. Since the optimization problems of HVAC are nonlinear and discontinuous with complex limitations, GA was more applicable for its qualities of robustness and efficiency. A simulation was conducted to evaluate the performance of GA based $\mathrm{CO}_{2}$ concentration control in terms of energy saving amount and it was verified with energy software as well. The result indicated the expected saving effect was achieved. [118] proposed an adaptive learning algorithm based on GA for automatic tuning of PID controllers in HVAC system to achieve optimal performance. The modular dynamic simulation software package 
HVACSIM+ was modified and incorporated in GA based optimization program to provide a complete simulation environment for detailed study of controller performance. The system characteristics of overshoot, settling time and mean squared error were taken into consideration in the formulation of the objective function. The optimal controller parameters can be successfully determined by proposed program with $0.381^{\circ} \mathrm{C}$ overshoot, 14.5s settling time which yielded a better performance than that of traditional ZieglerNichols method. [119] introduced a model-based optimization strategy for condenser water loop of HVAC systems via separately dealing with analysis about characteristics of each component as well as processing the interactions within components. The objective of the built optimization problem was to minimize the total running price with regard to relative constraints. The optimal set points of the process were obtained via a modified GA under different cooling loads and varied ambient environment. Both simulations and experiments showed a great potential of proposed method in reducing energy consumption. [120] applied multi-island GA to decide the optimal running pattern of HVAC with minimum energy consumption. Dynamic heat load analysis for apartment house was made to analyze energy consumption and help select the potential configuration that could be energy saving. The results showed proposed method is significantly capable of determining optimal system design. [121] derived a unified method for efficient management of multiple chiller system by means of a particle swarm optimization (PSO) based algorithm. To achieve optimal performance in terms of reducing both power consumption and operative costs, as well as granting good load tracking properties, multi-objective functions were simultaneously solved. The advantages of PSO were that it requires a very limited setting of parameters and is easily extendable by inserting extra penalty terms. The results showed that PSO satisfactorily deals with such kind of nonlinear constrained optimization problem with ease of implementation. [122] presented a data-driven approach for minimization of HVAC energy consumption and room temperature ramp rate. Additional, computational intelligence algorithms were applied to solve the non-parametric model. Experiments were conducted to make a comparison between three intelligence algorithm, namely evolutionary algorithm (EA), harmony search (HS) and PSO. The PSO out-performed the other two in terms of its computational speed and quality of solutions. Moreover, three different simulation cases 
were analyzed based on proposed optimization model and the energy saving could be achieved by optimizing the settings for the supply air static pressure set point and discharged air temperature set point.

In this chapter, a PSOGA approach that combines PSO and GA is proposed to deal with the formulated centralized optimization problem of hybrid system. The object is to minimize the total energy consumption which is consisted of hybrid models of major energy consuming components. And the hybrid models of evaporator and condenser are applied to predict the thermodynamic performance of system. The derived PSOGA method is designed to seek the optimal solutions. Additional, experiments are conducted on hybrid system platform to evaluate the performance of presented method. From the results, it can be shown that a prominent reduction of energy consumption is achieved by proposed optimization strategy.

\subsection{Problem formulation}

\subsubsection{Objective function}

The objective of the optimization towards the hybrid system is described as ensuring the required cooling capacity while try to achieve this request with minimum energy spent at the same time. With the discussion before, it can be seen that the total energy consumption is made up of compressor, condenser fan, evaporator fan and liquid pump power consumption. Therefore, it can be mathematically built as

$$
\begin{aligned}
& \text { Min } \dot{W}_{\text {total }}=\dot{W}_{c o m}+\dot{W}_{c o n, f a n}+\dot{W}_{\text {eva }, f a n}+\dot{W}_{p u m p} \\
& \text { Subject to } \dot{Q}_{e}=\dot{Q}_{d e}
\end{aligned}
$$

where $W_{\text {com }}, W_{\text {con,fan }}, W_{\text {eva,fan }}$ and $W_{\text {pump }}$ are energy consumed by compressor, condenser fan, evaporator fan and liquid pump, respectively. The hybrid model of compressor will be adopted and the power consumptions of condenser fan, evaporator fan and liquid pump will be derived based on curve fitting of experiment data.

\subsubsection{Power consumption models of component:}

The compressor model developed before is the refrigerant energy change between compressor inlet and outlet, and the actual power consumption is expressed as 


$$
\dot{W}_{\text {com }}=\frac{\dot{W}_{\text {com, ideal }}}{\eta_{\text {com }}}
$$

where the $\eta_{\text {com }}$ is the efficiency coefficient of compressor and $W_{\text {com }}$ is the actual work done by compressor. And $\eta_{c o m}$ can be derived from

$$
\text { com }=a_{1}+a_{2}\left(P_{\text {com }, \text { out }} / P_{\text {com, in }}\right)+a_{3}\left(P_{\text {com, out }} / P_{\text {com }, \text { in }}\right)^{2}+a_{4}\left(P_{\text {com, out }} / P_{\text {com }, \text { in }}\right)^{3}
$$

where $a_{1}, a_{2}, a_{3}$ and $a_{4}$ are coefficient factors obtained via curved fitting.

The power consumption of liquid pump is affected by mass flow rate of refrigerant, pressure difference between pump inlet and outlet. And it can be mathematically expressed as

$$
\dot{W}_{\text {pump }}=\dot{m}_{p}\left(a_{1}+a_{2}\left(\frac{P_{\text {pump }, \text { out }}}{P_{\text {pump }, \text { in }}}\right)+a_{3}\left(\frac{P_{\text {pump }, \text { out }}}{P_{\text {pump }, \text { in }}}\right)^{2}+a_{4}\left(\frac{P_{\text {pump out }}}{P_{\text {pump }, \text { in }}}\right)^{3}\right)
$$

The power consumptions due to the operation of fans from both condenser and evaporator are determined by mass flow rates across fan and pressure difference between fan's inlet and outlet. Since the mass flow rate is proportional to operation frequency of fan, and the square of pressure is proportional to square of operation frequency of fan, the power consumption model of fans can be derived as:

$$
\begin{aligned}
& \dot{W}_{c o n, f a n}=a_{1}+a_{2} f_{c o n, f a n}^{\frac{1}{2}}+a_{3} f_{c o n, f a n}+a_{4} f_{c o n, f a n}^{2} \\
& \dot{W}_{e v a, f a n}=a_{1}+a_{2} f_{e v a, f a n}^{\frac{1}{2}}+a_{3} f_{e v a, f a n}+a_{4} f_{e v a, f a n}^{2}
\end{aligned}
$$

where $a_{1}, a_{2}, a_{3}$ and $a_{4}$ are parameters obtained through curved fitting based on experiment data.

The model of fans and liquid pump are evaluated based on data obtained from experiment, and the fitting results are shown in Figures 98-103, respectively. 


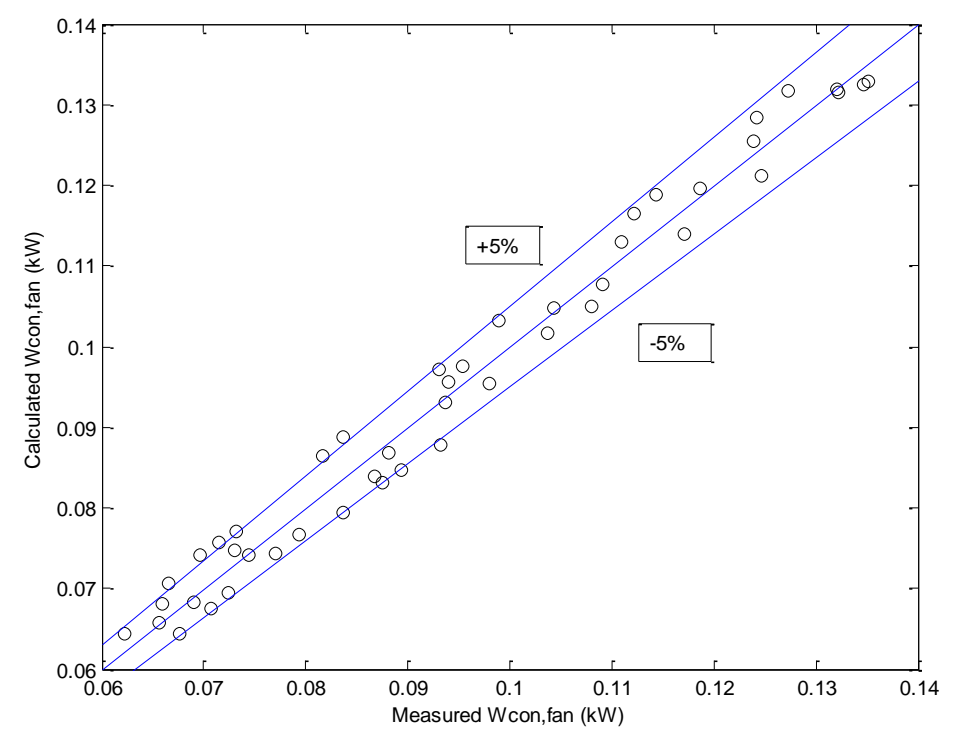

Figure 98 Fitting results of $W_{\text {con,fan }}$

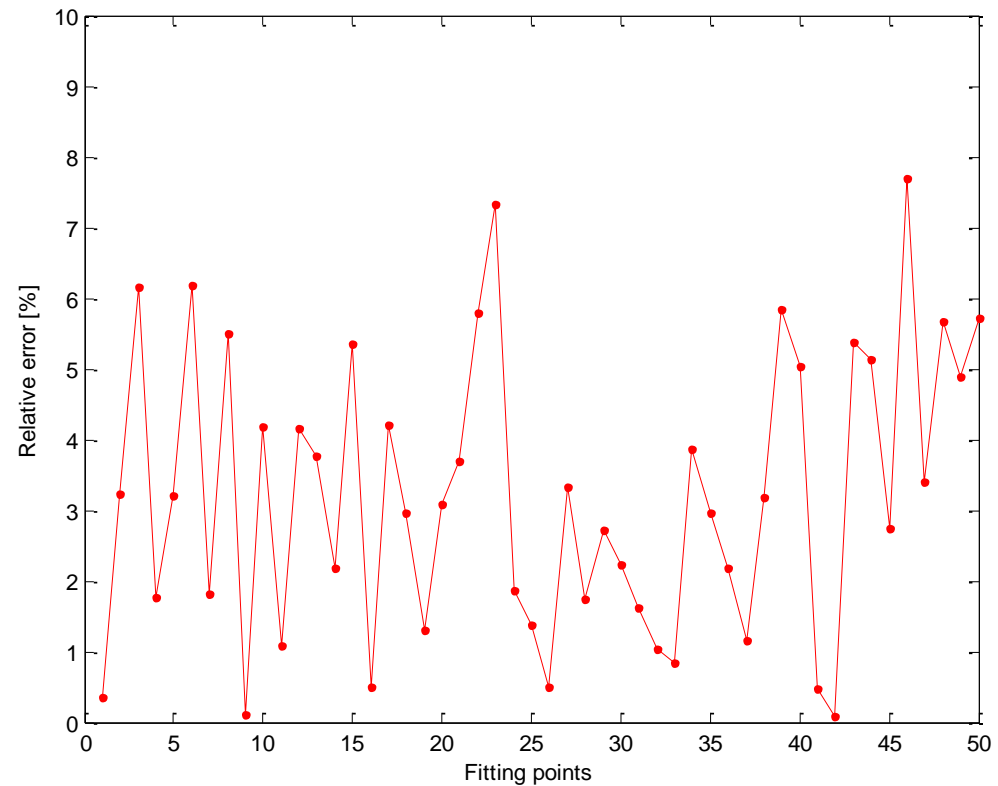

Figure 99 Relative error of $W_{c o n, f a n}$ 


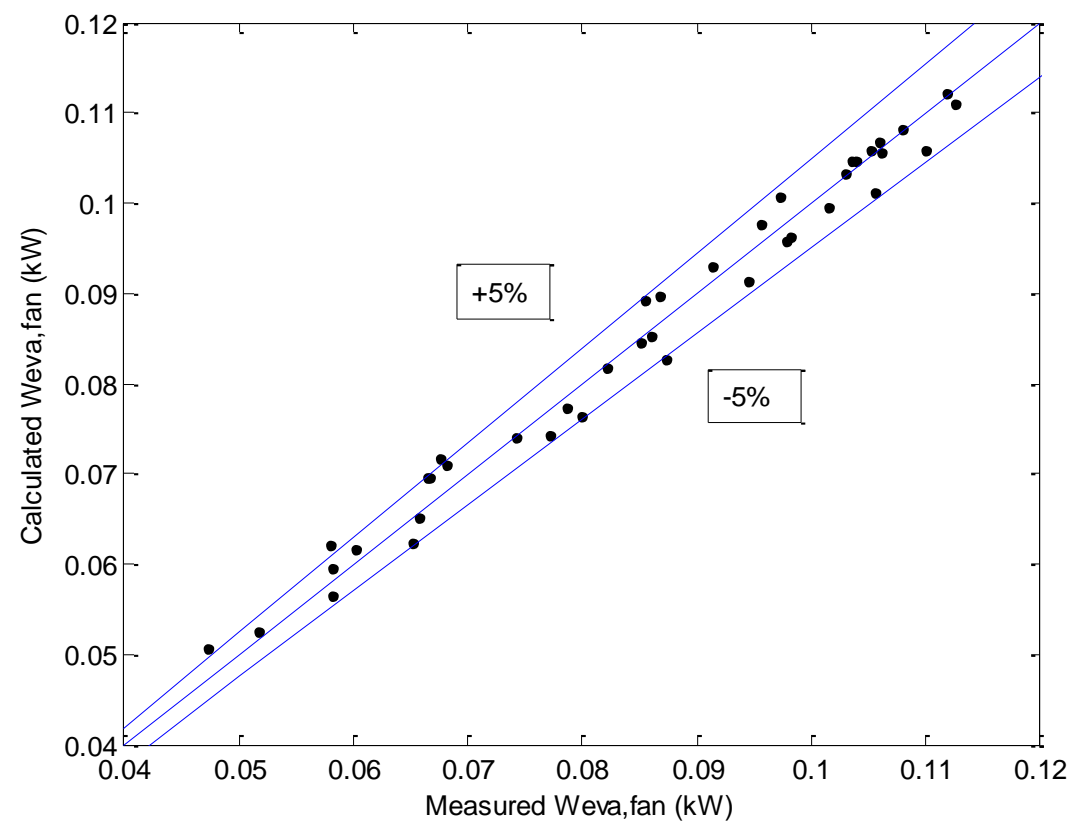

Figure 100 Fitting results of $W_{\text {eva,con }}$

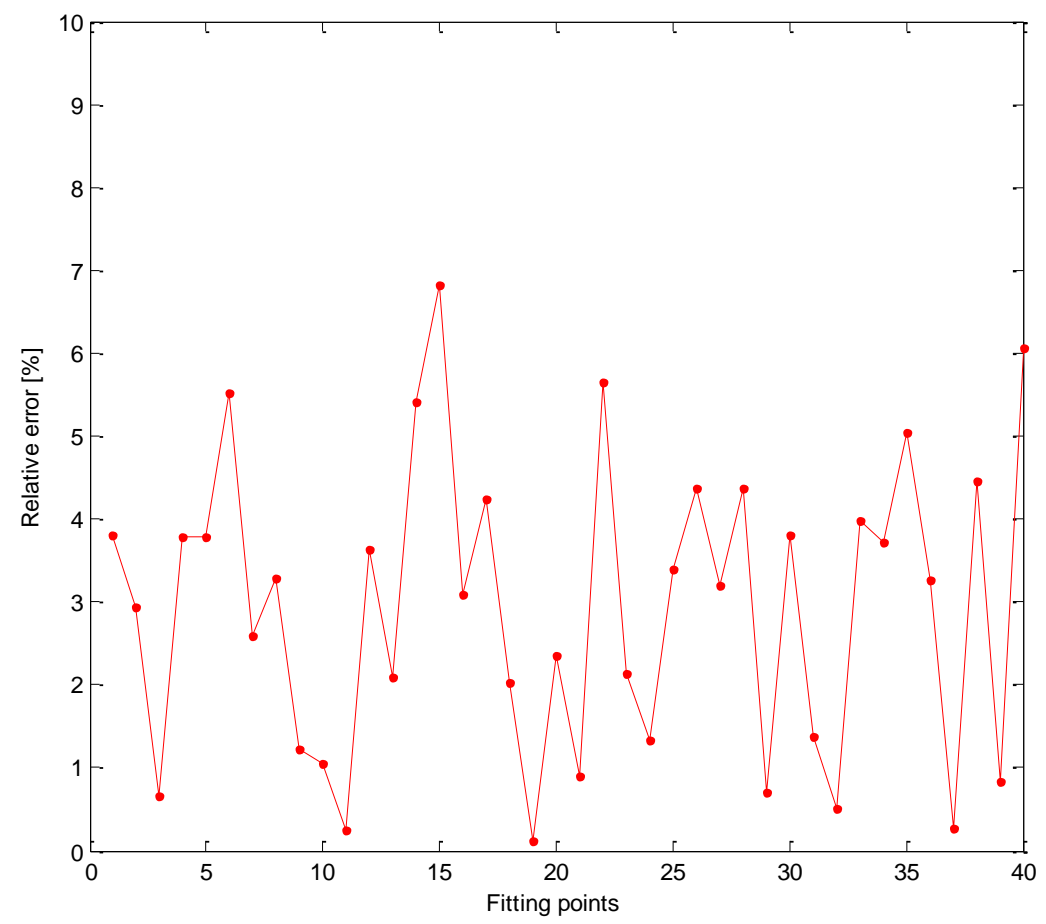

Figure 101 Relative error of $W_{\text {eva,con }}$ 


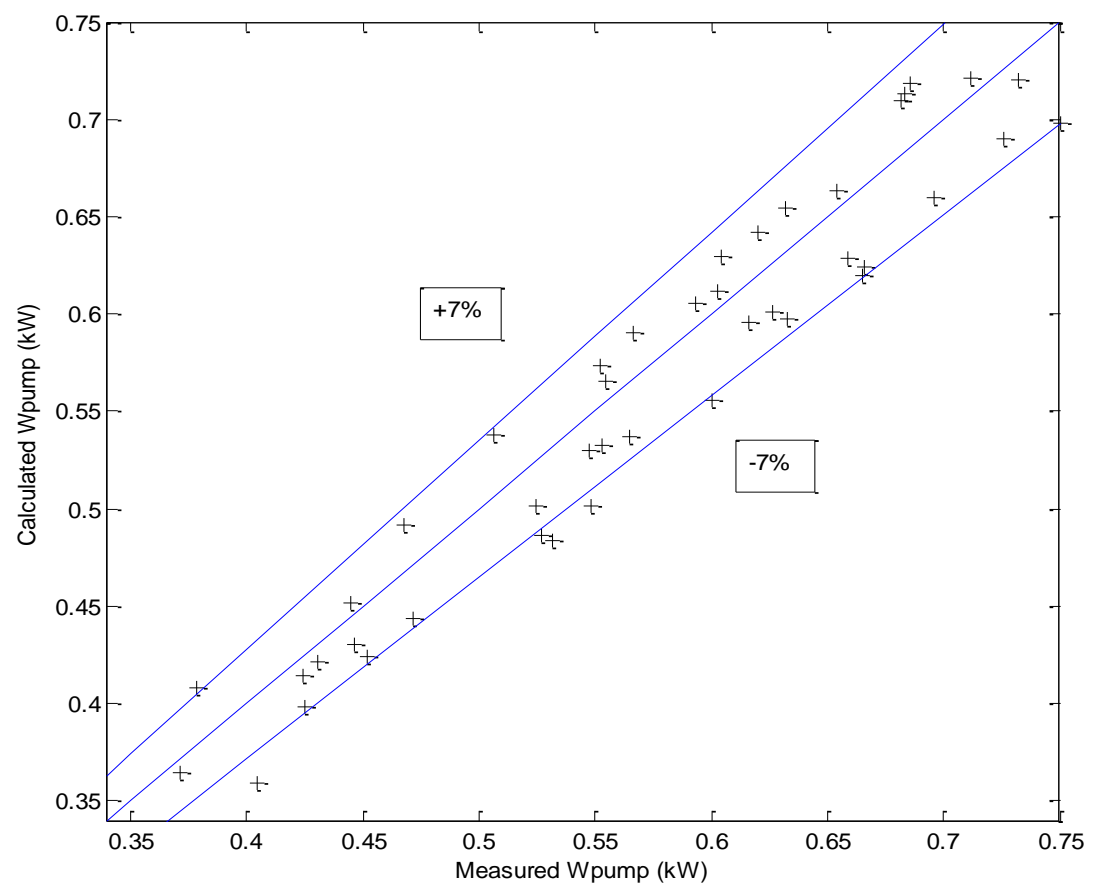

Figure 102 Fitting results of $W_{\text {pump }}$

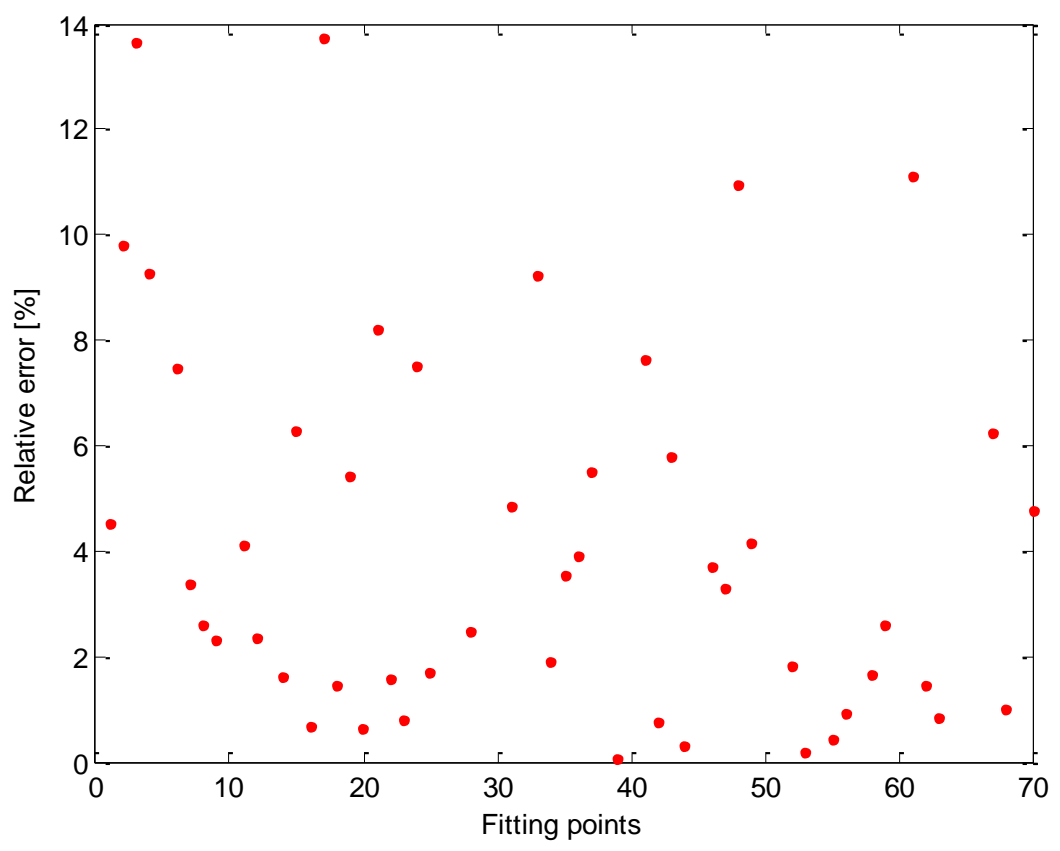

Figure 103 Relative error of $W_{\text {pump }}$ 
From Figures 99, 101 and 103, it can be found that the majority of relative errors of power consumption model of fans and liquid pump are within $\pm 6 \%, \pm 5 \%$ and $\pm 10 \%$ which indicates the accuracy of developed model is enough for optimization purpose.

\subsubsection{Constraints}

The physical constraints are illustrated before when ELM based optimization problems are formulated. Here, the interaction effects of two components are presented.

The interactions of two components can be derived based on the energy conservation of refrigerant in the cycle. For instance, the refrigerant will absorb energy during generator, evaporator, compressor and liquid pump. And the absorbed energy will be transferred and expended during condenser. Hence, the energy balance equation can be written as

$$
\dot{Q}_{\text {con }}=\dot{Q}_{\text {gen }}+\dot{Q}_{\text {eva }}+\dot{W}_{\text {com }}+\dot{W}_{\text {pump }}
$$

where

$$
\dot{Q}_{c o n}=\left(\dot{m}_{p}+\dot{m}_{s}\right) \Delta h_{c o n, r}
$$

And

$$
\begin{gathered}
\dot{Q}_{\text {gen }}=\dot{m}_{p} \Delta h_{\text {gen }, r} \\
\dot{Q}_{\text {eva }}=\dot{m}_{s} \Delta h_{\text {eva }, r} \\
\dot{W}_{c o m}=\dot{m}_{s} \Delta h_{\text {eva }, r} \\
\dot{W}_{\text {pump }}=\dot{m}_{p} \Delta h_{\text {pump }, r}
\end{gathered}
$$

The $\Delta h$ represents the enthalpy difference between inlet and outlet of corresponding components. And the enthalpy can be obtained as

$$
h=f(P, T)=a_{1}+a_{2} P+a_{3} T+a_{4} P T
$$

where the $a_{1}$ to $a_{4}$ are constants determined by curve fitting.

\subsubsection{Variables categorization}

With the purpose of reducing the dimension of variables as well as simplifying the computational process, variables are classified according to their properties and the relative variables are to be replaced by variables with same properties. The detailed classification results are shown as: 
- Uncontrollable variables: The distinct feature of uncontrollable variables is that they are determined either by surrounding circumstance or by requirement of user. Hence, ambient temperature, $T_{a m b}$, indoor air temperature, $T_{i n}$, and cooling capacity, $Q_{e}$, are regarded as uncontrollable variables and their values are fixed at each computational process. Additional, the ambient temperature, $T_{a m b}$, is the most crucial one as the rest variables, $T_{i n}$ and $Q_{e}$, are influenced by it.

- Independent variables: The character that makes independent variables distinguished from others are that there is no extra restriction on selection of independent variables as long as the constraint limits are met. In this case, the most apparently selections for independent variables are $f_{\text {com }}, f_{\text {con,fan }}, f_{\text {eva,fan }}, f_{\text {pump }}$ and $A_{v}$ since these variables are straightly determined by compressor, fans of heat exchanger, liquid pump and EEV, respectively. In order to avoid the curse of dimensionality and improve results veracity, the $f_{c o m}, P_{e v a, i n}, P_{c o n, i n}, P_{e, p, i n}$ and $T_{\text {con,out }}$ are assigned to be independent variables.

- Dependent variables: Different from independent variables, dependent variables can be obtained via computing interaction and constrained relationship with independent variables. Then, the dependent variables can be employed and be participated in solving the optimization problems.

Figure 104 shows the classification result of state variables. 


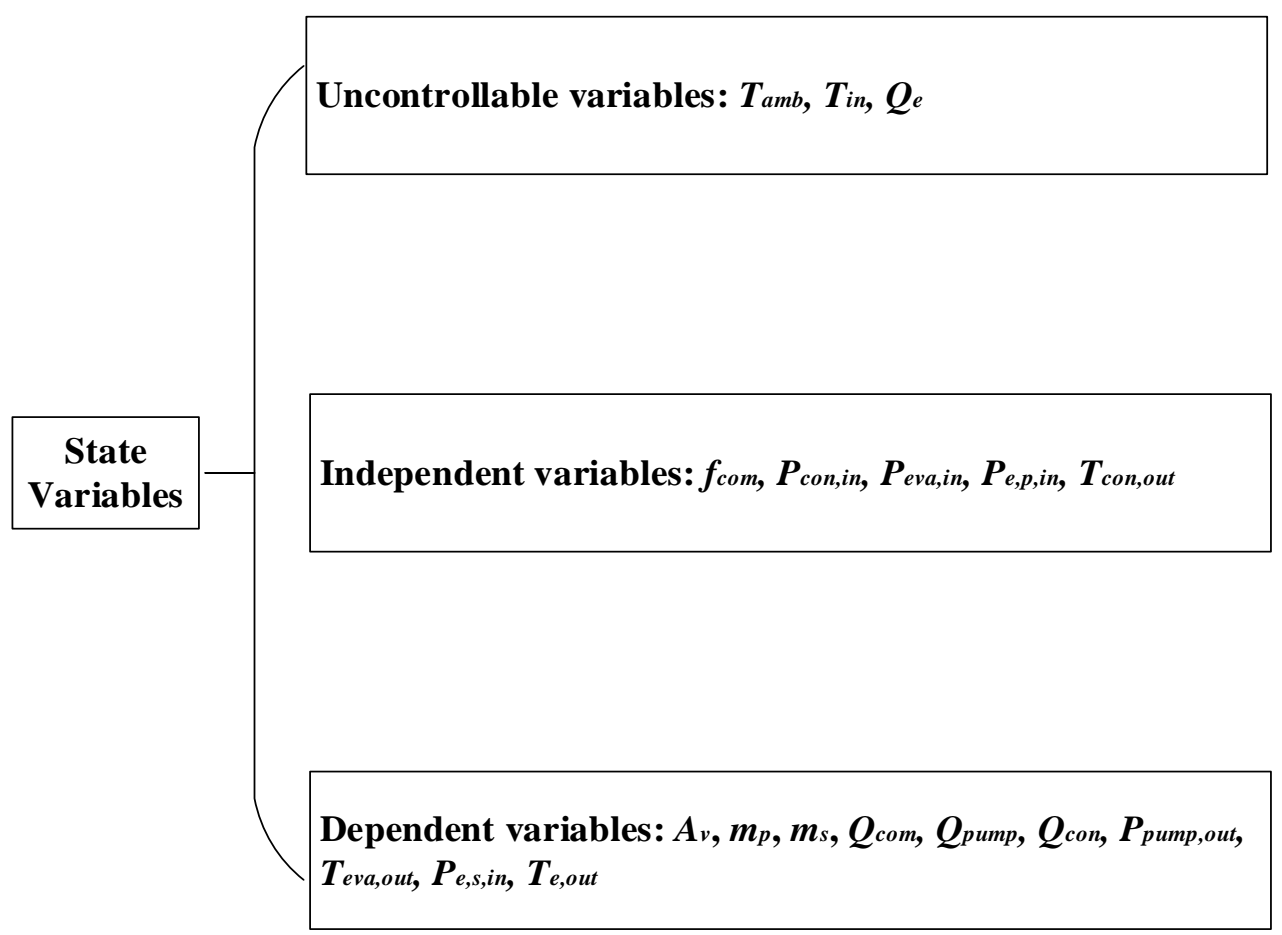

Figure 104 Classification result

\subsection{Optimization algorithm}

\subsubsection{Implementation of PSOGA}

A particle swarm optimization algorithm integrated with evolutionary operators from genetic algorithm (PSOGA) is proposed to solve the constrained nonlinear optimization problem. This combined algorithm shares the high efficiency character of PSO and has larger possibility to escape from local minimum with the evolutionary operators mixed in. Additional, the proposed PSOGA can be divided into four steps: encoding, fitness function construction, iteration and termination. The detailed procedure will be concisely illustrated as follow.

Encoding: As mentioned before, during encoding process, each variable is changed into a binary string following gray coding fashion since it can overcome potential representative bias. And a chromosome is built by all these strings. Normally, the more precise the control aims to achieve, the longer strings is required. However, the longer strings also mean higher computational cost. Therefore, it is a trade-off between efficiency and precision. After encoding, the generated chromosomes are employed in the algorithm to approach the optimal chromosomes. To obtain the optimal solution of real values, it is 
necessary to decode the optimal chromosomes. The whole generation ought to manage to cover as entire candidates region as possible.

Fitness function construction: With the coded variables settled, the fitness function is constructed to evaluate the performance of each individual chromosome. The reciprocal of objective function is taken as fitness function because the maximum fitness value is obtained when the total energy consumption is at its minimum. In case of the constraints being violated, the penalty function is also constructed to compensate the violation cost to fitness function. Under such idea, the fitness function is expressed as:

$$
\text { Fitness }=\frac{1}{\left(\dot{W}_{c o m}+\dot{W}_{e v a, f a n}+\dot{W}_{c o n, f a n}+\dot{W}_{p u m p}+K_{t o t a l}\right)}
$$

Since the generator is assumed to be powered by low-grade heat energy, such as solar, geothermal and waste heat energy, it is not accountable for the total power consumption of the system. Additional, the penalty function $K_{\text {total }}$ is expanded in detail as:

$$
K_{\text {total }}=\left\{\begin{array}{ccc}
0 & \text { if } & \sum_{i=1}^{N} K_{i}=0 \\
t+\sum_{i=1}^{N} K_{i} & \text { if } & \sum_{i=1}^{N} K_{i} \neq 0
\end{array}\right.
$$

where $t$ is an user-assigned constant and is usually three times of the biggest contributor, which in this case is the compressor. And $K_{i}, \mathrm{i}=1, \ldots, N$ are the state penalty functions of each individual. For instance, the penalty function of condenser fan frequency is shown as

$$
K_{c o n, f a n}=\left\{\begin{array}{cc}
0 & \text { if } f_{c o n, f a n, \text { min }} \leq f_{c o n, f a n} \leq f_{c o n, f a n, \text { max }} \\
\left|\left(f_{c o n, f a n, \min }-f_{c o n, f a n}\right) / f_{c o n, f a n}\right| & \text { if } f_{c o n, f a n, \text { min }}>f_{c o n, f a n} \\
\left|\left(f_{c o n, f a n, \max }-f_{c o n, f a n}\right) / f_{c o n, f a n}\right| & \text { if } f_{c o n, f a n}>f_{c o n, f a n, \max }
\end{array}\right.
$$

By calculating the violation function, the magnitude of fitness of each individual chromosome is shifted and updated for range predefined for better control over evolution.

Iteration: In iteration, the individual is arranged according to their own fitness values, the higher the fitness value is; the more front it is located. The higher half part directly enters the next generation while the two evolutionary operators is applied on lower half part, namely crossover and mutation are borrowed from GA to help improve the performance 
of PSO. The roulette wheel selection method is applied. Besides, crossover operator follows the single point manner and single bit pattern for mutation operator. The crossover points and mutation bit are all randomly chosen.

Termination: The PSOGA algorithm won't terminate unless at least one of the criterion described below is met:

1. The iteration number reaches the predetermined maximum number.

2. The variation of optimal fitness value is within predefined range over several steps. By applying evolutionary operators to individuals with lower fitness value, hopefully, the fitness condition may be improved among their off-springs. Therefore, the proposed PSOGA may require a little bit more computational cost than the traditional PSO, the possibility of finding feasible solution is increase with evolutionary operators involved. In each generation, the eligible individual with maximum fitness value is stored as optimal solutions waiting to be compared with last generation. This manner will reduce the probability of losing good chromosome.

\subsubsection{Scheme operation steps}

The proposed PSOGA optimization approach for hybrid system is summarized and illustrated below:

1st: Parameters identification. The unknown coefficients of previous energy consumption models of relative component are determined by experimental data;

2nd: Obtain the data of uncontrollable variables by direct measurement and calculation;

3rd: PSOGA parameter initialization. The initialized parameters include: size of population, maximum generation number, length of chromosome, value of two cognitive and social factors, value of inertia weight, maximum velocity of particle, dimensional number of searching space and probabilities of genetic operators (such as crossover and mutation).

4th: Operate the PSOGA algorithm.

5th: Store the chromosome with highest fitness value in each generation and replace the global best location with chromosome currently having highest fitness value. Update the velocity and position of particle according to PSO algorithm. Return to step 4 and step 5 until predetermined termination criterion is met. 
6th: The individual with highest fitness magnitude over others within entire generations is selected as optimal solution and is employed in the calculation of system total energy consumption.

The flow chart shown in Figure 105 is used to illustrate specifically the PSOGA algorithm.

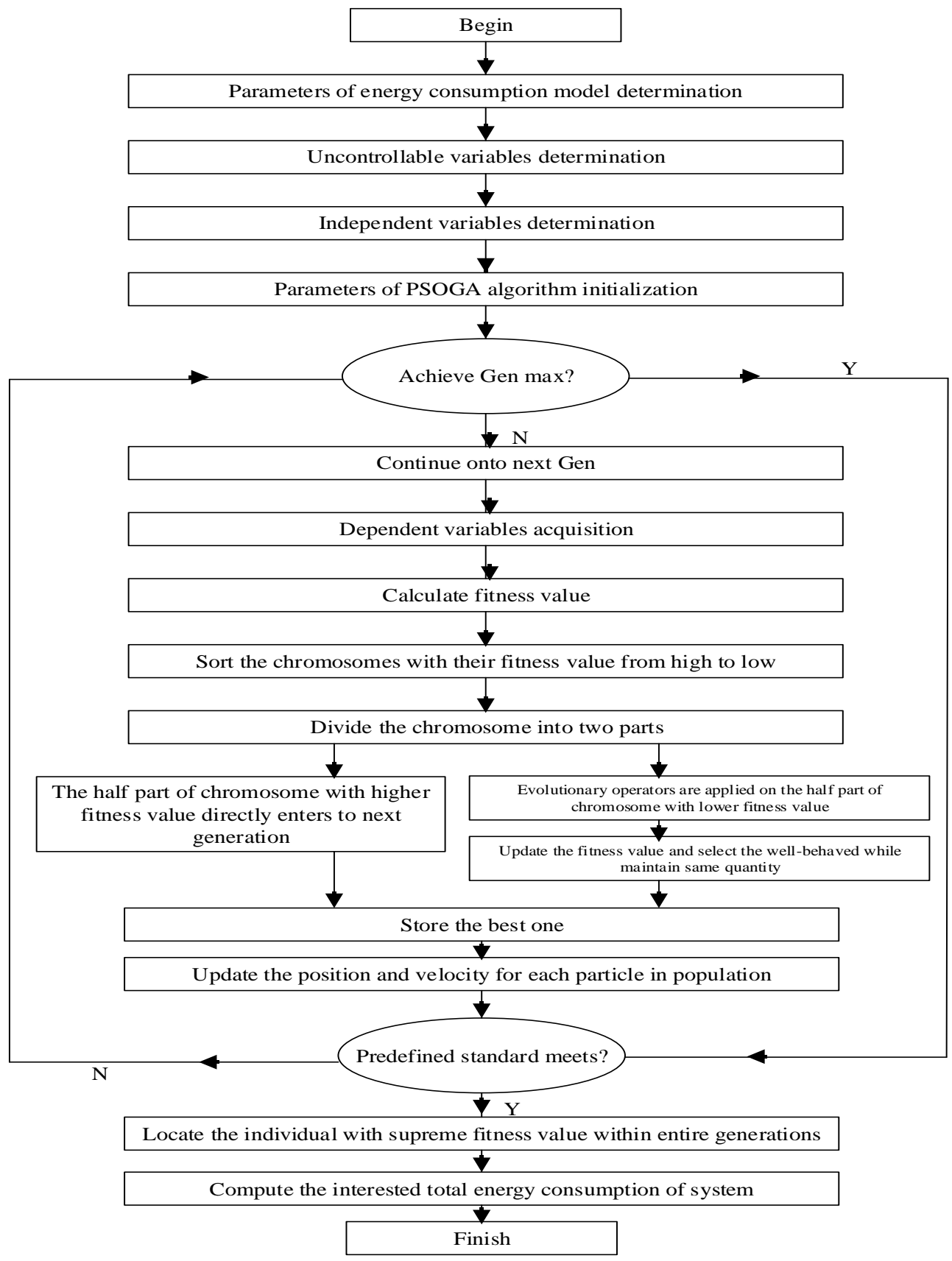

Figure 105 Flow chart of PSOGA optimization approach 


\subsection{Experiment results and discussion}

The experiments are conducted on the research-designed hybrid system platform. In order to simulate the cooling load in practice, the temperature from 6am to 20pm of one single day is recorded. With indoor temperature set at $22^{\circ} \mathrm{C}$, the higher temperature is, the larger demand of cooling capacity is. As the temperature is proportion to the cooling load, the variation trend of temperature can be directly transferred into that of cooling load. The unknown parameters contained in energy models are determined via curve fitting of 500 groups of experiment data under different operating conditions (different compressor, heat exchangers fan and liquid pump frequencies, respectively). And the physical constraints are listed in Table 6.

Table 6. Physical bounds of parameters

\begin{tabular}{lll}
\hline Parameters & Lower limit & Upper limit \\
\hline$f_{\text {con }, f a n}$ & $10 \mathrm{~Hz}$ & $50 \mathrm{~Hz}$ \\
$f_{\text {eva,fan }}$ & $10 \mathrm{~Hz}$ & $40 \mathrm{~Hz}$ \\
$T_{\text {amb }}$ & $27^{\circ} \mathrm{C}$ & $34^{\circ} \mathrm{C}$ \\
$P_{\text {con }}$ & $8 \mathrm{bar}$ & $12 \mathrm{bar}$ \\
$P_{\text {eva }}$ & $2.5 \mathrm{bar}$ & $4.5 \mathrm{bar}$ \\
$P_{\text {gen }}$ & $10 \mathrm{bar}$ & $25 \mathrm{bar}$ \\
$A_{v}$ & $30 \%$ & $60 \%$ \\
$f_{\text {com }}$ & $30 \mathrm{~Hz}$ & $50 \mathrm{~Hz}$ \\
$\dot{m}_{p}$ & $0.016 \mathrm{~kg} / \mathrm{s}$ & $0.06 \mathrm{~kg} / \mathrm{s}$ \\
$\dot{m}_{s}$ & $0.004 \mathrm{~kg} / \mathrm{s}$ & $0.012 \mathrm{~kg} / \mathrm{s}$ \\
$f_{\text {pump }}$ & $20 \mathrm{~Hz}$ & $60 \mathrm{~Hz}$ \\
\hline
\end{tabular}

The corresponded cooling load trend from 6am to 20pm is shown in Figure 106. 


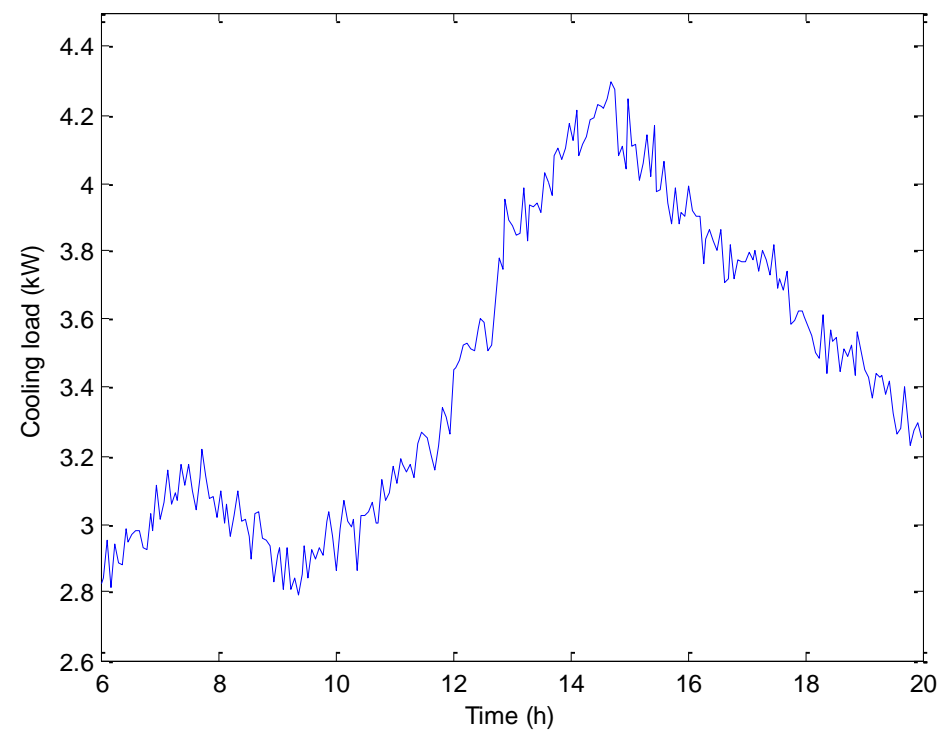

Figure 106 Corresponded cooling load trend

To simplify the calculation for both simulation and testing, the mean cooling load value is applied within the 1 hour interval from $6 \mathrm{am}$ to $20 \mathrm{pm}$. The energy consumptions under both PSOGA and traditional hysteresis control (or known as on-off control) strategy are displayed for comparison. And the corresponding result is shown in Figure 107.

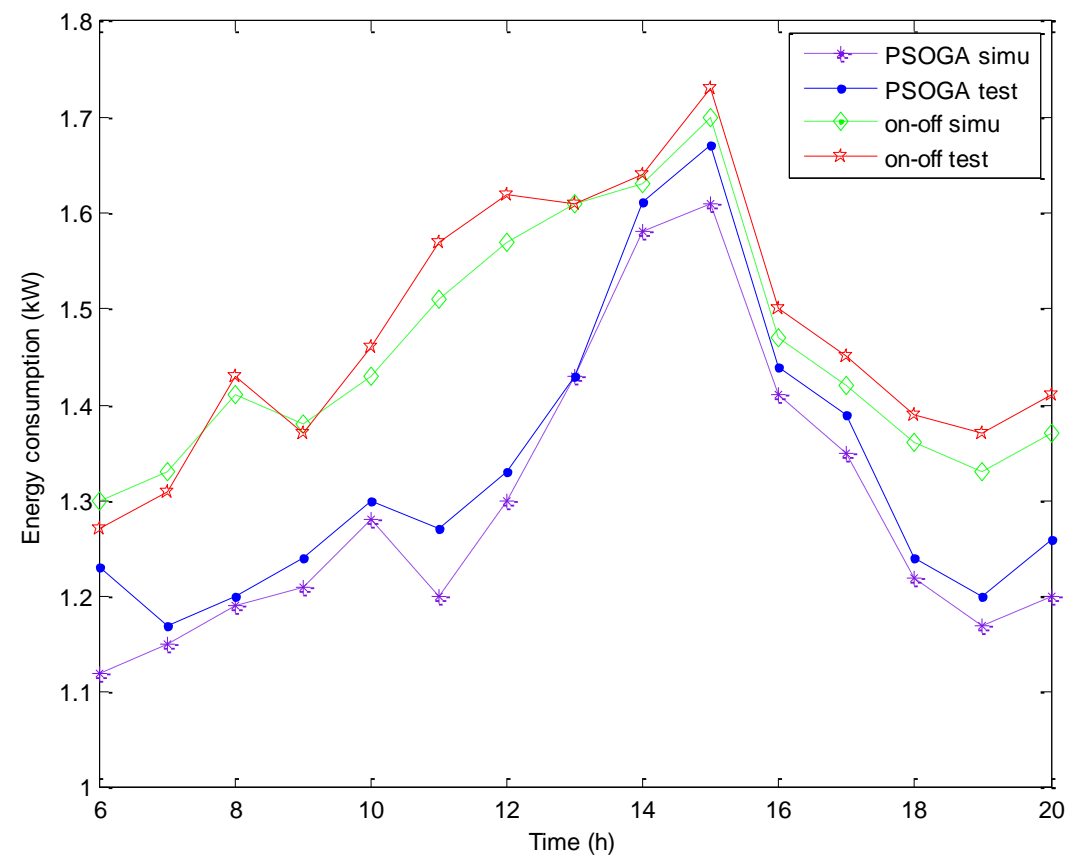

Figure 107 Energy consumptions under different optimization approach 
Moreover, the operation conditions for one chosen time points (namely 12:00) between hysteresis control and PSOGA are listed in Table 7.

Table 7. Operation conditions for selected time point

\begin{tabular}{lll}
\hline Variables & On-off & PSOGA \\
\hline$f_{\text {con,fan }}$ & $35 \mathrm{~Hz}$ & $30 \mathrm{~Hz}$ \\
$f_{\text {eva fan }}$ & $40 \mathrm{~Hz}$ & $35 \mathrm{~Hz}$ \\
$T_{\text {amb }}$ & $33^{\circ} \mathrm{C}$ & $33^{\circ} \mathrm{C}$ \\
$P_{\text {con }}$ & $10 \mathrm{bar}$ & $9.7 \mathrm{bar}$ \\
$P_{\text {eva }}$ & $3.2 \mathrm{bar}$ & $3.5 \mathrm{bar}$ \\
$P_{\text {gen }}$ & $18 \mathrm{bar}$ & $21 \mathrm{bar}$ \\
$A_{v}$ & $45 \%$ & $55 \%$ \\
$f_{\text {com }}$ & $45 \mathrm{~Hz}$ & $38 \mathrm{~Hz}$ \\
$\dot{m}_{p}$ & $0.043 \mathrm{~kg} / \mathrm{s}$ & $0.052 \mathrm{~kg} / \mathrm{s}$ \\
$\dot{m}_{s}$ & $0.011 \mathrm{~kg} / \mathrm{s}$ & $0.009 \mathrm{~kg} / \mathrm{s}$ \\
$f_{\text {pump }}$ & $35 \mathrm{~Hz}$ & $45 \mathrm{~Hz}$ \\
\hline
\end{tabular}

From the operation conditions of specific point, it can be observed that the compressor speed under on-off control is higher than that of PSOGA, while the pump speed of on-off is lower than that of PSOGA. The overall energy consumption difference is attributed by these two components. The PSOGA tends to better utilize the ejector, which requires more energy being distributed to pump. The PSOGA is able to find the best solution within pre-defined criteria while the on-off can just meet the target regardless of cost. The optimized result can be feedback to the control system and be compared with the real operation conditions, furthermore, the feed-back control will take the advantage of relative error to adjust the corresponding parameter of the system.

From Figure 106, it can be seen that the energy consumption with PSOGA is lower than that with hysteresis control which demonstrates the energy conservation effect of presented approach. Moreover, the almost all the simulation result of energy consumption is lower than the experiment result. Additional, the average deviation among simulation and experiment results is $4.73 \%$ which is calculated by $\Delta=\frac{1}{n} \sum_{1}^{n}\left(\frac{\left|W_{\text {simu }, n}-W_{\text {test }, n}\right|}{W_{\text {test }, n}}\right) \times 100 \% \quad n=1,2, \ldots 15$ and the max deviation is $9.76 \%$ which happens at 6 am and is calculated by $\Delta_{\max }=\frac{\left|W_{\text {simu }}-W_{\text {test }}\right|}{W_{\text {test }}} \times 100 \%$. The reason might be attributed to the errors of measuring instruments and of energy models. Whereas, it is 
tolerable towards such degree of error in practice and the performance of PSOGA is sufficiently satisfying. To further investigate the energy saving effect under different ambient temperature, the energy consumption and corresponding saving amount are rearranged with variation of ambient temperature. And the result is described in Figure 108.

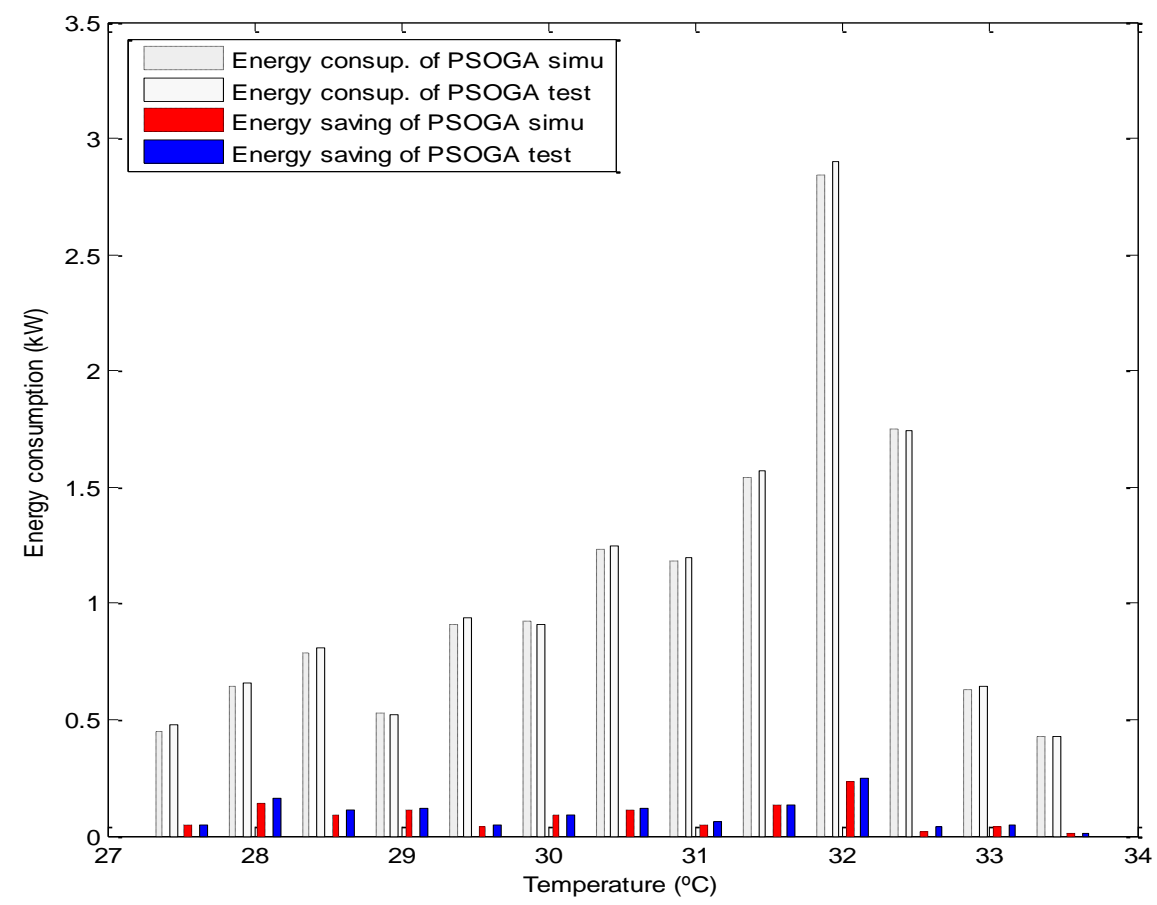

Figure 108 Energy consumption and saving effect with variation of ambient temperature

It can be found that the energy saving effects achieves its highest value at $32^{\circ} \mathrm{C}$ with $11.7 \%$ which is obtained through $\frac{W_{\text {saving }}}{W_{\text {consup }}} \times 100 \%$, and the average value of saving effects over the entire temperature is $7.36 \%$ via $\frac{1}{n} \sum_{1}^{n} \frac{W_{\text {saving }, n}}{W_{\text {consup }, n}} \times 100 \% \quad n=1,2, \ldots 13$.

From the optimized energy consumption result with ELM and PSOGA, it can be found that the PSOGA achieve more energy saving effect (overall 7.36\%) than ELM (5.83\%). However, the processing time of ELM is shorter than that of PSOGA due to the high convergence speed character of ELM. Therefore, it is a trade-off between time and accuracy when selecting the appropriate optimization method. 


\subsection{Summary}

In this chapter, a centralized optimization approach is proposed to help optimize the performance of the hybrid ejector-based air conditioning system based on derived components models. The objective of this optimization problem is to minimize the total energy consumption while be satisfied with physical constraints of devices, interactions between components, ambient conditions and required cooling capacities. A particle swarm optimization algorithm integrated with evolutionary operators from genetic algorithm is presented along with corresponding solution procedure to efficaciously and rapidly achieve the optimal set value under different working situations. Both the simulation and experiment results have testified that the set points obtained via PSOGA do generate more reduction in energy consumption when compared with that of conventional on-off control method. Additional, it is shown that the average energy saving effects over a common weekday from $6 \mathrm{am}$ to $20 \mathrm{pm}$ is $7.36 \%$. Last but not the least, the proposed approach is confirmed to be effective enough due to the fact that the average deviation among simulation and experiment is $4.73 \%$. 


\section{Chapter 8 Conclusion and Future Works}

\subsection{Conclusion}

In this thesis, several optimization techniques for the hybrid system's modeling and operational aspects prediction have been established. The overview and background of ejector refrigeration system have been presented in Chapter 1. The fundamental knowledge of artificial neural network along with several intelligent modeling approaches, such as the back-propagation, the radial basis function, the support vector machine and the extreme learning machine, and several intelligent optimization algorithms, including genetic algorithm and particle swarm optimization, have been illustrated in Chapter 2. Chapter 3 concentrates on the performance evaluation of system under different operation conditions. Comparison between hybrid mode and general mode is made. The main system's components modeling are proposed in Chapter 4. The fouling effect is taken into account to modify the original hybrid models. Chapter 5 introduces an innovative artificial neural network modeling method for hybrid system. The network is trained with the ELM and the BP, RBF and SVM for comparison purpose. The results indicate the robustness of proposed ANN model against the unpleasant disturbance and satisfying prediction of system information. In Chapter 6, a SLFN classifier is developed to identify the system stability in preparation for the application of presented optimization method towards hybrid system. By establishing the relationship within measured controllable variables and system energy consumption, the optimization problem is figured out via an exhaustive grid search manner. In Chapter 7, the optimization problem is built in centralized fashion and is worked out with evolutionary operators integrated particle swam optimization method. The optimal set points are obtained with varying cooling capacities and ambient conditions.

\subsection{Future works}

The intention of this research work is to establish several systematic methodologies to control the operation of a hybrid ejector-based air-conditioning system with minimum power consumption under desirable indoor environment quality requirement. However,

there still remains a lot of works to do to realize the hybrid system online optimization in practice, including: 
1. In Chapter 5, the brief comparison between ELM and other five different neural networks is made to indicate the outperformance of proposed ELM. There still remains certain aspect to study. For instance, [123] proposed a functional-link net with random vector and augmented input that can replace the hidden layer. Therefore, several ANN models will be selected for more explicit comparison [124].

2. The proposed optimization approaches are of centralized manner, it is interesting to apply decentralized optimization method to the proposed system $[125,126]$. Although the global performance of decentralized optimization is probably less than that of centralized one, the computational speed will be faster than that of centralized way. Therefore, it is a trade-off between efficiency and performance. It will be a challenge about how to isolate each component in order to formulate subsystems for sub-optimal problem [127].

3. The robustness analysis of both centralized and decentralized methods reveals wide realm to be investigated in development of hybrid system optimization. The sensitive extent of coefficients of components will be of interest, for the stability of system to be against disturbance [128]. For instance, the determination of feasible limits for parameter variation within proposed component model.

4. Since ejector is the key component in hybrid system, the performance evaluation of it under different geometric parameters is needed. The effect of nozzle exit position, nozzle angle, and diffusion angle is of interest [129, 130]. More than one ejector can be installed in the system either in cascade form or parallel pattern $[131,132]$. The simulation can be done on CFD first and then the lab scale experiment can be carried out.

5. Due to the good performance of ELM in system modeling, the variants of ELM shall have good potential. Since ELM tends to require more hidden neurons than conventional tuning based algorithm, it is necessary to find a novel learning algorithm that can achieve more compact network architecture and require less number of hidden neurons than common ELM [133]. Differential evolution algorithm is known for its ability and efficiency to locate global optimum, therefore, it is interesting to apply differential evolution (maybe some modification 
is needed) to search for the optimal input weights and hidden biases rather than randomly selection.

6. The optimizations proposed are single objective problem, but in practice, multiple objectives are often encountered, such as requirement of relative humidity and $\mathrm{CO}_{2}$ density. Thus, multi-objective optimization is needed in the future [134, 135]. The objectives may conflict each other in practice, and the Pareto optimal solutions is the suitable choice [136]. How to construct the 'Pareto front' in order to locate the Pareto optimal solutions is remained to be dealt with. 


\section{Appendix A An Hourly Sample Data of $Q_{d e}$}

Table 8. Sample data of $\mathrm{Q}_{\mathrm{de}}$

\begin{tabular}{|l|l|}
\hline Time & $\mathrm{Q}_{\mathrm{de}}(\mathrm{kW})$ \\
\hline $6: 00$ & 2.82 \\
\hline $6: 05$ & 2.84 \\
\hline $6: 10$ & 2.87 \\
\hline $6: 15$ & 2.83 \\
\hline $6: 20$ & 2.86 \\
\hline $6: 25$ & 2.85 \\
\hline $6: 30$ & 2.90 \\
\hline $6: 35$ & 2.93 \\
\hline $6: 40$ & 2.86 \\
\hline $6: 45$ & 2.98 \\
\hline $6: 50$ & 3.04 \\
\hline $6: 55$ & 3.01 \\
\hline $7: 00$ & 3.12 \\
\hline
\end{tabular}




\section{Appendix B Levenberg-Marquart method}

The iteration direction can be obtained via solving the following equation:

$$
\left(J^{(k)^{T}}(c) J^{(k)}(c)+{ }^{(k)} I\right) P^{(k)}(c)=J^{(k)^{T}}(c) r^{(k)}(c)
$$

where

$$
r(c)=\left[\begin{array}{llll}
r_{1}(c) & r_{2}(c) & \cdots & r_{N}(c)
\end{array}\right]^{T}
$$

To ensure the character of positive definite of left side as well as to reduce the effect of singularity, the addition of ${ }^{(k)} I$. And the $J^{(k) T}$ is the Jacobian matrix which can be obtained as

$$
J=\left[\begin{array}{cccc}
\frac{\partial r_{1}}{\partial c_{1}} & \frac{\partial r_{1}}{\partial c_{2}} & \cdots & \frac{\partial r_{1}}{\partial c_{n}} \\
\frac{\partial r_{2}}{\partial c_{1}} & \frac{\partial r_{2}}{\partial c_{2}} & \cdots & \frac{\partial r_{2}}{\partial c_{n}} \\
\vdots & \vdots & \ddots & \vdots \\
\frac{\partial r_{N}}{\partial c_{1}} & \frac{\partial r_{N}}{\partial c_{2}} & \cdots & \frac{\partial r_{N}}{\partial c_{n}}
\end{array}\right]
$$

With the iteration direction obtained, then the coefficient can be updated as

$$
c^{(k+1)}=c^{(k)}+P^{(k)}
$$

The iteration process won't terminate unless the predefined criterion is reached which is

$$
\left|c^{(k+1)} \quad c^{(k)}\right|<
$$

And in this case, the is selected as $1 \times 10^{-7}$ 


\section{Appendix C Penalty Calculation of constraints}

Penalty of evaporator fan frequency:

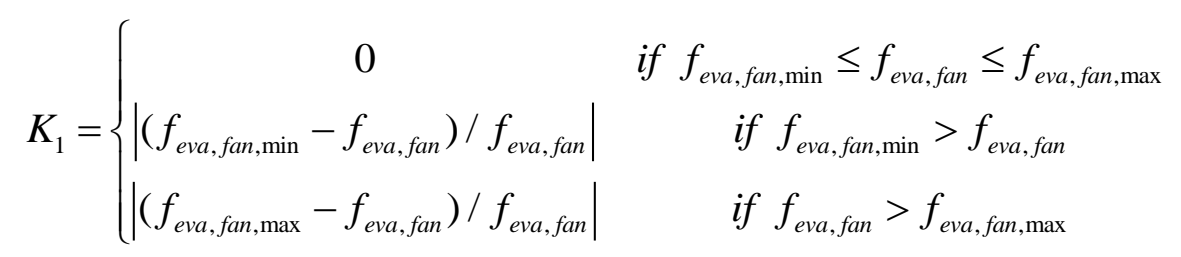

Penalty of condensing saturated temperature:

$$
K_{2}=\left\{\begin{array}{cc}
0 & \text { if } T_{\text {con,out }} \leq T_{\text {con,sat }} \leq T_{\text {con, in }} \\
\left|\left(T_{\text {con, in }}-T_{\text {con,sat }}\right) / T_{\text {con,sat }}\right| & \text { if } T_{\text {con, in }}<T_{\text {con, sat }}
\end{array}\right.
$$

Penalty of evaporating saturated temperature:

$$
K_{3}=\left\{\begin{array}{cl}
0 & \text { if } T_{\text {eva,sat }} \leq T_{\text {indoor }} \\
\left|\left(T_{\text {eva,sat }}-T_{\text {indoor }}\right) / T_{\text {eva,sat }}\right| & \text { if } T_{\text {indoor }}<T_{\text {eva,sat }}
\end{array}\right.
$$

Penalty of ejector back pressure:

$$
K_{4}=\left\{\begin{array}{cl}
0 & \text { if } P_{\text {back }} \leq P_{\text {critical }} \\
\left|\left(P_{\text {back }}-P_{\text {critical }}\right) / P_{\text {critical }}\right| & \text { if } P_{\text {back }}>P_{\text {critical }}
\end{array}\right.
$$

Penalty of compression operation frequency:

$$
K_{5}=\left\{\begin{array}{cc}
0 & \text { if } 30 \leq f_{\text {com }} \leq 50 \\
\left|\left(30-f_{\text {com }}\right) / f_{\text {com }}\right| & \text { if } f_{\text {com }}<30 \\
\left|\left(50-f_{\text {com }}\right) / f_{\text {com }}\right| & \text { if } f_{\text {com }}>50
\end{array}\right.
$$

Penalty of total refrigerant mass flow rate:

$$
K_{7}=\left\{\begin{array}{cc}
0 & \text { if } \dot{m}_{r, \text { min }} \leq \dot{m}_{r} \leq \dot{m}_{r, \max } \\
\left|\left(\dot{m}_{r, \min }-\dot{m}_{r}\right) / \dot{m}_{r}\right| & \text { if } \dot{m}_{r, \min }>\dot{m}_{r} \\
\left|\left(\dot{m}_{r, \max }-\dot{m}_{r}\right) / \dot{m}_{r}\right| & \text { if } \dot{m}_{r}>\dot{m}_{r, \max }
\end{array}\right.
$$

Penalty of EEV Opening Percentage:

$$
K_{8}\left\{\begin{array}{cl}
0 & \text { if } 0.3 \leq A_{v} \leq 0.6 \\
\left|\left(A_{v}-0.3\right) / A_{v}\right| & \text { if } A_{v}<0.3 \\
\left|\left(A_{v}-0.6\right) / A_{v}\right| & \text { if } A_{v}>0.6
\end{array}\right.
$$




\section{Author's Publications}

1. Wang Hao, Cai Wenjian, Wang Youyi, Yan Jia and Wang Lei, Experimental Study of the Behavior of a Hybrid Ejector-based Air-conditioning System with R134a. Energy Conversion and Management, v112, p31-40, 2016.

2. Wang Hao, Cai Wenjian and Wang Youyi, Modeling of a Hybrid Ejector Air Conditioning System using Artificial Neural Networks, Energy Conversion and Management, v127, p11-24, 2016.

3. Wang Hao, Cai Wenjian and Wang Youyi, Optimization of a Hybrid Ejector airconditioning System with PSOGA, Applied Thermal Energy, v112, pp1474-1486, 2017.

4. Wang Hao, Cai Wenjian and Wang Xinli, Experimental Analysis of a Hybrid Ejectorbased Air-conditioning System Using R134a, in 2014 7th International Conference on Information and Automation for Sustainability, ICIAfS. Colombo, Sri Lanka, Dec 2224, 2014. Selected as the Outstanding Conference Paper. 


\section{Reference}

[1] Liao Yuhang, "Performance analysis on a solar-driven air-cooled ejector refrigeration system for air-conditioning using ammonia as refrigerant," Applied Mechanice and Materials, vol. 501-504, pp. 2282-2287, 2014.

[2] R'mierciew, K, "Experimental investigation of solar driven ejector air-conditioning system," Energy and Buildings, vol. 80, pp. 260-267, 2014.

[3] Bin-Juine Huang, "Performance test of solar-assisted ejector cooling system," International Journal of Refrigeration, vol. 39, pp. 172-185, 2014.

[4] Sumeru and Martin L, "Energy savings in air conditioning system using ejector: an overview," Applied Mechanics and Materials, vol. 493, pp. 93-98, 2014.

[5] Radermacher Reinhard, " Integrating alternative and conventional cooling technologies," ASHRAE Journal, vol. 49, pp. 28-35, 2007.

[6] Jiautheen P.B and Annamalai M, "Review on ejector of vapor jet refrigeration system," International Journal of Air-conditioning and Refrigeration, vol. 22, pp. 143-173, 2014.

[7] Fang Liu and Groll E.A, "Study of ejector efficiencies in refrieration cycles," Applied Thermal Engineering, vol. 52, pp. 360-370, 2013.

[8] Al-Alili.A and Yunho Hwang, "Review of solar thermal air conditioning technologies," International Journal of Refrigeration, vol. 39, pp. 4-22, 2014.

[9] Khaliq.A, "Performance analysis of a waste-heat-powered thermodynamic cycle for multi-effect refrigeration," International Journal of Energy Research, vol. 39, pp. 529-542, 2015.

[10] Pounds D.A, Dong JM, Cheng P and Ma H B, "Experimental investigation and theoretical analysis of an ejector refrigeration system," International Journal of Thermal Sciences, vol. 67, pp. 200-209, 2013.

[11] Zhu Yinhai and Jiang Peixue, "Hybrid vapor compression refrigeration system with an integrated ejector cooling cycle," International Journal of Refrigeration, vol. 35, pp. 68-78, 2012.

[12] Yan Jia, Cai Wenjian and Li Yanzhong, "Geometry parameters effect for aircoolied ejector cooling system with R134a refrigerant," Renewable energy, vol. 35, pp. 155-163, 2012.

[13] Yan Jia and Cai Wenjian, "Area ratio effects to the performance of air-cooled ejector refrigeration cycle with R134a refrigerant," Energy Conversion and Management, vol. 53, pp. 240-246, 2012.

[14] Chen Jianyong, Havtun Hans and Palm Bjorn, "Investigation of ejectors in refrigeration system: Optimum performance evaluation and ejector area ratios perspectives," Applied Thermal Engineering, vol. 64, pp. 182-191, 2014.

[15] Wang F, Shen S Q and Li D Y, "Evaluation on environment-friendly refrigerants with similar normal boiling points in ejector refrigeration system," Heat and Mass Transfer, vol. 51, pp. 965-972, 2014.

[16] Besagni Giorgio, Mereu Riccardo, Di Leo, Giuseppe and Inzoli Fabio, "A study of working fluids for heat driven ejector refrigeration using lumped parameter models," International Journal of Refrigeration, vol. 58, pp. 154-171, 2015. 
[17] Boumaraf Latra and Haberschill Philippe, "Investigation of a novel ejector expansion refrigeration system using the working fluid R134a and its potential substitute R1234yf," Energy Economics, vol. 45, pp. 148-159, 2014.

[18] Lin Chen, Li Yanzhong, Cai Wenjian, Yan Jia and $\mathrm{Hu} \mathrm{Yu,} \mathrm{"Experimental}$ investigation of the adjustable ejector in a multi-evaporator refrigeration system," Applied Thermal Engineering, vol. 61, pp. 2-10, 2013.

[19] Xing Meibo, Yan Gang and Yu Jianlin, "Performance evaluation of an ejector subcooled vapor-compression refrigeration cyclr," Energy Conversion and Management, vol. 92, pp. 431-436, 2015.

[20] Huang BJ, Wu JH, Hsu HY and Wang JH, "Development of hybrid solar-assisted cooling/heating system," Energy Conversion and Management, vol. 51, pp. 16431650, 2010.

[21] Huang BJ, Petrenko VA, Chang JM, Lin CP and Hu SS, "A combined-cycle refrigeration system using ejector cooling cycle as the bottom cycle," International Journal of Refrigeration, vol. 24, pp. 391-399, 2001.

[22] Ersoy HK and Billir Sag N, "Preliminary experimental result on the R134a refrigeration system using a two-phase ejector as an expander," International Journal of Refrigeration, vol. 43, pp. 97-110, 2014.

[23] National Environment Agency (Singapore), "Weather Statistics," Retrieved 25 April 2016.

[24] W. F. Stoecker, Industrial refrigeration handbook. New York, USA: McGraw-Hill, 1998.

[25] Nowakowski R. S, "Stable neuron numbers from cradle to grave," Proceedings of the National Academy of Sciences, vol. 33, pp. 12219-12220, 2006.

[26] S. S. Haykin, Neural networks and learning machines, $3^{\text {rd }}$ edition. New York: Prentice Hall, 2009.

[27] Martin Anthony, Discrete Mathematics of Neural Networks: Selected Topics, SIAM, 2001.

[28] Charu C. Aggarwal, Data Classification: Algorithms and Applications. CRC Press, 2014.

[29] S. S. Haykin, Neural networks and learning machines, $3^{\text {rd }}$ edtion. New York: Prentice Hall, 2009.

[30] Paul Werbos, "Beyond Regression: New Tools for Prediction and Analysis in the Behavioral Sciences," PhD thesis, Harvard University, 1974.

[31] Paul Werbos, "Backpropagation through time: what it does and how to do it," Proceedings of the IEEE, vol.78, pp. 1550-1560, 1990.

[32] Broomhead D.S and Lowe David, "Radial basis functions, multi-variable functional interpolation and adaptive networks, " RSRE, 1988.

[33] Broomhead D.S and Lowe David, "Multivariable functional interpolation and adaptive networks," Complex Systems, vol. 2, pp. 321-355, 1988.

[34] Schwenker Friedhelm, Kestler Hans A and Palm Guither, "Three learning phases for radial-basis-function networks," Neural Networks, vol. 14, pp. 439-458, 2001.

[35] Park J and I. W. Sandberg, "Universal Approximation Using Radial-basis-function Networks," Neural Computation, vol. 3, pp. 246-257, 1991.

[36] T. Poggio and F. Girosi, "Networks for approximation and learning," Proceedings of IEEE, vol. 78, pp. 1484-1487, 1990. 
[37] Yee Paul V and Haykin Simon, Regularized radial basis function networks: Theory and Applications. John Wiley, 2001.

[38] V. N. Vapnik, Statistical learning theory. New York: Wiley, 1998.

[39] V. N. Vapnik, The nature of statistical learning theory, $2^{\text {nd }}$ edition. New York: Springer, 2000.

[40] Ben Hur, Asa, Horn, David, Siegelmann, Hava and Vapnik, "Support vector clustering," Journal of Machine Learning Research, vol. 2, pp. 125-137, 2001.

[41] Meyer D, Leisch F and Hornik K, "The support vector machine under test," Neurocomputing, vol. 55, pp. 169, 2003.

[42] B. H. Boyle, Support vector machines: data analysis, machine learning and applications. New York: Nova Science Publishers, 2011.

[43] W. F. Schmidt, M. A. Kraaijveld and R. P. W. Duin, "Feedforward neural networks with random weights," in Proc. of $11^{\text {th }}$ IAPR International Conference on Pattern Recognition, Conference B: Pattern Recognition Methodology and Systems, vol. 2. IEEE, pp. 1-4, 1992.

[44] Huang G. B, Zhu Q. Y and Siew C. K, "Extreme learning machine: a new learning scheme of feedforward neural networks," in 2004 IEEE International Joint Conference on Neural Networks, 25-29 July 2004, Piscataway, NJ, USA, 2004, pp. 985-990.

[45] Huang G. B, Zhu Q. Y and Siew C. K, "Extreme learning machine: theory and applications," Neurocomputing, vol. 70, pp. 489-501, 2006.

[46] Huang G. B and Zhou H. M, "Extreme Learning Machine for Regression and Multiclass Classification," IEEE Transcations on systems, man, and cybernetics. vol. 42, pp. 513-529, 2012.

[47] Huang G. B, "What are Extreme Learning Machines? Filling the Gap Between Frank Rosenblatt's Dream and John Von Neumann's Puzzle," Cognitive Computing, vol. 7, pp. 263-278, 2015.

[48] Breiman L, "Bagging predictors," Machine Learning, vol. 26, pp. 123-140, 1996.

[49] Freund Y and Schapire R, "Experiments with a new boosting algorithm," In Machine Learning: Proceedings of the Thirteenth International Conference, 148156, 1996.

[50] Friedman J. H, Greedy Function Approximation: a Gradient Boosting Machine. Technical report, Dept. of Statistics, Stanford University, 1999.

[51] C. A. Hooker, Adaptation in Natural and Aritifical Systems- Holland, Jh.

[52] D. E. Goldberg, Genetic algorithms in search, optimization, and machine learning. Reading, Mass, USA: Addison-Wesley Pub.Co., 1989.

[53] Akbari Ziarati, "A multilevel evolutionary algorithm for optimizing numerical functions," IJIEC, vol. 2, pp. 419-430, 2011.

[54] D. E. Goldberg, The design of innovation: lessons from and for competent genetic algorithms. Boston: Kluwer Academic Publishers, 2002.

[55] R. B. Hollstien, "Artificial genetic adaptation in computer control systems," University Microfilms, Ann Arbor, MI, 1971.

[56] Fraser Alex, "Simulation of genetic systems by automatic digital computers. 1. Introduction," Aust. J. Biol. Sci, vol. 10, pp. 484-491, 1957. 
[57] Rania Hassan, Babak Cohanim and Olivier de Weck, "A comparison of particle swarm optimization and the genetic algorithm," American Institute of Aeronautics and Astronautics, 2005.

[58] Kennedy J and Eberhart R, "Particle Swarm Optimization," Proceedings of IEEE International Conference on Neural Networks, vol. 10, pp. 1942-1948, 1995.

[59] Shi Y and Eberhart R, "A modified particle swarm optimizer," Proceedings of IEEE International Conference on Evolutionary Computation, pp. 69-73, 1998.

[60] Boon Chiang Ng, Daris, I.Z.M and Kamar, H.M, "Application of multilayer perceptron and radial basis function neural network in steady state modeling of automotive air conditioning system," in 2012 IEEE International Conference on Control System, 2012.

[61] Dehestani, D and Su, S, "Robust fault tolerant application for HVAC system based on combination of online SVM and ANN black box model," in 2013 European Control Conference, July 17- 19, 2013.

[62] Md Lazin, M.N, Mat and Darus, I.Z, "Genetic algorithm identification for automotive air-conditioning system," in 2013 IEEE Symposium on Computers \& Informatics, April 7-9, 2013.

[63] Md Lazin, M.N, Mat and Darus, I.Z, "Identification for automotive airconditioning system using Particle Swarm Optimization," in 2013 Australian Control Conference, Nov 4-5, 2013.

[64] Y.V.C. Rao, An Introduction to Thermodynamics, $2^{\text {nd }}$ ed. Universities Press, 2003.

[65] R. J. Dossat and T. J. Horan, Principles of refrigeration, $5^{\text {th }}$ ed. Upper Saddle River, N.J., U.S.A: Prentice Hall, 2002.

[66] Smith. F. E, "Sir Charles Parsons and steam," Mechanical World, vol. 100, pp. 495-497, 1936.

[67] Anon, "The Leblanc ejector condenser," Engineering, 1909.

[68] Besagni and Giorgio, "Ejector refrigeration: A comprehensive review," Renewable and Sustainable Energy Reviews, vol. 53, pp. 373-407, 2016.

[69] Galanis and Nicolas, "Ejector design and performance prediction," International Journal of Thermal Sciences, vol. 104, pp. 315-329, 2016.

[70] Sokolov M and Hershgal D, "Enhanced ejector refrigeration cycles powered by low-grade heat," International Journal of Refrigeration, vol. 13, pp. 351-356. 1990.

[71] Dorantes R and Estrada CA, "Mathematical simulation of a solar ejectorcompression refrigeration system," Applied Thermal Engineering, vol. 16, pp. 669-675, 1996.

[72] Sarkar J, "Ejector enhanced vapor compression refrigeration and heat pump systems-a review," Renew Sustain Energy, vol. 19, pp. 6647-6659, 2012.

[73] Kornhauser AA and Menegay P, "Method of reducing flow metastability in an ejector nozzle," Office USPaT, Washington DC, 1994.

[74] Nakagawa $M$ and Takeuchi $H$, "Performance of two phase ejector in refrigeration cycle," Transaction Japan Soc Mech Engineer, vol. 64, pp. 1-8, 1998.

[75] NI Instrument Driver Network, "http://www.ni.com/downloads/instrumentdrivers/."

[76] Reinhard Radermacher and Ling Jiazhen, "Thoughts on refrigerant choices," HVAC\&R Research, vol. 18, pp. 295-296. 2012. 
[77] Wang Hao, Cai Wenjian, Wang Youyi, Yan Jia and Wang Lei, "Experimental study of the behavior of a hybrid ejector-based air-conditioning system with R134a," Energy Conversion and Management, vol. 112, pp. 31-40, 2016.

[78] Thomas L. Mckinley and Andrew G. Alleyne, "An advanced nonlinear switched heat exchanger model for vapor compression cycles using the moving-boundary method," International Journal of Refrigeration, vol. 31, pp. 1253-1264, 2008.

[79] T.S. Ge, Y.J. Dai and R.Z. Wang, "Performance study of silica gel coated fin-tube heat exchanger cooling system based on a developed mathematical model," Energy Conversion and Management, vol. 52, pp. 2329-2338, 2011.

[80] Liu Jian, Wei Wenjian and Ding Gouliang, "A general steady state mathematical model for fin-and-tube heat exchanger based on graph theory," International Journal of Refrigeration, vol. 27, pp. 965-973, 2004.

[81] Yasar Islamoglu, "A new approach for the prediction of the heat transfer rate of the wire-on-tube type heat exchanger-use of an artificial neural network model," Applied Thermal Engineering, vol. 23, pp. 243-249, 2003.

[82] C.K. Tan and J. Ward, "Artificial neural network modelling of the thermal performance of a compact heat exchanger," Applied Thermal Engineering, vol. 29, pp. 3609-3617, 2009.

[83] Gerardo Diaz and Mihir Sen, "Dynamic prediction and control of heat exchangers using artificial neural networks," International Journal of Heat and Mass Transfer, vol. 44, pp. 1671-1679, 2001.

[84] Ismael R. Maestre and F. Javier Gonzalez Gallero, "Performance assessment of a simplified hybrid model for a vertical ground heat exchanger," Energy and Buildings, vol. 66, pp. 437-444, 2013.

[85] Zhao Lei, "A modified hybrid model of heat exchanger based on fouling effect," in $20096^{\text {th }}$ IEEE Conference on Industrial Electronics and Applications, ICIEA 2009.

[86] Ding Xudong, Cai Wenjian and L. Jia, "Evapoartor modeling- A hybrid approach," Applied Energy, vol. 86, pp. 81-88, 2009.

[87] Ding Xudong, Cai Wenjian and L. Jia, "A hybrid condenser model for real-time applications in performance monitoring, control and optimization," Energy Conversion and Management, vol. 50, pp. 1513-1521, 2009.

[88] Ding Xudong, Cai Wenjian and L. Jia, "A hybrid modeling for the real-time control and optimization of compressors," in $20094^{\text {th }}$ IEEE Conference on Industrial Electronics and Applications, ICIEA 2009, May 25, 2009 - May 27, 2009, Xi'an, China, 2009, pp. 3256-3261.

[89] Sarkar Jahar, "Ejector enhaced vapor compression refrigeration and heat pump systems- A review," Renewable and Sustainable Energy Reviews, vol. 16, pp. 6647-6659, 2012.

[90] Zhu Yinhai, Cai Wenjian and Wen Changyun, "Shock circle model for ejector performance evaluation," Energy Conversion and Management, vol. 48, pp. 25332541, 2007.

[91] Syed M. Zubair and Anwar K. Sheikh, "A risk based heat exchanger analysis subject to fouling Part 1: Performance evaluation," Energy, vol. 25, pp. 427-443, 2000 . 
[92] Kern DQ and Seaton RE, "A theoretical analysis of thermal surface fouling," $\mathrm{Br}$ Chemistry Engineering, vol. 5, pp. 258-262, 1959.

[93] Taborek J and Aoki T, "Fouling: the major unresolved problem in heat transfer," Chemistry Engineering Program, vol. 68, pp. 59-67, 1972.

[94] Khan MS and Zubair SM, "Fouling resistance model for prediction of $\mathrm{CaCO}_{3}$ scaling in AISI 316 tubes," Heat Mass Transfer, vol. 32, pp. 73-80, 1996.

[95] Muller-Steinhagen H and Reif F, "Influence of operating conditions on particulate fouling," Can J Chemistry Engineering, vol. 66, pp. 42-50, 1988.

[96] Hasson D, Progress in precipitation fouling research-a review. New York: Begell House, 1999.

[97] Tian Z and Qian Ch, "Electric vehicle air conditioning system performance prediction based on artificial neural network," Applied Thermal Engineering, vol. 89, pp. 101-114, 2015.

[98] Jani DB and Mishra Manish, "Performance prediction of rotary solid desiccant dehumidifier in hybrid air-conditioning system using artificial neural network," Applied Thermal Engineering, vol. 98, pp. 1091-1103, 2016.

[99] Li Zhao and Xu Xiangguo, "A novel neural network aided fuzzy logic controller for a variable speed (VS) direct expansion (DX) air-conditioning (A/C) system," Applied Thermal Engineering, vol. 78, pp. 9-23, 2015.

[100] Curtiss PS and Kreider JF, "Experimental results of a predictive neural network HVAC controller," ASHRAE Trans, pp. 192-197, 1998.

[101] Yilmaz S and Atik K, "Modeling of a mechanical cooling system with variable cooling capacity by using artificial neural network," Applied Thermal Engineering, vol. 27, pp. 2308-2313, 2007.

[102] Massie and Darrell D, "Neural network optimal controller for commercial ice thermal storage systems," ASHRAE Transcations, vol. 110, pp. 361-369, 2004.

[103] Huang Guangbing, D. H. Wang and Y. Lan, "Extreme learning machines: a survey," International Journal of Machine Learning and Cybernetics, vol. 2, pp. 107-122, 2011.

[104] Fu Heming and Vong Chi-man, "Fast detection of impact location using kernel extreme learning machine," Neural Computing and Applications, vol. 27, pp. 121130, 2016.

[105] Li Xiaodong and Xie Haoren, "Empirical analysis: stock market prediction via extreme learning machine," Neural Computing and Applications, vol. 27, pp. 6778, 2016.

[106] Qin Longhui and Hu Liang, "Application of extreme learning machine to gas flow measurement with multipath acoustic transducers," Flow Measurement and Instrumentation, vol. 49, pp. 31-39, 2016.

[107] Pak Kin Wong and Hang Cheong Wong, "Model predictive engine air-ratio control using online sequential extreme learning machine," Neural Computing and Applications, vol. 27, pp. 79-92, 2016.

[108] J. Hammon, Optimisation combinatorie pour la selectionde variables en regression en grande dimension: Application en genetique animale. 2013.

[109] Gareth James, An Introduction to Statistical Learning. Springer press, 2013. 
[110] Wang Hao, Cai Wenjian and Wang Youyi, "Modeling of a Hybrid Ejector Air Conditioning System using Artificial Neural Networks," Energy Conversion and Management, vol. 127, pp. 11-24, 2016.

[111] S. Sanaye, "Thermal and economical optimization of air conditioning units with vapor compression refrigeration systems," Applied Thermal Engineering, vol. 24, pp. 1807-1825, 2004.

[112] Jameel-ur-Rehman Khan and Syed M. Zubair, "Thermodynamic optimization of finite time vapor compression refrigeration systems," Energy Conversion and Management, vol. 42, pp. 1457-1475, 2001.

[113] Goktun Selahattin, "Optimization of a solar-driven irreversible Carnot heat engine at maximum power output," Energy Sources, vol. 19, pp. 661-664, 1997.

[114] Yuwen You, Aiguo Wu and Shu Yang, "An optimal control strategy for multievaporator vapor compression systems," International Conference on Electrical and Control Engineering, 2010.

[115] Daniel J. Burns, Walter K. Weiss and Martin Guay, "Realtime Setpoint Optimization with time-varying Extremum Seeking for vapor compression systems," American Control Conference, 2015.

[116] Neera Jain and Andrew Alleyne, "Exergy-based optimal control of a vapor compression system," Energy Conversion and Management, vol. 92, pp. 353-365, 2015.

[117] Velimir Congradac and Filip Kulic, "HVAC system optimization with $\mathrm{CO}_{2}$ concentration control using genetic algorithms," Energy and Buildings, vol. 41, pp. 571-577, 2009.

[118] W. Huang and H. N. Lam, "Using genetic algorithms to optimize controller parameters for HVAC systems," Energy and Buildings, vol. 26, pp. 277-282, 1997.

[119] Lu Lu and Cai Wenjian, "Global optimization for overall HVAC systems- Part 2 problem solution and simulations," Energy Conversion and Management, vol. 46, pp. 1015-1028, 2005.

[120] Janghoo Seo, Ryozo Ooka, Jeong Tai Kim and Yujin Nam, "Optimization of HVAC system design to minimize primary energy demand," Energy and Building, vol. 76, pp. 102-108, 2014.

[121] Beghi Alessandro, "A PSO-based algorithm for optimal multiple chiller systems operation," Applied Thermal Engineering, vol. 32, pp. 31-40, 2012.

[122] He Xiaofei, Zhang Zijun and Andrew Kusiak, "Performance optimization of HVAC systems with computational intelligence algorithms," Energy and Buildings, vol. 81, pp. 371-380, 2014.

[123] Y. H. Pao, G. H. Park and D. J. Sobajic, "Learning and generalization characteristics of random vector functional-link net," Neurocomputing, vol. 6, pp. 163-180, 1994.

[124] D. Lowe, "Adaptive radial basis function nonlinearities, and the problem of generalisation," Proc. $1^{\text {st }}$ Inst. Electrical Engineering International Conference Artificial Nueral Network, pp. 171-175, 1989.

[125] G. Inalhan, "Decentralized Optimization across independent decision makers with incomplete mdoels," Ph.D. 3145523, Stanford University, United States California, 2004. 
[126] Wang Shiqiang, "Decentralized Optimization for a Novel Control Structure of HVAC System," Mathematical Problems in Engineering, vol. 13, pp. 2538-2551, 2016.

[127] Al-Assadi. S.A.K, "Robust decentralized control of HVAC systems using H $\infty-$ performance measures," Journal of the Franklin Institutue, vol. 341, pp. 543-567, 2004.

[128] Dai Yunchuang, "A decentralized algorithm for optimal distribution in HVAC systems," Building and Environment, vol. 95, pp. 21-31, 2016.

[129] Hemidi Amel, "CFD analysis of a supersonic air ejector. Part 1: Experimental validation of single-phase and two-phase operation," Applied Thermal Engineering, vol. 29, pp. 1523-1531, 2009.

[130] Zhang Qi, "Numerical simulation and performance study of adjustable steam ejector using CFD," Advanced Materials Research, vol. 562-564, pp. 1150-1154, 2012.

[131] Petrenko V.O, "Design theoretical study of cascade $\mathrm{CO}_{2}$ sub-critical mechanical compression/butane ejector cooling cycle," International Journal of Refrigeration, vol. 34, pp. 1649-1656, 2011.

[132] Gaafar Abdella Mohammed. Y, "Exergy analysis of a solar-driven dual parallelconnected ejector refrigeration system," Applied Mechanics and Materials, vol. 839, pp. 100-106, 2016.

[133] Hai Gang Zhang, "A novel improved ELM algorithm for a real industrial application, " Mathematical Problems in Engineering, vol. 82, pp. 765-772, 2014.

[134] Kusiak. A, "Multi-objective optimization of HVAC system with an evolutionary computation algorithm," Energy, vol. 36, pp. 2440-2449, 2011.

[135] Wei Xiupeng, "Multi-objective optimization of the HVAC (heating, ventilation, and air conditioning) system performance," Energy, vol. 83, pp. 294-306, 2015.

[136] Hamdy. M, "Optimum design of a house and its HVAC systems using simulationbased optimisation," International Journal of Low Carbon Technologies, vol. 5, pp. 120-124, 2010. 